\title{
Neuaufbau eines \\ Pelletron-Beschleunigers \\ und Untersuchungen zum Laserhydrieren von Silizium
}

\author{
Dissertation \\ zur Erlangung des Doktorgrades \\ der Mathematisch-Naturwissenschaftlichen Fakultäten \\ der Georg-August-Universität zu Göttingen
}

vorgelegt von

Marcus Schwickert

aus Koblenz

Göttingen 2002 
D7

Referent: Prof. Dr. K. P. Lieb

Korreferent: Prof. Dr. H.-U. Krebs

Tag der mündlichen Prüfung: 29.10.2002 


\section{Meinem Vater Werner Schwickert, der alles reparieren konnte, außer seiner Gesundheit.}





\section{Inhaltsverzeichnis}

\begin{tabular}{|lll}
\hline 1 & Einleitung & 3
\end{tabular}

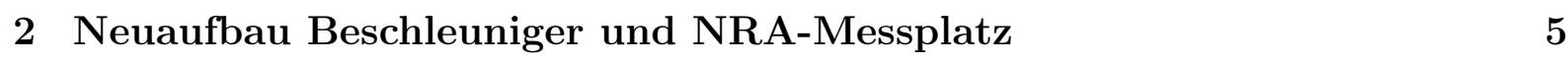

2.1 Umzug und Neuinstallation . . . . . . . . . . . . . . . . . . 6

2.1 .1 Ionenquellen . . . . . . . . . . . . . . . . . . . 10

2.1 .2 Beschleuniger-Tank . . . . . . . . . . . . . . . . . . . . . 12

$2.1 .3 \quad$ Energiestabilisierung . . . . . . . . . . . . . . . . . . 13

2.2 Resonante Kernreaktionsanalyse (NRA) . . . . . . . . . . . . . . . . . . . 15

2.2 .1 Beschreibung der Methode . . . . . . . . . . . . . . 15

$2.2 .2 \quad 0^{\circ}$-Strahlrohr und Low-Level Messaufbau . . . . . . . . . . . . . . . 19

$2.3 \quad$ Energieeichung von MaRPel . . . . . . . . . . . . . . . . . . . . . . . . . . 22

$2.3 .1 \quad$ Energiebreite des Ionenstrahls . . . . . . . . . . . . . . . . . . 22

2.3 .2 Energieeichung des $90^{\circ}$-Magneten . . . . . . . . . . . . . 26

2.3 .3 Amsel-Energie-Scanning-System . . . . . . . . . . . . . . . . . . . . 29

2.3 .4 Messdatenerfassung mit KNRA . . . . . . . . . . . . . . . . 33

2.3 .5 Empfindlichkeit und Reproduzierbarkeit . . . . . . . . . . . . . . 35

\begin{tabular}{lll}
\hline 3 & Analysemethoden & 37
\end{tabular}

$3.1 \quad$ Rutherford-Rückstreuspektrometrie (RBS) . . . . . . . . . . . . . . . . . 37

3.1 .1 RBS-Tiefenprofile . . . . . . . . . . . . . . . . . . . . . 38

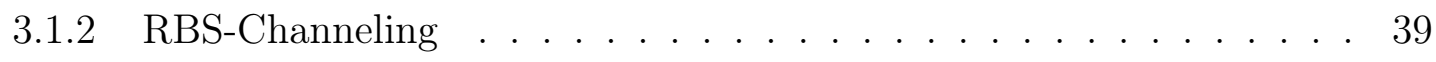

3.2 Oberflächenprofilometrie . . . . . . . . . . . . . . . . . . . 41

4 Grundlagen zu H-Implantation und Laserhydrieren 43

$4.1 \quad$ Defekterzeugung und Amorphisierung durch Ionenstrahlen . . . . . . . . . 43

4.2 Grundlagen des Laserhydrierens . . . . . . . . . . . . . . . . . . . . . . . . 46

4.3 Laserinduzierte Rekristallisation von Silizium . . . . . . . . . . . . . . . . . 52

4.4 Wasserstoff-Diffusion . . . . . . . . . . . . . . . . . . . . . . . 54 
$\begin{array}{lll}5 & \text { Probenpräparation } & 56\end{array}$

5.1 Excimer-Laser . . . . . . . . . . . . . . . . . . . . 56

5.2 Ionenimplantation . . . . . . . . . . . . . . . . . 57

5.3 Probenübersicht . . . . . . . . . . . . . . . . . . . . . . . . . . . . . . . 58

$\begin{array}{lll}6 & \text { Ergebnisse und Diskussion } & 59\end{array}$

6.1 Laserhydrieren von Titan . . . . . . . . . . . . . . . . . . . . . . . . . . . . 59

6.2 Laserhydrieren von kristallinem Silizium . . . . . . . . . . . . . . . . . 65

6.3 Laserhydrieren von amorphisiertem Silizium . . . . . . . . . . . . . . . . 70

$6.3 .1 \quad$ Laserhydrieren von Xe-implantiertem Silizium . . . . . . . . . . . . 70

6.3 .2 Laserhydrieren von H-implantiertem Silizium. . . . . . . . . . . . . 81

\begin{tabular}{lll}
\hline 7 & Zusammenfassung und Ausblick & 90
\end{tabular}

7.1 Laserhydrieren von Titan . . . . . . . . . . . . . . . . . . . . . . . . . . . . 90

7.2 Laserhydrieren von Silizium . . . . . . . . . . . . . . . . . . . . . 91

\begin{tabular}{ll}
\hline Literaturverzeichnis & 97
\end{tabular}

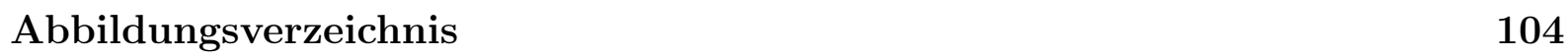

\begin{tabular}{ll}
\hline Anhang & 106
\end{tabular}

\begin{tabular}{|l|l|}
\hline A Relativistische Rechnung zum 90'-Magneten & 107
\end{tabular}

\begin{tabular}{ll}
\hline B Amsel-Steering-System & 109
\end{tabular}

B.1 Amselgeometrie . . . . . . . . . . . . . . . . . . . . . 109

B.2 Exakte Berechnung des Verstärkungsfaktors G(E) . . . . . . . . . . . . . . 110

B.3 Näherung für den Verstärkungsfaktor G(E) . . . . . . . . . . . . . . . . . . 111

B.4 Numerische Bestimmung von $\mathrm{G}(\mathrm{E})$. . . . . . . . . . . . . . . . . . . . . . 112

B.5 Geometrische Begrenzung der Deflektor-Spannung . . . . . . . . . . . . . . 113

B.6 $\quad$ Korrektur zur Nichtlinearität von $\mathrm{G}(\mathrm{E})$. . . . . . . . . . . . . . . . . . . . 114

B.7 Relativistische Berechnung von $\mathrm{G}(\mathrm{E})$. . . . . . . . . . . . . . . . . . . 115

\begin{tabular}{|l|l}
\hline C Nachweisempfindlichkeit der Low-Level NRA & 117 \\
\hline
\end{tabular} 


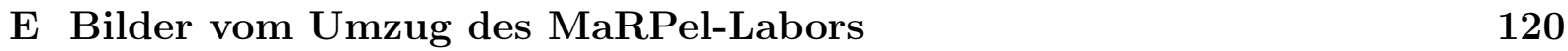

E.1 Abbau in Heidelberg . . . . . . . . . . . . . . . . . . . . . . . . . . . . 120

E.2 Aufbau in Göttingen . . . . . . . . . . . . . . . . . . . . . . . . 122

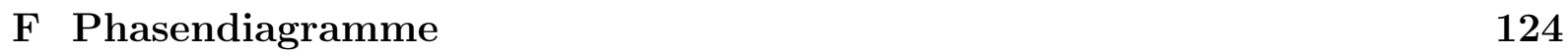

F.1 Ti-H-Phasendiagramm . . . . . . . . . . . . . . . . . . . . . 124

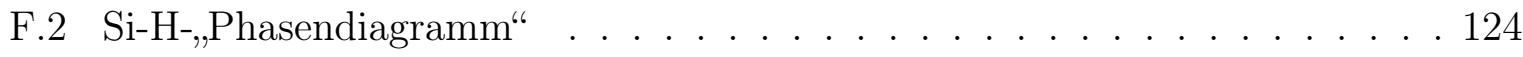

\begin{tabular}{lr}
\hline Lebenslauf & 127
\end{tabular}

\begin{tabular}{lr}
\hline Danksagung & 127
\end{tabular} 



\section{Zusammenfassung}

Im Rahmen dieser Arbeit wurde eine Pelletron-Beschleunigeranlage NEC 9SDH-2 vom Max-Planck-Institut für Kernphysik Heidelberg zum II. Physikalischen Institut der Universität Göttingen transferiert und hier neu aufgebaut. Zu dieser Anlage gehören ein sogenanntes „Amsel-Deflektor"-System und ein Low-Level Messplatz für die resonante Kernreaktionsanalyse, deren Kombination die experimentellen Voraussetzungen zum hochgenauen Wasserstoffnachweis bietet. Diese Methode wurde in der vorliegenden Arbeit dazu benutzt, die Wasserstoffaufnahme von Titan- und Siliziumproben beim "Laserhydrieren“ zu messen. Mit „Laserhydrieren“ wird die Laserbestrahlung von Materialien in Wasserstoffatmosphäre bezeichnet, die nach unserem Wissen in dieser Arbeit zum ersten Mal durchgeführt und genauer untersucht wurde.

Beim Neuaufbau des Pelletrons in Göttingen wurden folgende Elemente verbessert bzw. neu konzipiert:

1. Die Alphatross-Ionenquelle wurde mit einer neuen Gasregulierung versehen, so dass nun ein Strahlstrom von $\mathrm{I}_{\mathrm{He}} \approx 400 \mathrm{nA}$ für He-Ionen erreicht wird. Die FeststoffIonenquelle (SNICS) liefert durch den Umbau des Kühlsystems und die Verwendung eigens angefertigter Sputtertargets Strahlströme von $\mathrm{I}_{P} \approx 30 \mu \mathrm{A}$ für Protonen und $\mathrm{I}_{N} \approx 20 \mu \mathrm{A}$ für den ${ }^{15} \mathrm{~N}$-Strahl.

2. Vor der Inbetriebnahme musste der Hochdruck-Beschleunigertank mehrmals komplett zerlegt und gereinigt werden. Das darin enthaltene Ladungssystem zur Erzeugung der $3 \mathrm{MV}$-Hochspannung wurde repariert und bezüglich der Hochspannungsfestigkeit verbessert.

3. Durch den Umbau und die Neujustierung des $90^{\circ}$-Massenseparators der Anlage wurde eine Energie-Eichung des Beschleunigers nötig. Die Messung mehrerer ${ }^{15} \mathrm{~N}\left({ }^{1} \mathrm{H}, \alpha \gamma\right){ }^{12} \mathrm{C}-$ Kernresonanzen erlaubte die Bestimmung des Krümmungsradius des $90^{\circ}$-Massenseparators zu $\mathrm{R}=0.871(1) \mathrm{m}$.

4. Da bei den ersten Testläufen die Energie des Ionenstrahls sehr stark schwankte, wurden alle Teile der Regelelektronik zur Energiestabilisierung des Beschleunigers mehrfach auf Fehler getestet und zum Teil ausgetauscht. Eine neu entworfene Regeleinheit zur Steuerung und Anzeige aller internen Fehlersignale des Beschleunigers erlaubt nun eine sehr hohe Stabilität der Strahlenergie. Für einen $1 \mathrm{MeV}$-Protonenstrahl wurde experimentell eine Energieaufweitung von nur $\Delta \mathrm{E}=0.48(3) \mathrm{keV}$ gemessen.

5. Ein System von „Amsel-Deflektor"-Platten zur hysteresefreien Energieverstellung des Beschleunigers wurde aufgebaut, justiert und mit einer neuen Computersteuerung versehen. Damit ist es nun möglich, die Strahlenergie in einem großen Bereich um die Resonanzenergie in sehr kleiner Schrittweite zu variieren. Der Verstärkungsfaktor $\mathrm{G}_{0}$, der die grundlegende Größe des „Amsel-Systems“ ist, wurde jeweils experimentell für den ${ }^{15} \mathrm{~N}$ - und Protonenstrahl ermittelt und mit dem Theoriewert verglichen. Experimentell ergab sich für den ${ }^{15} \mathrm{~N}-\mathrm{Strahl} \mathrm{G}_{0}\left({ }^{15} \mathrm{~N}\right)=7.97(8)$ und für den Protonenstrahl $\mathrm{G}_{0}(\mathrm{p})=5.15(5)$. Abweichungen vom linearen Verhalten der Deflektor-Platten wurden für verschiedene Ionensorten, auch im relativistischen Grenzfall, berechnet. 
6. Nach dem Aufbau und der Inbetriebnahme des Low-Level Messplatzes für die resonante Kernreaktionsanalyse wurde das Strahlrohr mit einer Zweifach-ViersektorenBlende zur genauen Kontrolle der Position des Analysestrahls versehen und die computergestützte Messdatenerfassung neu konzipiert. Zur Aufnahme von Gammaspektren wurde ein Echtzeit-Computerprogramm entwickelt, welches zusätzlich eine vollautomatisierte Messdatenaufnahme und Variation der Energie des Beschleunigers ermöglicht. Das Programm erlaubt ebenfalls eine Untergrundkorrektur und die Aufbereitung der anfallenden Messdaten während der laufenden Messung. Die Kammerkonstante des Low-Level Messplatzes wurde $\mathrm{zu} \mathrm{K}=21.126$ cts $/(\mu \mathrm{C}$ at $\% \mathrm{H}$ in $\mathrm{Si})$ bestimmt, woraus sich eine minimal nachweisbare Wasserstoff-Konzentration von $\mathrm{c}_{\min }(\mathrm{H})=450$ atppm ergibt.

Mit Hilfe dieses Messplatzes zur resonanten Kernreaktionsanalyse wurde die Wasserstoff-Aufnahme von laserhydrierten Titan- und Siliziumproben untersucht. Das Laserhydrieren von Titan führt zu einem erhöhten Wasserstoffgehalt von bis zu 47 at\% in der experimentell zugänglichen Tiefe von $370 \mathrm{~nm}$. Wie XRD-Messungen belegen, kommt es beim Laserhydrieren des Titans zur Bildung von $\mathrm{TiH}_{2}$.

Messungen zum Laserhydrieren von kristallinem und ionenstrahl-amorphisiertem Silizium zeigen nur eine geringe Wasserstoffaufnahme durch das Laserhydrieren. Im Falle des kristallinen Siliziums liegt der maximale Wasserstoffgehalt nach dem Laserhydrieren bei ca. 8 at\% nahe der Probenoberfläche und sinkt in einer Tiefe von $70 \mathrm{~nm}$ unter 1 at\%. RBSChanneling Messungen dieser Proben zeigen eine starke Schädigung im oberflächennahen Bereich, die als Diffusionsfalle für den beim Laserhydrieren eingebrachten Wasserstoff wirkt.

Das Laserhydrieren von Xe-amorphisierten Siliziumproben führt bei Laserfluenzen von $\mathrm{F} \leq 1.5 \mathrm{~J} / \mathrm{cm}^{2}$ zur Bildung von polykristallinem Silizium mit einer mittleren Kristallitgröße von 64(8) nm, bei einem sehr geringen Wasserstoff-Eintrag. Wird die Laserfluenz erhöht, so zeigen RBS-Channeling Messungen die epitaktische Rekristallisation der amorphen Deckschicht.

Das Laserhydrieren von Siliziumproben, in denen durch H-Implantationen eine vergrabene amorphe Schicht erzeugt worden war, führt dagegen schon bei kleinen Laserfluenzen $\mathrm{F} \leq 1 \mathrm{~J} / \mathrm{cm}^{2}$ zu einer Zunahme der Schädigung und einer Abnahme des WasserstoffGehalts. 


\section{Einleitung}

Seit den 60er Jahren des 20. Jahrhunderts haben sich Ionenstrahlen sowohl zu einem Standardwerkzeug zur Materialmodifikation, als auch zur Analyse der atomaren Zusammensetzung von Materialien aller Art entwickelt [1]. Durch Ionenimplantation bei einigen hundert $\mathrm{keV}$ bis $\mathrm{MeV}$ lassen sich bis in eine Tiefe von einigen hundert Nanometern Schichten in beinahe beliebiger Zusammensetzung erzeugen. Die Methoden der Ionenstrahlanalytik bieten passend dazu die Möglichkeit, schon kleinste Mengen von Fremdatomen isotopenspezifisch und häufig zerstörungsfrei nachzuweisen.

Im Zuge der vorliegenden Arbeit wurden eine Beschleunigeranlage und ein Messplatz für Kernreaktionsanalyse im II. Physikalischen Institut der Universität Göttingen neu installiert. Diese Beschleunigeranlage erweitert die Möglichkeiten der in Göttingen bereits etablierten Methoden zur Ionenstrahlanalyse und ionenstrahl-gestützten Materialmodifikation enorm. Mit dem Göttinger Schwerionenimplantator IONAS [2] wurde bisher eine Maximalenergie von $1 \mathrm{MeV}$ für doppelt geladene Ionen erreicht. Mit dem neu installierten Labor wird der maximal zugängliche Energiebereich um eine Größenordnung auf $9 \mathrm{MeV}$ erweitert. Diese Erweiterung eröffnet die Möglichkeit, Protonen-Kernresonanzen

aller leichten Elemente zur Elementanalyse zu nutzen, denn die wichtigsten Resonanzen liegen im bislang nicht zugänglichen Bereich zwischen 1 und $3 \mathrm{MeV}$.

Auch für die Rückstreuspektroskopie stellt die Verfügbarkeit von Heliumstrahlen mit Energien von bis zu $6 \mathrm{MeV}$ einen großen Vorteil dar, denn oft lassen sich Fragestellungen der Dünnschichtanalyse erst durch die Verwendung unterschiedlicher Projektilenergien lösen. Insbesondere die neu geschaffene Möglichkeit, Sauerstoff mittels resonanter Rückstreuung von Alphateilchen nachweisen zu können, eröffnet ein breites Spektrum für zukünftige Anwendungen der Materialforschung. Die Auslegung der Rückstreu-Kammer mit einem Dreiachsen-Goniometer und einem Multidetektor-System liefert die besten Voraussetzungen für die hochpräzise Elementanalyse mit guter Statistik bei kurzen Messzeiten.

Das Hauptinstrument dieser Arbeit ist jedoch der Low-Level Messplatz zur resonanten Kernreaktionsanalyse („nuclear reaction analysis“, NRA). Der Aufbau zeichnet sich besonders durch eine hohe Nachweisempfindlichkeit aus, die durch eine Kombination aus aktiven und passiven Abschirmmaßnahmen erreicht wird. In Verbindung mit dem System zur exakten Variation der Beschleuniger-Energie (,Amsel-Deflektoren“) können NRA-Messungen stark automatisiert durchgeführt werden. Die resonante Kernreaktionsanalyse wird in dieser Arbeit zur Bestimmung von Wasserstofftiefenprofilen laserbehandelter Silizium- und Titanproben verwendet. Die hierzu benutzte Kernresonanz ${ }^{1} \mathrm{H}\left({ }^{15} \mathrm{~N}, \alpha \gamma\right){ }^{12} \mathrm{C}$ lag wegen ihrer hohen Resonanzenergie von 6.385 MeV außerhalb des zugänglichen Energiebereichs der übrigen Göttinger Beschleuniger.

Die Arbeit lässt sich grob in zwei Abschnitte unterteilen. Der erste Teil besteht in der Beschreibung der wichtigsten Komponenten der Beschleunigeranlage und des Messplatzes (Kap. 2). Dort werden alle wesentlichen Parameter des Beschleunigers (Strahlerzeugung, Strahlströme, Energieeichung und -stabilisation) diskutiert. Da die Energieeichung des Beschleunigers nur mit Hilfe der resonanten Kernreaktionsanalyse durchgeführt werden kann, erfolgt die Beschreibung dieser Messmethode und des Low-Level Messplatzes ebenfalls im ersten Teil. 
Nach einer Beschreibung der übrigen verwendeten Messmethoden in Kap. 3 werden in Kap. 4 kurz einige grundlegende Mechanismen bei der Materialmodifikation mittels Laserstrahlung und Ionenimplantation beschrieben. Dies geschieht im Hinblick auf den in dieser Arbeit untersuchten Prozess des Laserhydrierens von kristallinem und Ionenstrahlamorphisiertem Silizium. Der Begriff „Laserhydrieren“ bezeichnet dabei die Laserbestrahlung von Materialien in einer Wasserstoffatmosphäre. Das Laserhydrieren stellt eine Verallgemeinerung des in der Göttinger Gruppe schon ausgiebig untersuchten „Lasernitrie-

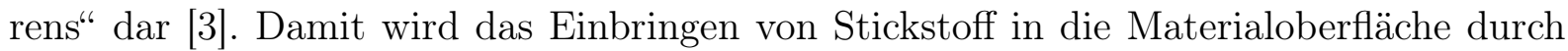
Laserbestrahlung der Probe in Stickstoffatmosphäre bezeichnet.

Während ein erhöhter Wasserstoffgehalt in Metallen meist unerwünscht ist („WasserstoffVersprödung"), ist ein erhöhter Wasserstoffgehalt in Halbleitern wegen der positiven Beeinflussung der elektronischen Eigenschaften zum Teil erwünscht.

Nachdem in Kap. 2 die Kernreaktionsanalyse lediglich als Hilfsmittel zur Kalibrierung und Optimierung des Beschleunigers verwendet wurde, schließt sich mit Kap. 6 eine Anwendung der NRA-Methode zur Messung von Wasserstofftiefenprofilen laserhydrierter Titanund Siliziumproben an.

Hierbei standen zwei Fragen im Vordergrund. Zum einen wurde untersucht, ob es durch den Prozess des Laserhydrierens überhaupt zu einem messbaren Wasserstoffeintrag in die bestrahlten Proben kommt. Und zweitens konnte die Frage beantwortet werden, ob es mittels Laserhydrieren gelingt, polykristallines Silizium mit einem Wasserstoffgehalt von mehr als 10 at\% in einem einzigen Schritt zu erzeugen. Diese Anforderungen werden an das Basismaterial zur Herstellung von TFT-Bauelementen gestellt. 


\section{Neuaufbau Beschleuniger und NRA-Messplatz}

Im Verlauf der vorliegenden Doktorarbeit wurde ein 3 MV-Pelletron 9-SDH2 der Firma NEC in der ehemaligen Zyklotron-Halle des II. Physikalischen Instituts der Universität Göttingen aufgebaut und in Betrieb genommen. Die folgenden Abschnitte beschreiben die Installation und Funktion der grundlegenden Komponenten der auf den Namen MaRPel (Materials Research Pelletron) getauften Anlage (s. Abb. 1).

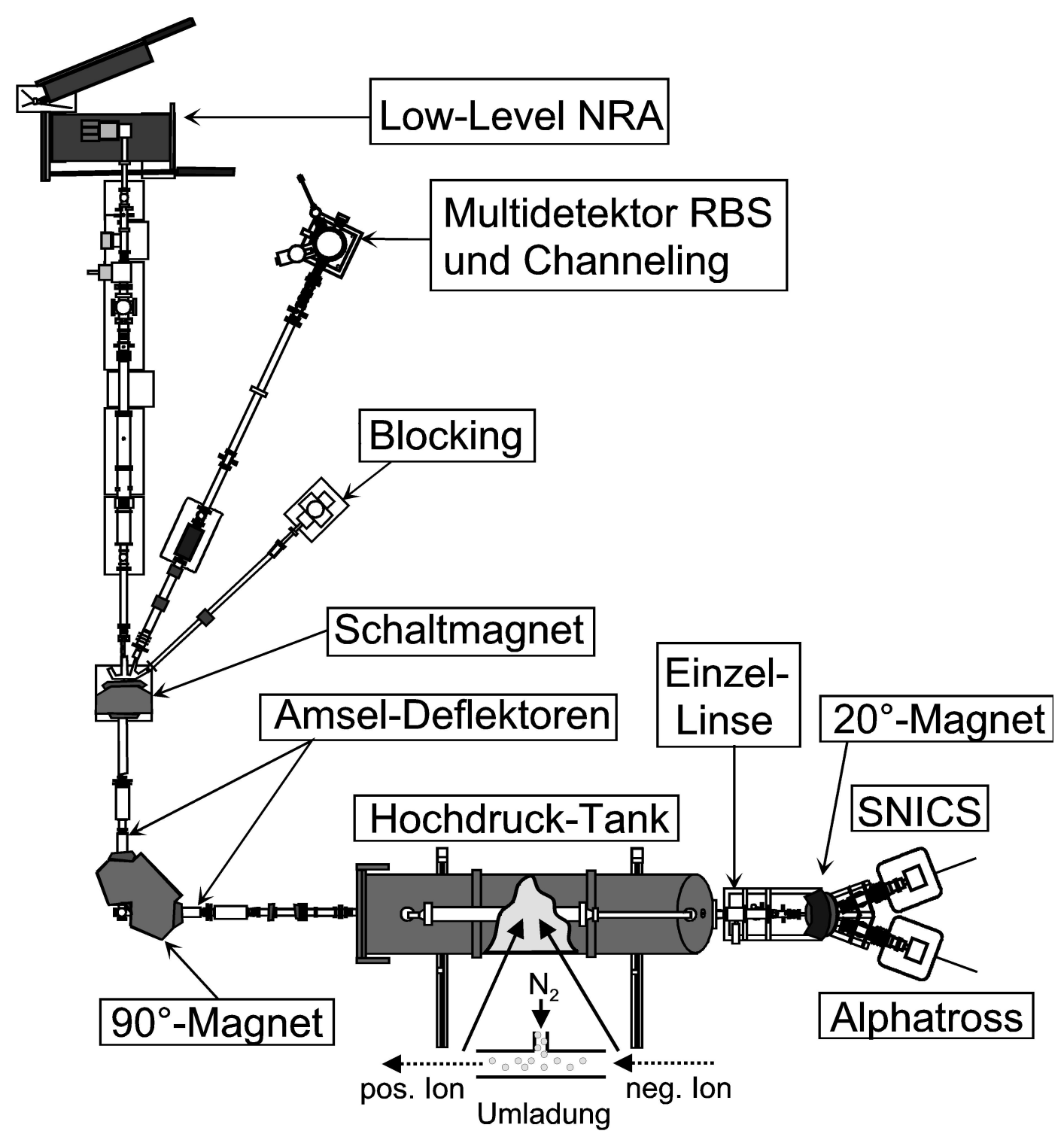

Abbildung 1: Wesentliche Komponenten des MaRPel-Beschleunigerlabors 
Zur besseren Orientierung in den folgenden Abschnitten folgt an dieser Stelle eine Beschreibung der generellen Funktionsweise des MaRPel-Beschleunigers.

In Abb. 1 sind rechts unten die beiden Ionenquellen „SNICS“ und „Alphatross" eingezeichnet. Hier werden die für den Pelletronbetrieb notwendigen negativen Ionen erzeugt und nach einer Vorbeschleunigung von bis zu $60 \mathrm{kV}$ durch den $20^{\circ}$-Quellenmagneten auf die Strahlachse des Beschleuniger-Tanks gelenkt. Nach einer ersten Fokussierung durch die Einzel-Linse am Tank-Eingang werden die negativen Ionen zum positiv geladenen Mittelteil des Hochdruck-Tanks beschleunigt.

Im Strippergas-Kanal findet die Umladung der einfach negativ geladenen Ionen durch Stöße am Strippergas $\left(\mathrm{N}_{2}\right)$ statt. Die nun bis zu dreifach positiv geladenen Ionen werden auf der zweiten Hälfte der Beschleunigerröhre erneut in Richtung des auf Erdpotenzial liegenden Tankendes beschleunigt. Aufgrund dieser zweifachen Nutzung des beschleunigenden Potenzials wird dieser Beschleunigertyp auch als „Tandem-Beschleuniger" bezeichnet. Mit dem Magnetfeld des $90^{\circ}$-Magneten wird bei bekannter Ionenmasse die Energie des Ionenstrahls eingestellt. Mit Hilfe des darauf folgenden Schaltmagneten wird das Strahlrohr ausgewählt, in das der Ionenstrahl für die Experimente gelenkt werden soll. Die daran anschließenden Strahlrohre sind jeweils mit magnetischen und elektrostatischen Fokussierelementen ausgerüstet. Am Ende des Null-Grad-Strahlrohrs befindet sich der Low-Level Messplatz zur resonanten Kernreaktionsanalyse, an dem die Experimente zur Wasserstofftiefenprofilierung der vorliegenden Arbeit durchgeführt wurden. Am Ende des $25^{\circ}$-Rohrs befindet sich der Messplatz für die Multi-Detektor-Rutherford-Rückstreu-Spektroskopie und am Ende des $45^{\circ}$-Rohrs ist die Kammer für Blocking-Experimente aufgebaut.

\subsection{Umzug und Neuinstallation}

Am 03.03.1998 unterschrieben die Universität Göttingen, vertreten durch die Kanzlerin Dr. Frost und das Max-Planck-Institut für Kernphysik Heidelberg, vertreten durch Prof. Dr. Mauersberger, die Schenkungsurkunde über einen 3 MV Pelletron-Beschleuniger, mitsamt einem Ionenstrahllabor, das bis dahin im Halbleiterlabor des MPI für Kernphysik in Heidelberg aufgebaut war (Abb. 2). Mit dem Schenkungsvertrag war die Verpflichtung verbunden, das Labor im Anschluß an den Umzug von Heidelberg nach Göttingen sobald wie möglich wieder in Betrieb zu nehmen.

Während in Göttingen durch die Demontage des Zyklotrons im Winter 1998 Platz für das neue Labor geschaffen wurde, begann in Heidelberg die Katalogisierung aller Komponenten des Beschleunigersystems (Vakuumsystem und Steuerelektronik), wie auch der gesamten Messtechnik. Innerhalb von drei Monaten (Januar-März 1999) wurde das gesamte Labor demontiert, transportfähig gemacht und mit insgesamt vier VierzigtonnenSattelschleppern nach Göttingen überführt.

Während des Abbaus in Heidelberg waren über insgesamt sechs Wochen hinweg stets

mindestens vier Mitarbeiter des II. Physikalischen Instituts damit beschäftigt, die Beschleunigeranlage zu demontieren.

Zunächst wurden alle drei Strahlrohre der Messhalle (s. Abb. 2) und die drei daran befindlichen Experimentaufbauten (Low-Level Kernreaktionsanalyse, Multidetektor-RutherfordRückstreuspektroskopie und hochauflösenden Rückstreuspektroskopie) zerlegt und sachgerecht verpackt. Insbesondere die Hochvakuum-Komponenten (z.B. Turbo- und Kryo- 


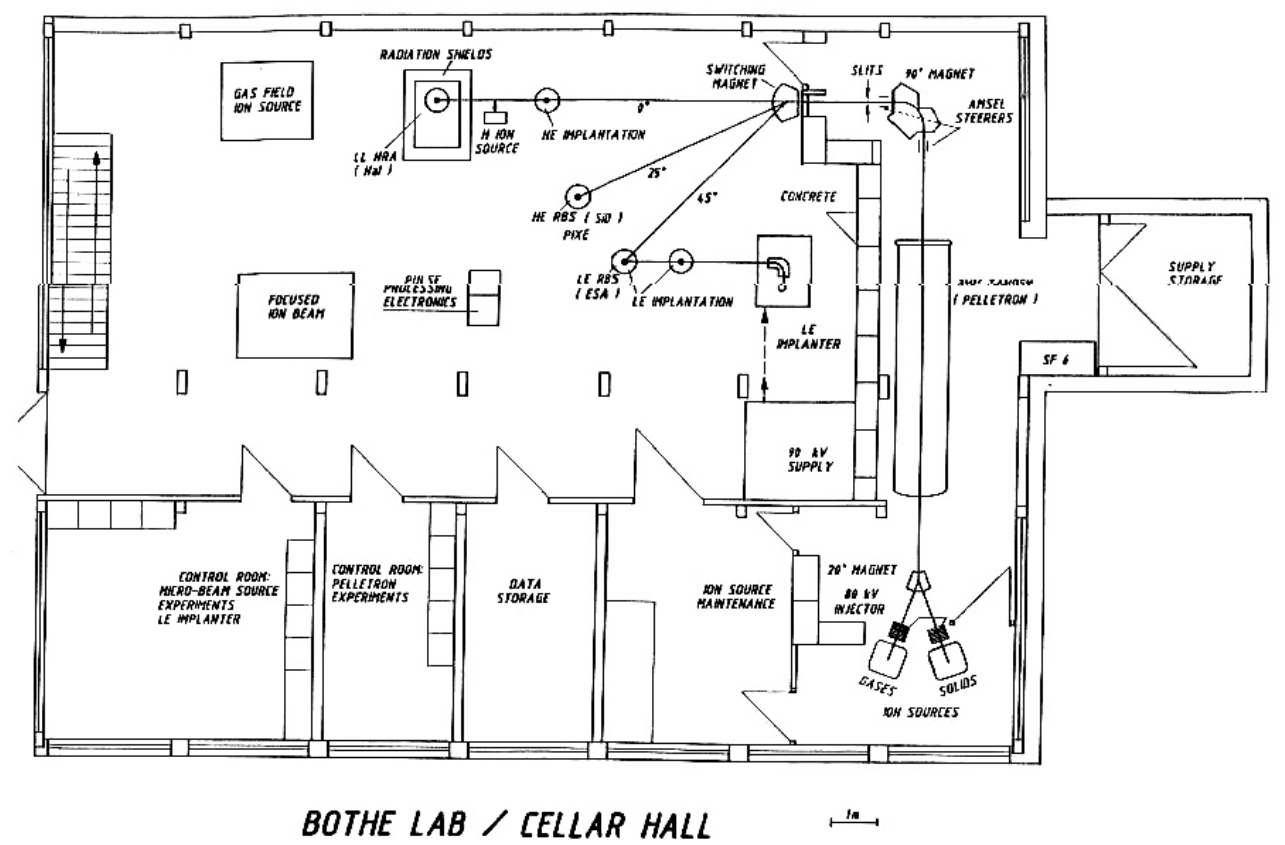

Abbildung 2: Ehemaliges Halbleiterlabor im Bothe-Labor des MPI für Kernphysik Heidelberg

pumpen) machten die Verwendung einer ausgesprochen stoßsicheren Verpackung nötig. Nach der Demontage des Vakuumsystems des Beschleunigerbereichs folgte die Deinstallation sowohl der Messelektronik, als auch der gesamten Steuereinheit des Beschleunigers. Zuletzt wurden alle diejenigen Elemente zerlegt, für die ein Schwertransport nötig war. Im einzelnen sind dies: die Eisenabschirmung des Low-Level Messplatzes, die drei großen Magnete (Quellenmagnet, Schaltmagnet und Massenseparator) und natürlich der Hochdrucktank des Beschleunigers.

Nach dem Transport durch die Fa. Hüttinger wurden die Komponenten des Vakuumsystems umgehend in der renovierten ehemaligen Zyklotronhalle zusammengefügt und justiert. Dabei wurden wiederum die schwersten Komponenten (Tank, Magnete, Eisenabschirmung) als erstes an ihren endgültigen Platz gebracht.

Besonders brisant war die Frage, ob die eigentliche Beschleunigerröhre, die aus einer freitragenden Säule aus verklebten Metall- und Keramikteilen mit einem Gesamtgewicht von zwei Tonnen besteht, den Umzug unbeschadet überstanden hatte. Für den Transport war diese Säule von einem LKW-Schlauch in der Mitte des Tanks umgeben und dadurch gestützt worden. Für den ersten Vakuumtest wurden an beiden Enden der Säule mobile Hochvakuum-Pumpstände angeflanscht und nach fünf Tagen wurde ein Vakuum besser $2 \times 10^{-2}$ Pa erreicht. Dies war der erste wichtige Teilerfolg des Umzugs.

Nach einer ersten Wartung des Tankinneren, bei der u.a. alle 600-M $\Omega$-Widerstände der Potenzialflächen geprüft und die beiden Ketten Pellet für Pellet gesäubert worden waren, mussten nun die Justierhilfen und Wandmarken installiert werden. Mit Hilfe eines Theodoliten wurden in einem ersten Schritt die Strahlachsen im Raum festgelegt und die entsprechenden Wandmarken gesetzt. Dann wurden die Wandmarken mit Halterungen für Fernrohre versehen, mit denen in der Folge die exakte Ausrichtung der Strahlrohre und Vakuumkammern vorgenommen werden konnte.

Besonders hohe Ansprüche an die Genauigkeit wurden auch bei der exakten Ausrichtung 
des Beschleuniger-Tanks gestellt. Da der Strippergas-Kanal im Inneren der Beschleunigerröhre mit einer Länge von $0.5 \mathrm{~m}$ einen Durchmesser von nur $7 \mathrm{~mm}$ aufweist, war eine millimetergenaue Positionierung des 4 t schweren Tanks unabdingbar. Auch beim Massenseparator $\left(90^{\circ}\right.$-Magnet) war eine exakte Ausrichtung wichtig, da mit seiner Position auch die Strahlachse in Richtung der Messhalle festgelegt war. Eine zusätzliche Komplikation bestand in der Tatsache, dass die Magnetkammer im Vergleich zum Heidelberger Labor um $180^{\circ}$ gedreht aufgebaut werden musste (vgl. Abb. 1 und Abb. 2). Diese Kammer ist wegen der hohen Belastung durch Strahlenschäden der defokussierten Ionenmassen mit einer Wasserkühlung versehen, die nach dem Umzug leider Lecks in den Vakuumbereich hinein aufwies. Dieser Umstand machte eine mehrfache Demontage des gesamten Massenseparators zur Reparatur des Kühlkreislaufs nötig.

Nachdem auch die Justierelemente für die Strahlrohre in der Messhalle angebracht waren, wurden die Strahlrohre im Bereich Beschleuniger-Massenseparator-Schaltmagnet vervollständigt. Danach konnte die Strahlenschutz-Mauer aufgebaut werden, die den Beschleunigerbereich von der Messhalle trennt. Da im Bereich um den Massenseparator und am Hochenergie-Ende des Tanks mit erhöhter Strahlung (Röntgenstrahlung, Neutronen) gerechnet werden muss, wurden die Türen zur Messhalle mit $8 \mathrm{~cm}$ dickem Polyethylen und Bleiplatten der Dicke $3 \mathrm{~mm}$ versehen.

Nachdem für die beiden Einschussrichtungen der Ionenquellen ebenfalls die Justierelemente im Quellenbereich montiert waren, konnte mit der Ausrichtung des Quellenmagneten auch der Strahlrohrbereich zwischen dem Quellenmagnet und dem Niederenergie-Ende des Beschleuniger-Tanks fertiggestellt werden.

Der Aufbau der beiden Ionenquellen, Alphatross und SNICS, machte die Installation einer 110 V-Spannungsversorgung notwendig. Da beide Ionenquellen im Strahlbetrieb auf einem Potenzial von bis zu 60 kV liegen, musste auch eine Plexiglas-Absperrung des Quellenbereichs neu aufgebaut werden. Bei einer letzten Wartung des Hochdrucktanks wurde das Tankinnere noch einmal komplett gesäubert und das Molekularsieb des Systems zur Entwässerung des Schutzgases gewechselt. Bevor der Hochdruck-Tank dann mit dem Schutzgas Schwefelhexafluorid befüllt werden konnte, wurde er ca. eine Woche lang bis auf einen Enddruck von $5 \times 10^{2} \mathrm{~Pa}$ evakuiert.

Den Abschluß fanden die Aufbaumaßnahmen in der Installation des Kontrollraums. Von hier aus können sämtliche Parameter des Beschleunigers, wie die Quellenparameter, die Strahlströme im gesamten Strahlverlauf, die Vakua in allen Abschnitten der Strahlführung uvm. ferngesteuert bzw. überwacht werden. Als besonders nützlich haben sich pneumatisch ins Strahlrohr einfahrbare Quarze herausgestellt, mit deren Hilfe die Strahlform optisch kontrolliert werden kann (s. Abb.3). Mit kleinen Videokameras wird die Ionoluminiszens des einfallenden Ionenstrahls aufgenommen und kann auf einem Monitor im Kontrollraum zur Strahlfokussierung beobachtet werden. Auf den Aufbau des $0^{\circ}$-Strahlrohres und der darin enthaltenen ionenoptischen Elemente wird in Abschnitt 2.2.2 genauer eingegangen.

War der Umzug glücklicherweise komplett ohne Beschädigung der zum Teil äußerst fragilen Komponenten verlaufen, so wurde beim Wiederaufbau jedoch in sehr vielen Fällen festgestellt, dass Service-Arbeiten dringend notwendig waren. So wurden beispielsweise die beiden Ionenquellen (Alphatross und SNICS) zum Teil mehrfach überarbeitet und im Falle der Alphatross sogar teilweise neu konzipiert. Dies ist in Abs. 2.1.1 beschrieben. 


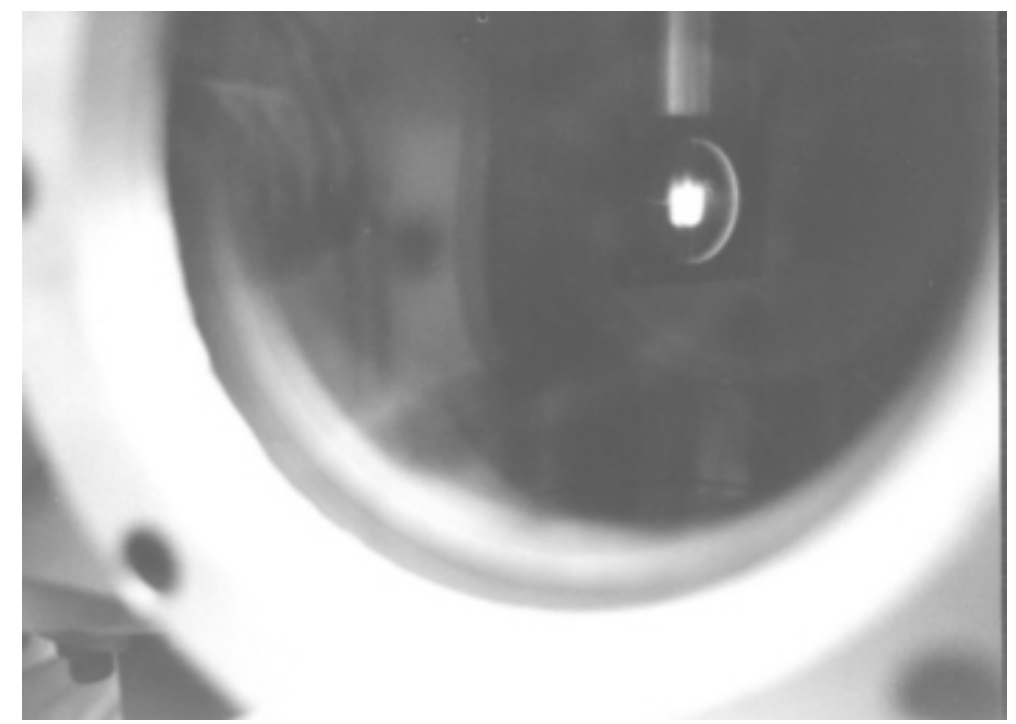

Abbildung 3: Erster Strahl am 29.09.1999.

Nach der Inbetriebnahme der Ionenquellen und des Beschleunigertanks wurde in Erfüllung des Schenkungsvertrages am 29.09.1999 der erste massenseparierte Heliumstrahl des neuen Beschleunigerlabors beobachtet (Abb. 3).

Im Strahlbetrieb konnten nun die wichtigsten Systemparameter bestimmt und optimiert werden. Alle Parameter, die Einfluss auf die Energie des Ionenstrahls haben, lassen sich mit der Methode der Kernreaktionsanalyse bestimmen, denn diese ionenstrahlanalytische Methode ist durch die dabei genutzten scharfen Kernresonanzen ein äußerst präziser Energiemaßstab [4, 5]. Diese Methode wird in Abschnitt 2.2 ausführlich beschrieben.

So machte die Energieeichung des $90^{\circ}$-Massenseparators (s. Abschnitt 2.3.2) die vorherige Inbetriebnahme des Messplatzes zur Kernreaktionsanalyse erforderlich. Diese Messapparatur, die das Hauptinstrument der vorliegenden Arbeit darstellt, war schon durch ihre 13 t schwere Untergrundabschirmung aus altem Eisen ein besonders ,schwerer" Teil des Umzugs. Da der zugehörige Messrechner und die verwendete Software veraltet waren, wurde auch die Messdatenerfassung inklusive der aktiven Untergrundunterdrückung erneuert, wie in A bschnitt 2.3.4 genauer ausgeführt wird.

Haupthindernisse beim baldigen Regelbetrieb des Beschleunigerlabors waren zum einen Komplikationen mit den beiden Ionenquellen und zum anderen die Energiestabilisierung des Ionenstrahls. Das 3 MV-Pelletron der Fa. NEC beinhaltet eine Steuerungseinheit, die sowohl hochfrequente als auch niederfrequente Schwankungen des Hochspannungspotenzials ausgleicht. Diese Regelelektronik, die zum einen an verschiedenen Stellen aktiv in die Pelletronsteuerung eingreift, zum anderen für eine effektive und korrekte Messung unbedingt erforderlich ist, war an verschiedenen Punkten defekt und darüber hinaus schlecht kalibriert. Auf die Energiestabilisierung in Verbindung mit den Amsel-Deflektoren wird aus diesem Grund in Abs. 2.1.3 sehr detailliert eingegangen. 


\subsubsection{Ionenquellen}

In diesem Abschnitt werden die beiden Ionenquellen beschrieben, mit deren Hilfe die für den Tandembeschleuniger notwendigen negativen Ionen erzeugt werden. Die negativen Ionen werden aus dem Quellenbereich durch eine Hochspannung von bis zu $60 \mathrm{KeV}$ beschleunigt, vom 20-Grad-Quellenmagneten auf die Beschleunigerachse gelenkt und schließlich vom Niederenergie-Eingang der Beschleunigerröhre aus weiter beschleunigt.

\section{Feststoff-Ionenquelle (SNICS)}

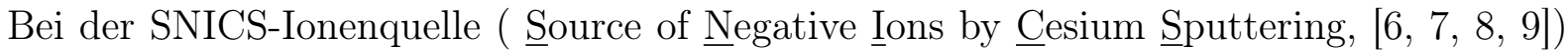
werden negative Ionen dadurch erzeugt, dass die zu beschleunigende Spezies mittels Cäsium-Ionen aus einer heißen Kathode gesputtert wird (s. Abb. 4). Cäsium wird zunächst

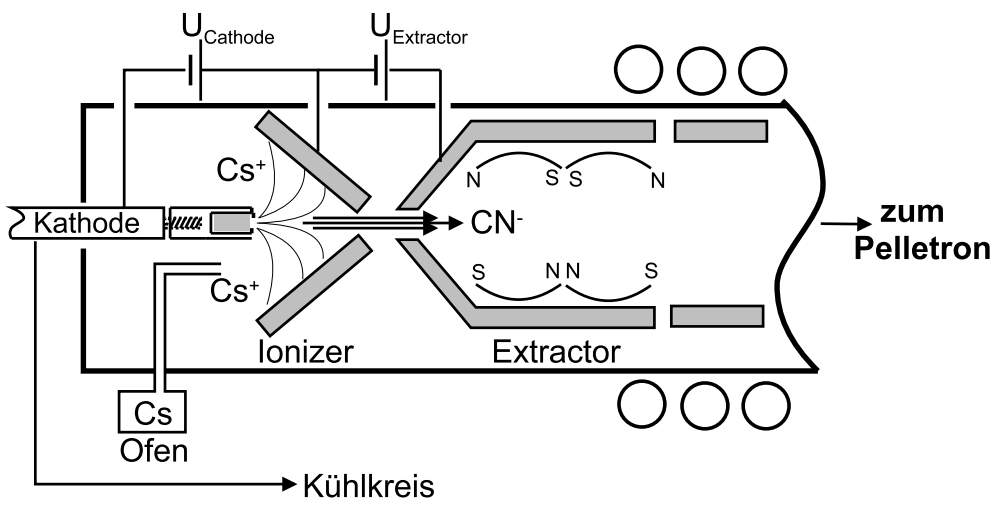

Abbildung 4: Prinzipskizze der SNICS-Ionenquelle

mit einem Ofen verdampft $\left(\mathrm{T}_{\text {dampf }}(\mathrm{Cs})=37^{\circ} \mathrm{C}\right)$ und in die Ionenquelle eingeleitet. Dort schlägt sich ein Teil des Cäsiums am Tantal-Ionizer nieder. Durch Oberflächenionisation an der heißen Ta-Oberfläche entstehen $\mathrm{Cs}^{+}$-Ionen, die durch die Kathoden-Spannung $\mathrm{U}_{\text {Cathode }}$ zur kalten Kathode hin beschleunigt werden. Auf der Kathoden-Oberfläche schlägt sich ebenfalls Cäsium nieder. Durch diese Oberflächen Cs-Schicht und den in der Kammer vorhandenen Cs-Dampf kommt es zur Bildung eines metastabilen negativ geladenen Zustands der von der Kathode abgesputterten Atome. Mittels der Extraktionsspannung $\mathrm{U}_{\text {Extractor }}$ werden die negativen Ionen von der Kathode abgezogen und in Richtung der Vorbeschleunigungsstrecke geleitet. Mit dieser Ionenquelle werden Strahlströme von $\mathrm{I}_{\text {Quelle }} \approx 30 \mu \mathrm{A}$ für Protonen und $20 \mu \mathrm{A}$ für $\mathrm{C}^{15} \mathrm{~N}^{-}$-Ionen erreicht.

Beim Betrieb der SNICS muss die Sputter-Kathode ständig gekühlt werden. Die zugehörige Wasserkühlung ist mit einem Sekundärkreis ausgestattet, der mit deionisiertem Wasser betrieben wird. Dieser Sekundärkreis führt in den Hochspannungsbereich der Quellen hinein und muss daher isolierendes Kühlmittel verwenden. Während des Probebetriebes kam es jedoch immer wieder zu Hochspannungs-Überschlägen in die Schlauchleitungen und in der Folge zu Kühlwasserlecks. Dieses Problem konnte erst durch eine neue Quellenkühlung endgültig behoben werden, die komplett innerhalb des Hochspannungsbereichs arbeitet. Auch der fehlerhafte Betrieb des Cs-Ofens führte in der Anfangsphase häufig dazu, dass die Ionenquelle über Wochen hinweg ausfiel. War durch Ausfall der Kühlung, durch ein Vakuumleck oder durch eine fehlerhafte Ofentemperatur Cs-Dampf in den Bereich der 
Isolatoren der Vorbeschleunigungsröhre gelangt, so musste jeweils das komplette Vakuumsystem der SNICS demontiert werden. Durch die Bedampfung mit Cs wurden die Keramik-Isolatoren leitend und mussten intensiv gereinigt werden. Da freies Cs an Luft explosionsartig reagiert, muss jeder Ausbau der Quelle unter Ar-Schutzgas erfolgen.

Desweiteren stellte sich heraus, dass die Zusammensetzung des Sputtermaterials der Kathoden einen sehr großen Einfluss auf die erreichbaren Strahlströme hat. So wurde beispielsweise der Strahlstrom aus einer KCN-Kathode durch die Beimischung von Ag-Pulver verdoppelt.

\section{Gas-Ionenquelle (Alphatross)}

Zur Herstellung von negativen Ionen gasförmiger Stoffe (speziell He) steht die AlphatrossIonenquelle zur Verfügung [10, 11, 12]. In einer Quarzröhre wird zunächst mittels eines Hochfrequenz-Feldes ein Plasma der zu beschleunigenden Gasionen gezündet. In einem ersten Schritt werden die positiven Ionen durch die Probe-Spannung U $\mathrm{U}_{\text {Probe }}$ aus der Quarzröhre in die daran anschließende Umladungskammer beschleunigt (s. Abb. 5). In dieser Kammer, die mit Rb-Dampf gefüllt ist, findet die Umladung der Gasionen in den negativen Ladungszustand statt. Die Extraktionsspannung $\mathrm{U}_{\text {Extr }}$ beschleunigt die (negativ geladenen) Gasionen aus der Umladungskammer in Richtung der Vorbeschleunigungsstrecke. Beim Betrieb der Alphatross hat sich herausgestellt, dass die Temperatur der gekühlten Flansche an der Eintritts- und Austrittsseite dieser Kammer einen großen Einfluss auf die erreichbaren Strahlströme hat. Außerdem zeigte sich bei Versuchen mit Helium-Ionen, dass für die Umladung im Gasplasma ein sehr gutes Vakuum in der Quarzröhre nötig ist. Schon bei Restgas-Drucken im Bereich von $10^{-7}$ Pa war eine deutliche Verfärbung des Plasmas zu erkennen. Solange der Restgasdruck (vor allem Wasserdampf) zu hoch und die Plasmafarbe nicht „ozeangrün“ (NEC) war, konnte kein $\mathrm{He}^{-}$-Strahlstrom gemessen werden. Um den Restgasdruck immer weiter herabzusenken und vorhandene Vakuumlecks

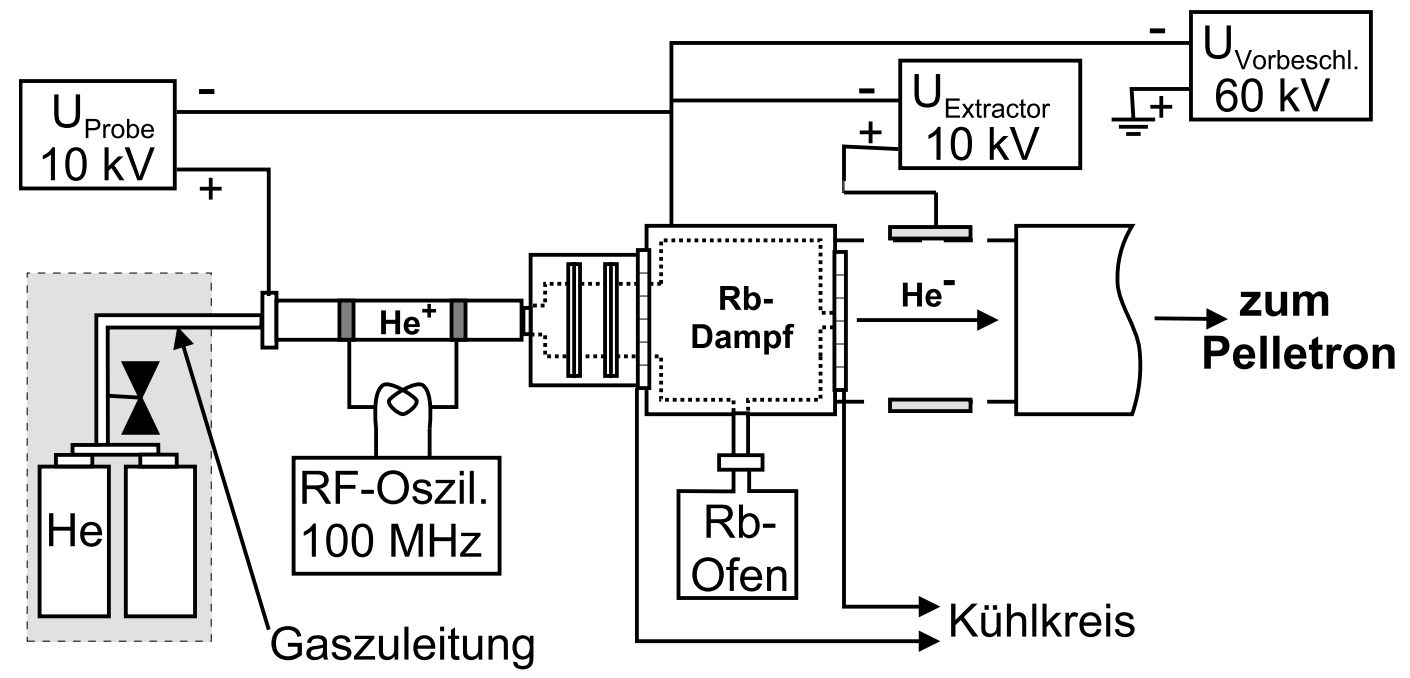

Abbildung 5: Skizze der Alphatross-Ionenquelle

zu schließen, wurde das Gaszuleitungssystem mehrmals umgearbeitet und vereinfacht. Beim ursprünglichen Aufbau der Alphatross bestand die Gaszuleitung für das Plasma (s. Abb.5) aus einem Teflonschlauch. Die Gasleitung mußte elektrisch isolierend sein, da 
die Eintrittsöffnung der Quarzröhre gegenüber der restlichen Quelle auf einem erhöhten elektrischen Potenzial liegt $\left(\mathrm{U}_{\text {Probe }}\right)$. Es zeigte sich, daß dieser Teflonschlauch und das daran anschließende Regelventil Vakuumlecks im Bereich von $10^{-6} \mathrm{~Pa}$ enthielten. Zusätzlich wurden Spannungsüberschläge innerhalb des Teflonschlauchs beobachtet, die keine stabile Extraktion der Ionen aus dem Gasplasma erlaubten. Erst durch den Austausch des Regelventils und den Einsatz von Edelstahl-Gasleitungen konnte der Restgas-Druck deutlich verbessert werden. Dadurch wurde ein Umbau der Halterung der Gasregelung und der Gasflaschen nötig, denn diese mußten nun gegenüber der restlichen Quelle elektrisch isoliert werden. Die neue Halterung wurde aus Plexiglas gefertigt und löste die genannten Probleme.

Zusätzlich stellte sich heraus, dass sich auch im Regelbetrieb Rb-Dampf an der Innenseite der Quarzröhre absetzt und mit fortschreitender Betriebsdauer den Durchgang zur Umladekammer verstopft. Dadurch sind nach ca. einer Woche Strahlbetrieb Servicearbeiten un-

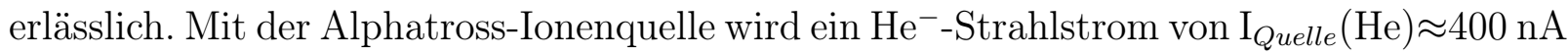
erreicht.

\subsubsection{Beschleuniger-Tank}

Im Beschleuniger-Tank, der mit Schutzgas $\left(\mathrm{SF}_{6}\right)$ unter hohem Druck (bis 6 bar) gefüllt ist, erhalten die vorbeschleunigten Ionen ihre Endenergie von bis zu $3 \mathrm{MeV}$ pro Ladungszustand. Der Hochdrucktank enthält eine frei tragende Säule, die aus Metall-Potenzialflächen

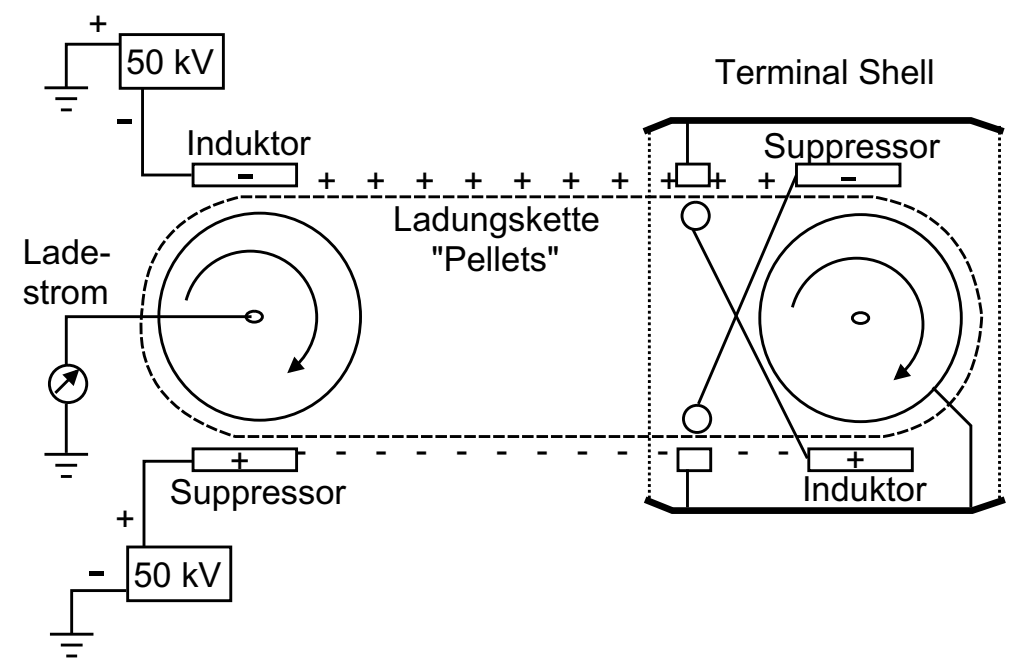

Abbildung 6: Prinzipskizze des Ladungssystems. Das Feld der negativ geladenen InduktorElektrode bewegt die Elektronen der Pellets auf das geerdete Antriebsrad, so dass eine positive Ladung der Pellets resultiert. Die Kette transportiert diese positive Ladung zum Hochspannungsterminal, wo der negativ geladene Suppressor Spannungsüberschläge verhindert. Erst nach dem Verlassen des Feldbereichs wird die positive Ladung auf das Terminal-Laufrad aufgebracht. Auf dem Rückweg wird umgekehrt negative Ladung von der Terminal-Shell forttransportiert (nach [13]).

besteht die im Wechsel mit Keramikzylindern elektrisch isolierend miteinander verklebt sind. In der Mitte dieser Säule befindet sich die sogenannte Terminal-Shell, die im Betrieb 
auf ein Potenzial von bis zu $3 M V$ gelegt werden kann. Die Aufladung der Terminal-Shell gelingt über zwei Metallketten, deren einzelne Glieder gegeneinander isoliert sind (sog. Pellets). Die Ketten laufen mit einer Geschwindigkeit von $12 \mathrm{~m} / \mathrm{s}$ und tragen die positive Ladung, die am Hochenergieende der Säule von den Induktoren berührungsfrei auf die Pellets influenziert wird, auf die Terminal-Shell (s. Abb. 6).

Nachdem die vorbeschleunigten negativen Ionen den Quellenbereich verlassen haben, werden sie durch eine Einzellinse am Niederenergie-Ende des Beschleuniger-Tanks fokussiert und in die Beschleuniger-Röhre eingeschossen. In einem ersten Schritt werden die negativen Ionen innerhalb der Säule zur positiv geladenen Terminal-Shell beschleunigt. Im Bereich der Terminal-Shell befindet sich ein Strippergaskanal, in den Stickstoff unter einem Druck von $6 \times 10^{-6} \mathrm{hPa}$ eingeleitet wird. Durch Stöße am Strippergas findet der Umladungsprozess der negativen Ionen über den neutralen Zustand bis hin zu dem dreifach positiven Ladungszustand statt (im Falle von Ionen mit $z \geq 3$ ). Im zweiten Schritt wird die Potenzialdifferenz zum Hochenergieende des Beschleunigers durchlaufen, wodurch die Ionen noch einmal bis zu $3 \mathrm{MeV}$ pro Ladungszustand erhalten. Aus dieser zweistufigen Beschleunigung der Ionen rührt der Name „Tandembeschleuniger" her.

Die Erzeugung eines ${ }^{15} \mathrm{~N}$-Strahls stellt dabei einen Spezialfall dar. Da es aufgrund der Elektronegativität des Stickstoffs nicht möglich ist, negative Stickstoff-Ionen zu erzeugen, werden in der SNICS-Ionenquelle negativ geladene $\mathrm{C}^{15} \mathrm{~N}^{-}$-Moleküle erzeugt und in den Beschleunigertank eingeschossen.

\subsubsection{Energiestabilisierung}

Wie jeder Tandembeschleuniger ist auch das 3 MV NEC Pelletron mit einem sogenannten Terminal Potential Stabilizer (TPS) ausgerüstet [14, 15, 16. Dieses System zur Stabilisierung der beschleunigenden Hochspannung und damit der Strahlenergie arbeitet folgendermaßen:

Zur Regelung des beschleunigenden Potenzials lässt sich in der Mitte des Tanks die so genannte Corona einfahren. Dabei handelt es sich um einen Aufbau, bestehend aus drei Edelstahl-Nadeln, über welche eine stehende Entladung von der Terminal-Shell hin zum Erdpotenzial gezündet wird. Der über die Corona abfließende Strom lässt sich über die Gitterspannung der nachgeschalteten Leistungsdynode regeln. Für die Regelung dieser Dynode bietet das TPS zwei Modi: GVM- und Slit-Mode. Die Elemente des TPS-Regelkreises sind in Abb. 7] schematisch dargestellt. Im „GVM-Mode“ übernimmt ein RotationsVoltmeter (Generating Voltmeter, GVM) die Grundeinstellung der Hochspannung. Zum Ausgleich höherfrequenter Schwankungen des Beschleunigungspotenzials im GVM-Mode wird dem Fehlersignal des GVM das Signal zweier Kondensatorplatten (Capacitive Pickoffs, CPO), die sich an der äußeren Tankwand auf Höhe der Shell befinden, überlagert. Damit werden die Hochspannungsschwankungen auf ca. $\Delta U \leq 500 \mathrm{~V}$ olt begrenzt. Ist eine erste Stabilisierung im GVM-Mode erreicht, so wird im „Slit-Mode“ die Hochspannung durch ein Schlitzpaar am Ausgang des $90^{\circ}$-Magneten stabilisiert. Hier wird ständig der Strom der Ionen gemessen, welche auf den in Strahlrichtung gesehen linken und rechten Schlitz treffen. Im Idealfall einer stabilen Hochspannung und damit einer konstanten Strahlenergie passiert der Ionenstrahl bei festem Magnetfeld des $90^{\circ}$-Magneten genau das Zentrum des Schlitzpaars und der Strahlstrom auf den beiden Schlitzen ist gleich groß. Ist 


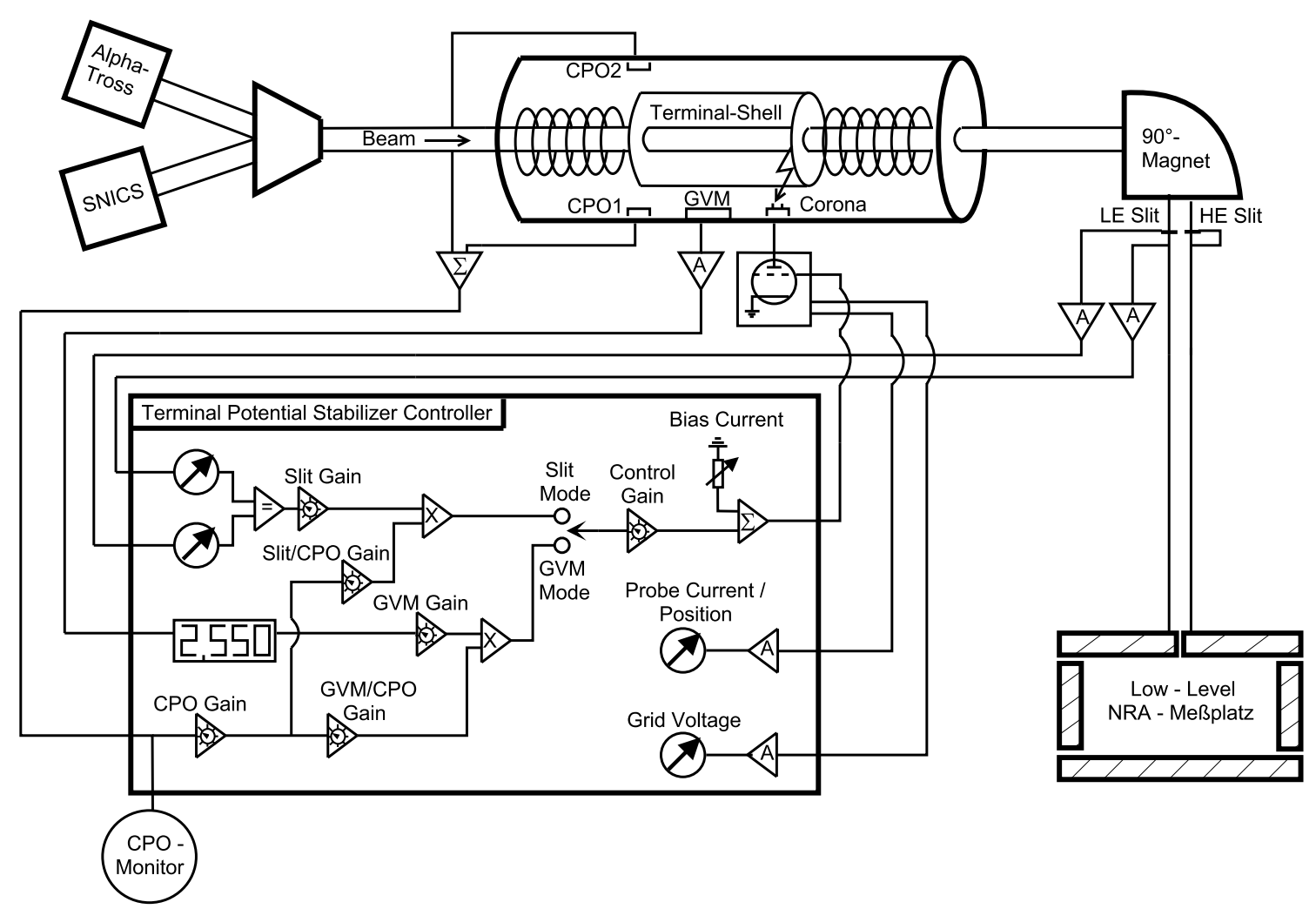

Abbildung 7: Terminal Potential Stabilizing System (nach [17])

der Strahlstrom am Hochenergie-Schlitz (links) größer, so regelt das TPS das beschleunigende Potenzial herab, d.h. die Leistungsdynode wird so eingestellt, dass mehr Strom über die Corona abfließen kann. Ist der Strom am Niederenergie-Schlitz (rechts) größer, so wird der Widerstand der Leistungsdynode durch eine höhere Gitterspannung erhöht, und somit wird das Potenzial der Terminal-Shell vergrößert. Da die Ansprechzeiten dieses Regelkreises sehr kurz sind, lassen sich damit hochfrequente Schwankungen ausgleichen, die etwa durch die Ladungsschübe der einzelnen Kettenglieder entstehen.

Es stellte sich bei den ersten Strahlzeiten von MaRPel heraus, dass dieser Regelkreis nicht richtig funktionierte und auf Grund von fehlerhaften Steuersignalen keinen stabilen Ionenstrahl zuließ, wie er insbesondere für NRA-Messungen benötigt wird. Da zunächst nicht klar war, welche Komponente dieses rückgekoppelten Regelkreises defekt war, mußten in zahlreichen Testläufen alle Bestandteile einzeln getestet werden. So wurde die Corona samt Corona-Steuerung und Leistungsdynode mehrfach auf Funktion und Hochspannungsfestigkeit geprüft. Hier musste sichergestellt werden, dass während des Betriebes eine Hochspannungsentladung zu den Corona-Nadeln konstant bestehen bleibt und keine weiteren Entladungen zur Tankhülle stattfinden. Auch das Ladungssystem aus Induktoren, Ketten und Terminal-Shell wurde mechanisch und elektrisch geprüft, um auch hier fehlerhafte Spannungsspitzen ausschließen zu können. Um unerwünschte HochspannungsDurchbrüche des Schutzgases („Poltergeist“) als Fehlerquelle auszuschließen, wurde das Molekularsieb des $\mathrm{SF}_{6}$-Recirculating-System gänzlich ausgetauscht. Daran schloß sich eine Überprüfung aller elektronischen Komponenten ausserhalb des TPS-Controlers an. Schließlich ging aus einem Vergleich der wichtigsten Steuersignale mit denen des baugleichen Pelletrons im VERA-Labor (Wien) hervor, dass nur noch der TPS-Controller 
selbst als Fehlerquelle übrigblieb. Erst eine Reparatur dieses Controllers und die Installation eines neu gebauten Einschubs zur Anzeige und Feineinstellung aller Regelparameter ermöglichte dann einen energiestabilisierten Ionenstrahl.

\subsection{Resonante Kernreaktionsanalyse (NRA)}

Nach der Inbetriebnahme der Ionenquellen und des Beschleuniger-Tanks wurde die Energieeichung des Massenseparators durchgeführt. Dabei wurden schmale Kernresonanzen als Energie-Maßstab benutzt, die auch die Grundlage der resonanten Kernreaktionsanalyse sind. Daher sollen im Folgenden die wesentlichen Elemente der resonanten Kernreaktionsanalyse besprochen werden. Diese ionenstrahlanalytische Methode bietet den Vorteil, dass mit ihrer Hilfe geringe Konzentrationen leichter Elemente (Wasserstoff, Stickstoff) isotopenspezifisch und zerstörungsfrei nachgewiesen werden können [18, 19, 20, 21].

\subsubsection{Beschreibung der Methode}

Die Grundlage der resonanten Kernreaktionsanalyse („Nuclear Reaction Analysis“, NRA) bilden schmale Resonanzen ( $1 \mathrm{eV} \leq \Gamma \leq 10 \mathrm{keV})$ im Wirkungsquerschnitt von Kernreaktionen zwischen den nachzuweisenden leichten Isotopen und einem eintreffenden Ionenstrahl. Die bei der Kernreaktion entstehenden charakteristischen Gammaquanten werden mit einem Detektor nachgewiesen. Aus der Intensität der Strahlung kann nach der Normierung auf die eingeschossene Ladung die Konzentration des jeweiligen Isotops bestimmt werden. Wird die Energie der einfallenden Ionen über die Resonanzenergie hinaus erhöht, so findet die Kernreaktion erst in einer Tiefe z statt (vgl. Abb.8), die durch die Differenz $\left(E-E_{R}\right)$ von Strahlenergie $E$ und Resonanzenergie $E_{R}$ und durch das spezifische Bremsvermögen $\frac{d E}{d x}$ im Festkörper gegeben ist:

$$
z=\frac{E-E_{R}}{\frac{d E}{d x}}
$$

Prinzipiell ist das Bremsvermögen eine Funktion der Tiefe z (und damit auch der Energie des Ionenstrahls E) sowie eine Funktion der lokalen Konzentration der nachzuweisenden Spezies. Zur Berechnung des resultierenden Bremsvermögens, das sich für eine Kombination der im Targetmaterial vorhandenen Atomsorten ergibt, muss im allgemeinen die Bragg-Regel [22] angewendet werden:

$$
S_{n_{1} \ldots n_{k}}(E)=n_{1} S_{1}(E)+n_{2} S_{2}(E)+\ldots+n_{k} S_{k}(E)
$$

Dabei bezeichnet $n_{i}$ die Konzentration des i-ten Elements und $S_{i}$ dessen Bremsvermögen. Die Werte für das Bremsvermögen können aus Tabellenwerken von Ziegler et. al [23] entnommen werden. Speziell beim Wasserstoff-Nachweis sind die Veränderungen des Bremsvermögens durch den Wasserstoff jedoch zu vernachlässigen, da der Beitrag des Wasserstoffs zum gesamten Bremsvermögen sehr klein ist. Zusätzlich wird die Wirkung durch die gleichzeitige Gitteraufweitung teilweise kompensiert [24]. 

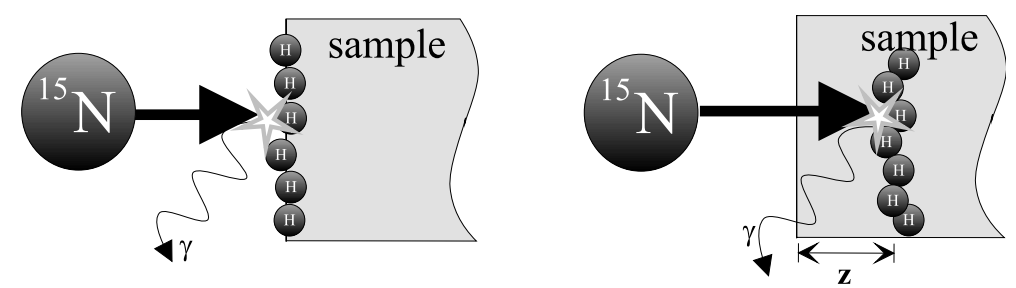

Abbildung 8: Prinzip der resonanten Kernreaktionsanalyse

Zwischen der Konzentration $c(a)$ des nachzuweisenden Isotops a und der gemessenen Zahl der Gamma-Quanten $Y_{a}$ herrscht folgende Relation:

$$
\frac{Y_{a}}{Q} \propto \frac{c(a)}{\frac{d E}{d x}}
$$

Dabei bezeichnet Q die durch den Ionenstrahl deponierte Ladung, die ein Maß für die Zahl der eingestrahlten Ionen ist. Die Proportionalitätskonstante kann durch Vergleich mit einem Standard bekannter Konzentration ermittelt werden. In der vorliegenden Arbeit wurde die Kernreaktion ${ }^{15} N(p, \alpha \gamma){ }^{12} C$ bei der Resonanzenergie $E_{R}=898 \mathrm{keV}$ mit der Breite $\Gamma=2.2 \mathrm{keV}$ [25] zur Stickstoffprofilierung sowie die Umkehrreaktion ${ }^{1} H\left({ }^{15} \mathrm{~N}, \alpha \gamma\right){ }^{12} \mathrm{C}$ bei $E_{R}=6385 \mathrm{keV}$ mit der Breite $\Gamma=1.868 \mathrm{keV}$ [26] zur Wasserstoffprofilierung benutzt. Durch die hohen Wirkungsquerschnitte von $\sigma_{898}=800 \mathrm{mb}$ und $\sigma_{6385}=1650 \mathrm{mb}$ erreicht man mit den beiden genannten Kernreaktionen eine hohe Sensitivität.

Prinzipiell müssen die einfallenden Ionen ausreichend Energie besitzen, um den CoulombWall des Targetkerns zu überwinden, damit überhaupt eine Kernreaktion ausgelöst werden kann. Die Höhe des Coulomb-Walls ist gegeben durch:

$$
V_{\text {Coul }}=\frac{1}{4 \pi \epsilon_{0}} \cdot \frac{Z_{I} Z_{T} e^{2}}{R_{I}+R_{T}} \approx \frac{1}{4 \pi \epsilon_{0}} \cdot \frac{Z_{I} Z_{T} e^{2}}{r_{0}\left(A_{I}^{1 / 3}+A_{T}^{1 / 3}\right)}
$$

Dabei bezeichnen $Z_{I}$ und $Z_{T}$ die Kernladungszahlen des Ions und des Targetkerns, $R_{I}+R_{T}$ den Wechselwirkungsradius, $A_{I}$ und $A_{T}$ deren Atommassen und $r_{0} \approx 1.3 \mathrm{fm}$.

Für die beiden oben genannten Kernreaktionen beträgt die Höhe des Coulomb-Walls $\mathrm{E}=2.24 \mathrm{MeV}$ für den Protonenstrahl bzw. $\mathrm{E}=4.79 \mathrm{MeV}$ für den ${ }^{15} N$-Strahl. Die Energien der Projektilionen liegen im Schwerpunktsystem deutlich unter diesem Wert. Der Tunneleffekt ermöglicht jedoch eine Resonanzreaktion schon bei geringerer Energie, sofern die Summe aus dem Q-Wert der Kernreaktion und der Projektilenergie im Schwerpunktsystem einem angeregten Zustand des Compoundkerns entspricht. Beide Kernreaktionen laufen nach dem Schema

$$
\begin{aligned}
{ }^{15} N+{ }^{1} H \rightarrow{ }^{16} \mathrm{O} & \rightarrow{ }^{12} \mathrm{C}^{*}+\alpha \\
{ }^{12} \mathrm{C}^{*} & \rightarrow{ }^{12} \mathrm{C}+\gamma
\end{aligned}
$$

über ${ }^{16} \mathrm{O}$ als Compoundkern ab (s.Abb. 9). Für die spätere Entfaltung des NRA-Tiefenprofils und die Bestimmung der Tiefenauflösung der Methode ist eine genaue Kenntnis der energieverbreiternden Faktoren nötig. Der natürlichen Linienbreite $\Gamma$ sind zusätzliche Beiträge aus der Energieunschärfe $\Delta E_{\text {ion }}$ des Ionenstrahls, der statistischen Energieverluststreuung (Straggling) $\sigma$, die bei der Abbremsung der Ionen im Festkörper auftritt, und aus dem Dopplereffekt $D$ der Nullpunktsschwingungen der Probenatome überlagert. Diese Beiträge sollen im folgenden quantitativ bestimmt werden. 


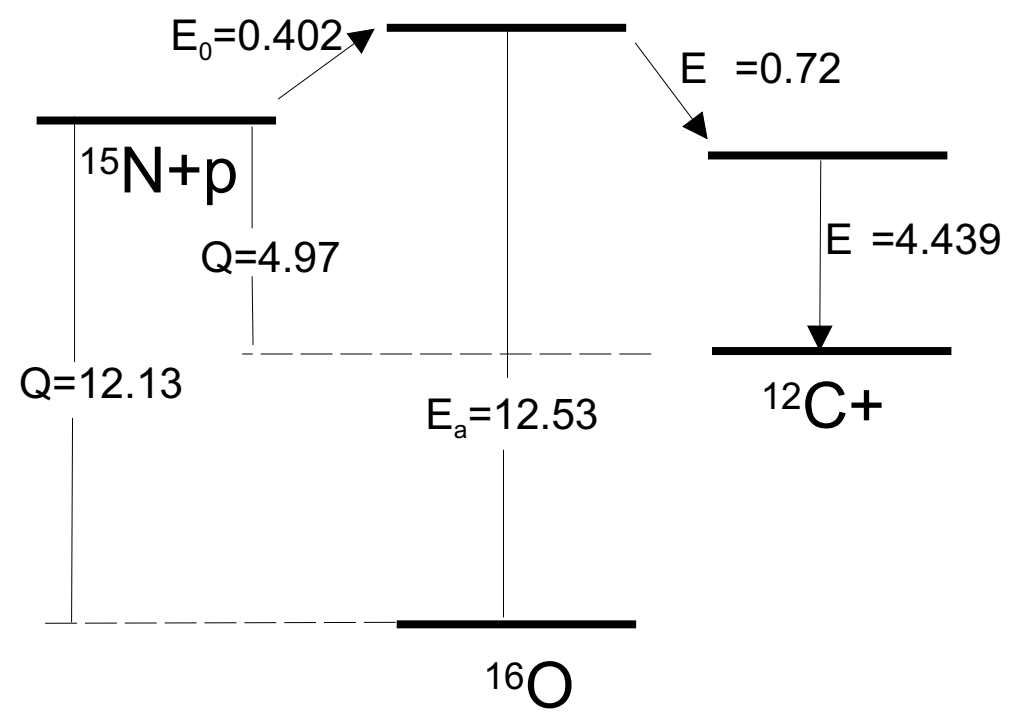

Abbildung 9: Reaktionsschema, Energien in $\mathrm{MeV}$

\section{Natürliche Linienbreite}

Die natürliche Linienbreite $\Gamma$ wird bestimmt durch die Lebensdauer des angeregten Kerns im Zwischenzustand der Reaktion. Der Resonanzquerschnitt besitzt die Breit-Wigner Form:

$$
\sigma_{\text {res }}(E)=C \cdot \frac{\Gamma_{a} \Gamma_{b}}{\left(E-E_{R}\right)^{2}+\Gamma_{r e s}^{2} / 4}
$$

wobei $E_{R}$ die Resonanzenergie, $\Gamma_{\text {res }}$ die totale Resonanzbreite und $\Gamma_{a}$ und $\Gamma_{b}$ die Partialbreiten für die Bildung und den Zerfall der Resonanz bezeichnen. Die Konstante $C$ enthält Spinquantenzahlen und die de-Broglie-Wellenlänge des Projektils.

\section{Energieunschärfe des Ionenstrahls}

Wie in Kap. 2.3.1 dargestellt, besitzt der Ionenstrahl selbst eine Energieverteilung $\Delta \mathrm{E}_{\text {ion }}$, die ebenfalls berücksichtigt werden muss. Die Werte für die sog. Maschinenbreite sind in Tab. 1 auf Seite 25 angegeben.

\section{Statistische Energieverluststreuung (Straggling) im Targetmaterial}

Für die Verbreiterung der Energieverteilung durch die statistische Natur des Energieverlustprozesses beim Durchgang der Projektilionen durch Materie existiert in der Literatur eine große Zahl unterschiedlicher Theorien [27, 28, 29, 30. Der erste theoretische Ansatz zur Berechnung der Tiefenabhängigkeit der Energieverluststreuung geht auf Bohr zurück [30. Im Grenzwert hoher Ionengeschwindigkeiten dominiert das elektronische Abbremsvermögen und das Straggling ist fast unabhängig von der Ionengeschwindigkeit. In diesem Grenzfall gilt:

$$
\sigma_{\text {bohr }}^{2}\left[\mathrm{keV}^{2}\right]=0.26 \cdot Z_{1}^{2} Z_{2} N z\left[10^{18} \mathrm{at} / \mathrm{cm}^{2}\right]
$$

Dabei bezeichnet $Z_{1}$ die Ordnungszahl des Projektilions, $Z_{2}$ die Ordnungszahl des Targetmaterials und $N z$ gibt die Tiefe im Target in der Einheit $\left[10^{18} \mathrm{at} / \mathrm{cm}^{2}\right]$ an. Eine Erweiterung dieses Ansatzes für niedrigere Ionengeschwindigkeiten und leichtere Ionen $\left(Z_{1}<Z_{2}\right)$ 


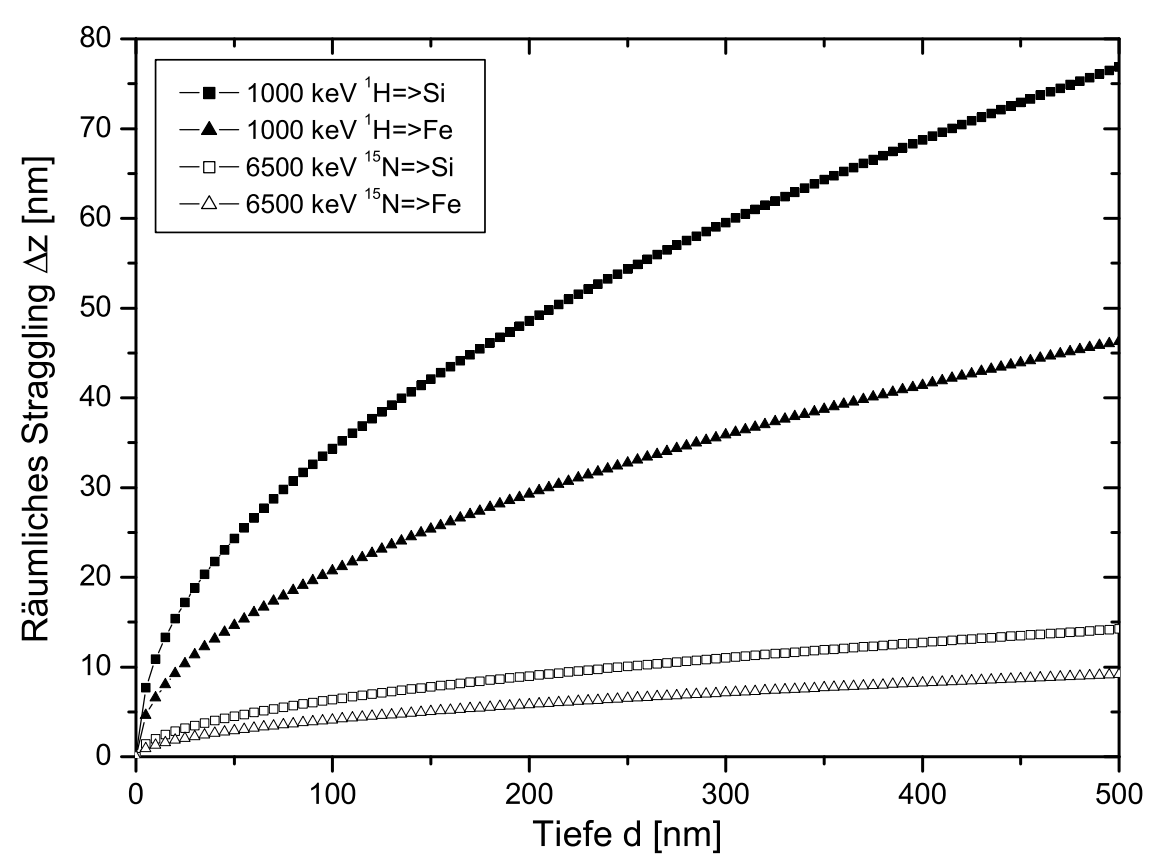

Abbildung 10: Vergleich der Ortsauflösung bei verschiedenen Analysestrahlen. Dabei wurde ein konstantes Abbremsvermögen zur Umrechnung der Energieverluststreuung in die Tiefenskala vorausgesetzt.

wurde von Lindhard und Scharff gegeben [31]:

$$
\begin{aligned}
& \frac{\sigma_{l s}^{2}}{\sigma_{\text {bohr }}^{2}}=\left\{\begin{array}{ccc}
0.5 L(x), & \text { für } & E<75 Z_{2}[\mathrm{kev} / \mathrm{amu}] \\
1, & \text { für } E \geq 75 Z_{2}[\mathrm{kev} / \mathrm{amu}]
\end{array}\right. \\
& \text { mit } L(x)=1.36 x^{1 / 2}-0.016 x^{3 / 2}, \\
& \text { und } x=\frac{E[\mathrm{keV} / \mathrm{amu}]}{25 Z_{2}}
\end{aligned}
$$

In Abb. 10 ist die Ortsaufösung in Eisen und Silizium für Ionenenergien im Bereich der verwendeten Kernreaktionen in Abhängigkeit von der Tiefe gezeigt.

\section{Bindungs-Dopplereffekt}

Durch den Dopplereffekt, der aus der stets vorhandenen thermischen Bewegung der Targetatome resultiert, wird der lorentzförmigen Energieabhängigkeit des Wirkungsquerschnitts (Gl. 5) noch ein gaußförmiger Anteil überlagert. Eine strahlparallele oder -antiparallele Bewegung der Probenatome im Potenzial der bindenden Gitteratome verändert die Geschwindigkeit der Projektilionen im Ruhesystem des jeweiligen Targetatoms. Dadurch kommt es zu einer Verbreiterung der Energieabhängigkeit des Wirkungsquerschnitts, die Dopplerverbreiterung genannt wird. Die vom Dopplereffekt herrührende Linienverbreiterung wurde von Zinke-Allmang sehr genau vermessen und quantitativ analysiert [32, 33, 34, 35. Dieser Effekt tritt jedoch besonders stark nur nahe der Probenoberfläche auf und wird mit zunehmender Tiefe vom Straggling-Effekt überdeckt. 


\subsection{2 $\quad 0^{\circ}$-Strahlrohr und Low-Level Messaufbau}

In Abbildung 11 ist der Teil des 0-Strahlrohrs des MaRPel-Labors nach dem Schaltmagneten (vgl. auch Abb.1) im Bereich der Messhalle dargestellt. Neben dem elektrostatischen

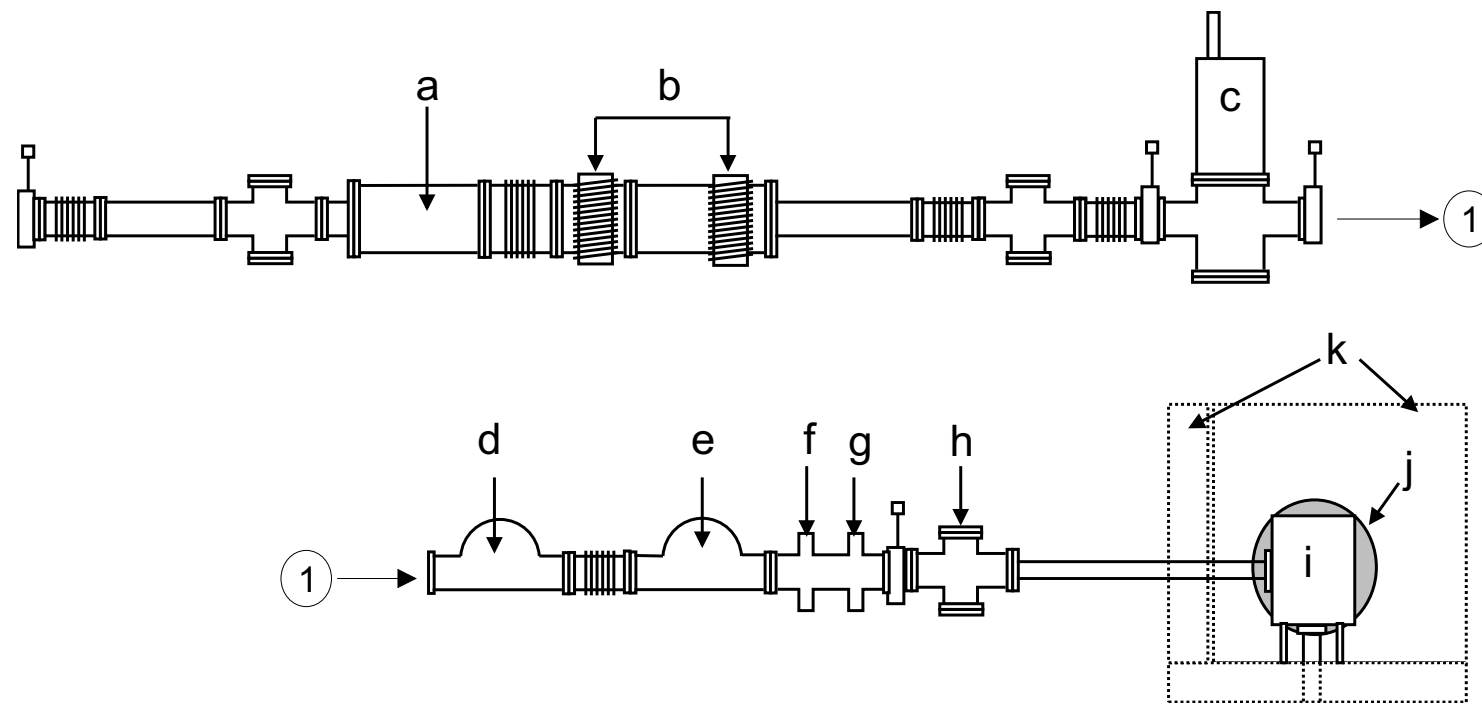

Abbildung 11: Aufbau des $0^{\circ}$-Strahlrohrs mit Low-Level Messplatz. Eingezeichnet sind ausserdem: a) Elektrostatischer Quadrupol, b) Magnetische Boxsteerer zur achsenparallelen Auslenkung des Ionenstrahls, c) Implantationsplatz, d) Ionenquelle für Niederenergie-Ionenimplantation, e) Wienfilter, f) verstellbare Schlitze, g) zweifach Viersektoren-Blende zur Kontrolle der Strahlposition während NRA-Messungen, h) Chopper zur Strahlstrommessung, i) Low-Level Messkammer, j)NaI-Detektor und k) Eisenabschirmung

Quadrupol, der zur Fokussierung des Ionenstrahls dient, enthält das $0^{\circ}$-Strahlrohr noch zwei sog. magnetische Boxsteerer, mit deren Hilfe der Ionenstrahl achsenparallel im Strahlrohr ausgelenkt werden kann. Zusätzlich ist ein Strahlverwedelungssystem eingebaut, mit dem der Ionenstrahl während der Implantation an der Hochenergie-Implantationskammer gleichmäßig über eine Fläche von $10 \times 10 \mathrm{~mm}^{2}$ verteilt werden kann. Innerhalb der Implantationskammer befindet sich eine Viersektorenblende, die ein wichtiges Hilfsmittel zur Strahlfokussierung darstellt. Daran anschließend befindet sich eine Gas-Ionenquelle im Strahlrohr, die mittels eines Stellrades seitlich in den Strahlengang eingebracht werden kann. Zusammen mit dem ebenfalls seitlich einschwenkbaren Wien-Filter wird somit zusätzlich die Möglichkeit für eine Niederenergie-Implantation direkt in die NRA-Kammer am Ende des Strahlrohrs geschaffen.

Durch eine neue Zweifach-Viersektorenblende (s. Abb. 12 mit zwei quadratischen Öffnungen von $0.25 \mathrm{~cm}^{2}$ bzw. $1 \mathrm{~cm}^{2}$ ist es nun möglich, den Strahlverlauf auch während der laufenden NRA-Messung zu kontrollieren. Kurz vor dem Eingang in die NRA-Messkammer (ca. $0.8 \mathrm{~m}$ ) befindet sich ein sogenannter „Chopper“, mit dessen Hilfe der Strahlstrom bei NRA-Experimenten gemessen wird. Es handelt sich dabei um drei isolierte Nadeln, die ständig durch den Strahl rotieren. Das Verhältnis von Eintritts- zu Nadelfläche ist so gewählt, dass der an den Nadeln gemessene Strom ein Zehntel des tatsächlichen Stroms ausmacht. 


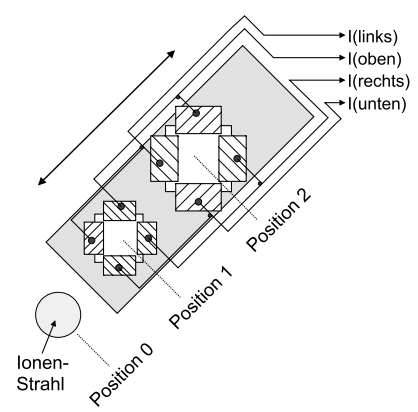

Abbildung 12: Zweifach-Viersektorenblende zur Kontrolle der Strahlposition während der laufenden NRA-Messung. Die Blende kann seitlich in das Strahlrohr eingeschoben werden. Für einen breiten unfokussierten Ionenstrahl erlaubt Pos. 2 ein erstes Auffinden der Strahlposition. Bei der Messung kann der fokussierte Ionenstrahl dann in Pos. 1 gut kontrolliert werden.

Am Ende des $0^{\circ}$-Strahlrohrs des MaRPel-Labors befindet sich der Low-Level NRA-Messplatz. Ein drehbarer Targethalter bietet die Möglichkeit sechs Proben gleichzeitig aufzunehmen. Der Targethalter aus Kupfer ist über eine Kupferlitze thermisch mit einem Kryostaten der Fa. CTI Cryogenics verbunden und lässt sich damit auf Temperaturen von $\mathrm{T} \leq 66 \mathrm{~K}$ herunterkühlen. Zusätzlich besteht die Möglichkeit, den gesamten Targethalter über eine Thermocoax-Heizung aufzuheizen.

Die Aufnahme der Gammaspektren geschieht mittels eines $25 \mathrm{~cm}$-langen NaI-Detektors (Durchmesser: $25 \mathrm{~cm}$ ) mit fünf Photomultipliern. Während der NRA-Messung hat die Probe einen Abstand von $8 \mathrm{~cm}$ zum NaI-Detektor. Wie in Abb. 13 dargestellt, ist der Detektor von aktiven und passiven Komponenten zur Unterdrückung des natürlichen Strahlungsuntergrundes umgeben. Am auffälligsten ist zunächst die Eisenabschirmung aus je $15 \mathrm{~cm}$ starken Eisenplatten. Dieses ,alte Eisen“ stammt von dem 1902 gebauten Panzerkreuzer „Hertog Hendrik“ (s. Anhang E). Im Gegensatz zur modernen Stahlherstellung, bei der Radioisotope bewusst zur Prozesssteuerung in die Schmelze eingebracht werden, ist dieser Stahl sowohl frei von diesen Beimischungen als auch frei von ,anthropogenem“ radioaktiven Fall-Out aus den Kernwaffen-Versuchen und Reaktorkatastrophen. An den vier Wänden und der Decke der Anlage beträgt die Abschirmdicke $30 \mathrm{~cm}$, am Boden $15 \mathrm{~cm}$.

\section{Strahlungsuntergrund und aktive Abschirmung}

Der Strahlungsuntergrund, den es in der Low-Level Messapparatur zu unterdrücken gilt, setzt sich aus der terrestrischen Umgebungsstrahlung und der kosmischen Untergrundstrahlung zusammen.

Die terrestrische Umgebungsstrahlung rührt von den natürlichen Radioisotopen her, die im Gestein und Baumaterial der Experimentierhalle vorkommen. Im Untergrundspektrum (Abb.14) ist sie im Bereich bis $3 \mathrm{MeV}$ der dominierende Faktor. Die stärksten Linien stammen von dem $\beta$-Zerfall des ${ }^{40} \mathrm{~K} z u{ }^{40} \mathrm{Ar}\left(\mathrm{E}_{\gamma}=1.461 \mathrm{MeV}\right)$, das wegen seines großen Anteils von $7 \times 10^{-4}$ am Material der Erdkruste immer auftritt [36], und von ${ }^{232} \mathrm{Th}$, dem Kern mit der längsten Halbwertszeit $\left(\mathrm{T}_{1 / 2}=1.4 \times 10^{10} \mathrm{a}\right)$ der Thorium-Reihe $(\mathrm{A}=4 \mathrm{n})$, die $\mathrm{zu}{ }^{208} \mathrm{~Pb}$ führt $\left(\mathrm{E}_{\gamma}=2.615 \mathrm{MeV}\right)$. 


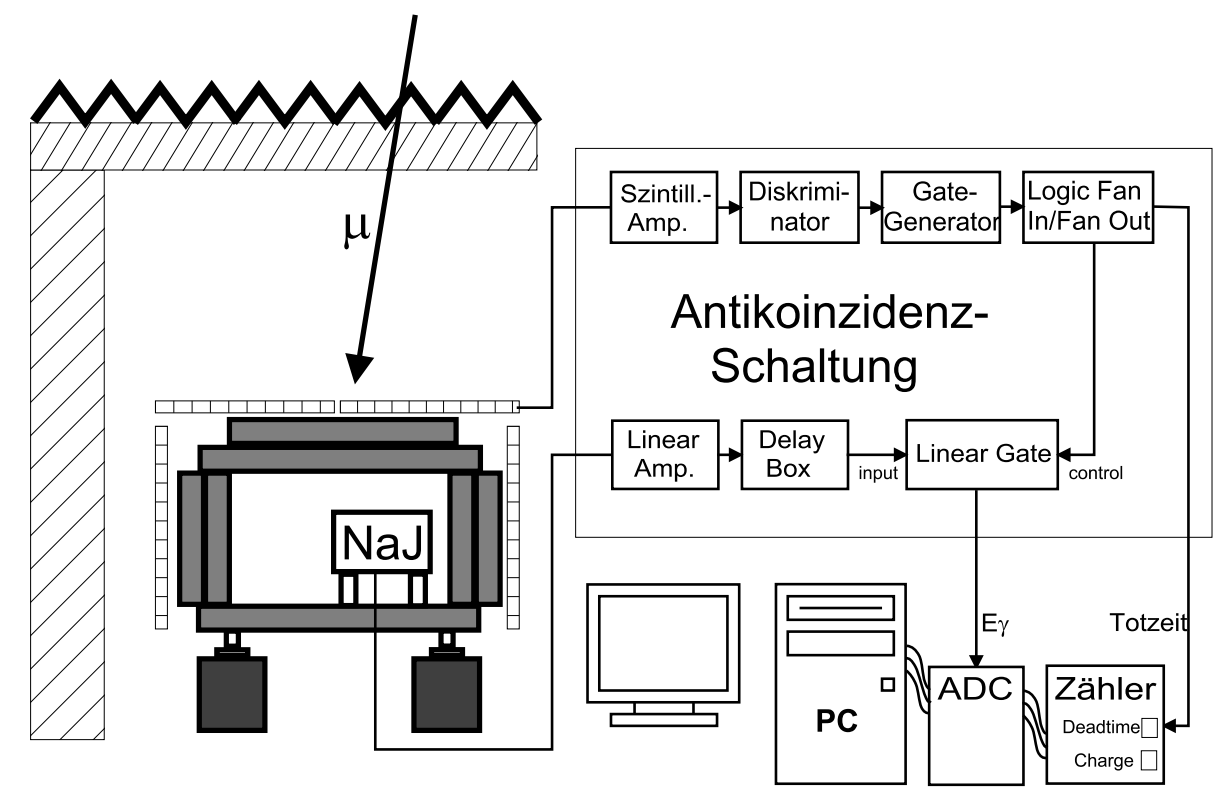

Abbildung 13: Schematischer Aufbau der Antikoinzidenz-Schaltung zur Untergrundunterdrückung am NRA-Messplatz

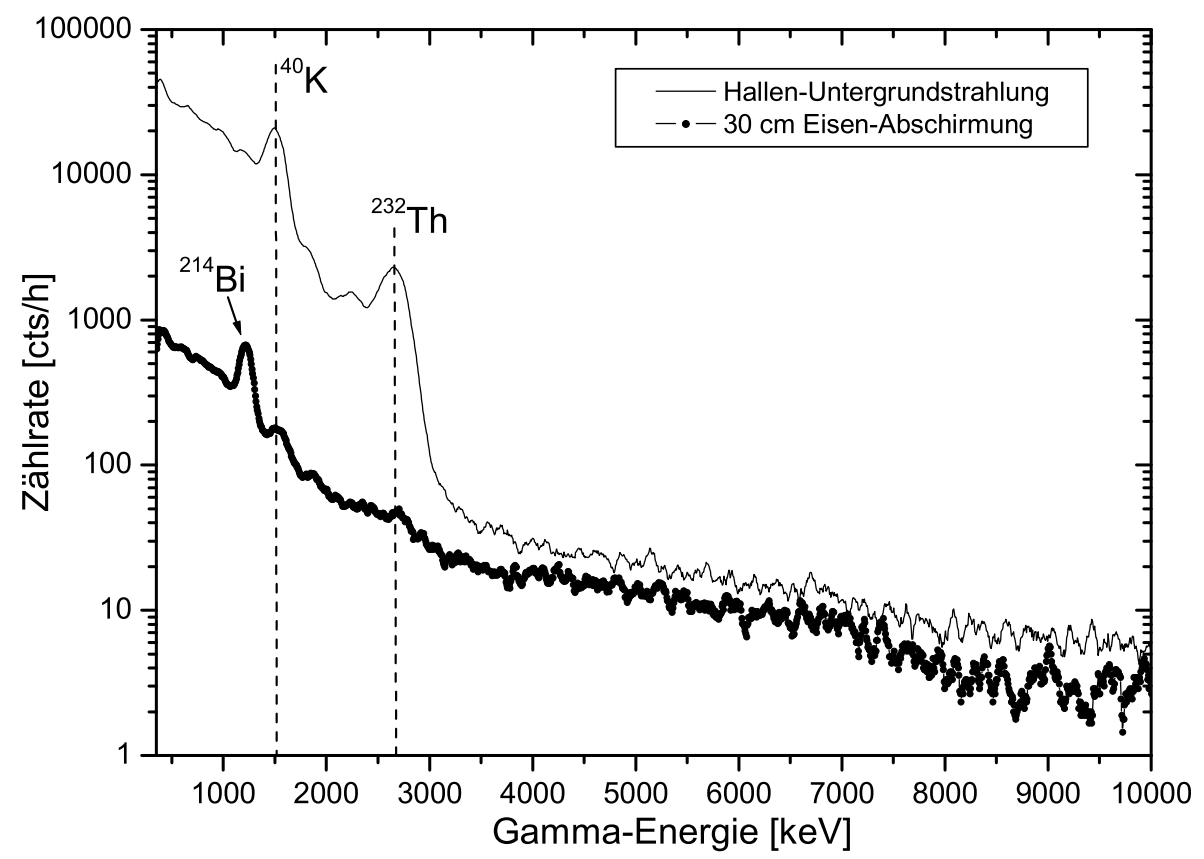

Abbildung 14: Auswirkungen der Maßnahmen zur Untergrund-Unterdrückung am Low-Level NRA-Messplatz 
Im Bereich $\mathrm{E}_{\gamma}>3 \mathrm{MeV}$ überwiegt die kosmische Strahlung. Die kosmische Primärstrahlung besteht hauptsächlich aus Protonen und Alphateilchen und trifft in der oberen Erdatmosphäre auf Sauerstoff- und Stickstoffatome. Dort entstehen durch Spallations- und Teilchenerzeugungsreaktionen vor allem sekundäre Protonen, Neutronen, Pionen und Alphateilchen. Die durch den Zerfall geladener Pionen entstehenden Myonen $\left(\pi^{ \pm} \longrightarrow \mu^{ \pm}\right)$ werden aufgrund ihrer großen Reichweite auch als „harte“ Komponente bezeichnet. Wegen der hohen Teilchenenergien von bis zu $100 \mathrm{GeV}$ dieses Hauptanteils der kosmischen Strahlung ( $\approx 80 \%$ auf Meeresniveau) sind passive Abschirmmaßnahmen hier unwirksam. Der Zerfall ungeladener Pionen $\pi^{0} \longrightarrow 2 \gamma$ führt zu einer elektromagnetischen Kaskade aus Elektronen und Gammastrahlung, deren Wechselwirkung mit Materie über Comptonund Photoeffekt sehr hoch ist (,weiche“ Komponente).

Zusätzlich entstehen durch direkte Prozesse hochenergetischer Pionen mit leichten Kernen hochenergetische Nukleonen. Der Neutronen-Restfluss ist aufgrund der Einfangreaktion ${ }^{127} I(n, \gamma){ }^{128} I$ im Detektorkristall die Ursache für einen erhöhten Strahlungsuntergrund im Bereich 3-7 MeV (vgl. Abb.14).

\subsection{Energieeichung von MaRPel}

Die wichtigsten Größen für den Betrieb eines Ionenbeschleunigers sind die Ionenenergie $\mathrm{E}$ und deren Unschärfe $\Delta \mathrm{E}$. Die Bestimmung dieser beiden Größen mit der Resonanten Kernreaktionsanalyse (NRA) ist Gegenstand des folgenden Abschnitts.

\subsubsection{Energiebreite des Ionenstrahls}

Durch energieverbreiternde Effekte bei der Erzeugung und Beschleunigung der Ionen ist der Ionenstrahl nicht monoenergetisch, sondern er weist eine Energieverteilung auf, die Maschinenbreite $\Delta E_{\text {ion }}$.

Ein erster Beitrag zur Energieverbreiterung ist die Energieunschärfe der Ionenquelle. Für die Alphatross-Ionenquelle gibt der Hersteller NEC den Wert 50-100 eV bei der Erzeugung der positiven Ionen an und zusätzlich etwa den gleichen Beitrag bei der Umladung der Ionen im Rb-Gas. Für die SNICS-Ionenquelle kann eine Energieunschärfe von $\Delta E_{\text {source }} \approx 10-100 \mathrm{eV}[8$ ] angenommen werden.

Ein weiterer Beitrag zur Energieverbreiterung des Ionenstrahls ist gegeben durch die Schwankungen der Terminalspannung („Terminal Ripple“). Bei Tandembeschleunigern verursacht eine Schwankung der Terminalspannung $\Delta U_{\text {rip }}$ für Ionen, die nach der Umladung im Strippergas die Ladung $q$ besitzen eine Energieunschärfe $\Delta E_{\text {rip }}$ von (s. Abs. 2.3 .2

$$
\Delta E_{\text {rip }}=(q+1) \cdot \Delta U_{\text {rip }}
$$

Der Terminalripple $\Delta U_{\text {rip }}$ setzt sich aus zwei Komponenten zusammen. Durch die Ladungsübertragung einzelner Glieder der Pelletronketten auf das Terminal wird eine kurzzeitige Schwankung mit der Dauer von ca. $1 \mathrm{~ms}$ hervorgerufen. Dieser Schwingung ist eine niederfrequente Schwankung mit etwa der zehnfachen Schwingungsdauer überlagert, die wahrscheinlich von mechanischen Schwingungen der Säulenkonstruktion im Tankinnern herrührt (s. Abb.15). Durch die Änderung des Abstandes Terminal-Shell - Corona kommt 
es zu Schwankungen der Terminalkapazität. Nach dem Neuaufbau in Göttingen wurde bei den weiter oben beschriebenen Untersuchungen zur Energiestabilisierung festgestellt, dass der Terminal-Ripple bei gegebener Ionensorte und -energie von vielen Parametern abhängt. So haben beispielsweise die Fokussierung des Ionenstrahls, die Größe des in die Beschleunigerröhre eingeschossenen Strahlstroms und der Strippergasdruck einen großen Einfluß auf die Energieschwankungen des Terminals. Allgemein ließen sich die in [37] angegebenen Werte für den Terminalripple in Heidelberg durch Optimieren der Strahlparameter stets unterschreiten.

Bei Tandem-Beschleunigern spielt der Umladungsprozess im Stripper-Kanal eine ebenso

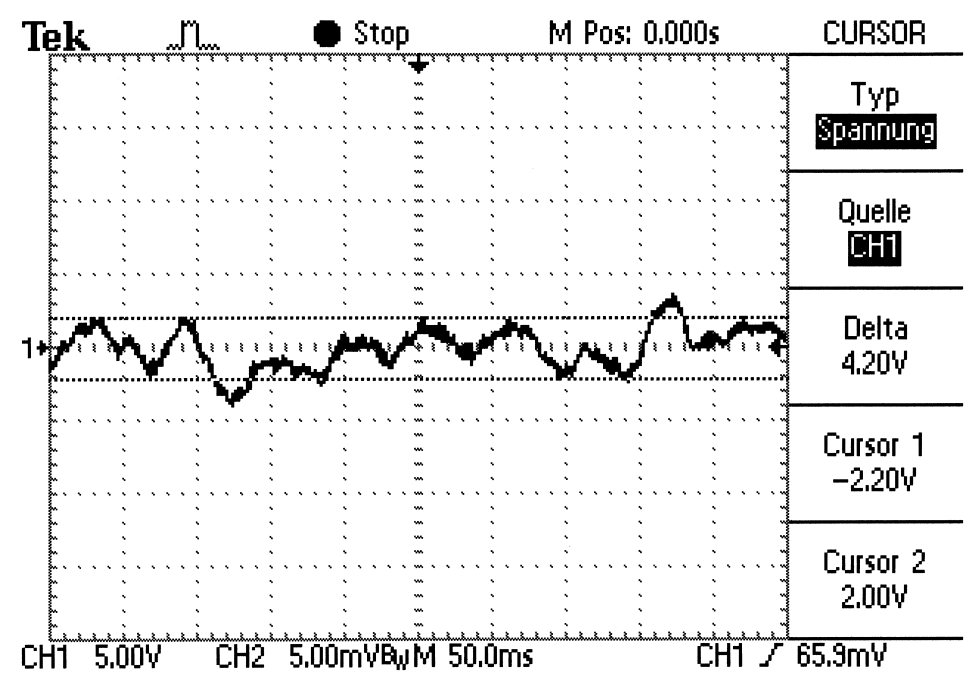

Abbildung 15: Terminalripple, gemessen mit einem Speicheroszillographen. Die hier dargestellte mittlere Schwankung von $\Delta U=4.2 \mathrm{~V}$ muss mit 50 multipliziert werden, um die tatsächliche Schwankung des beschleunigenden Potentials zu erhalten. Mit dem Faktor $K=2$ für den hier verwendeten Protonenstrahl ergibt sich eine Energieaufweitung von $\Delta E=0.42 \mathrm{keV}$ in guter Übereinstimmung mit $\sigma=0.48(4) \mathrm{keV}$ aus Abb. 16 .

wichtige Rolle für die Energieaufweitung des Strahls (vgl. [38, 39]). Dabei muss zunächst zwischen dem Einschuss ionisierter Atome und dem Einschuss von Molekül-Ionen unterschieden werden. Im ersten Fall werden in der Ionenquelle negativ geladene Ionen erzeugt und in den Tank eingeschossen. Im Stripperkanal wird das eingeschossene Ion zunächst neutralisiert und danach werden zusätzliche Elektronen abgestreift, d.h. es ergibt sich folgendes Reaktionsschema:

$$
A^{-} \rightarrow A^{0} \rightarrow A^{+} \rightarrow \ldots \rightarrow A^{n+}
$$

Im Allgemeinen ist für diesen Vorgang die statistische Energieverluststreuung (Straggling) im Strippergas der dominierende Prozess. Durch die Abhängigkeit der Energieverluststreuung vom Ladungszustand des Ions ist eine genaue Berechnung ihres Einflusses schwierig [40, 41]. Für sehr leichte Ionen ( $\mathrm{H}$ und $\mathrm{He}$ ) ist jedoch das Straggling im Strippergas gegenüber der Aufweitung aufgrund des Terminalripple zu vernachlässigen [39]. Werden Molekül-Ionen in den Tank eingeschossen, wie etwa $\mathrm{C}^{15} \mathrm{~N}^{-}$zur Erzeugung eines ${ }^{15} \mathrm{~N}-\mathrm{Strahls}$, so sind mehrere Prozesse im Stripperkanal möglich. Die beiden einfachsten 
Fälle für den Einschuss eines zweiatomigen Moleküls laufen über einen zwei- bzw. dreistufigen Stoßprozess.

Beim neutralen Molekülaufbruch handelt es sich um einen zweistufigen Prozess. Zunächst wird das eingeschossene Molekül beim Passieren des Stripperkanals durch inelastische Stöße mit den Strippergasmolekülen in angeregte Vibrations- und Rotationszustände angehoben. Übersteigt die Anregungsenergie die Bindungsenergie, so dissoziert das Molekül. Gleichzeitig kann es zur Ionisation kommen, jedoch bleibt mindestens ein Fragment unmittelbar nach der Dissoziation neutral. Der Molekülaufbruch findet hier ohne bzw. nur mit geringer Umsetzung der Anregungsenergie in kinetische Energie der Fragmente statt. Durch eine weitere Stoßsionisation entsteht im zweiten Schritt das positiv geladene Fragment.

$$
(A B)^{-} \rightarrow(A B)^{0} \rightarrow A+B \rightarrow A^{+}+B
$$

Die Dissoziationsenergie für das Beispiel des $\mathrm{CN}^{-}$-Moleküls ist mit $E_{\text {diss }}=8.4 \mathrm{eV}$ [42] außerordentlich hoch. Beim Aufbruch des geladenen Moleküls kommen zwei Prozesse in Frage. Die direkte Dissoziation mit Coulomb-Explosion kann in zwei- oder drei Stufen erfolgen und läuft zunächst ähnlich ab, wie der gerade beschriebene Prozess:

$$
(A B)^{-} \rightarrow(A B)^{0} \rightarrow(A B)^{+} \rightarrow(A B)^{2+} \rightarrow A^{+}+B^{+}
$$

Das negativ geladene Molekül wird durch einen ersten inelastischen Stoß neutralisiert und kann dann durch Einfachionisation in zwei Schritten oder auch durch Mehrfachionisation in den doppelt geladenen Ionisationszustand gelangen. Im Unterschied zum vorher beschriebenen Prozess sind hier beide Fragmente beim Molekülaufbruch ionisiert und stoßen sich aufgrund der Coulomb-Wechselwirkung ab. Dabei wird die Coulombenergie in kinetische Energie der Fragmente umgewandelt, es kommt zur sogenannten CoulombExplosion.

Bei beiden Prozessen führt die Moleküldissoziation zu einer Bewegung der Fragmente im Ruhesystem des Moleküls, die der Schwerpunktbewegung überlagert wird. Eine Energieaufweitung des Ionenstrahls im Laborsystem ist die Folge.

Für die Energieaufweitung durch Inhomogenitäten des Magnetfeldes im 90-Magneten gibt der Hersteller des Magneten

$$
\frac{\Delta E}{E}=2 \cdot 10^{-4}
$$

an [43. Damit ergibt sich eine Energieunschärfe von $\Delta \mathrm{E}_{\text {mag }}(\mathrm{p})=0.2 \mathrm{keV}$ für $1 \mathrm{MeV}$ Protonen und $\Delta \mathrm{E}_{\text {mag }}\left({ }^{15} \mathrm{~N}\right)=1.3 \mathrm{keV}$ für $6.385 \mathrm{MeV}{ }^{15} \mathrm{~N}$-Ionen.

Insgesamt erfährt der Ionenstrahl von der Quelle zum Target eine Energieaufweitung von

$$
\Delta E_{\text {ion }}=\sqrt{\Delta E_{\text {source }}^{2}+\Delta E_{\text {ripple }}^{2}+\Delta E_{\text {Mol }}^{2}+\Delta E_{\text {stragg }}^{2}+\Delta E_{\text {Mag }}^{2}}
$$

Setzt man die Größen für einen Protonen- respektive ${ }^{15} \mathrm{~N}-S t r a h l$ ein, so erhält man die in Tabelle 1 angebenen theoretischen Werte. Ebenfalls aufgeführt sind experimentell ermittelte Werte für die Energieaufweitung. Für den Protonenstrahl wurde der Wert über die Bestrahlung einer massiven Al-Probe im Bereich zweier schmaler Kernresonanzen gemessen. Abbildung 16 zeigt das NRA-Spektrum einer reinen Al-Probe. Das Spektrum wurde 


\begin{tabular}{c|c|c|c|c} 
Ion & $\mathrm{E}_{\text {ion }}[\mathrm{keV}]$ & $\Delta E_{\text {Mol }}[\mathrm{keV}]$ & $\Delta E_{\text {ion }}($ theor.) $[\mathrm{keV}]$ & $\Delta E_{\text {ion }}($ exp. $)[\mathrm{keV}]$ \\
\hline \hline $\mathrm{p}$ & 1000 & 0 & 1.5 & $0.48(3)$ \\
\hline${ }^{15} \mathrm{~N}$ & 6385 & 2.2 & 5 & $\approx 7(1)$ \\
\hline
\end{tabular}

Tabelle 1: Energieaufweitung $\Delta E_{\text {ion }}$ für einen Protonen- bzw. ${ }^{15} N$-Strahl. $\Delta E_{\text {ion }}$ für den ${ }^{15} N$ Strahl wurde aus der gemittelten Varianz schmaler H-Oberflächenpeaks abgeschätzt.

mit der minimal möglichen Schrittweite der Amselsteuerung von $\Delta \mathrm{E}=0.102 \mathrm{keV}$ vermessen. In der linken Auftragung ist der Energiebereich $\mathrm{E}_{p}=985-1035 \mathrm{keV}$ dargestellt, in dem zwei schmale Kernresonanzen des Aluminiums liegen. Die ${ }^{27} \mathrm{Al}(\mathrm{p}, \gamma){ }^{28} \mathrm{Si}-\mathrm{Kernreaktionen}$ haben die Resonanzenergien $\mathrm{E}_{R 1}=992 \mathrm{keV}$ und $\mathrm{E}_{R 2}=1024 \mathrm{keV}$ mit den Resonanzbreiten $\Gamma_{1}=0.05 \mathrm{keV}$ [44, bzw. $\Gamma_{2} \leq 0.24 \mathrm{keV}$ [45. Die rechte Abbildung ist eine Vergrößerung der linken im Energiebereich $\mathrm{E}_{p}=990-995 \mathrm{keV}$. Eine Anpassung einer Fehlerfunktion an die Messdaten liefert den Wert $\sigma=0.48(4) \mathrm{keV}$ für die Varianz. Die Hauptbeiträge zu diesem Wert sind die Energiebreite des Ionenstrahls $\Delta \mathrm{E}$ und die Resonanzbreite der Kernreaktion $\Gamma_{1}$. In der Näherung durch Gaußfunktionen ergibt sich für die Energiebreite des Ionenstrahls $\Delta \mathrm{E}=\sqrt{\sigma^{2}-\Gamma_{1}^{2}}=0.48(3)$.
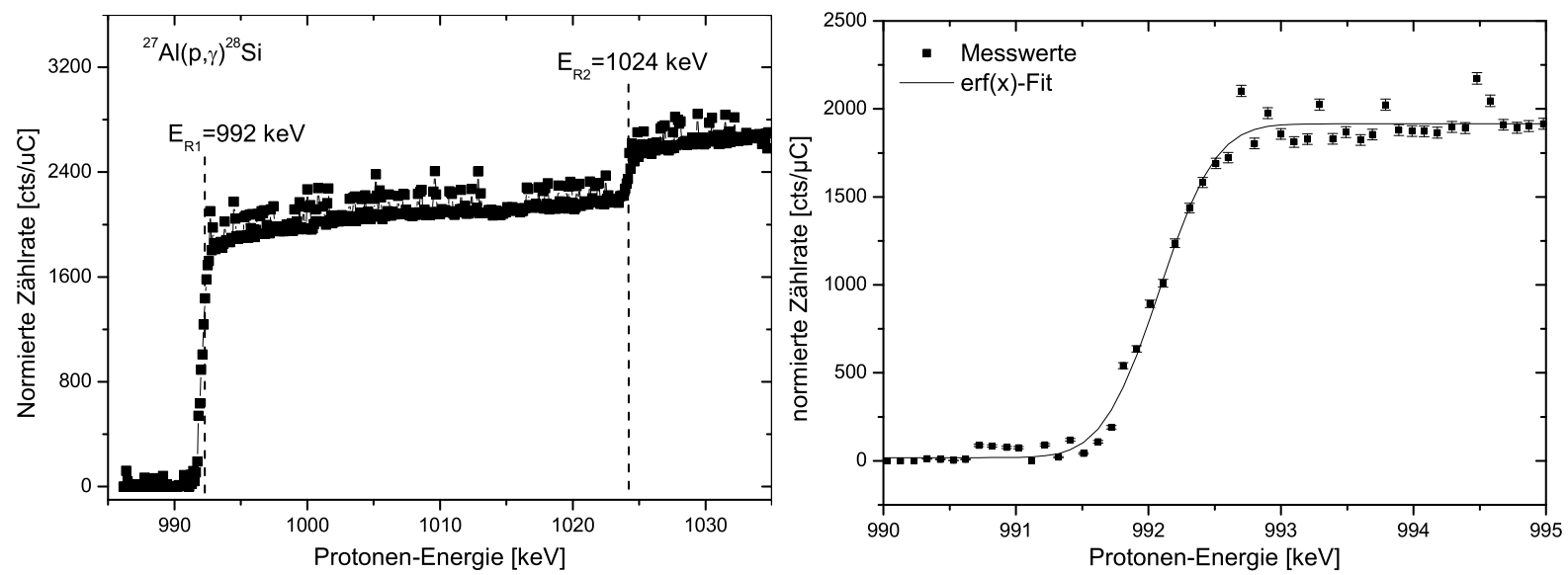

Abbildung 16: Links: NRA-Spektrum für die Bestrahlung einer massiven Al-Probe mit Protonen im Energiebereich $E_{p}=887-1035 \mathrm{keV}$. Die resonanten ${ }^{27} \mathrm{Al}(p, \gamma)^{28}$ Si-Kernreaktionen sind als Stufen bei den Resonanzenergien $E_{R 1}=992 \mathrm{keV}$ und $E_{R 2}=1024 \mathrm{keV}$ zu sehen. Die Kernresonanzen besitzen eine sehr kleine Resonanzbreite von $\Gamma_{1}=0.05 \mathrm{keV}$ [44], bzw. $\Gamma_{2} \leq 0.24 \mathrm{keV}$ [45]. Rechts: Vergrößerung im Energiebereich $E_{p}=890-995 \mathrm{keV}$. Aus der angefitteten Fehlerfunktion ergibt sich eine Varianz von $\sigma=0.48(4) \mathrm{keV}$. Durch die kleine Resonanzbreite der ${ }^{27} \mathrm{Al}(p, \gamma){ }^{28}$ Si-Kernreaktion ist $\sigma$ eine obere Schranke für die Energiebreite des Protonenstrahls. 


\subsubsection{Energieeichung des $90^{\circ}$-Magneten}

Die Schwierigkeit der Energieeichung ergibt sich aus zwei zunächst nur näherungsweise bekannten Werten. Zum einen ist die Größe des beschleunigenden Potenzials V nur sehr ungenau bekannt. Das Potenzial V wird mittels eines Rotationsvoltmeters (GVM) bestimmt (s. Abs. 2.1.3), welches beim Transport von Heidelberg nach Göttingen zerlegt werden musste und in Göttingen neu montiert wurde. Die GVM-Anzeige am TPS-System muss mit einem bekannten Potenzial an der Terminal-Shell durch Einstellen des TerminalPotential-Stabilizer Systems abgeglichen werden.

Die zweite mit einer gewissen Ungenauigkeit behaftete Größe ist der Krümmungsradius des $90^{\circ}$-Magneten. Der Umzug von Heidelberg nach Göttingen machte eine Neueichung des $90^{\circ}$-Magneten notwendig, da die Magnetkammer, wie bereits in Abs. 2.1 erwähnt, aufgrund der räumlichen Gegebenheiten in Göttingen um $180^{\circ}$ gedreht aufgebaut werden musste. Während der Strahl im Heidelberger Labor durch den 90-Magneten -in Strahlrichtung gesehen- nach links abgelenkt wurde, wird der Ionenstrahl im Göttinger MaRPel-Labor nach rechts abgelenkt. Durch diese Spiegelung der Kammer konnte sich der Radius innerhalb eines kleinen Bereichs geändert haben.

Im folgenden soll das Verfahren beschrieben werden, mit dem die Energieeichung durchgeführt wurde. Durch die GVM-Anzeige ist das beschleunigende Potenzial ungefähr bekannt. Dann lässt sich nach

$$
E_{G V M}=\frac{q}{K} V \quad \text { mit } \quad K=\frac{q}{q+\frac{m_{A}}{m_{B}+m_{A}}}
$$

die Energie des Ionenstrahls $E_{G V M}$ berechnen, die sich gemäß der GVM-Anzeige von $\mathrm{V}$ ergeben würde (q: Ionenladung nach dem Umladungsprozess im Strippergas-Kanal, $m_{A}$ : Ionenmasse nach Passieren des Strippergas-Kanals, $m_{A}+m_{B}$ : Gesamtmasse des eingeschossenen Moleküls). Für einen (einfach geladenen) Protonenstrahl, der als $\mathrm{H}^{-}$-Ion eingeschossen wird, gilt: $E_{G V M}=2 \cdot V$.

Zunächst wurde das Magnetfeld B, welches mittels der Hall-Sonde innerhalb der Polschuhe sehr genau $(\Delta B \leq 0.1 m T)$ bestimmt werden kann, gemäß der Formel (vgl. Anhang A)

$$
B=\frac{m_{0} c}{q R} \cdot \sqrt{\left(\frac{E+m_{0} c^{2}}{m_{0} c^{2}}\right)^{2}-1}
$$

unter Verwendung des Heidelberger Krümmungsradius $\mathrm{R}=844 \mathrm{~mm}$ für die nach Gl. 11 bestimmte Strahlenergie eingestellt. Durch kleine Änderungen des Potenzials V wurde dann der Ionenstrahl auf den Faraday-Cup am Ausgang des 90-Magneten (vgl. Abb. 1) fokussiert. War ein Maximum des Strahlstroms im Faraday-Cup erreicht, so wurde der Wert des beschleunigenden Potenzials V am Rotationsvoltmeter abgelesen. Diese Prozedur wurde für verschiedene Einstellungen des Potenzials V und des 90-Magnetfelds B wiederholt. Damit war eine erste Zuordnung der Energie $\mathrm{E}_{G V M}$ zum Magnetfeld B gegeben.

Die tatsächliche Energie der Ionen war damit allerdings noch nicht festgelegt, da der Krümmungsradius $\mathrm{R}$ des Magneten nicht exakt bekannt war. Für eine exakte Energieeichung wurden vier ${ }^{15} \mathrm{~N}-$ Kernresonanzen einer lasernitrierten TaN-Probe vermessen [46]. 


\begin{tabular}{c|c|c|c|c} 
Reaktion & $\mathrm{E}_{R}[\mathrm{keV}]$ & $\Gamma[\mathrm{keV}]$ & $\sigma[\mathrm{mbarn}]$ & Ref. \\
\hline \hline${ }^{15} N(p, \alpha \gamma){ }^{12} C$ & 898 & 2.2 & 800 & {$[47]$} \\
\hline${ }^{15} N(p, \alpha \gamma){ }^{12} C$ & 1210 & 22.5 & 425 & {$[48]$} \\
\hline${ }^{15} N(p, \alpha \gamma){ }^{12} C$ & 1640 & 68 & 340 & {$[49]$} \\
\hline${ }^{15} N(p, \alpha \gamma){ }^{12} C$ & 1979 & 23 & 35 & {$[48]$} \\
\hline
\end{tabular}

Tabelle 2: Verwendete ${ }^{15} \mathrm{~N}$-Kernresonanzen, $E_{R}$ : Resonanzenergie, $\Gamma$ : Resonanzbreite, $\sigma$ : Wirkungsquerschnitt

Das schwere Element Ta wurde als Substrat verwendet, weil es keine eigenen Resonanzen im untersuchten Energiebereich aufweist. Die Daten dieser Kernresonanzen sind in Tab. 2 mit den entsprechenden Referenzen angegeben. Die zugehörigen Resonanzkurven sind in Abb. 17 dargestellt. Die Strahlenergie wurde dabei mittels der Amsel-Deflektoren leicht variiert (vgl. Abs. 2.3.3), um das gesamte ${ }^{15} \mathrm{~N}$-Tiefenprofil aufzunehmen. Die vier
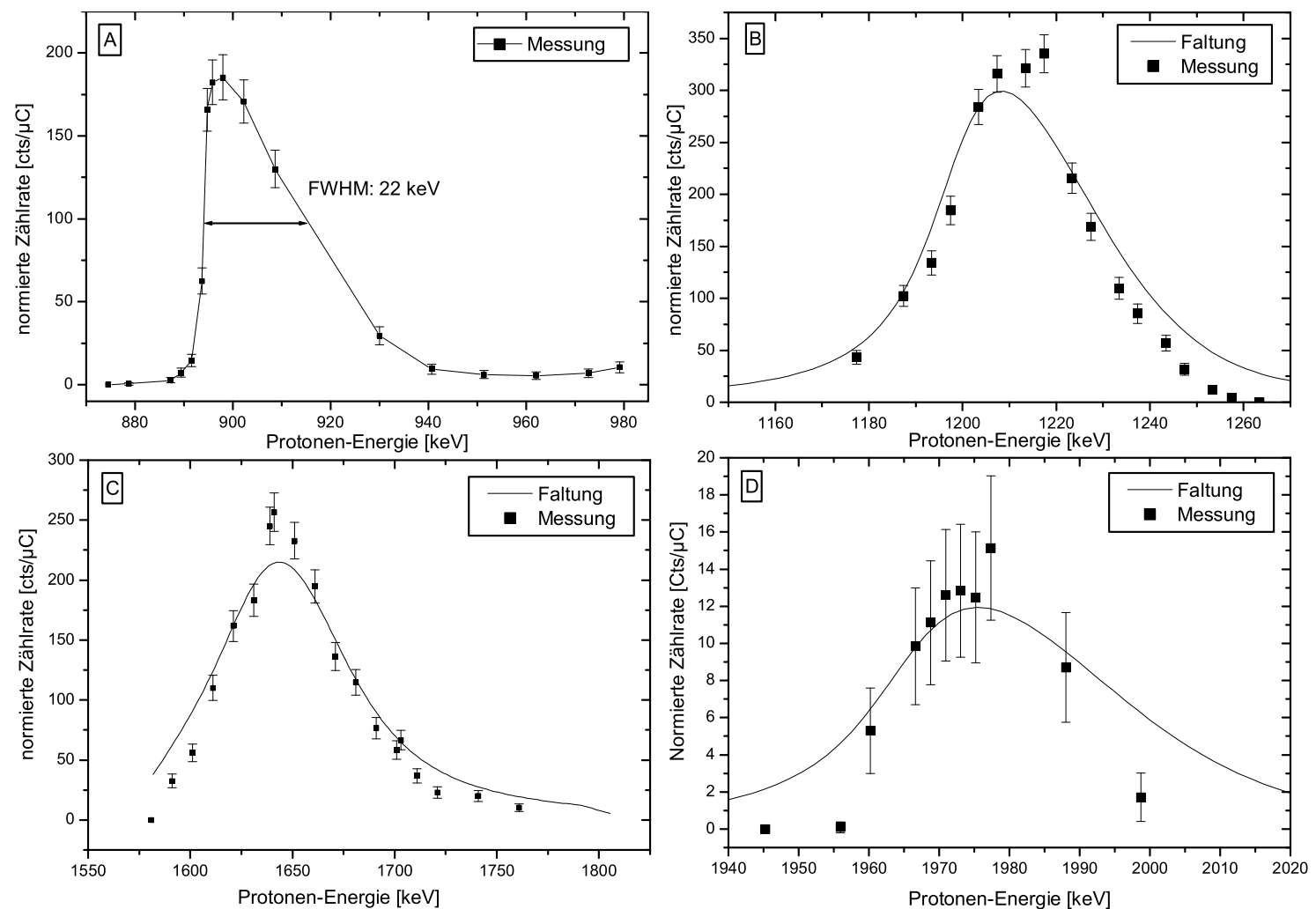

Abbildung 17: Resonanzkurven der ${ }^{15} N(p, \alpha \gamma){ }^{12} C$-Kernresonanz bei den Resonanzenergien (A) $E_{R 1}=898 \mathrm{keV},(B) E_{R 2}=1210 \mathrm{keV},(C) E_{R 3}=1640 \mathrm{keV}$ und $(D) E_{R 4}=1979 \mathrm{keV}$. Zusätzlich zu den Messwerten (Quadrate) sind die Faltungsintegrale aus Spektrum (A) mit einer Breit-WignerForm eingezeichnet.

in Abb. 17 gezeigten Resonanzen unterscheiden sich sehr stark in ihrer Form. Die Unterschiede rühren dabei hauptsächlich von den unterschiedlichen Breiten der Kernreaktionen her (vgl. Tab. 2). Während die schmale Resonanz bei $E_{R 1}=898 \mathrm{keV}$ die Tiefenverteilung des Stickstoffs in der Probe wiedergibt (Spektrum (A) in Abb. 17), sind die übrigen Spek- 
tren durch die größere Resonanzbreite ,verschmiert“.

Die Breite der Stickstoff-Verteilung liegt mit $22 \mathrm{keV}$ (FWHM) in der Größenordnung der Resonanzbreiten der Kernreaktionen $\mathrm{E}_{R 2}=1210 \mathrm{keV}$ und $\mathrm{E}_{R 4}=1979 \mathrm{keV}$ und die Resonanz bei $\mathrm{E}_{R 3}=1640 \mathrm{keV}$ besitzt sogar die dreifache Energiebreite der Stickstoff-Tiefenverteilung. Um die exakte Lage der Resonanzenergie aus den gezeigten Spektren zu erhalten, wurde an die Spektren (B), (C) und (D) eine Faltung aus Spektrum (A) und einer Breit-WignerForm mit der Breite der jeweiligen Kernresonanz angepaßt. Die so erhaltenen Faltungsintegrale sind ebenfalls in Abb. 17 dargestellt.

Bei den vier nun genau definierten Energien des Protonenstrahls konnte dann ein Abgleich der GVM-Anzeige durchgeführt werden. Damit war das beschleunigende Potenzial V exakt definiert. Außerdem konnten den vorher aufgenommenen Wertepaaren von Potenzial $\mathrm{V}$ und Magnetfeld B die entsprechenden Ionenenergien zugeordnet werden, so dass diese Werte in eine Auftragung des Magnetfeldes B über der Ionenenergie E mit den vier aus den Kernresonanzen ermittelten Energiewerten mit aufgenommen werden konnten. Aus der

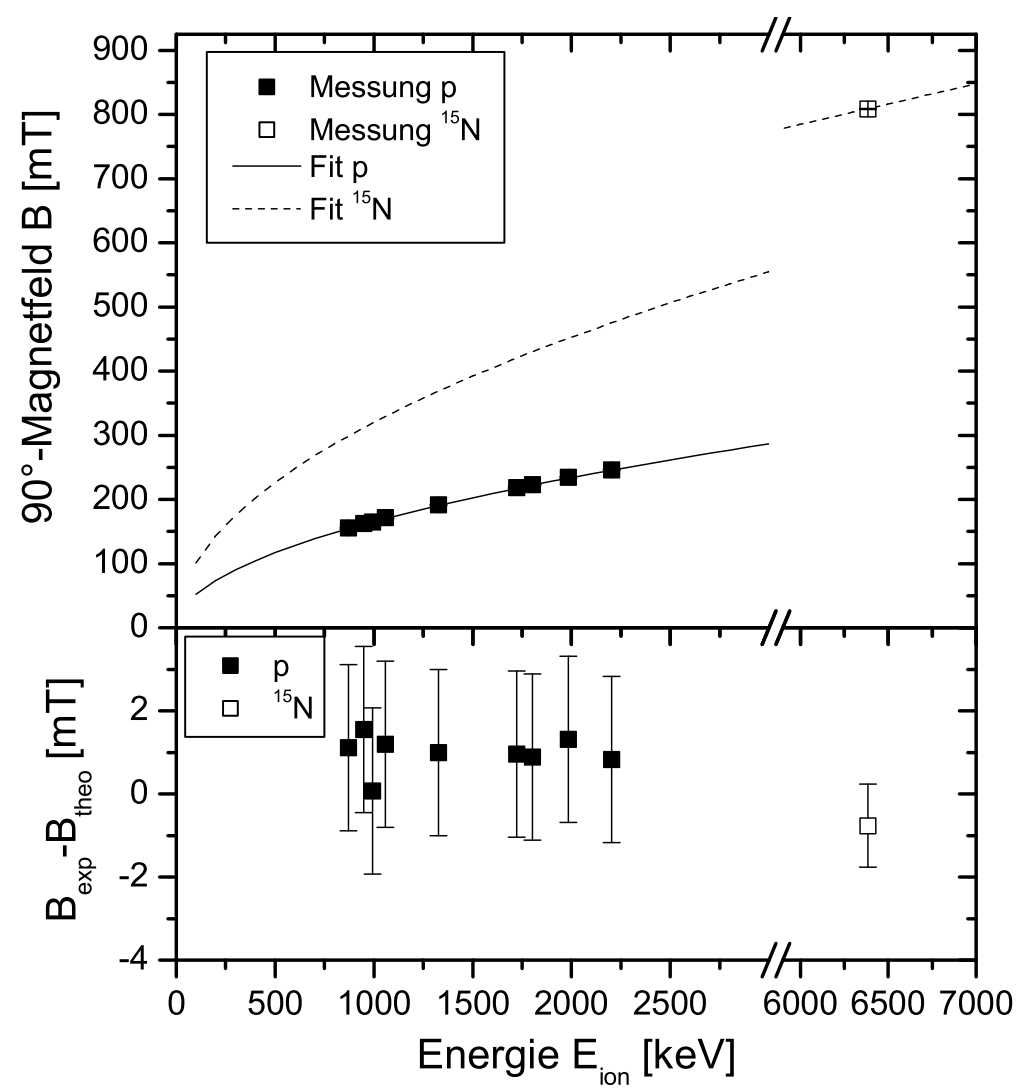

Abbildung 18: Oben: Magnetfeld B zur Ablenkung eines Protonen- bzw. ${ }^{15} \mathrm{~N}$-Strahls um $90^{\circ}$ in Abhängigkeit von der Ionenenergie. Zusätzlich zu den experimentellen Werten (Punkte) ist die Anpassung mittels Gl. 12 mit dem Krümmungsradius $R$ als freiem Parameter dargestellt. Unten: Abweichung der experimentellen Werte von der Anpassung mit $R=0.871(1) \mathrm{m}$

Auftragung im oberen Teil von Abb. 18 ergibt sich der tatsächliche Krümmungsradius R aus der Anpassung mittels Gl. $12 \mathrm{zu} \mathrm{R}=871(1) \mathrm{mm}$. Auch der experimentelle Wert für das Magnetfeld für die ${ }^{1} \mathrm{H}\left({ }^{15} \mathrm{~N}, \alpha \gamma\right){ }^{12} \mathrm{C}$-Kernreaktion bei der Resonanzenergie $\mathrm{E}_{R}=6385 \mathrm{keV}$, die in der vorliegenden Arbeit zum Wasserstoff-Nachweis genutzt wurde, ist im oberen Teil von Abb. 18 mit eingezeichnet. Über die Güte der Anpassung gibt der untere Teil 
von Abb. 18 Auskunft. Hier sind die Abweichungen der Messwerte von der angepassten B(E)-Funktion angegeben. Die Fehler der Anpassung liegen im Promille-Bereich.

\subsubsection{Amsel-Energie-Scanning-System}

Zur Messung einer Resonanzkurve mittels NRA muss die Strahlenergie systematisch in kleinen Schritten variiert werden. Da die Strahlenergie durch das Magnetfeld des $90^{\circ}$ Magneten bestimmt wird, müsste für NRA-Messungen das Magnetfeld in möglichst kleinen Schritten erhöht werden. In der Handhabung ergibt sich dabei eine große Schwierigkeit aus der Hysterese des Magneten, die kleine Änderungen des Feldes mühsam macht. Das in Kapitel 2.1.3 beschriebene TPS-System kann nach einer Idee von Amsel et al. [50] zur hysteresefreien Variation der Strahlenergie genutzt werden. Abbildung 19 zeigt den schematischen Aufbau des Amsel-Energie-Scanning-Systems. Symmetrisch um den Einund Ausgang des $90^{\circ}$-Magneten befinden sich Ablenkplattenpaare (Deflektor 1 und Deflektor 2). Die durchgezogene Linie stellt den idealen Strahlverlauf bei fester Ionenenergie und festem Magnetfeld dar. In diesem Fall liegt an den Ablenkplatten keine Spannung an. Wird an die Deflektorplatten eine Spannung derart angelegt, dass die jeweils in Strahl-

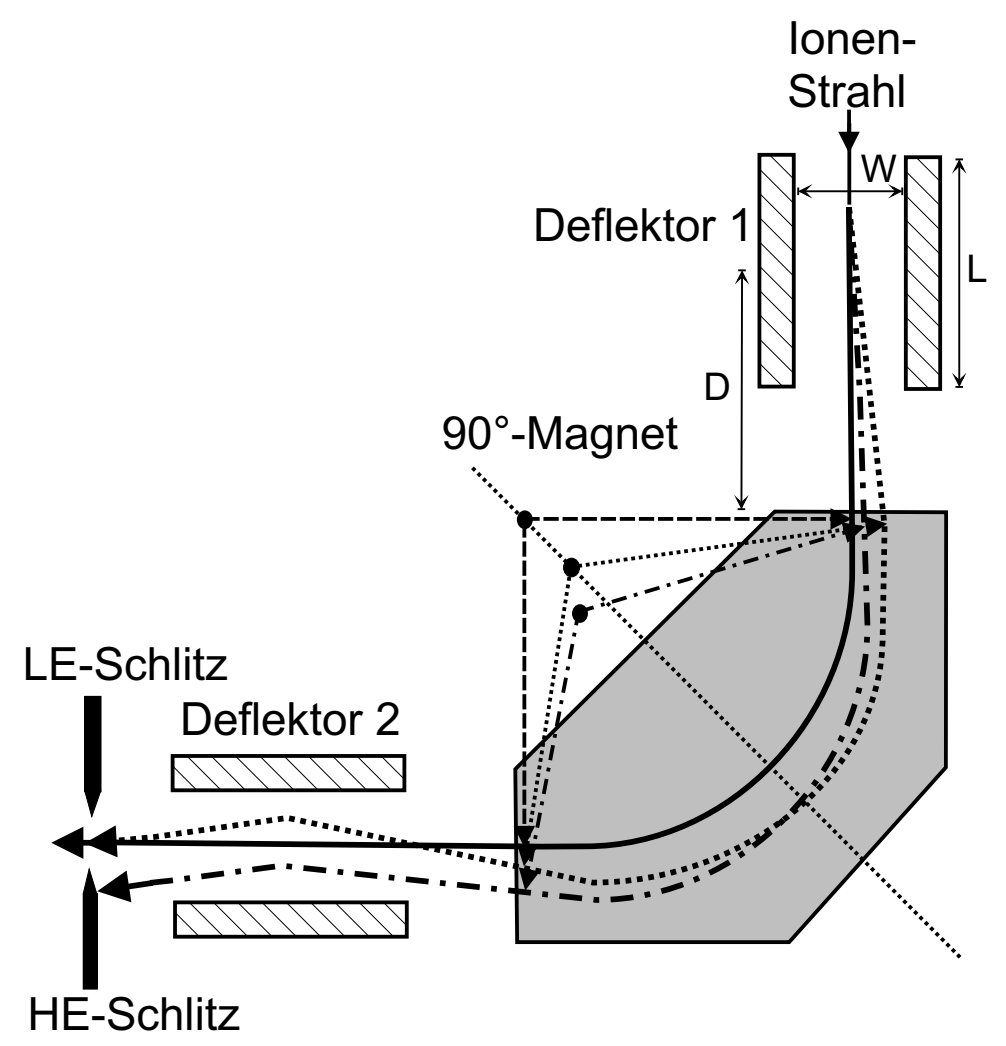

Abbildung 19: Strahlverlauf in Amsel-Deflektoren und 90-Magnet

richtung linke Platte auf negativem Potenzial liegt, so werden die Ionen zunächst der strich-punktiert eingezeichneten Bahn folgen. Diese Bahn verläuft im allgemeinen unsymmetrisch durch den Magneten, der Radiuspunkt liegt nicht auf der Winkelhalbierenden. Wie in der Skizze zu erkennen ist, führt diese Ionenbahn zu einem höheren Schlitzstrom 


\begin{tabular}{c|c|c|c|c} 
Ion & Ladung & $K$ & $G_{0}$ (theor. $)$ & $G_{0}($ exp. $)$ \\
\hline \hline Protonen & 1 & 0.5 & 5.24 & $5.15(5)$ \\
\hline${ }^{15} \mathrm{~N}^{2+}$ & 2 & 0.783 & 8.21 & $7.97(8)$ \\
\hline
\end{tabular}

Tabelle 3: Gemessene und berechnete Verstärkungsfaktoren für Protonen- und ${ }^{15} \mathrm{~N}$-Strahl

auf dem Hochenergie-Schlitz. Das TPS-System erhält folglich das Signal zur Verminderung der Strahlenergie und es wird sich die punktiert eingezeichnete Ionenbahn einstellen, die wieder symmetrisch durch den $90^{\circ}$-Magneten verläuft.

Diese Rückkopplung macht es möglich, die Energie des Ionenstrahls bei festem Magnetfeld des $90^{\circ}$-Magneten durch das Anlegen einer Hochspannung $(0 \leq \mathrm{U} \leq 20 \mathrm{kV})$ an den Deflektorplatten hysteresefrei und in kleinen Schritten zu verändern. Eine quantitative Bestimmung der Energieverschiebung folgt nach [32] aus den ebenfalls in Abb. 19 eingezeichneten Geometriefaktoren. Für den Fall der Ablenkung des Ionenstrahls gilt in nullter Näherung eine lineare Beziehung zwischen der Veränderung des beschleunigenden Potenzials $\Delta V$ und der Spannung $U$ an den Deflektoren (vgl. Anhang B):

$$
\Delta V=G_{0} \cdot U
$$

Die Proportionalitätskonstante $\mathrm{G}_{0}$ wird Verstärkungsfaktor genannt. Mit der Länge der Deflektoren L, deren Plattenabstand W, dem Abstand D der Plattenmitte zu den Polschuhen und dem Krümmungsradius $\mathrm{R}$ des $90^{\circ}$-Magneten gilt:

$$
G_{0}=\frac{L}{W} K\left(1-\frac{D}{R}\right)
$$

Der Faktor $K$ beinhaltet sowohl die Ladungsabhängigkeit der Ionenenergie bei Tandembeschleunigern, als auch den Massenquotienten für den Molekülaufbruch:

$$
K=\frac{q}{q+\frac{m_{B}}{m_{A}+m_{B}}}
$$

Dabei bezeichnet q den Ladungszustand der Ionen auf der zweiten Beschleunigungsstrecke nach dem Passieren des Stripper-Kanals, $m_{B}$ deren Masse und $\left(\mathrm{m}_{A}+\mathrm{m}_{B}\right)$ die Masse des eingeschossenen Moleküls. Mit den Werten $\mathrm{W}=23 \mathrm{~mm}, \mathrm{~L}=300 \mathrm{~mm}, \mathrm{D}=170 \mathrm{~mm}$ und $\mathrm{R}=871 \mathrm{~mm}$ für unseren Aufbau ergeben sich die in Tabelle 3 angegebenen Verstärkungsfaktoren für einen Protonen- respektive ${ }^{15} \mathrm{~N}-\mathrm{Strahl}$.

\section{Experimentelle Bestimmung des Verstärkungsfaktors}

Der Wert für den Verstärkungsfaktor beim Protonenstrahl wurde direkt aus der Messung einiger dicht liegender Al-Resonanzen im Energiebereich 730-780 keV gewonnen. In Abb. 20 ist die normierte Gamma-Zählrate über der Deflektor-Spannung U aufgetragen. Man sieht jeweils bei den mit Zahlen gekennzeichneten Werten für die Deflektor-Spannung $\left(U_{1}\right.$ bis $\left.\mathrm{U}_{5}\right)$ einen starken Anstieg der Zählrate, der auf Resonanzen der Kernreaktion ${ }^{27} \mathrm{Al}(p, \gamma){ }^{28} \mathrm{Si}$ zurückzuführen ist. 


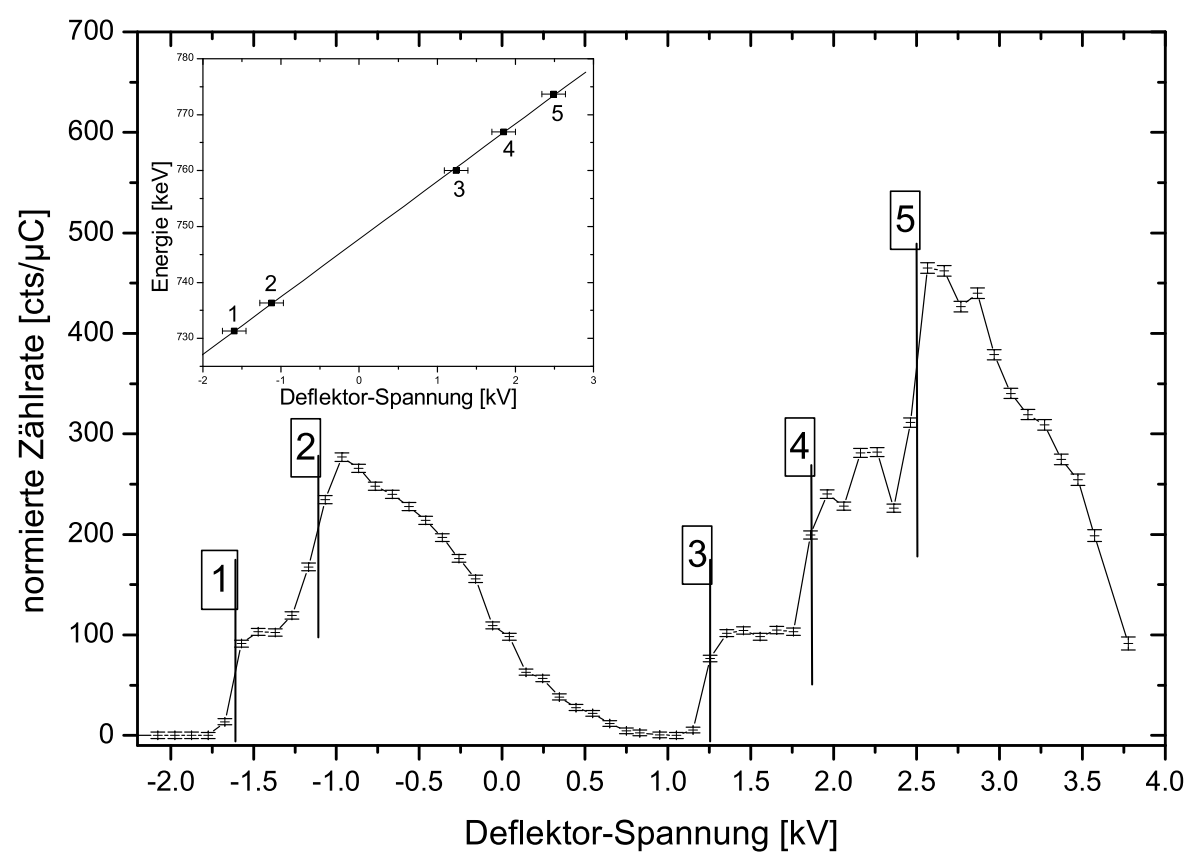

Abbildung 20: NRA-Spektrum einer $100 \mathrm{~nm}$ Al-Schicht auf Cu. Die Al-Resonanzen bei den Energien 1) $\left.E_{R 1}=731.3 \mathrm{keV}, 2\right) E_{R 2}=736.3 \mathrm{keV}$, 3) $E_{R 3}=760 \mathrm{keV}$, 4) $E_{R 4}=766.9 \mathrm{keV}$ und 5) $E_{R 5}=773.7 \mathrm{keV}$ [51] sind wegen der „Dicke“ der Al-Schicht als Stufen zu erkennen. Der Einsatz zeigt eine Auftragung der fünf Resonanzenergien über der zugehörigen Deflektor-Spannung U, die jeweils am Punkt der halben Stufenhöhe abgelesen wurde. Die Steigung der Geraden entspricht dem doppelten experimentellen Verstärkungsfaktor $2 \cdot G_{0}($ exp.)

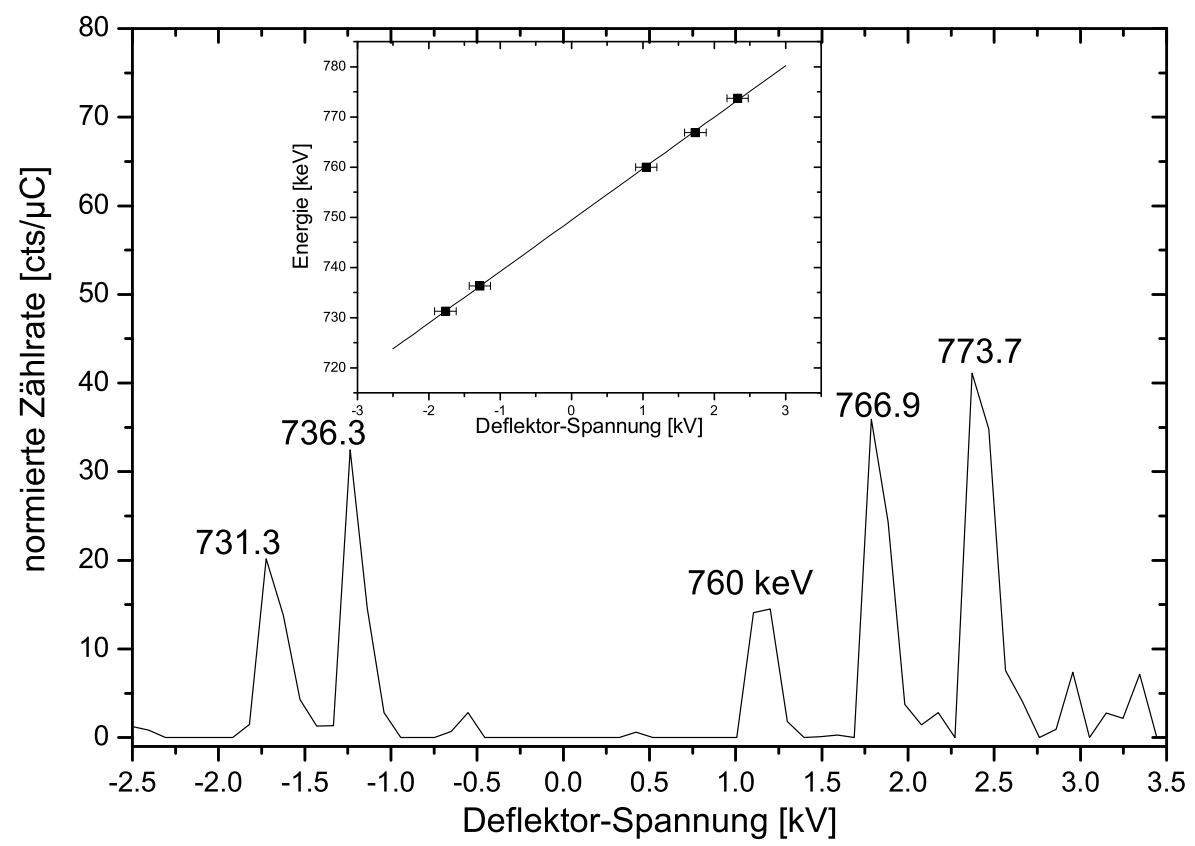

Abbildung 21: NRA-Spektrum einer nur $10 \mathrm{~nm}$ dicken Al-Schicht auf Tantal. Wieder sind die Resonanzen aus Abb. 20 zu sehen, die jetzt jedoch wesentlich schmaler sind. Der Einsatz zeigt eine Auftragung der fünf Resonanzenergien über der zugehörigen Deflektor-Spannung U. Die Steigung der Geraden entspricht exakt dem mittels Abb. 20 ermittelten Wert. 
Mit der Kenntnis der exakten Resonanzenergien (Literaturwerte aus [51]) kann dann durch eine Auftragung der Protonenenergie über der Deflektorspannung (s. Einsatz in Abb. 20p der Verstärkungsfaktor $G_{0}$ (exp.) experimentell ermittelt werden. Die Werte der Deflektorspannung wurden dabei jeweils am Punkt der halben Stufenhöhe abgelesen. Die gezeigte lineare Regression ergibt unter Berücksichtigung von $\Delta E=2 \cdot \Delta V$ (s. Gl. 11) den Wert $G_{0}(\exp )=.5.15(5)$. Dieser Wert ergibt sich ebenfalls bei Verwendung einer $10 \mathrm{~nm}$ dicken Al-Schicht auf einem Tantal-Substrat (Abb. 21), so dass mögliche Fehler in der Bestimmung der zur Resonanz gehörenden Deflektorspannung U, die von der Dicke der Al-Schicht herrühren, in Abb. 20 zu vernachlässigen sind.

Die Bestimmung des Verstärkungsfaktors für den ${ }^{15} \mathrm{~N}$-Strahl muss dagegen mittels der GVM-Anzeige erfolgen, da die energetisch gesehen nächste ${ }^{1} H\left({ }^{15} N, \alpha \gamma\right){ }^{12} C$-Kernresonanz mit $E_{R}=13.4 \mathrm{MeV}$ mit MaRPel nicht erreicht werden kann. Die GVM-Anzeige bietet die Möglichkeit, die Größe des beschleunigenden Potenzials V mit einer Genauigkeit von ca. $\Delta \mathrm{V}=2 \mathrm{kV}$ zu bestimmen. Damit können die Veränderungen der Hochspannung $\mathrm{V}$ durch das Anlegen der Deflektor-Spannung U direkt abgelesen werden. Abb. 22 zeigt eine Auftragung der Hochspannungsänderung $\Delta \mathrm{V}$ als Funktion der Deflektor-Spannung U.

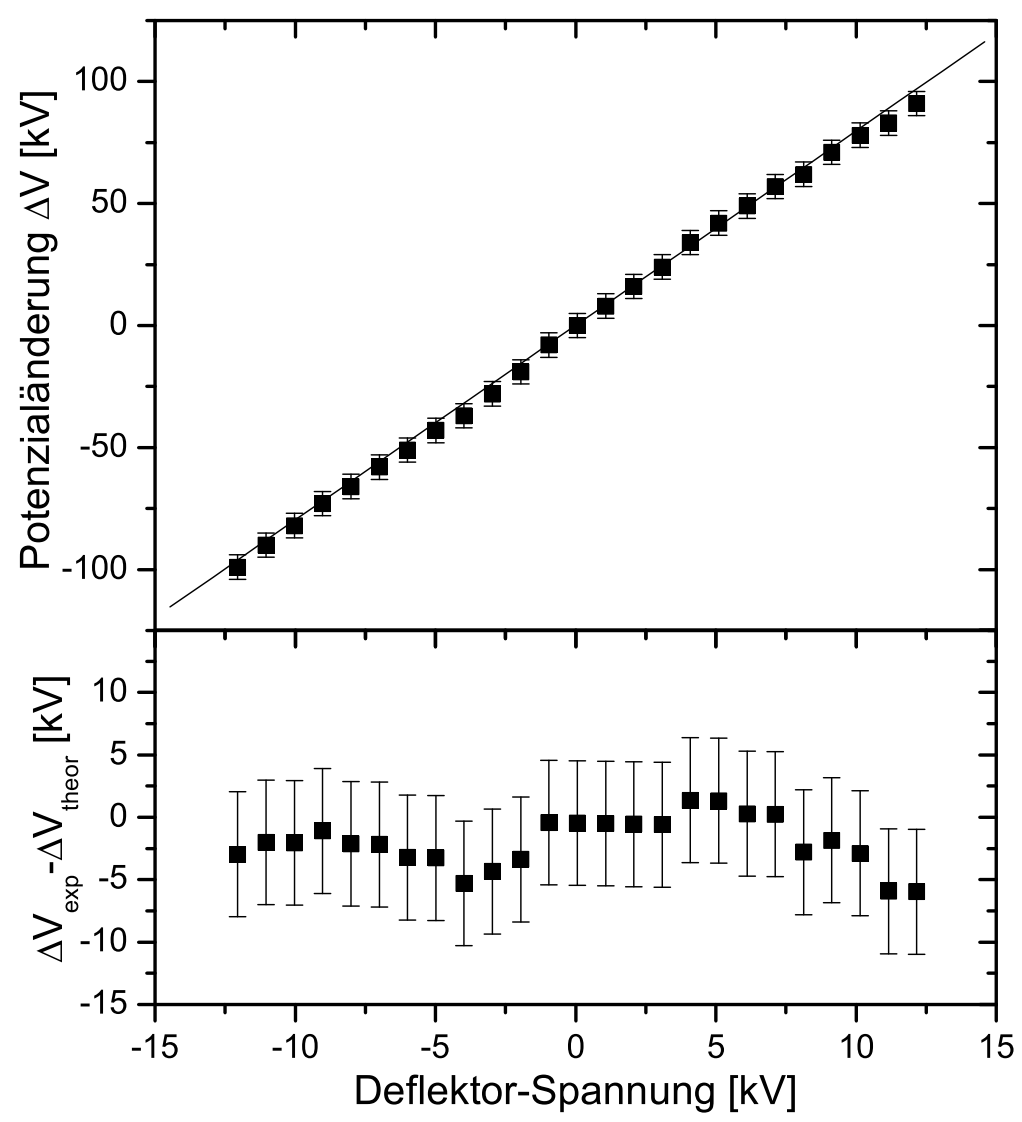

Abbildung 22: Experimenteller Verstärkungsfaktor $G_{0}($ exp. $)=7.97(8)$ für ${ }^{15} \mathrm{~N}$-Ionen , bestimmt mit dem Rotationsvoltmeter (GVM) 


\section{Nichtlineare Korrekturen}

Wie in Anhang B dargestellt wird, ist die lineare Beziehung zwischen der Änderung der Terminalspannung und der angelegten Deflektorspannung, die sich in einem konstanten Verstärkungsfaktor $\mathrm{G}_{0}$ für eine gegebene Ionensorte ausdrückt, nur für hohe Energien und schwere Ionen gut erfüllt. Tatsächlich hängt der Verstärkungsfaktor vom Ablenkwinkel $\alpha$ ab, der wiederum eine Abhängigkeit von der Aufenthaltsdauer im elektrischen Feld der Deflektoren aufweist und damit eine Funktion der Ionenenergie ist. Im Anhang B.6 wird für die Korrektur der Nichtlinearität von G für einen Protonenstrahl die Formel

$$
G(E)=G_{0}+G_{1}\left(V_{0}\right) \cdot U=G_{0}+\frac{41.7214}{V_{0}} \cdot U
$$

hergeleitet.

Für die in Abb. 20 dargestellte Messung bei einer Basis-Energie $\mathrm{E}_{b a s e}=750 \mathrm{keV}\left(\mathrm{V}_{0}=350 \mathrm{kV}\right)$ ist der Korrekturfaktor $\mathrm{G}_{1}=0.1113$. Damit liegen bei der maximal genutzten DeflektorSpannung von $\mathrm{U}=2.5 \mathrm{kV}$ die maximalen Abweichungen der Energie bei $\Delta E \leq 1.4 \mathrm{keV}$. Folglich können bei den hier zur Eichung benutzten kleinen Deflektor-Spannungen die nichtlinearen Korrekturen vernachlässigt werden.

\subsubsection{Messdatenerfassung mit KNRA}

Zur automatisierten Messdatenaufnahme einer NRA-Messung mit Hilfe der Amsel-Steerer wurde eigens das PC-Programm KNRA entwickelt. Bei KNRA handelt es sich um ein Realtime-Linux Programm, welches unter Nutzung der Qt ${ }^{\complement}(1.4)$-Bibliothek programmiert wurde und ein grafisches Frontend für die unter Linux weit verbreitete Benutzerumgebung KDE (K Desktop Environment) bietet. KNRA erlaubt sowohl eine Anzeige aller anfallenden Messdaten als auch eine online Untergrund-Korrektur der NRA-Spektren. Es folgt eine Beschreibung der Funktionalität des Messprogramms. Die beiden grundlegenden Bestandteile (Echtzeit-Betriebssystem und GUI-Bibliothek) des Systems zur Messdatenerfassung werden in Anhang $\mathrm{D}$ skizziert.

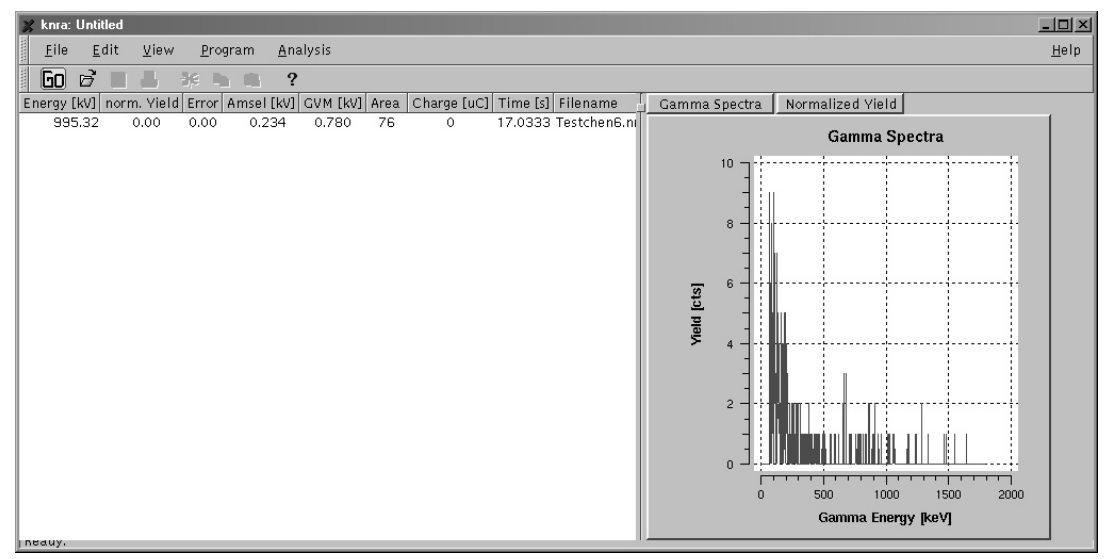

Abbildung 23: Das Hauptfenster des KNRA-Messprogramms 


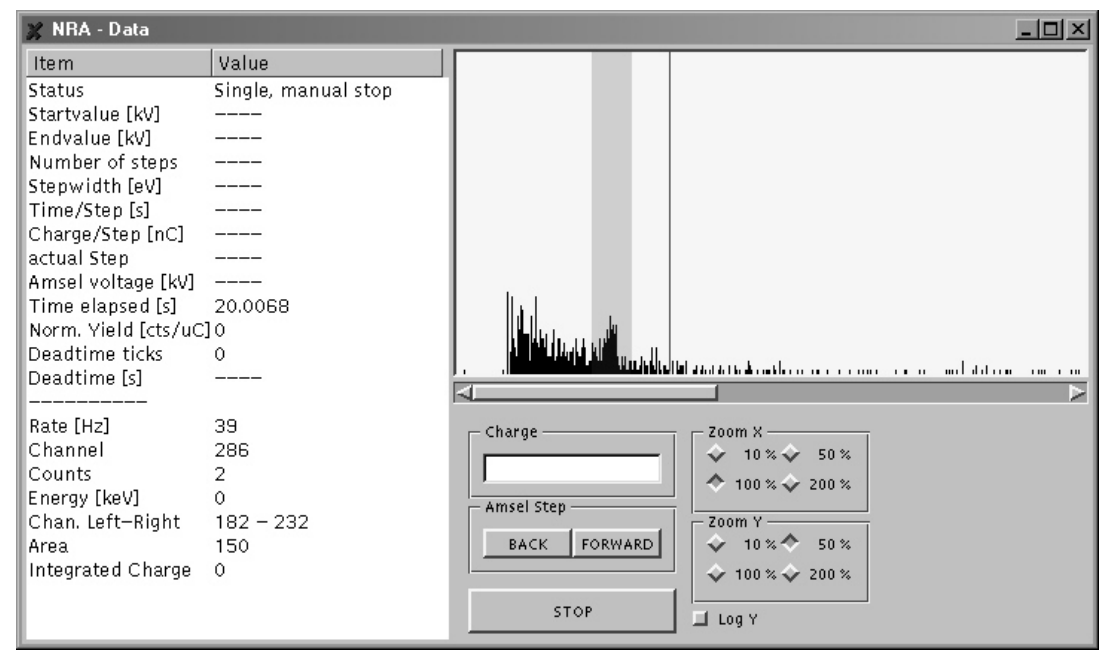

Abbildung 24: Messfenster des KNRA-Messprogramms

\section{Messprogramm KNRA}

Das NRA-Messprogramm (Abb. 23-25) bietet drei Modi zur automatisierten Messung: 1. Manuell, d.h. die Deflektor-Spannung wird von Hand am PC eingestellt und die Messung wird ebenfalls manuell gestoppt. Die Messdaten können dann einzeln abgespeichert werden. Dieser Modus bietet sich hauptsächlich für Tests und Messung des Strahlungsuntergrundes an.

2. Im sog. Autosave-Modus wird zwar die Deflektor-Spannung jeweils von Hand eingestellt, aber das Stoppen, Speichern und erneute Starten der Messung findet automatisch statt. Die Messung kann dabei sowohl zeit- als auch ladungsnormiert durchgeführt werden. Dieser Modus bietet sich an, wenn zunächst getestet werden soll, in welchem Bereich der Strahlenergie das Tiefenprofil vermessen werden soll.

3. Im Roboter-Modus schließlich wird eine vollständige NRA-Messung automatisch durchgeführt. Die Strahlenergie wird in einem vorher eingestellten Energiebereich in ebenfalls frei wählbarer Schrittweite beliebig oft zyklisch variiert. Auch hier kann die Messung zeitoder ladungsnormiert erfolgen.
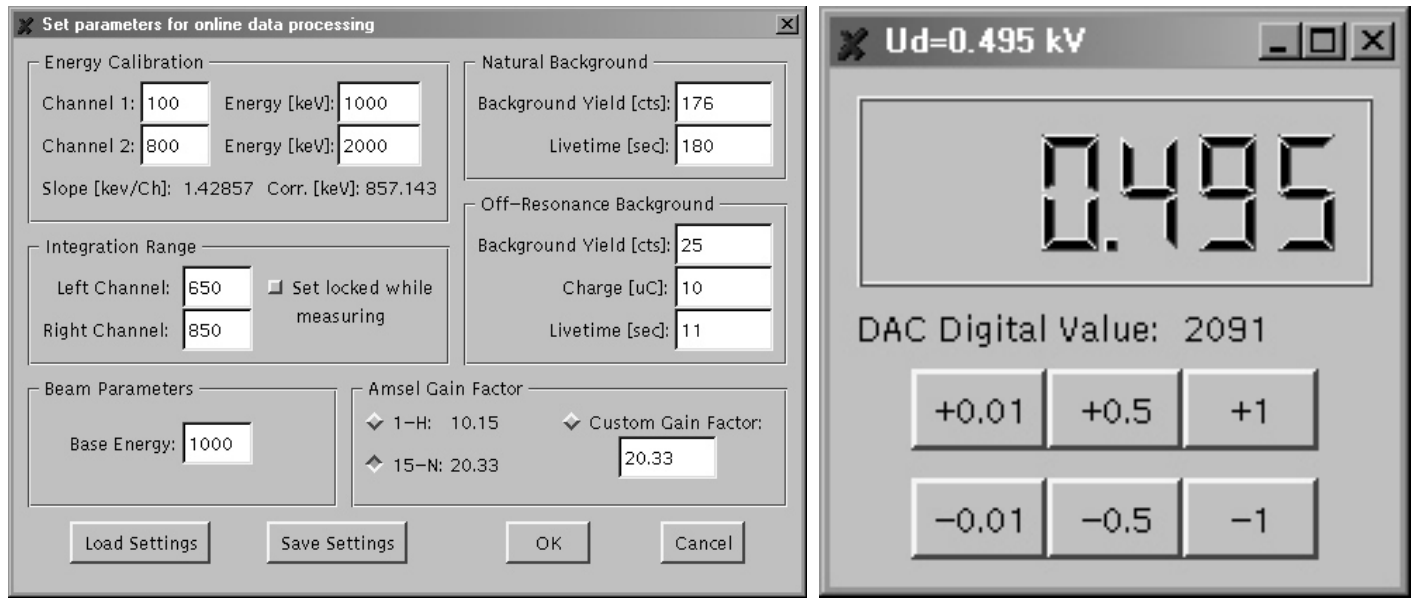

Abbildung 25: Analysefenster des KNRA-Messprogramms (links) und Direktansteuerung der Amselsteerer innerhalb des KNRA-Messprogramms (rechts) 


\subsubsection{Empfindlichkeit und Reproduzierbarkeit}

Ein wichtiges Maß für die Güte der NRA-Messungen ist die Nachweisempfindlichkeit der Methode. Die hohe Sensitivität beruht auf den schon im vorigen Abschnitt genannten starken Resonanzen des Wirkungsquerschnitts der verwendeten Kernreaktionen. Um die Nachweisempfindlichkeit der Messapparatur noch zusätzlich zu erhöhen, wurden die weiter oben dargestellten Maßnahmen zur Unterdrückung des natürlichen Strahlungsuntergrundes getroffen. Hier soll nun eine Abschätzung für die damit erreichbare Nachweisempfindlichkeit gegeben werden. Wie in Anhang C berechnet wird, gilt für die mit NRA minimal nachweisbare Konzentration $c_{\min }$ folgender Ausdruck:

$$
c_{\text {min }}=\frac{\dot{Y}_{R}}{K \cdot I}=\frac{1}{K \cdot I} \cdot \sqrt[3]{\frac{2 \dot{Y}_{U G}^{2}}{\left(\frac{\Delta Y_{R}}{Y_{R}}\right)^{2} t}}
$$

mit $\frac{\Delta Y_{R}}{Y_{R}}$ : relativer Fehler des Messsignals

$\dot{Y}_{R}$ : untergrundbereinigte Signalrate

$\mathrm{K}$ : Kammerkonstante

I : Messstrom

$\dot{Y}_{U G}:$ Untergrund-Signalrate

t : Messzeit

Als Beispiel soll mittels G1,17 die minimal nachweisbare Wasserstoff-Konzentration in Silizium berechnet werden. Wird für den maximal zulässigen relativen Fehler $\frac{\Delta Y_{R}}{Y_{R}}=0.5$ gewählt, so folgt für die folgenden typischen Werte:

$\dot{Y}_{U G}=0.98 \mathrm{cts} / \mathrm{s}, \mathrm{K}=21.126 \mathrm{cts} /(\mu \mathrm{C}$ at $\% \mathrm{H}$ in $\mathrm{Si}), \mathrm{I}=0.5 \mu \mathrm{A}$ und $\mathrm{t}=72 \mathrm{~s}$ für die Nachweisempfindlichkeit $c_{\text {min }}(\mathrm{H})=450$ atppm. Dieser Wert ist deutlich größer als der für die Heidelberger Messungen angegebene Wert von $c_{H}=80$ atppm [52]. Der Unterschied rührt dabei hauptsächlich von einer deutlich größeren mittleren Messzeit bei den Heidelberger NRA-Messungen her $\left(\mathrm{t}_{H D}=1000 \mathrm{~s}\right.$, vgl. Anhang C). Zur Überprüfung der Reproduzierbarkeit von NRA-Messungen sind in Abb. 26 vier NRA-Spektren der selben Probe dargestellt, die jeweils im Abstand von mehreren Wochen gemessen wurden.

In Tabelle 4 sind für zehn aufeinanderfolgende Messungen der gleichen $\mathrm{H}$-implantierten Siliziumprobe jeweils der Schwerpunkt der Gammaverteilung $\bar{x}$, die Halbwertsbreite $\Gamma$ und die aufintegrierte Gamma-Zählrate I angegeben. Im Idealfall einer fehlerfreien und zerstörungsfreien Messung sollten diese Werte konstant sein. Während die Werte für den Schwerpunkt der Gammaverteilung nur Abweichungen von $\Delta \bar{x} \leq 1 \%$ aufweisen, beträgt der mittlere prozentuale Fehler der Halbwertsbreite $\Delta \Gamma=7 \%$ und der mittlere prozentuale Fehler des Integrals $\Delta \mathrm{I}=4.5 \%$.

Ein Grund für diese relativ großen Abweichungen war eine Positionsänderung des Analysestrahls auf der Probenoberfläche während NRA-Messungen. Die in Abs. 2.2.2 beschriebene Zweifach-Viersektorenblende erlaubt es nun, die Position des Analysestrahls auf der Probe während der Messung zu kontrollieren. Nach dem Einbau der Blende wurde die Reproduzierbarkeit etwas verbessert. Der Einfluss des ${ }^{15} \mathrm{~N}$-Analysestrahls auf die Kristallstruktur der zu untersuchenden Proben wird in Abschnitt 6.3.2 diskutiert. 


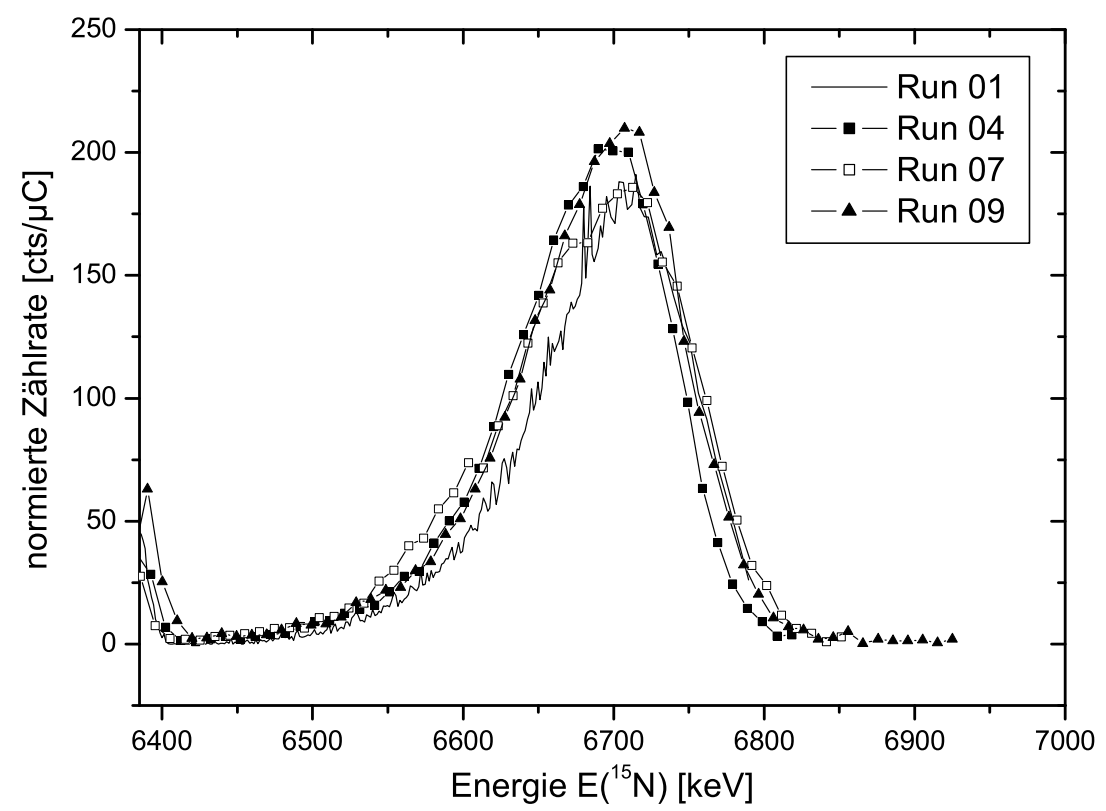

Abbildung 26: NRA-Rohspektren einer H-implantierten Si-Probe, die im Abstand von mehreren Wochen mehrmals vermessen wurde. Die integrierte Gamma-Zählrate zeigt trotz der hohen Bestrahlungsdosen von einigen $10^{17}{ }^{15} \mathrm{~N} / \mathrm{cm}^{2}$ pro Messung nur geringe Veränderungen.

\begin{tabular}{|c|l|l|l|l|l|l|}
\hline $\begin{array}{c}\text { Run } \\
\text { Nr. }\end{array}$ & \multicolumn{2}{|c|}{$\begin{array}{c}\text { Schwerpunkt } \\
x[\mathrm{keV}]\end{array}$} & \multicolumn{2}{c|}{$\begin{array}{c}\text { Breite } \\
\Gamma(\mathrm{FWHM})\end{array}$} & \multicolumn{2}{c|}{$\begin{array}{c}\text { Integral } \\
\text { I cts } /(\mathrm{keV} \cdot \mu \mathrm{C})]\end{array}$} \\
\hline \hline 1 & 6685 & $0 \%$ & 118 & $-4.8 \%$ & 23510 & $-14 \%$ \\
\hline 2 & 6666 & $-0.3 \%$ & 134 & $+8.1 \%$ & 26328 & $-3.8 \%$ \\
\hline 3 & 6676 & $-0.1 \%$ & 119 & $-4.0 \%$ & 26705 & $-2.4 \%$ \\
\hline 4 & 6696 & $+0.2 \%$ & 119 & $-4.0 \%$ & 27909 & $+2 \%$ \\
\hline 5 & 6689 & $+0.1 \%$ & 113 & $-8.9 \%$ & 27499 & $+0.5 \%$ \\
\hline 6 & 6672 & $-0.2 \%$ & 139 & $+12.0 \%$ & 28411 & $+3.8 \%$ \\
\hline 7 & 6680 & $-0.1 \%$ & 121 & $-2.4 \%$ & 29411 & $+7.5 \%$ \\
\hline 8 & 6696 & $+0.2 \%$ & 113 & $-8.9 \%$ & 28234 & $+3.2 \%$ \\
\hline 9 & 6700 & $+0.2 \%$ & 120 & $-3.2 \%$ & 28802 & $+5.3 \%$ \\
\hline 10 & 6691 & $+0.1 \%$ & 144 & $+16.1 \%$ & 26812 & $-2 \%$ \\
\hline \hline & 6685 & & 124 & & 27362 & \\
\hline
\end{tabular}

Tabelle 4: Werte für Schwerpunkt $\bar{x}$, Halbwertsbreite $\Gamma$ und Integral I der NRA-Rohspektren für zehn Messungen der gleichen H-implantierten Siliziumprobe, aufgenommen im Abstand von mehreren Wochen. Die Prozentangaben geben die prozentuale Abweichung von den in der letzten Zeile angegebenen Mittelwerten an. 


\section{Analysemethoden}

Nachdem im vorigen Abschnitt sehr detailliert auf den neu errichteten Low-Level NRAMessplatz im MaRPel-Labor eingegangen wurde, sollen im Folgenden die übrigen verwendeten Messmethoden kurz beschrieben werden.

\subsection{Rutherford-Rückstreuspektrometrie (RBS)}

Die Rutherford-Rückstreuspektrometrie (Rutherford Backscattering Spectroscopy, RBS) ist eine zerstörungsfreie Methode der Ionenstrahlanalytik, mit deren Hilfe vor allem kleinste Mengen schwerer Elemente in einer leichteren Matrix nachgewiesen werden können [53. 54. Die Methode beruht darauf, dass die zu untersuchende Probe mit leichten Projektilen $\left({ }^{4} \mathrm{He}^{2+}\right)$ mit Energien im $\mathrm{MeV}$-Bereich bestrahlt wird und die rückgestreuten Projektilionen mit einem Oberflächensperrschichtdetektor in einem Winkel möglichst nahe bei $180^{\circ}$ in Rückwärtsrichtung energieaufgelöst detektiert werden.

In Abb. 27 ist das Prinzip der RBS-Methode dargestellt. Ein Projektil mit der Anfangs-

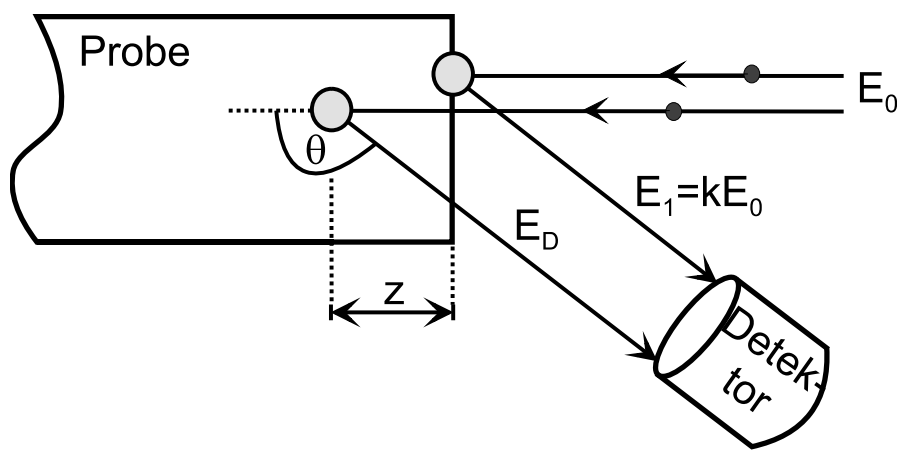

Abbildung 27: Prinzipskizze zum RBS-Messaufbau

energie $E_{0}$, das von der Targetoberfläche rückgestreut wird, hat nach dem Stoß die Energie $E=k \cdot E_{0}$, wobei für den kinematischen Faktor k aus Energie- und Impulserhaltung folgt:

$$
k=\left(\frac{\left(M_{1}^{2}-M_{2}^{2} \sin ^{2} \theta\right)^{1 / 2}+M_{1} \cos \theta}{M_{1}+M_{2}}\right)^{2}
$$

Dabei bezeichnet $M_{1}$ die Masse des Projektils, $M_{2}$ die Masse des Targetkerns und $\theta$ den Rückstreuwinkel. Aus Gl 18 folgt, dass bei bekannter Projektilenergie $E_{0}$, Projektilmasse $M_{1}$ und festgelegter Detektorgeometrie (Winkel $\theta$ ) die Masse des Targetatoms $M_{2}$ berechnet werden kann und somit die Zusammensetzung der Probe.

Findet der Stoßprozess erst in einer größeren Tiefe z statt, so verliert das Ion beim Durchgang durch die Materie aufgrund von Stößen mit den Targetatomen Energie und es wird im Detektor mit der niedrigeren Energie $E_{D}$ detektiert. In diesem Fall gilt:

$$
E_{D}=k\left(E_{0}-\int_{0}^{z} S\left(E_{0}\right) d z\right)-\int_{0}^{z / \cos \theta} S\left(k \cdot E_{0}\right) d z
$$


wobei $S(E)=-\frac{d E}{d z}$ den Energieverlust pro Weglängeneinheit, das sog. Bremsvermögen bezeichnet. Wie in Abschnitt 2.2.1 bereits dargestellt, muss zur Berechnung des Bremsvermögens bei Vorliegen unterschiedlicher Targetatome die Bragg-Regel (G1.2) angewendet werden. Die Energiedifferenz zwischen einem Ion, das an der Targetoberfläche rückgestreut wurde, und einem Ion, dessen Stoßprozess in der Tiefe z stattfand, beträgt $\Delta E=k E_{0}-E_{D}$. Der Wert von $\Delta E$ hängt von der Tiefe $\mathrm{z}$ ab und ist korreliert mit der Anzahl der Stoßpartner entlang der Ionenbahn, also mit der Atomdichte des Targetmaterials. Die „natürliche” RBS-Tiefenskala ist daher die Flächenbelegung in der Einheit $10^{15} \frac{\mathrm{at}}{\mathrm{cm}^{2}}$.

Abbildung 28 zeigt als Beispiel ein RBS-Spektrum einer Si(100)-Probe, die mit Xe-Ionen

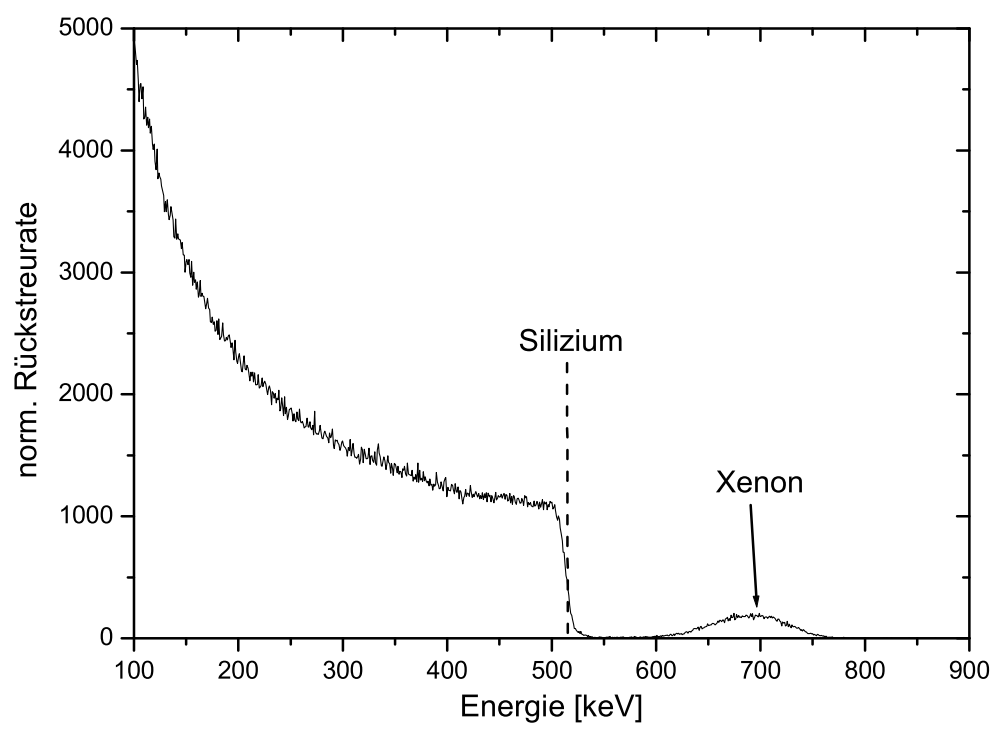

Abbildung 28: RBS-Spektrum einer Si(100)-Probe, die mit $450 \mathrm{keV}$ Xe-Ionen mit der Fluenz $\phi=5 \times 10^{16} \mathrm{Xe} / \mathrm{cm}^{2}$ bestrahlt wurde

der Fluenz $\phi=5 \times 10^{16} \mathrm{Xe} / \mathrm{cm}^{2}$ implantiert wurde. Deutlich ist das Signal der Streuung der $900 \mathrm{keV}{ }^{4} \mathrm{He}^{2+}$-Teilchen an der Siliziumoberfläche zu sehen. Vorgelagert, im Energiebereich $\mathrm{E}=620$ bis $760 \mathrm{keV}$ erscheint das gaußförmige Signal der implantierten Xenonionen. Aufgrund der größeren Masse der Xenonatome liegt der kinetische Faktor über dem der Si-Atome und das Xe-Signal erscheint daher bei einer höheren Rückstreuenergie.

\subsubsection{RBS-Tiefenprofile}

RBS wird oft benutzt, um Konzentrationsprofile implantierter schwerer Ionen zu bestimmen. Während der Nachweis der (leichten) Wasserstoffatome in der vorliegenden Arbeit mittels NRA durchgeführt wurde (vgl. Kap.2.2), wurden die Tiefenprofile der implantierten Xe-Ionen mittels RBS bestimmt.

Die Rückstreurate $Y\left(z\left(E_{D}\right)\right)$ von Projektilen mit einer Energie $E_{D}$, die in einer bestimmten Tiefe z rückgestreut wurden, beträgt

$$
Y\left(z\left(E_{D}\right)\right)=Y_{0} \frac{d \sigma}{d \Omega} \cdot E_{D}(z) \cdot \Omega \cdot(N \cdot z) .
$$


mit: $\mathrm{Y}_{0}$ : Proportionalitätskonstante, $\frac{d \sigma}{d \Omega}$ : Rutherford-Wirkungsquerschnitt, $\Omega$ : Detektorraumwinkel und $(\mathrm{N} \cdot \mathrm{z})$ Flächendichte.

Der Rutherford-Wirkungsquerschnitt für die Energie $E_{D}(z)$ ist gegeben durch den Ausdruck [55]:

$$
\frac{d \sigma}{d \Omega}=\left(\frac{1}{4 \pi \epsilon_{0}}\right)^{2} \cdot\left(\frac{Z_{1} Z_{2} e^{2}}{4 E_{D}(z)}\right)^{2} \frac{4}{\sin ^{4} \theta} \frac{\left(\sqrt{1-\left(\left(M_{1} / M_{2}\right) \sin \theta\right)^{2}}+\cos \theta\right)^{2}}{\sqrt{1-\left(\left(M_{1} / M_{2}\right) \sin \theta\right)^{2}}}
$$

$Z_{1}$ bezeichnet dabei die Kernladungszahl des Projektils und $Z_{2}$ die des Targets. Wegen der Proportionalität des Wirkungsquerschnitts (Gl 21) zu Z $Z_{2}^{2}$ ist die Rückstreurate für schwere Elemente sehr viel höher als für leichte Elemente. Außerdem nimmt die Rückstreurate bei niedrigeren Energien aufgrund der $\frac{1}{E^{2}}$-Abhängigkeit in Gl.21 stark zu.

Aus der Rückstreurate ergibt sich die Konzentration $C_{2}(z)$ des Elements $\left(\mathrm{Z}_{2}, \mathrm{~m}_{2}\right)$ in der Tiefe $\mathrm{z}$ wie folgt:

$$
C_{2}(z)=H\left(Q, \Omega, \Delta_{C h}\right) Y_{A} \frac{S\left(E_{D}(z)\right)}{\frac{d \sigma}{d \Omega} E_{D}(z)}
$$

Die Proportionalitätskonstante $\mathrm{H}$ ist eine Funktion der aufgebrachten Ladung $Q$ (Anzahl der Ionen), des Detektorraumwinkels $\Omega$, der Kanalbreite $\Delta_{C h}$, der stopping power in der Tiefe z $S\left(E_{D}(z)\right)$ und des Rutherford-Wirkungsquerschnitts $\frac{d \sigma}{d \Omega}$. Ist die Zusammensetzung einer Referenzprobe bekannt, so kann die Konzentration der Elemente in einem unbekannten Target bestimmt werden. In der vorliegenden Arbeit wurden alle RBS-Tiefenprofile mit Hilfe des Programms RUMP [56] entfaltet.

\subsubsection{RBS-Channeling}

Zur Untersuchung der Kristallinität der Si-Proben wurde die Rutherford-Rückstreu-Spektrometrie (Rutherford Backscattering Spectrometry, RBS) in Channeling-Geometrie (RBSC) verwendet. Alle RBS-C-Messungen wurden an der RBS-Kammer am Strahlrohr R30 des Göttinger Schwerionenimplantators IONAS durchgeführt. Der verwendete Messaufbau ist in [57] detailiert beschrieben.

Das Prinzip dieser Analysetechnik beruht darauf, dass die Rückstreuwahrscheinlichkeit von auftreffenden Projektilen bei einkristallinen Proben entlang der niedrigindizierten Kristallachsen deutlich reduziert ist gegenüber einem Einfall unter anderen Richtungen. Fällt die Einfallrichtung des Strahls mit einer Kristallachse zusammen, so werden die eintreffenden Projektile durch Kleinwinkel-Streuung am abstoßenden Potenzial der Atomreihen der kristallinen Matrix in größere Tiefen geführt. Dieser Channeling-Prozess von geladenen Teilchen im Kristallgitter wurde bereits 1912 von Stark vorhergesagt [58], jedoch erst 1963 von Robinson und Oen [59] experimentell nachgewiesen.

Liegt ein Kristall mit hoher Defektdichte oder amorphen Bereichen vor, so ist die Rückstreurate der Projektile gegenüber einem ungestörten Kristall deutlich erhöht. In Abb. 29 sind drei typische RBS-C-Spektren dargestellt. Zusätzlich ist die zugehörige Struktur der untersuchten Probe als Einsatz dargestellt.

Das „,virgin“-Spektrum in Abb. 29 entstand aus dem Einschuss von $900 \mathrm{keV} \mathrm{He}{ }^{2+}$-Teilchen entlang der (100)-Achse einer einkristallinen Si-Probe. Die Rückstreurate ist insgesamt 


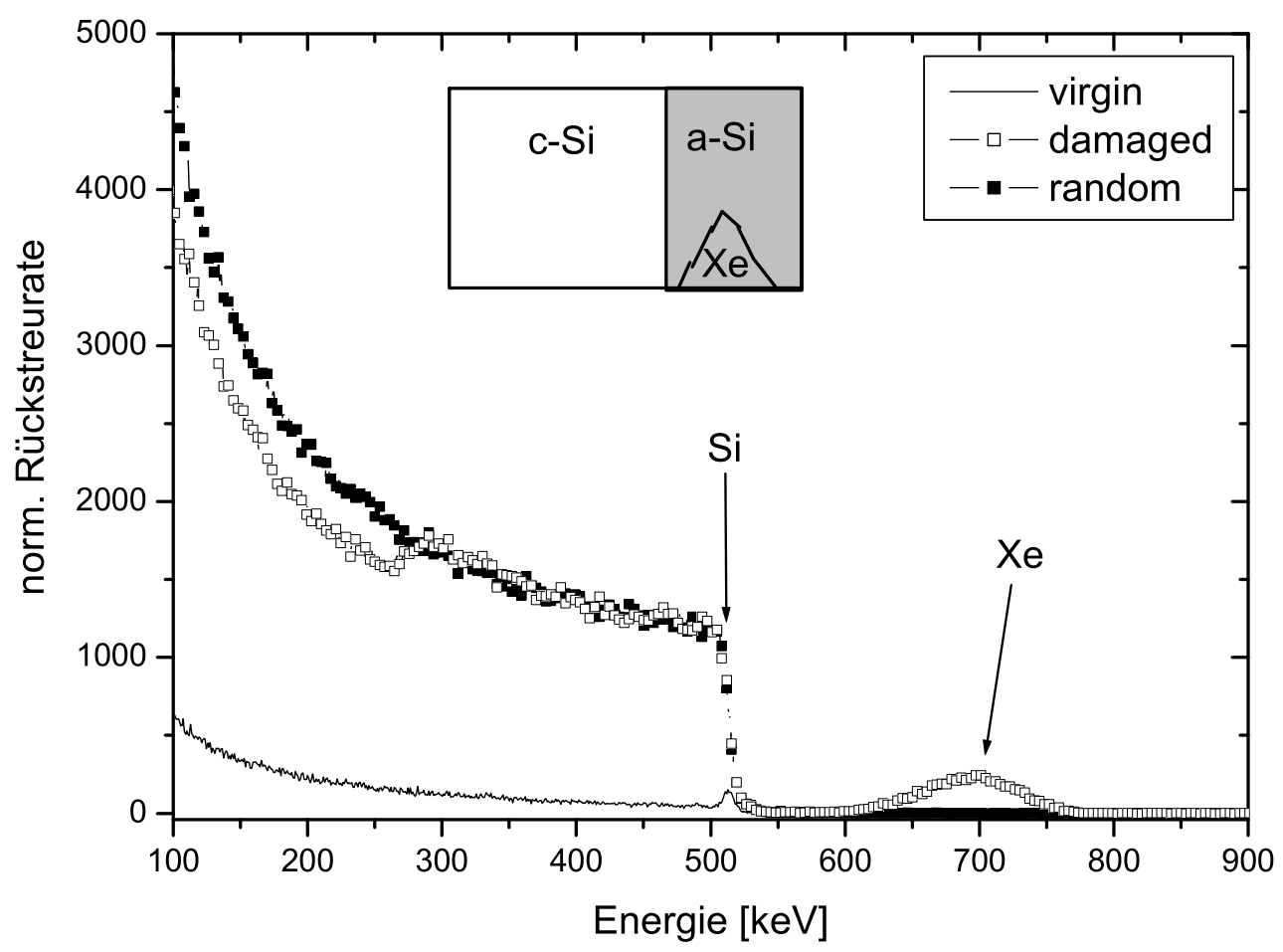

Abbildung 29: Beispiele für RBS-C-Spektren

sehr niedrig, nur an der Oberfläche zeigt sich ein Oberflächenpeak, der von den rückgestreuten Alphateilchen an den obersten Atomlagen der Probe herrührt. Das „random“Spektrum bildet den Einfall der Projektile unter rein zufälligen Richtungen ab. Dies wird durch eine ständige Rotation der zu untersuchenden Probe während der Messung erreicht. Das „damaged“-Spektrum schließlich zeigt den Fall einer Si-Probe mit einem amorphen

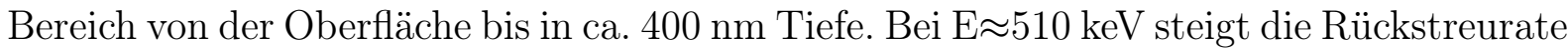
stark an und erreicht die Höhe des „,random”-Spektrums, d.h. bei der dargestellten Probe, die mit $\mathrm{Xe}^{2+}$-Ionen der Fluenz $\phi=5 \times 10^{15} \mathrm{Xe} / \mathrm{cm}^{2}$ bestrahlt wurde, hat sich eine amorphe Schicht gebildet. Zu niedrigeren Energien nimmt die Rückstreurate wieder ab, denn das tiefer liegende Si ist wieder einkristallin. Aus der Energie, bei der die Rückstreurate wieder abfällt, kann auf die Tiefe der Grenzschicht amorph/kristallin geschlossen werden. Die Rückstreurate sinkt hier nicht wieder auf den Wert des ,virgin”-Spektrums. Denn die Alphateilchen, die den amorphen Berich passiert haben, werden durch Stoßprozesse aus der ursprünglichen Bahn entlang der Kristallachse abgelenkt und somit ist die Bedingung für die Gitterführung nicht mehr erfüllt. Dieser von der Dicke der amorphen Schicht abhängige Effekt wird als „Dechanneling” bezeichnet. Aus dem Vergleich zwischen „damaged”und „,random"-Spektrum kann die normierte Schädigung $\chi$ in Abhängigkeit von der Tiefe z ermittelt werden. Die normierte Schädigung ist definiert durch:

$$
\chi_{n o r m}(z)=\frac{Y_{d a m}(z)-Y_{d e c}(z)}{Y_{\text {rand }}(z)-Y_{d e c}(z)}
$$

Dabei bezeichnet $\mathrm{Y}_{\text {dam }}(\mathrm{z})$ und $\mathrm{Y}_{\text {rand }}(\mathrm{z})$ die Rückstreuraten von „damaged“- und „random“Spektrum und $\mathrm{Y}_{\text {dec }}(\mathrm{z})$ bezeichnet den zunächst unbekannten Dechanneling-Anteil. Der Wert von $\mathrm{Y}_{d e c}(\mathrm{z})$ kann nach einem Algorithmus von Walker und Thomson [60] itera- 
tiv bestimmt werden. Dieser Algorithmus wird auch von dem Programm DAMAGE [61] benutzt, mit dem alle Schadensverteilungen der vorliegenden Arbeit bestimmt wurden. Erreicht die normierte Schädigung den Wert $\chi_{n o r m}=1$, so bedeutet dies, dass eine vollständig amorphisierte Schicht vorliegt. Aus dem Vergleich dieser Spektren ergibt sich die Möglichkeit, neben der Defektverteilung als Funktion der Tiefe auch die Dicke amorpher Schichten im Rahmen von Rekristallisationsexperimenten zu bestimmen.

In der vorliegenden Arbeit wird das Integral der normierten Schädigung von der Probenoberfläche bis in eine Tiefe $\mathrm{z}_{\max }$ durchweg mit $\mathrm{X}_{\text {tot }}$ bezeichnet:

$$
X_{\text {tot }}=\int_{0}^{z_{\max }} \chi_{n o r m}(z) d z
$$

\subsection{Oberflächenprofilometrie}

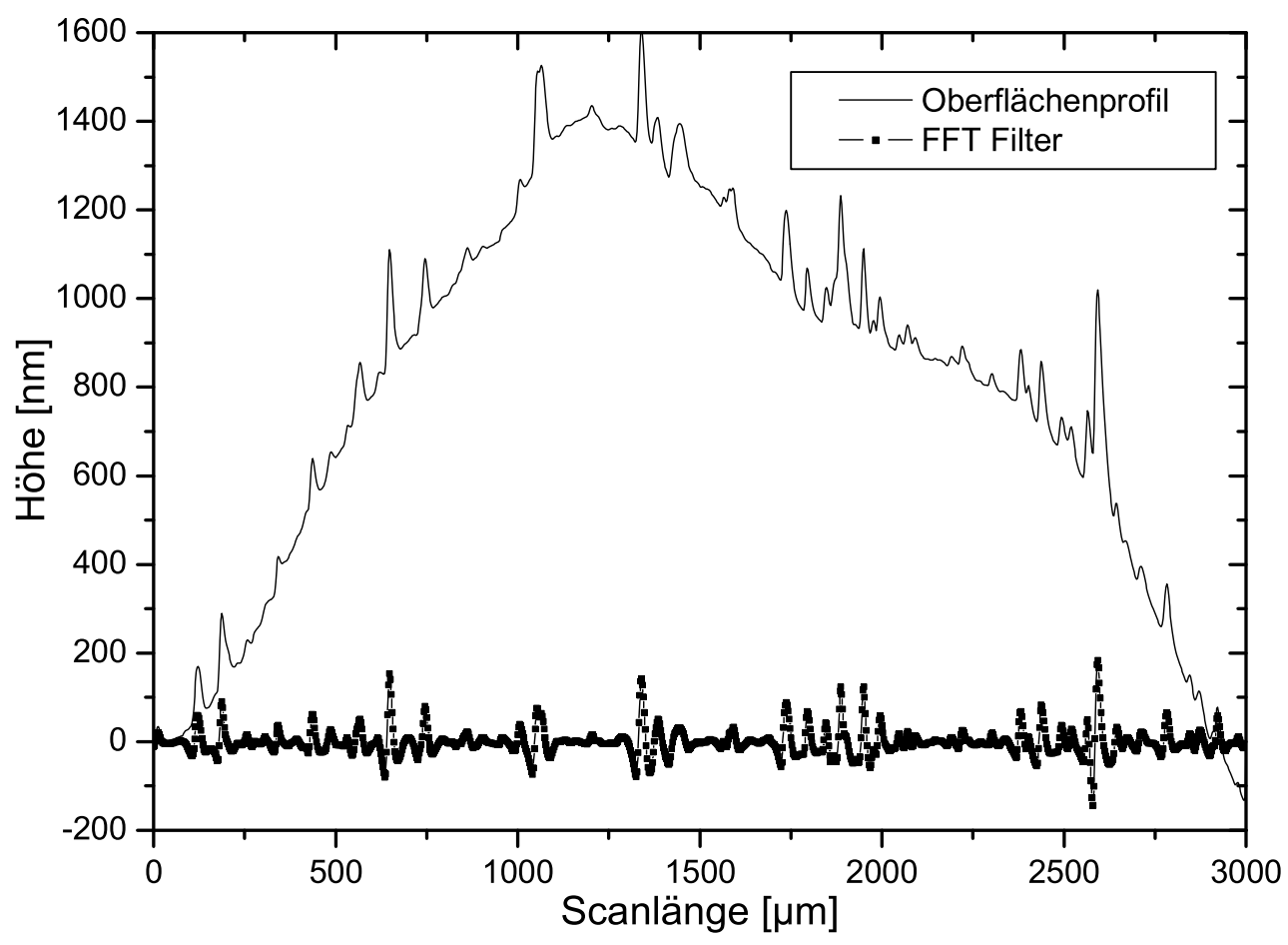

Abbildung 30: Oberflächenprofil (Linie) und Oberflächenrauigkeit (Symbole) einer Si(100)-Probe, die mit 36 Laserpulsen bei einer Laser-Fluenz von $F=3 \mathrm{~J} / \mathrm{cm}^{2}$ bestrahlt wurde. Die scheinbare Krümmung der Probe ist ein Artefakt der Skalierung: Die Abszisse umfasst drei Größenordnungen mehr, als die Ordinate. Die Welligkeit der Probenoberfläche wurde mit einem HochpassFourierfilter bereinigt.

Zur Bestimmung der Rauigkeit der Probenoberfläche nach der Ionenbestrahlung oder der Belichtung mit dem Laser wurde ein mechanisches Oberflächenprofilometer der Firma Veeco Instruments Inc. verwendet. Bei diesem Gerät des Typs DEKTAK ${ }^{3} \mathrm{ST}$ wird eine Diamantnadel mit einem Krümmungsradius von $1 \mu \mathrm{m}$ mit einem einstellbarem Andruck auf die Probenoberfläche abgesetzt und die Probe unter der Nadel fortbewegt. Die Höhenänderung der Nadel wird gemessen und über der zurückgelegten Wegstrecke 
aufgetragen. Abb. 30 zeigt ein Oberflächenprofil einer laserbestrahlten Si-Probe. Zur Bestimmung der Oberflächenrauigkeit muss der langwellige Anteil des dargestellten Profils (die Welligkeit der Probe) herausgefiltert werden. Mit Hilfe eines Fourier-Filters werden alle Strukturen mit einer Wellenlänge größer als $150 \mathrm{~nm}$ herausgefiltert. In Abb. 30 ist das Fourier-gefilterte Oberflächenprofil ebenfalls dargestellt. Nach dieser Korrektur kann gemäß

$$
R_{a}=\frac{1}{L} \int_{0}^{L}|H(x)-\bar{H}| d x
$$

der Rauigkeitswert $R_{a}$ bestimmt werden [62]. Dabei bezeichnet $H(x)$ die Höhe des korrigierten Oberflächenprofils, $\bar{H}$ die mittlere Höhe und L die Weglänge des Oberflächenscans. Für die in der vorliegenden Arbeit durchgeführten Rauigkeitsmessungen wurde stets eine Weglänge von $5000 \mu \mathrm{m}$ gewählt.

Zur Bestimmung der scheinbaren Rauigkeit, die durch Gebäudeschwingungen und das Aufösungsvermögen des Gerätes stets vorhanden ist, wurden sogenannte ,static scans" durchgeführt. Dabei wird die Nadel lediglich für eine bestimmte Zeit auf der Probenoberfläche abgesetzt, ohne das die Probe dabei bewegt wird. Bei sonst gleichen Einstellungen erhält man mit dieser Methode eine untere Nachweisgrenze für den $R_{a}$-Wert von $1 \mathrm{~nm}$. 


\section{Grundlagen zu H-Implantation und Laserhydrieren}

In diesem Kapitel werden die Material-Modifikationen beschrieben, die durch den Beschuss der Probenoberfläche mit Ionenstrahlen und durch Bestrahlung der Probe mit einem Excimer-Laser hervorgerufen werden.

Wie bereits erwähnt, war ein Ziel der vorliegenden Arbeit zu überprüfen, ob es bei der Laserbestrahlung von Materialien in Wasserstoffatmosphäre zu einem messbaren Wasserstoffeintrag in die Materialoberfläche kommt. Diese Experimente sind im Zusammenhang mit Untersuchungen zum Lasernitrieren zu sehen, die in den letzten Jahren durchgeführt wurden [63, 64, 65, 66, 3]. Während es beim Lasernitrieren von Metallen zu einer erwünschten Härtung der Oberfläche durch die Bildung einer Nitrid-Schicht kommt, ist ein hoher Wasserstoffgehalt in Metallen aus Sicht der Werkstoffherstellung eher unerwünscht. So führt die sogenannte Wasserstoff-Versprödung von Metallen zu einer geringeren Druckund Zugbelastbarkeit [67]. Dennoch wurden Versuche zum Laserhydrieren von Titanproben durchgeführt, da bekannt ist, dass Titan große Mengen Wasserstoff binden kann. Bei diesen Bestrahlungen handelt es sich nach unserer Kenntnis um die ersten Experimente zum „Laserhydrieren“ überhaupt. Die Experimente zum Laserhydrieren von Titan stellen daher einen ersten Test dar, welche Parameter (Laserfluenz, Pulszahl, Wasserstoffdruck) Einfluss auf den Wasserstoffeintrag haben.

Im Unterschied zu Metallen kann ein hoher Wasserstoffgehalt bei halbleitenden Materialien in vielen Fällen erwünscht sein. Besonders bei der Herstellung von elektronischen Bauelementen (z.B. TFT-Displays, „thin film transistors") auf Siliziumbasis wird Wasserstoff bewusst eingebracht, da sich dadurch die Defektdichte um mehrere Größenordnungen senken lässt [68]. Auch im Bereich der Solarzellenforschung wird der Einfluss des Wasserstoffgehalts auf die optische Stabilität der Schichten untersucht [69].

\subsection{Defekterzeugung und Amorphisierung durch Ionenstrahlen}

Bei der Bestrahlung eines Festkörpers mit energetischen Ionen tritt eine Fülle von Effekten auf. An dieser Stelle sollen nur die beiden Gesichtspunkte der Defekterzeugung und der Implantatverteilung diskutiert werden. Ein Teil der Siliziumproben wurde vor der Laserbestrahlung mit Xe- bzw. H-Ionen implantiert. Die Implantation der schweren Xe-Ionen hat dabei zum einen den Vorteil, dass schon eine relativ kleine Fluenz $\left(\phi_{\text {crit }}=10^{14} \mathrm{Xe} / \mathrm{cm}^{2}\right.$, bei $\mathrm{E}=450 \mathrm{keV}$ ) zur Erzeugung einer durchgehenden amorphen Deckschicht ausreicht. Zum anderen ist das Xe-Signal in RBS-Spektren deutlich getrennt vom Si-Signal (vgl. Abb. 28) und kann daher sehr gut als "Markerschicht" verwendet werden.

Die Implantation von H-Ionen geschah ebenfalls aus zwei Gründen: Einmal sollte auch hier die Si-Probe durch Ionenbestrahlung amorphisiert werden. Das definierte Einbringen von Wasserstoff vor dem Laserhydrieren erlaubte dann außerdem die Beobachtung der laserinduzierten Diffusion des bereits in der Si-Probe vorhandenen Wasserstoffs.

In den letzten Jahrzehnten hat sich a-Si:H zu einem wichtigen Rohstoff der Halbleiterindustrie entwickelt. Im Unterschied zu den üblichen Herstellungsverfahren von a-Si:H (z.B. „Plasma Enhanced Chemical Vapor Deposition“, PE-CVD) bietet die Ionenimplantation die Möglichkeit, den Wasserstoffgehalt sehr definiert einzustellen. Die Implantation 
von Wasserstoff in kristallines Silizium ermöglicht es, das Substrat zu amorphisieren und gleichzeitig Wasserstoff in die Probe einzubringen.

Ausgangspunkt aller Modelle zur ionenstrahlinduzierten Defekterzeugung ist die Tatsache, dass ein auf einen Festkörper treffendes Ion Targetatome von deren Gitterplätzen stoßen kann und dass diese wiederum selbst weitere Targetatome verlagern können. Auf diese Weise bildet sich eine Stoßkaskade im Targetmaterial aus. Eine SRIM2000-Simulation einer solchen Stoßkaskade ist in Abb. 31(b) für die Bestrahlung von Silizium mit XenonIonen der Energie $450 \mathrm{keV}$ gezeigt. Die Abbildung zeigt die Projektion der Stoßkaskade
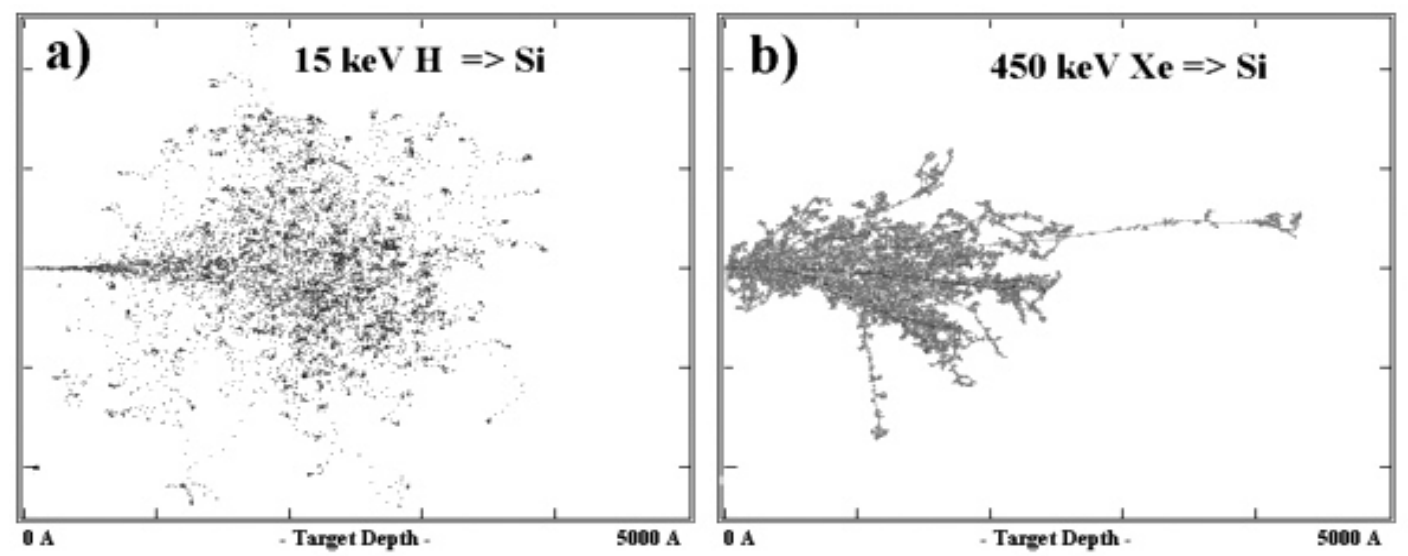

Abbildung 31: SRIM2000-Simulation [70] der Stoßkaskaden für die Implantation von $15 \mathrm{keV} \mathrm{H-}$ Ionen in Silizium (a) und $450 \mathrm{keV}$ Xe-Ionen in Silizium (b). Die Darstellung ist eine Projektion auf eine Ebene senkrecht zur Probenoberfläche.

auf eine Ebene senkrecht zur Probenoberfläche. Die Grundlage der SRIM2000-Simulation bildet die Annahme von binären elastischen Stoßfolgen. Werden diese Stoßfolgen raumfüllend, d.h. sind zu einem Zeitpunkt in einem Bereich des Targets alle Atome in Bewegung, so spricht man von Thermal-Spikes [71]. Diese Effekte treten besonders bei der Bestrahlung von schweren Targets mit massereichen Ionen auf und können hier, wie auch Abb. 31 (a) zeigt, vernachlässigt werden. Anhand der Abbildung wird ebenfalls deutlich, dass im Verlauf der Stoßkaskade die durch die Einschussrichtung der Ionen vorgegebene Vorzugsrichtung verloren geht. Molekulardynamik-Simulationen geben für die Dauer dieser Stoßkaskade einige Pikosekunden an [72, 73].

Die durch den Ionenstrahl in das Targetmaterial eingebrachte Energie kann insbesondere als Triebkraft für den Übergang in eine metastabile Phase der geschädigten Zone dienen. Bei kovalent gebundenen Materialien kann es zu einem ionenstrahlinduzierten Übergang in den amorphen Zustand kommen. Zur Beschreibung dieses Übergangs existiert eine ganze Reihe von Modellen.

Es wird unterschieden zwischen der ,direct-impact amorphization“, bei der die Amorphisierung innerhalb einer einzelnen Kaskade abläuft und den sogenannten „damage-overlap“ Modellen. Diese Modelle gründen auf der Überlegung, dass die eingeschossenen Ionen zunächst Punktdefekte im Bereich der Stoßkaskade erzeugen, deren Konzentration bei fortschreitender Bestrahlung zunimmt und letztlich zu Agglomeraten führt, wodurch die kristalline Struktur instabil wird und in den amorphen Zustand übergeht [74]. 
Dennis et al. hingegen beschreiben den Übergang von der kristallinen in die amorphe Phase aus rein energetischen Gesichtspunkten mit Hilfe der kritischen Energiedichte $\mathrm{H}_{\text {crit }}$ [75]. Mit $\mathrm{H}_{\text {crit }}$ wird diejenige Energiedichte bezeichnet, die durch den Ionenstrahl in das Targetmaterial deponiert werden muss, damit ein Bereich in den amorphen Zustand übergeht. Für die kritische Energiedichte gilt die folgende Beziehung:

$$
H_{\text {crit }}=F_{D}^{\max } \cdot \phi_{\text {crit }}
$$

Dabei bezeichnet $\phi_{\text {crit }}$ die so genannte ,kritische Fluenz", d.h. diejenige empirisch ermittelte Fluenz, oberhalb derer ein Bereich des Targetmaterials infolge der Ionenbestrahlung vollständig amorphisiert vorliegt. Die Größe $\mathrm{F}_{D}^{\max }$ gibt dabei die pro einfallendem Ion deponierte Energie in der Tiefe an, in der das Schadensprofil sein Maximum hat. Zur Bestimmung der deponierten Energiedichte in der Tiefe z führt Bolse [71] folgende Formel an:

$$
F_{D}(z)=E_{d} \cdot\left(2 n_{v a c}+n_{d i s}\right)
$$

Die pro Ion erzeugte Dichte an Leerstellen $\mathrm{n}_{v a c}$ und die pro Ion erzeugte Dichte an Ersetzungsstößen $\mathrm{n}_{\text {dis }}$ können mit Hilfe des Monte-Carlo-Programms SRIM2000 [70] bestimmt werden. Die Displacement-Schwelle $\mathrm{E}_{d}$ bezeichnet die Energie, die aufgewendet werden muss, um ein Atom von seinem Gitterplatz zu entfernen. Für die vorliegende Arbeit wurde für Silizium der Wert von $\mathrm{E}_{d}=15 \mathrm{eV}$ angenommen [70]. Die in Abb. 32 zusammengefassten

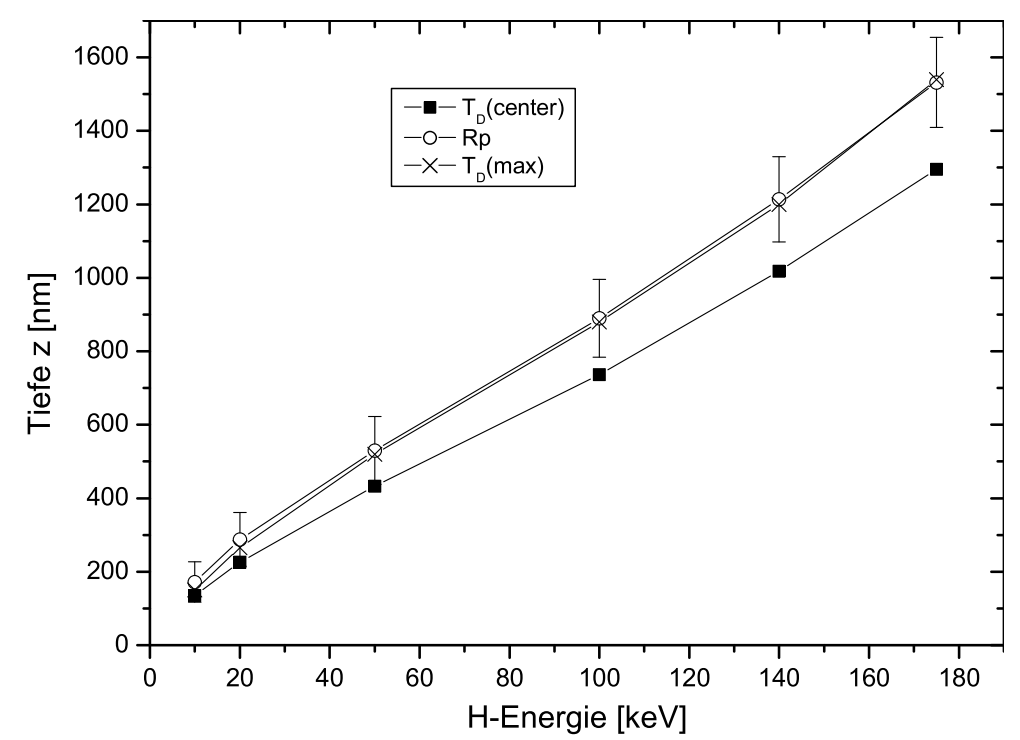

Abbildung 32: Ergebnisse von SRIM2000-Simulationen [70] zur H-Implantation von Si bei verschiedenen Energien. Dabei bezeichnet $R_{P}$ die (mittlere) Eindringtiefe der H-Ionen, $T_{D}$ (max) die Tiefe des Maximums der Schadensverteilung und $T_{D}$ (center) die Tiefe des Schwerpunkts der Schadensverteilung

SRIM2000-Simulationen für die Wasserstoffimplantation in Silizium im Energiebereich 10$180 \mathrm{keV}$ zeigen, dass das Maximum des Strahlenschadens näher an der Oberfläche liegen sollte, als die mittlere Reichweite der implantierten Ionen. Bis zu einer Implantationsenergie von $\mathrm{E}=50 \mathrm{keV}$ liegt der Schwerpunkt der Schädigung noch im Bereich der Breite des Implantationsprofils. Gemäß der Simulation kommt es bei der in dieser Arbeit benutzten Wasserstoff-Implantationsenergie von $\mathrm{E}=15 \mathrm{keV}$ zu einer Überschneidung von Schädigungsbereich und Wasserstoffprofil. 


\subsection{Grundlagen des Laserhydrierens}

In diesem Kapitel werden die physikalischen Grundlagen für den Prozess des Laserhydrierens dargelegt. Damit ist die Bestrahlung einer Festkörperoberfläche mit gepulster, intensiver Laserstrahlung in Wasserstoffatmosphäre gemeint. Der folgende Abschnitt beschreibt kurz die optischen Eigenschaften von Halbleitern, insbesondere im Hinblick auf die Absorption, die letztlich die Grundlage der Materialbearbeitung durch den Laser darstellt. Die daran anschließenden Abschnitte beschäftigen sich mit den Folgen der vom Material absorbierten Energie.

\section{Absorption von Laserstrahlung}

Elektromagnetische Strahlung mit Wellenlängen zwischen Infrarot und Ultraviolett zeigt einzig Wechselwirkung mit den Elektronen des bestrahlten Materials, da die Masse der Atomkerne zu groß ist, um auf die hohen Frequenzen der Laserstrahlung reagieren zu können. Für homogene Materialien kann eine Beschreibung der optischen Eigenschaften mittels makroskopischer Größen geschehen.

In absorbierenden Materialien muss der reelle Brechungsindex $\mathrm{n}$ durch den komplexen Brechungsindex N ersetzt werden

$$
N=n+i \kappa
$$

Durch die Parameter $\mathrm{n}$ und $\kappa$ ist die Reaktion des Materials auf die Laserstrahlung eindeutig festgelegt. Der Zusammenhang mit der Reflektivität $R$ und dem Absorptionskoeffizienten $\alpha$ ist gegeben durch

$$
R=\frac{(n-1)^{2}+\kappa^{2}}{(n+1)^{2}+\kappa^{2}} \quad \text { und } \quad \alpha=\frac{1}{l_{\alpha}}=\frac{4 \pi \kappa}{\lambda}
$$

Die Größe $l_{\alpha}$ wird als Absorptionslänge bezeichnet. Mit diesen Größen ergibt sich für die absorbierte Laserintensität in der Tiefe $\mathrm{z}$ und nach der Zeit $\mathrm{t}$, bei einer zeitlich veränderlichen Laserintensität (Laserpuls):

$$
I_{a b}(z, t)=\alpha I(t)(1-R) \exp (-\alpha z)
$$

Der Energieeintrag des Lasers pro Einheitsfläche wird als mittlere Laserfluenz F bezeichnet, für die in dieser Arbeit durchweg die Einheit $[\mathrm{F}]=\mathrm{J} / \mathrm{cm}^{2}$ benutzt wird. Beim Laserhydrieren der Proben wurden Fluenzen des Excimer-Lasers von $\mathrm{F}=0.5-6 \mathrm{~J} / \mathrm{cm}^{2}$ verwendet. Daraus ergibt sich mit der Pulsdauer $\tau_{L}=55$ ns unter der Annahme eines gaußförmigen Strahlprofils eine mittlere Laserintensität von $\bar{I}=\mathrm{F} / \tau_{L}=9-102 \mathrm{MW} / \mathrm{cm}^{2}$. Für eine tiefergehende Darstellung sei hier auf [76] verwiesen.

\section{Änderung der optischen Materialeigenschaften}

Nachdem im vorigen Abschnitt die beiden Größen $\mathrm{R}$ und $\alpha$ eingeführt wurden, soll nun kurz deren Abhängigkeit von der eingestrahlten Laserintensität beschrieben werden. All- 


\begin{tabular}{c|c|c} 
Element & $\mathrm{R}$ & $\alpha\left[\mathrm{cm}^{-1}\right]$ \\
\hline \hline $\mathrm{Ti}$ & 0.389 & $6.61 \times 10^{5}$ \\
\hline $\mathrm{a}-\mathrm{Si}$ & $\sim 0.69$ & $\sim 7.9 \times 10^{5}$ \\
\hline $\mathrm{c}-\mathrm{Si}$ & 0.6 & $1.48 \times 10^{6}$ \\
\hline
\end{tabular}

Tabelle 5: Reflektivität $R$ und Absorptionskoeffizient $\alpha$ für Titan [77], amorphes und kristallines Silizium [78] für die Wellenlänge $\lambda_{L}=308 \mathrm{~nm}$ des verwendeten Excimer-Lasers. Die Werte für $a$-Si wurden durch Interpolation der Angaben in [78] für $R$ und $\alpha$ bei $\lambda_{1}=250 \mathrm{~nm}$ und $\lambda_{2}=500 \mathrm{~nm}$ gewonnen.

gemein können Photonen zwei Arten von elektronischen Übergängen im Halbleitermaterial induzieren: Intraband-Übergänge zwischen gebundenen Elektronen und InterbandÜbergänge zwischen gebundenen und freien Elektronen. Zusätzlich besteht die Möglichkeit der resonanten Photon-Phonon-Kopplung. Liegt die Energie $E_{p h o t}=h \nu$ unterhalb der Bandlücke $E_{g}$ des Halbleiters, so sind nur Intraband-Übergänge und Phonon-Absorption als Wechselwirkungsprozesse möglich. Gilt für die Photonenergie jedoch $E_{\text {phot }} \approx E_{g}$, nimmt die Absorption zu, weil nun auch Interband-Übergänge möglich werden. Für diesen Fall muss zwischen Halbleitern mit direkter Bandlücke (z.B. GaAs) und Halbleitern mit indirekter Bandlücke (z.B. Si) unterschieden werden. Die unterschiedlichen BandBand-Übergänge führen zu verschiedenen Abhängigkeiten des Absorptionskoeffizienten $\alpha$ von der Energie der Photonen. Wichtig ist an dieser Stelle, dass auch in Materialien mit indirekter Bandlücke direkte Übergänge möglich sind, sofern die Photonen-Energie dafür ausreicht. Für kristallines Silizium beträgt die Energie der indirekten Bandlücke $E_{g}^{i n d}=1.11 \mathrm{eV}$ und die Energie des direkten Übergangs $E_{g}^{d i r}=3.4 \mathrm{eV}$. Die Photonenenergie des verwendeten Excimer-Lasers (s. Abs. 5.1) liegt mit $E_{p h o t}=4.02 \mathrm{eV}$ über diesen Werten, so dass auch direkte Übergänge möglich sind.

Für hohe Laserenergien ähnelt das optische Verhalten von Halbleitern demjenigen von Metallen. Die Effekte, die sich für die Energiedeposition bei Bestrahlung von Halbleitern mit hohen Laserintensitäten ergeben, lassen sich in zwei Kategorien einteilen. Zum einen spielt insbesondere bei Halbleitern die vermehrte Anregung freier Ladungsträger eine wichtige Rolle, zum anderen verändert auch das Aufheizen des Kristallgitters die optischen Eigenschaften.

Besonders bei Halbleitern mit indirekter Bandlücke (Si) hängt der Absorptionskoeffizient stark von der Anzahl der zur Verfügung stehenden Phononen ab. Dies ist der Grund für die im Vergleich zu kristallinem Silizium geringe Temperaturabhängigkeit des Absorptionskoeffizienten von amorphem Silizium. Die Bandlücke der meisten Halbleiter nimmt mit steigender Temperatur ab, wodurch ebenfalls die Absorption begünstigt wird. Zusätzlich zeigen viele Halbleiter (auch Silizium) als Schmelze ein metallisches Verhalten, wodurch Absorption und Reflektivität des Materials stark zunehmen [79].

\section{Laserinduzierter Temperaturanstieg}

Die vom Elektronensystem durch inverse Bremsstrahlung absorbierte Energie wird bei Halbleitern wie auch bei Metallen innerhalb von etwa 1 ps an das Phononensystem ab- 


\begin{tabular}{c|c|c} 
Element & $\mathrm{D}\left[10^{-2} \mathrm{~cm}^{2} / \mathrm{s}\right]$ & $\mathrm{l}_{T D}[\mu \mathrm{m}]$ \\
\hline \hline $\mathrm{Ti}$ & 0.075 & 64 \\
\hline $\mathrm{a}-\mathrm{Si}$ & 0.0097 & 23 \\
\hline $\mathrm{c}-\mathrm{Si}$ & 0.85 & 216 \\
\hline
\end{tabular}

Tabelle 6: Thermische Diffusivität D (bei Raumtemperatur) und thermische Diffusionslänge $l_{T D}$ für Titan, amorphes und kristallines Silizium [78]

gegeben. Infolgedessen kann der Laser als rein thermische Quelle aufgefasst werden [80]. Da die laterale Probenausdehnung im hier untersuchten Fall größer ist als die thermische Diffusionslänge $\mathrm{l}_{T D}=2 \sqrt{D \tau_{L}}$, deren Werte für die untersuchten Materialien in Tab.6 gegeben sind, kann die Zeitentwicklung des laserinduzierten Temperaturanstiegs $\mathrm{T}(\mathrm{t}, \mathrm{z})$ durch die eindimensionale Wärmeleitungsgleichung beschrieben werden:

$$
Q(z, t)=\rho(T) c_{P}(T) \cdot\left(\frac{\partial T(z, t)}{\partial t}-\frac{\partial}{\partial z}\left(D(T) \frac{\partial T(z, t)}{\partial z}\right)\right)
$$

Dabei bezeichnet Q(z,t) die vom Laser eingekoppelte Energie pro Volumen- und Zeiteinheit. Da sowohl die Dichte $\rho(T)$, als auch die spezifische Wärmekapazität $c_{P}(T)$ und die thermische Diffusivität $\mathrm{D}(\mathrm{T})$ temperaturabhängig sind, ist die eindimensionale Wärmeleitungsgleichung (Gl. 30) nichtlinear und kann nur numerisch gelöst werden. Dazu wurde das in [81, 3] beschriebene Verfahren der finiten Differenzen verwendet, mit dessen Hilfe sich die zeitliche Entwicklung der Oberflächentemperatur, sowie der zeitliche Verlauf der Aufschmelztiefe simulieren lassen.

Bei dem in der vorliegenden Arbeit benutzten PC-Programm [82, 63, 64, 65] wurde die am Schmelzpunkt einsetzende Verdampfung des Materials berücksichtigt, denn das Verdampfen führt neben dem Materialabtrag auch zu einem Energieverlust der bestrahlten Oberfläche. Die Hauptfehlerquelle der hier gezeigten Simulationen ergibt sich aus der Vernachlässigung der Plasmabildung und der damit verbundenen Effekte. Die Werte für die thermophysikalischen Eigenschaften des Titans wurden aus [83, 84, 85] entnommen, diejenigen des Siliziums aus [78].

Abbildung 33 zeigt eine Simulation der Laserbestrahlung von Titan bei der Laserfluenz $\mathrm{F}=4 \mathrm{~J} / \mathrm{cm}^{2}$, mit der alle in dieser Arbeit beschriebenen Titanproben laserhydriert wurden. Im oberen Teil ist die Oberflächentemperatur $\mathrm{T}_{\text {surf }}$ als Funktion der Zeit dargestellt. Gemäß dieser Simulation wird die Schmelztemperatur $\mathrm{T}_{\text {melt }}=1943 \mathrm{~K}$ bereits nach 10 ns überschritten, die Siedetemperatur $\mathrm{T}_{\text {boil }}=3560 \mathrm{~K}$ nach 20 ns. Der untere Teil von Abb. 33 zeigt eine Auftragung der simulierten Aufschmelztiefe $z_{m e l t}$ als Funktion der Zeit, ebenfalls für die Laserfluenz $\mathrm{F}=4 \mathrm{~J} / \mathrm{cm}^{2}$. Die maximale Aufschmelztiefe von $1.43 \mu \mathrm{m}$ wird demnach 250 ns nach Einsetzen des Laserstrahls erreicht. Aus der gezeigten Simulation kann eine maximale Aufheizrate von $\mathrm{r}_{\text {heat }}=\mathrm{dT} / \mathrm{dt}=10^{11} \mathrm{~K} / \mathrm{s}$ und eine maximale Abkühlrate von $\mathrm{r}_{\text {cool }}=-3 \times 10^{10} \mathrm{~K} / \mathrm{s}$ abgeleitet werden.

Die Ergebnisse der Simulationsrechnung für kristallines Silizium sind in Abb. 34 dargestellt. Die Auftragung zeigt die simulierte Oberflächentemperatur $\mathrm{T}_{\text {surf }}$ als Funktion der Zeit t für verschiedene Laserfluenzen F. Zusätzlich sind die Schmelztemperatur $\left(\mathrm{T}_{\text {melt }}=1685 \mathrm{~K}\right)$ und die Verdampfungstemperatur $\left(\mathrm{T}_{\text {vap }}=3265 \mathrm{~K}\right)$ des kristallinen Siliziums eingezeichnet. $\mathrm{Ab}$ einer Temperatur von $\mathrm{T}_{\text {cryst }}=823 \mathrm{~K}$ wird eine Rekristallisation 


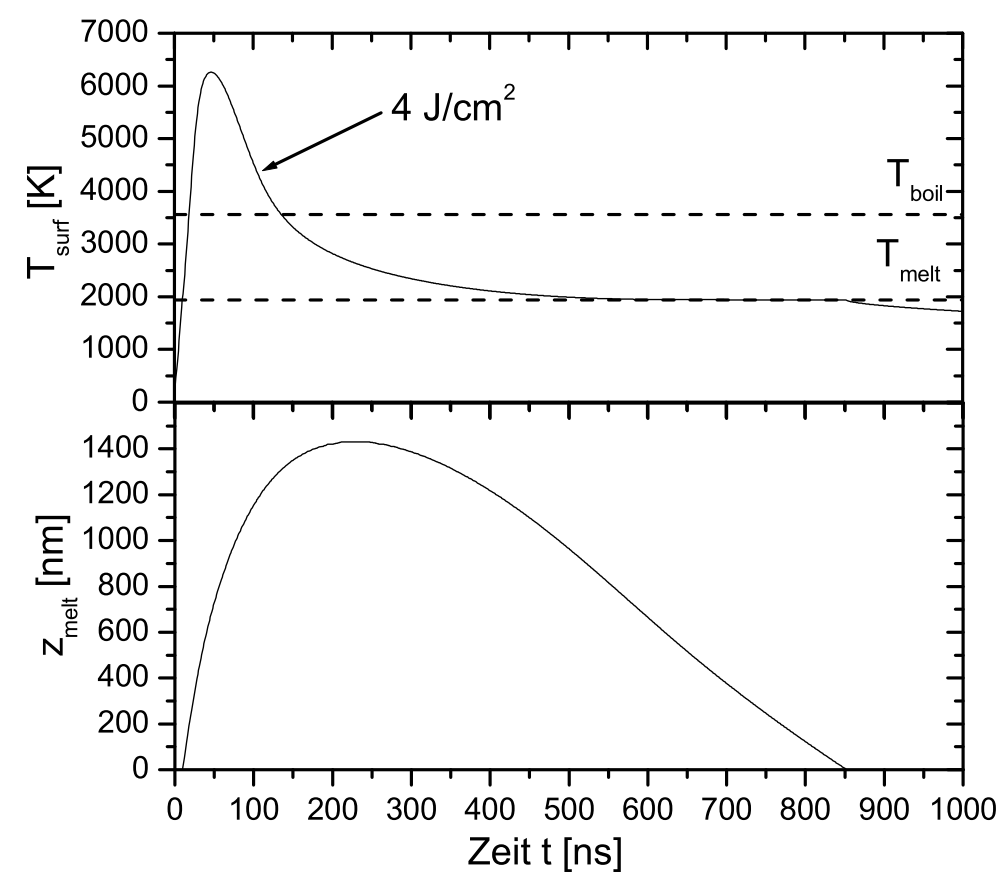

Abbildung 33: Simulation der Oberflächentemperatur $T_{\text {surf }}(t)$ (oben) und der Aufschmelztiefe $z_{\text {melt }}(t)$ (unten) für Titan

amorphisierter Siliziumschichten in makroskopischem Maßstab beobachtet [86]. Auf die laserinduzierte Rekristallisation wird in Abs. 4.3 noch genauer eingegangen.

Es zeigt sich, dass eine Laserfluenz von $0.5 \mathrm{~J} / \mathrm{cm}^{2}$ für ein Aufschmelzen der Probenoberfläche nicht ausreicht. Mit zunehmender Laserfluenz steigt auch der Maximalwert der Oberflächentemperatur $\mathrm{T}_{\text {sur }}^{\max }$ an. Oberhalb von $\mathrm{F}=3 \mathrm{~J} / \mathrm{cm}^{2}$ überschreitet die Temperatur an der Oberfläche den Siedepunkt von Silizium. Für die Bestrahlung mit $\mathrm{F}=5 \mathrm{~J} / \mathrm{cm}^{2}$ ergibt die Simulation eine maximale Aufheizrate von $\mathrm{r}_{\text {heat }}=10^{11} \mathrm{~K} / \mathrm{s}$ und eine maximale Abkühlrate von $\mathrm{r}_{\text {cool }}=-2 \times 10^{10} \mathrm{~K} / \mathrm{s}$.

Abbildung 35 zeigt die Ergebnisse von Simulationsrechnungen für die Laserbestrahlung von amorphem Silizium. Aufgrund der im Vergleich zu c-Si geringeren Schmelztemperatur des amorphen Siliziums $\left(\mathrm{T}_{\text {melt }}=1420 \mathrm{~K}\right)$ reicht schon eine Laserfluenz von $\mathrm{F}=0.5 \mathrm{~J} / \mathrm{cm}^{2}$ für ein Aufschmelzen der Probenoberfläche aus. Der Siedepunkt wird ebenfalls schon bei einer kleineren Laserfluenz von $\mathrm{F}=3 \mathrm{~J} / \mathrm{cm}^{2}$ erreicht. Ein weiterer Unterschied gegenüber der Laserbestrahlung von kristallinem Silizium besteht in der wesentlich geringeren Abkühlrate. Die maximale Abkühlrate des a-Si ist mit $\mathrm{r}_{\text {cool }}=-2 \times 10^{10} \mathrm{~K} / \mathrm{s}$ zwar nur ein wenig kleiner als im kristallinen Fall, jedoch wird bei der Diskussion der Ergebnisse in Kap. 7 gezeigt werden, dass die Geschwindigkeit der Erstarrungsfront $\mathrm{v}_{s / l}$ zwischen der schon festen und der noch geschmolzenen Phase bei amorphem Silizium sehr viel kleiner ist als bei kristallinem Silizium. 


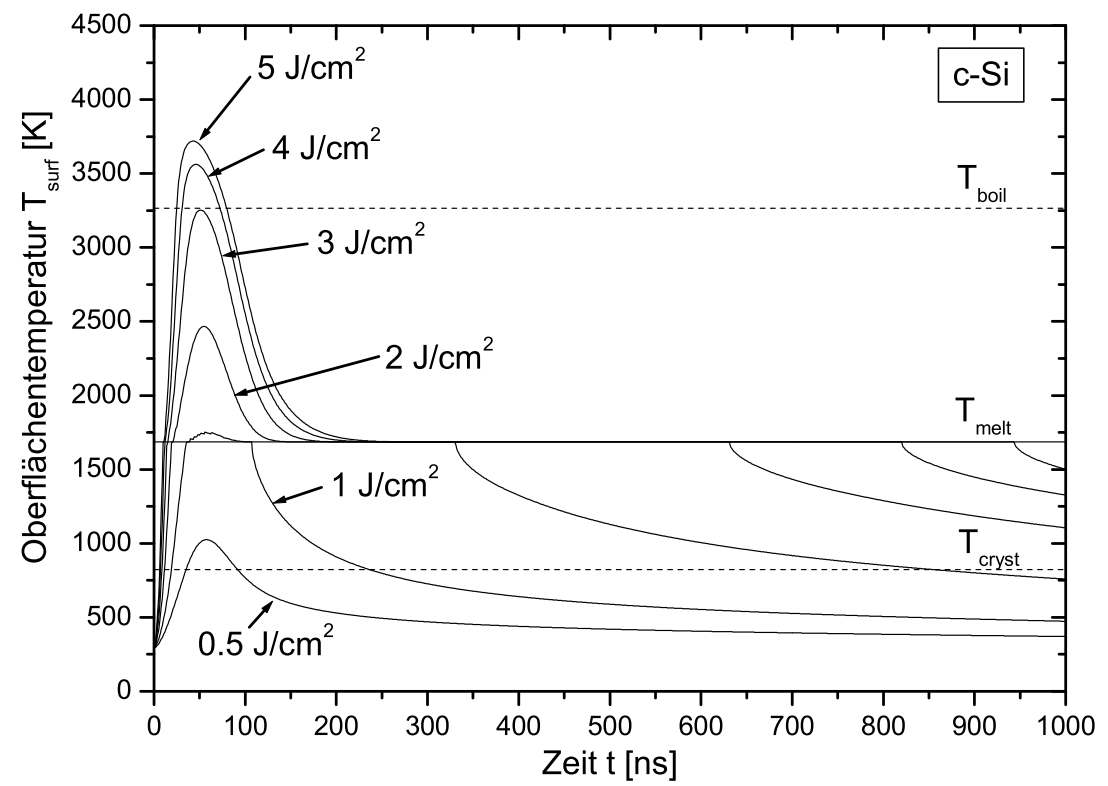

Abbildung 34: Simulation der Oberflächentemperatur $T_{\text {surf }}$ als Funktion der Zeit $t$ für die Laserbestrahlung von $\mathrm{c}$-Si im Fluenzbereich $0.5 \mathrm{~J} / \mathrm{cm}^{2} \leq F \leq 5 \mathrm{~J} / \mathrm{cm}^{2}$

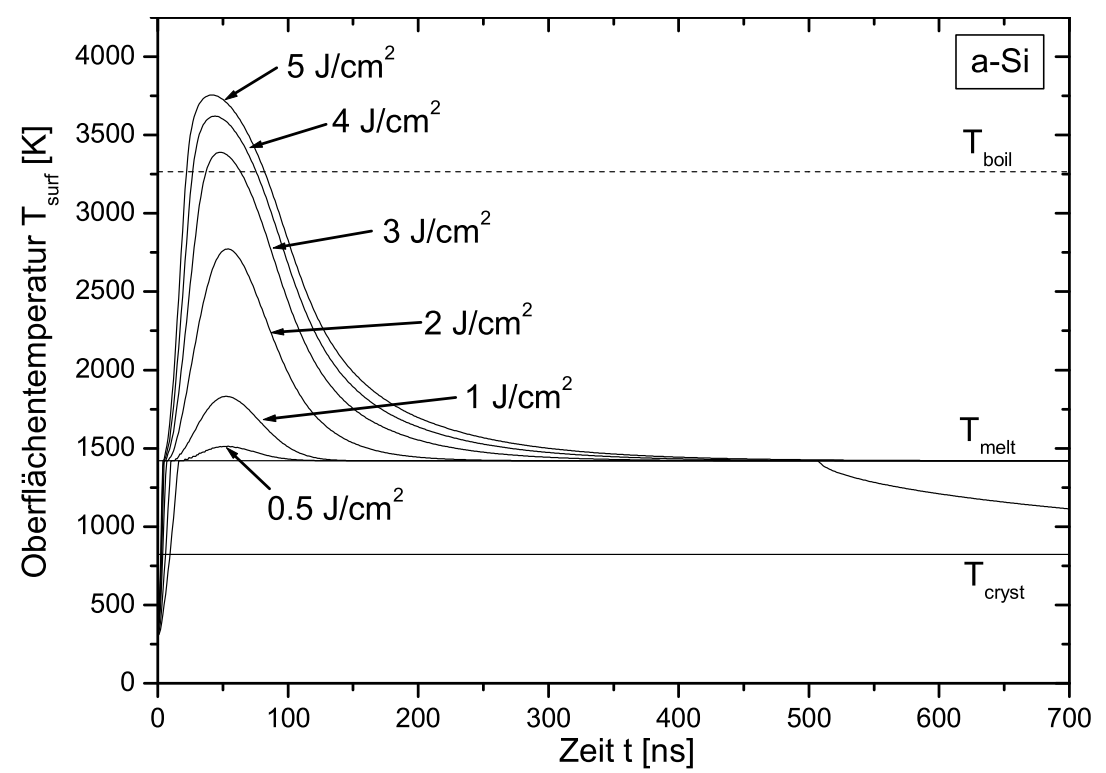

Abbildung 35: Simulation der Oberflächentemperatur $T_{\text {surf }}$ als Funktion der Zeit t für die Laserbestrahlung von a-Si im Fluenzbereich $0.5 \mathrm{~J} / \mathrm{cm}^{2} \leq F \leq 5 \mathrm{~J} / \mathrm{cm}^{2}$ 


\section{Plasmabildung}

Selbst bei den höchsten Laserintensitäten von $\mathrm{I}_{\max }=102 \mathrm{MW} / \mathrm{cm}^{2}$, die in dieser Arbeit verwendet wurden, kommt es nicht zum direkten Zünden eines Plasmas in der Wasserstoff-Atmosphäre.

Die Photonenenergie des verwendeten Lasers liegt mit $\mathrm{E}_{\text {phot }}=4.025 \mathrm{eV}$ unterhalb der Ionisationsenergie von molekularem Wasserstoff von $\mathrm{E}_{\text {ion }}\left(\mathrm{H}_{2}\right)=15.426 \mathrm{eV}$ [77]. Dennoch können durch Multiphoton-Absorption oder den Effekt des ,self-focussing“ zur Ionisierung der Gas-Atome an der Festkörperoberfläche kommen [87].

Der Plasmabildung geht dabei immer ein Verdampfen der bestrahlten Oberfläche voraus (,thermal runnaway") [88, 89]. Dieser Dampf spielt eine wichtige Rolle bei der Materialmodifikation mittels Laserstrahlung. Bei kleinen Laserintensitäten ist der Dampf im wesentlichen transparent für die Laserstrahlung. Ab ca. $10 \mathrm{MW} / \mathrm{cm}^{2}$ wird der Dampf ionisiert und der Absorptionskoeffizient erhöht sich. Ab einer Grenzintensität $\mathrm{I}_{P}$ wird die Absorption so groß, dass sich aus dem heißen Dampf ein Plasma bildet. Dieses heiße Plasma expandiert von der Oberfläche aus entgegengesetzt zur einfallenden Laserstrahlung und wirkt stark absorbierend (,laser supported absorption wave“, LSA). Anhand der Ausbreitungsgeschwindigkeit der Plasmafront unterscheidet Raizer [90] weiter zwischen der „laser supported combustion wave“ (LSC), die sich mit Unterschallgeschwindigkeit (bezogen auf die Gasatmosphäre) bewegt und der sogenannten „laser supported Detonation wave" (LSD), deren Ausbreitungsgeschwindigkeit die Schallgeschwindigkeit der umgebenden Gasatmosphäre überschreitet. Bei Untersuchungen zur Effizienz des Lasernitrierens hat sich der LSC-Bereich als besonders vorteilhaft herausgestellt [3, 65]. Aus diesem Grund wurden auch die hier gezeigten Untersuchungen zum Laserhydrieren größtenteils bei Laserfluenzen durchgeführt, die im LSC-Bereich liegen. Das stationäre Plasma direkt über der Materialoberfläche ermöglicht eine gute thermische Ankopplung an das bestrahlte Material.

\section{Materialtransport in der Schmelze}

Ein lateraler Materialtransport innerhalb der durch Laserstrahlung aufgeschmolzenen Oberfläche beruht hauptsächlich auf zwei Prozessen. Zum einen führt die Temperaturabhängigkeit der Oberflächenspannung $\sigma(T)$ durch Inhomogenitäten des Laser-Profils zum „Thermokapillaren Effekt". Andererseits kommt es durch den Überdruck im bestrahlten Bereich zu einem Kolbeneffekt (,Piston Effekt"), wodurch sich die geschmolzene Oberfläche absenkt, was zu einem Materialfluss aus dem Zentrum in die Randbereiche des Laserflecks führt. Der Piston-Mechanismus wurde von von Allmen untersucht [76], der folgende Formel für die radiale Geschwindigkeit der Schmelze ableitete:

$$
v=\sqrt{\frac{2 \Delta p}{\rho}}
$$

Dabei bezeichnet $\Delta p=p_{p}+p_{r}$ die Summe aus Plasmadruck und Rückstoßdruck. Eine Abschätzung für die hier verwendeten Laserintensitäten ergibt für die laterale Geschwindigkeit die Größenordnung $100 \mathrm{~m} / \mathrm{s}$. 


\section{Wasserstoffaufnahme}

Für das Einbringen von Wasserstoff in die Probe durch Laserhydrieren wird an dieser Stelle der gleiche Prozess angenommen, der auch beim Lasernitrieren zugrunde gelegt wird [3, 81].

Durch die Einstrahlung hoher Laserintensitäten wird das Material im oberflächennahen Bereich (ca. einige $\mu \mathrm{m}$ ) aufgeschmolzen. Durch die Evaporation von Elektronen und Materialdampf bildet sich in der Nähe der Materialoberfläche ein Plasma (s. Abs. „Plasmabildung"), welches für das Einbringen von Gasatomen ins Material zwei wichtige Funktionen erfüllt. Erstens stellt das Plasma eine oberflächennahe Wärmequelle dar und hält somit die geschmolzene Phase aufrecht. Zweitens werden erst durch das Plasma Gasmoleküle dissoziiert, welche dann entweder durch Konvektion oder durch Diffusion in der Schmelze tiefer in das Material eindringen.

\subsection{Laserinduzierte Rekristallisation von Silizium}

Mit (Re-) Kristallisation wird die Umwandlung eines amorphen Materials in die geordnete kristalline Phase bezeichnet. Dieser Umordnungsprozess kann nur unter bestimmten thermodynamischen Vorraussetzungen stattfinden. Der Grund dafür, dass es überhaupt zu einer Umwandlung vom amorphen in den kristallinen Zustand kommen kann, ist eine höhere Gibbssche Freie Enthalpie $G_{a m}$ der amorphen Phase als die der kristallinen Phase $\mathrm{G}_{c r}<\mathrm{G}_{a m}$. Obwohl die Differenz $G_{a m}-G_{c r} \approx 0.1 \mathrm{eV} /$ atom bei $300 \mathrm{~K}$ [91] für Silizium nur gering ist, stellt sie dennoch die Triebkraft zur Umordnung der Bindungswinkel und atomaren Abstände dar. Die Kinetik dieser Prozesse ist normalerweise sehr langsam, kann aber durch Temperaturerhöhung stark beschleunigt werden. Durch die Zunahme der atomaren Schwingungen bei einer Temperaturerhöhung nimmt die Viskosität des Materials ab und die Umordnung der Atome wird begünstigt.

Die Grenzfläche zwischen amorphem und kristallinem Material bewegt sich bei der Rekristallisation mit einer konstanten Geschwindigkeit $\mathrm{v}_{a / c}$. Die Geschwindigkeit ist proportional zur Sprungrate der Atome zwischen dem amorphen und kristallinen Bereich, und es gilt:

$$
v_{a / c}=v_{0} \cdot \exp \left(-\frac{E_{a}}{k_{B} T}\right)
$$

Dabei bezeichnet $\mathrm{E}_{a}$ die Größe der Energie-Barriere zwischen dem amorphen und dem kristallinen Zustand, die auch als Aktivierungsenergie für einen metastabilen Zwischenzustand verstanden werden kann.

Beim Prozess der Rekristallisation einer amorphen Schicht wird allgemein zwischen „Random Nucleation Growth" (RNG) und ,Solid Phase Epitaxial Growth“ (SPEG) unterschieden. Beim RNG-Prozess führt die größere Mobilität der Atome durch Temperaturerhöhung zur Bildung von Kristallisationskeimen und einer Zunahme der Nahordnung. Durch ein bevorzugtes Wachstum von Keimen mit größerer Korngröße auf Kosten kleinerer Agglomerate entsteht durch den RNG-Prozess ein polykristallines Material mit zufälliger Ausrichtung der einzelnen Kristallite. Für die epitaktische Rekristallisation (SPEG) hingegen ist ein einkristallines Substrat nötig und die Umwandlung von der amorphen Schicht zum Einkristall erfolgt schichtweise. 
In der vorliegenden Arbeit wird unter anderem die Laserbestrahlung von amorphen Deckschichten untersucht. Wie bereits im vorigen Abschnitt beschrieben, wird die Materialoberfläche durch die Laserstrahlung kurzzeitig erhitzt und dann abgeschreckt. Die erhitzte Oberfläche zeichnet sich durch eine große Mobilität der Atome aus. Dabei muss zunächst unterschieden werden, ob es durch die Laserbestrahlung lediglich zur Temperaturerhöhung der Oberfläche oder zum Aufschmelzen kommt.

Bei der thermischen Rekristallisation einer amorphen Schicht in der festen Phase wird eine gleichmäßige Bewegung der Rekristallisationsfront zur Oberfläche beobachtet (,SPEG“, s. Gl.32) [92, 93. Im Gegensatz dazu findet bei der laserinduzierten Rekristallisation der atomare Umordnungsprozess innerhalb der gesamten amorphen Schicht statt. Es existiert ein Schwellenwert für die Laserfluenz $\mathrm{F}_{r e c}\left(\mathrm{z}_{a m}\right)$, oberhalb derer für eine gegebene Dicke $\mathrm{z}_{a m}$ der amorphen Schicht vollständige epitaktische Rekristallisation auftritt. Dieser Schwellenwert lässt sich leicht verstehen unter der Annahme, dass es sich bei der lasergestützten Rekristallisation um Aufschmelzen mit darauf folgender epitaktischer Rekristallisation aus der flüssigen Phase handelt (Liquid Phase Epitaxial Growth, „LPEG“'). Dabei muss nicht nur die amorphe Schicht aufgeschmolzen werden, sondern die Schmelzfront muss auch die Grenze zur einkristallinen Phase überschreiten, um ein epitaktisches Schichtwachstum zu ermöglichen [94. Der Schwellenwert $\mathrm{F}_{r e c}\left(\mathrm{z}_{a m}\right)$ hängt ebenfalls von der Struktur der geschädigten Schicht ab. Nur wenig geschädigte oder polykristalline Schichten haben infolge des geringeren Absorptionskoeffizienten und der größeren Wärmekapazität höhere Werte von $\mathrm{F}_{r e c}$. Außerdem unterscheiden sich die Werte von $\mathrm{F}_{r e c}$ für $<100>-\mathrm{Si}$ und $<111>$-Si. Dies liegt an den unterschiedlichen Maximalgeschwindigkeiten für defektfreie Kristallisation $\mathrm{v}_{a / c}(\mathrm{Si}<100>) \leq 18 \mathrm{~m} / \mathrm{s}$ gegenüber $\mathrm{v}_{a / c}(\mathrm{Si}<111>) \leq 6 \mathrm{~m} / \mathrm{s}[95]$.

Der Übergang amorph-einkristallin erscheint bei der Untersuchung der Laser-Rekristallisation mittels RBS-C nicht abrupt, vielmehr nimmt die Kristallinität durch Laserbestrahlung scheinbar schrittweise zu. Dieser Effekt wird einem inhomogenen Laserprofil zugeschrieben [96]. Die lokale Laserfluenz ändert sich dadurch mit der Position auf der bestrahlten Schicht und kann in einigen Bereichen den Schwellenwert zur Rekristallisation schon überschritten haben. Da die Kristallinität der Proben nach der Laserbehandlung oft mittels RBS-Channeling (laterale Aufösung im mm-Bereich) bestimmt wird, kommt es zur Mittelung über bereits rekristallisierte und noch amorphe Bereiche der Probe. Foti et al. 97] finden für die Rekristallisation einer $450 \mathrm{~nm}$ dicken a-Si-Schicht auf einkristallinem Si $(<100>)$ durch Bestrahlung mit einem Rubin-Laser den Wert $\mathrm{F}_{r e c}=2 \mathrm{~J} / \mathrm{cm}^{2}$ als Schwelle für die vollständige epitaktische Rekristallisation des Films.

Schon bei sehr kleinen Laserfluenzen wird der Prozess der „explosiven Rekristallisation“ beobachtet. Dieser Prozess setzt ein, wenn die Oberflächentemperatur den Schmelzpunkt überschreitet und eine dünne Oberflächenschicht aufgeschmolzen wird. Die latente Wärme, die durch die Erstarrung der dünnen Schicht frei wird, ist höher als die Schmelztemperatur für a-Si. Daher schmilzt das darunter liegende amorphe Si auf. Diese Schmelze ist nun jedoch gegenüber dem neu gebildeten poly-Si stark unterkühlt und erstarrt sehr schnell zu poly-Si mit kleiner Korngröße. Die explosive Kristallisation von a-Si:H in einer dünnen Oberflächen-Schicht wurde bereits bei einer Laserfluenz von $\mathrm{E}_{L}=100 \mathrm{~mJ} / \mathrm{cm}^{2}$ beobachtet 98 .

Für die Laserrekristallisation von Silizium zur Herstellung elektronischer Bauelemente ist der Wasserstoffgehalt des Ausgangsmaterials in zweifacher Hinsicht wichtig: Wenn a-Si:H 
durch Laserbestrahlung aufschmilzt, zerfällt es in Wasserstoffgas und Silizium. Bei einem hohen Wasserstoffgehalt der Schicht kommt es durch den hohen Gasdruck zur Ablation. Daher ist ein niedriger H-Gehalt für Laser-Kristallisation besser geeignet. Andererseits ist ein hoher Wasserstoffgehalt nach der Rekristallisation der Schicht erwünscht, da Wasserstoff der Passivierung von Defekten an Korngrenzen bei polykristallinem Material dient.

\subsection{Wasserstoff-Diffusion}

In Kapitel 4.2 wurde die Wasserstoff-Diffusion in der flüssigen Phase des Siliziums als wichtigster Prozess für den Wasserstoffeintrag beim Laserhydrieren beschrieben. An dieser Stelle soll die Diffusion von Wasserstoff kurz skizziert werden.

Durch die zunehmende Verwendung von kristallinem und amorphen Silizium in der Halbleiterindustrie wurde in den 70er Jahren auch der Einfluss des bei der Bearbeitung von $\mathrm{Si}$ meist ungewollt eingebrachten Wasserstoffs untersucht. Ein erstes Ergebnis war die Feststellung, dass der Transportprozess der H-Atome stark von Einfangzentren (Fremdatomen und Defektstellen im Kristallgitter) abhängt. Dies drückt sich in höheren Aktivierungsenergien und damit kleineren Diffusionskoeffizienten aus [99]. Fremdatome können

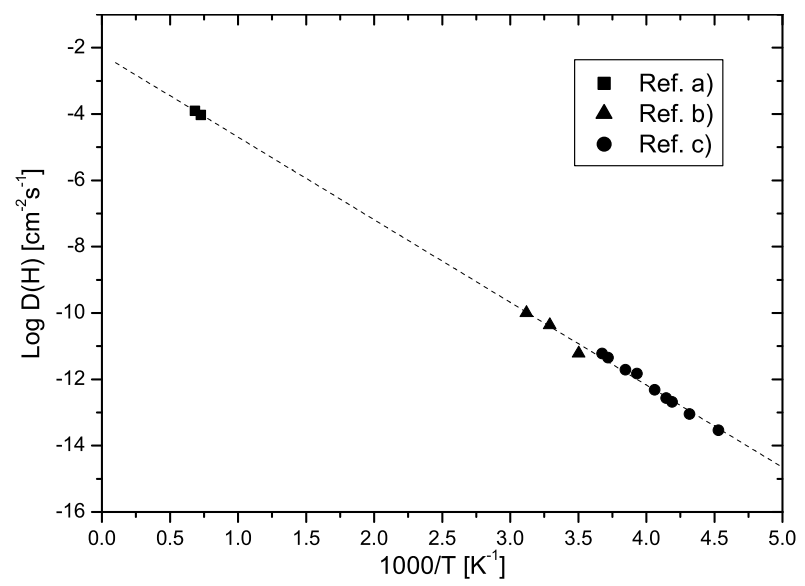

Abbildung 36: Literaturwerte für die Diffusionskonstante $D(H)$ von Wasserstoff in kristallinem Si. Für die Aktivierungsenergie ergibt sich der Wert $E_{a}=0.48 \mathrm{eV}$ (Ref. a): [100], Ref. b): [101], Ref. c): [99]).

in Abhängigkeit von ihrer Größe substitutionell oder interstitiell in das Gitter eines Festkörpers eingebaut werden. Die Diffusion der Fremdatome im Gitter hängt ab von der Potenzialtiefe an einem bestimmten Ort des Wirtsgitters und der Temperatur.

Die Bewegungen der Fremdatome können als Wechselwirkung mit den Gitterschwingungen des Kristalls, d.h. als Absorptions- und Reemissionsprozesse von Phononen verstanden werden. Es wird zwischen Schwingungen auf dem festen Platz und schnellen Platzwechseln (Sprünge) unterschieden. Die Energie der Phononen ist gequantelt und besitzt eine obere Schranke. Die maximale Schwingungsfrequenz definiert die Debye-Temperatur $\Theta_{D e b}$, mit deren Hilfe sich die verschiedenen Transportprozesse unterscheiden lassen. Für Temperaturen $T \gg \Theta_{D e b}$ dominiert ein thermisch aktivierter Prozess und es gilt die klassische 
Arrhenius-Beziehung (s. Abb. 36):

$$
D(T)=D_{0} \cdot e^{\frac{-E_{a}}{k_{b} T}}
$$

Die Debye-Temperatur von Silizium $\Theta_{D e b}(\mathrm{Si})=645 \mathrm{~K}$ wird bei den hier untersuchten Laserbestrahlungen stets überschritten.

Andererseits werden insbesondere bei der Laser-Materialbehandlung zum Teil sehr viele Defekte (z.B. Leerstellen und Zwischengitteratome) in das Kristallgitter eingebracht. Hierdurch können lokal zusätzliche und tiefere Potenziale auftreten. Die Störstellen bewirken ebenfalls eine räumliche Deformation des Gitters, wodurch eine für den Diffusor anziehende Kraft entsteht (Diffusionsfalle). Das Elektron des Wasserstoffatoms kann nun das durch die Defektstelle verursachte „dangling bond“ absättigen. Im Falle einer Leerstelle im Siliziumgitter entstehen sogar vier offene Bindungen, deren Absättigung durch Wasserstoffatome energetisch günstiger ist [102]. Damit ein Wasserstoffatom die „Falle" wieder verlassen kann, muss zusätzlich zur Diffusionsenergie die bei der Bindung des H-Atoms frei gewordene Energie aufgebracht werden („fallenbeeinflusste Diffusion“). 


\section{Probenpräparation}

Ein Teil der untersuchten Proben wurde durch Laserbestrahlung in Stickstoff- bzw. Wasserstoff-Atmosphäre hergestellt. Zusätzlich wurden einige Proben mittels Wasserstoff-Implantation am Niederenergie-Beschleuniger IOSCHKA und am Göttinger Schwerionenimplantator IONAS hergestellt.

Ausgangsmaterial für die Titanproben waren $2 \mathrm{~mm}$ dicke hochreine Titanplatten (99.999\%) der Größe $10 \times 13 \mathrm{~mm}$. Bei dem hier benutzten Silizium handelt es sich um phosphordotiertes (n-Typ) $\mathrm{Si}<100>$-Wafermaterial der Dicke $0.5 \mathrm{~mm}$.

\subsection{Excimer-Laser}

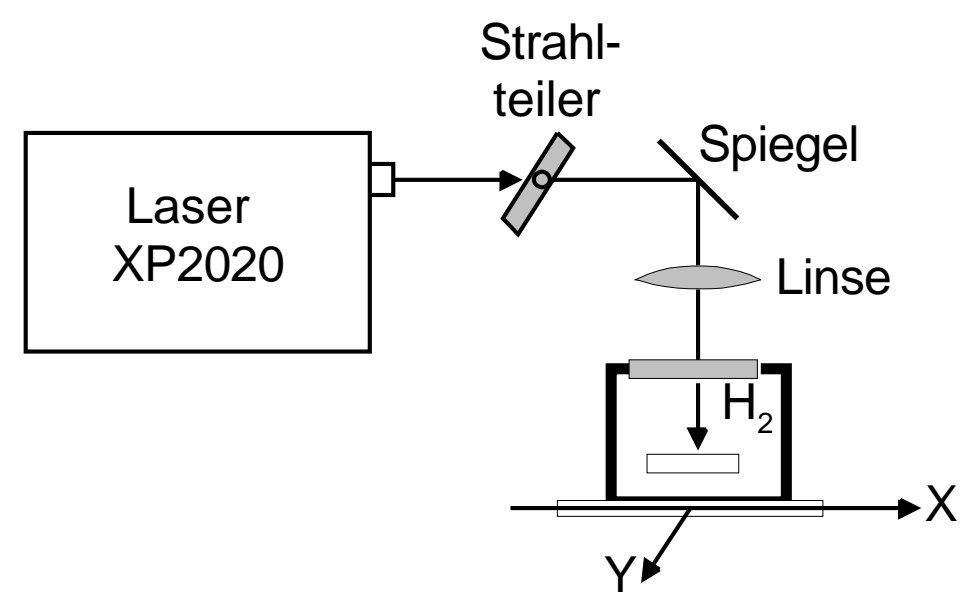

Abbildung 37: Strahlengang beim Laserhydrieren

Alle Laserbestrahlungen wurden mit einem Siemens XP2020-Excimerlaser im II. Physikalischen Institut der Universität Göttingen durchgeführt. Der Laser wird mit XeCl als Lasergas betrieben. Die Laserpulse haben eine Pulsdauer von $\tau=55$ ns bei einer Wellenlänge von $\lambda=308 \mathrm{~nm}$. Die Photonenenergie beträgt damit $\mathrm{E}_{\text {phot }}=4.025 \mathrm{eV}$. Für die in dieser Arbeit durchgeführten Bestrahlungen wurde durchweg der inhomogene Rohstrahl des Lasers verwendet, da der Einsatz des Homogenisators eine starke Einbuße an Laserintensität zur Folge hat. Um einen möglichst großen Bereich der Laserenergiedichten überdecken zu können, wurde die starke Inhomogenität des Laserprofils in Kauf genommen. Die maximal erreichbare Energiedichte beträgt bei Verwendung des Rohstrahls $\mathrm{F}=6.5 \mathrm{~J} / \mathrm{cm}^{2}$. In Abb. 37 ist der Versuchsaufbau zum Laserhydrieren dargestellt. Der Strahl des ExcimerLasers passiert zunächst einen Strahlteiler, der es durch Verkippen erlaubt, die transmittierte Strahlintensität auf den gewünschten Wert herabzusetzen. Anschließend trifft der Strahl auf einen Spiegel und wird durch die Linse auf die Probenoberfläche fokussiert. Zur Bestimmung der Energiedichte wurde vor jeder Bestrahlung ein thermoelektrischer Leistungsmesser der Fa. Gentec an die Stelle der Probenkammer eingesetzt. Für die mäanderförmige Bestrahlung der Proben (s. Abb. 38) wurde die Vakuumkammer mit den Proben auf einen X-Y-Tisch montiert, der mittels Schrittmotoren durch einen Computer verfahren werden konnte. 


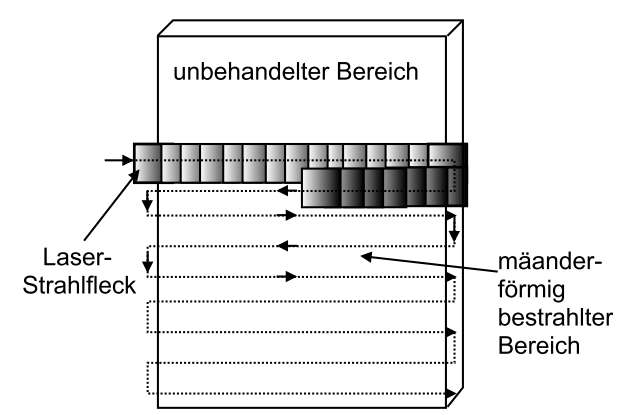

Abbildung 38: Skizze zum mäanderförmigen Überstreichen der Probe

Für eine detailierte Beschreibung des gesamten Aufbaus zur Bestrahlung von Proben im Vakuum oder in Gasatmosphäre sei hier auf [103, 3] verwiesen.

Die Proben wurden in einer Vakuumkammer belichtet, die nach dem Einbau der Proben auf ein Vakuum besser $10^{-3}$ Pa evakuiert wurde. Anschließend wurde die Kammer mit Wasserstoffgas der Reinheit $99.9993 \%$ bei Drücken zwischen $10^{5} \mathrm{~Pa}$ und $3 \times 10^{5} \mathrm{~Pa}$ gefüllt.

\subsection{Ionenimplantation}

Zur Ionenimplantation stehen im II. Physikalischen Institut der Universität Göttingen mittlerweile fünf Beschleuniger zur Verfügung (IOSCHKA, Mr. Stringer, ADONIS, IONAS und MaRPel), die Ionenstrahlen im Energiebereich $10 \mathrm{eV}$ bis $9 \mathrm{MeV}$ ermöglichen. Die Wasserstoff- und Xenon-Implantationen der hier untersuchten Proben wurden an drei verschiedenen Beschleunigern durchgeführt. Die folgenden Abschnitte beschreiben die wichtigsten Bestrahlungsparameter.

\section{IOSCHKA}

Bei IOSCHKA („Ionen Schleuder-Kammer“) handelt es sich um einen NiederenergieImplanter, der zeitlich sehr konstante Ionenstrahlen im Energiebereich von $10 \mathrm{eV}$ bis $10 \mathrm{keV}$ liefert [104, 105]. Die Wasserstoff-Implantationen erfolgten hier stets bei Raumtemperatur. Es wurden Siliziumproben mit verschiedenen Fluenzen zwischen $1 \times 10^{15}$ und $1 \times 10^{16} \mathrm{H} / \mathrm{cm}^{2}$ bei Strahlströmen von ca. $100 \mathrm{nA}$ bestrahlt, die später als Eichproben für die NRA-Methode verwendet wurden.

\footnotetext{
ADONIS

Die Wasserstoff-Implantationen an ADONIS [106] wurden ebenfalls bei Raumtemperatur durchgeführt. ADONIS bietet gegenüber IOSCHKA höhere Ionenergien $(\mathrm{E} \leq 30 \mathrm{keV})$ und vor allem wesentlich höhere Strahlströme $\left(\mathrm{I}_{\text {target }} \leq 6 \mu \mathrm{A}\right)$. Deswegen wurden an ADONIS die sonst zeitintensiven Hochdosis-Bestrahlungen im Bereich $5 \times 10^{16}-2 \times 10^{17} \mathrm{H} / \mathrm{cm}^{2}$ durchgeführt. An ADONIS wurde die Ionenenergie auf $30 \mathrm{keV}$ eingestellt. Da jedoch molekularer Wasserstoff implantiert wurde, ergibt sich daraus eine Energie von $15 \mathrm{keV}$ je Wasserstoffion. Auch diese Proben wurden später zur Kalibration der Wasserstoff-Konzentration genutzt.
} 


\section{IONAS}

Weitaus die meisten Wasserstoff-Implantationen sowie alle Xenon-Bestrahlungen wurden am Strahlrohr L30 des Göttinger Schwerionenimplantators IONAS [2] durchgeführt. Bei allen Implantationen am IONAS wurden die Targets auf Stickstoff-Temperatur herunter gekühlt [61]. Bei den Wasserstoff-Implantationen wurde wie am ADONIS molekularer Wasserstoff mit $30 \mathrm{keV}$ im Fluenzbereich $5 \times 10^{12}-1 \times 10^{17} \mathrm{H} / \mathrm{cm}^{2}$ implantiert. Bei den Xenon-Implantationen betrug die Strahlenergie stets $450 \mathrm{keV}$. In allen Fällen lag der Strahlstrom bei ca. $3 \mu \mathrm{A}$.

\subsection{Probenübersicht}

Tabelle 7 gibt einen Überblick über die Präparationsparameter der verwendeten Titan und Siliziumproben. Außerdem ist in der letzten Spalte der Abschnitt der Arbeit angegeben, in dem die zugehörigen Ergebnisse diskutiert werden.

\begin{tabular}{c|c|c|c|c|r|} 
Material & Implantat & $\begin{array}{c}\text { Ionenfluenz } \phi \\
{\left[10^{16} \mathrm{ion} / \mathrm{cm}^{2}\right]}\end{array}$ & $\begin{array}{c}\text { Laserfluenz } \mathrm{F} \\
{\left[\mathrm{J} / \mathrm{cm}^{2}\right]}\end{array}$ & $\begin{array}{c}\text { H-Druck } \\
{[\mathrm{bar}]}\end{array}$ & \multicolumn{2}{|c}{ Abschnitt } \\
\hline \hline $\mathrm{Ti}$ & - & - & 4 & $0.5-3$ & 6.1 \\
\hline $\mathrm{c}-\mathrm{Si}$ & - & - & $\sim 3-6.3$ & 1,3 & 6.2 \\
\hline $\mathrm{a}-\mathrm{Si}$ & $450 \mathrm{keV} \mathrm{Xe}$ & $0.5,1$ & $0.5-5$ & 2 & 6.3 .1 \\
\hline $\mathrm{a}-\mathrm{Si}$ & $30 \mathrm{keV} \mathrm{H}$ & $5,6,10,15$ & $0.5,1$ & 2 & 6.3 .2 \\
\hline
\end{tabular}

Tabelle \%: Herstellungsparameter der verwendeten Titan und Siliziumproben 


\section{Ergebnisse und Diskussion}

Im Folgenden werden die Ergebnisse zum Laserhydrieren von Titan, kristallinem Silizium (c-Si) und amorphem Silizium (a-Si) dargestellt. Am Beispiel von Titan, das für seine Fähigkeit zur Speicherung großer Mengen Wasserstoff bekannt ist, konnte untersucht werden, ob das Laserhydrieren ähnlich zum Lasernitrieren abläuft. Mit kristallinem und amorphem Silizium wurde dann untersucht, ob es durch Laserhydrieren gelingt, eine größere Menge Wasserstoff in Silizium einzubringen. Dabei wurde die Entwicklung der Oberflächenrauigkeit und die Bildung von Defekten und ihre Rekristallisation studiert. Der Vergleich mit Simulationsrechnungen erlaubt Rückschlüsse auf den Einfluss der Probentemperatur auf den Wasserstoffgehalt.

\subsection{Laserhydrieren von Titan}

Die Messungen zum Laserhydrieren von Titan stellen den ersten Test dar, ob es bei der Laserbestrahlung von Titan in Wasserstoffatmosphäre zu ähnlichen Effekten (z.B. Wasserstoffeintrag) kommt, wie bei dem bereits ausführlich behandelten Lasernitrieren [3]. Hierbei wurden insbesondere die Wasserstoffaufnahme nach dem Laserhydrieren und die laserinduzierte Phasenbildung der Proben untersucht.

Beim Lasernitrieren von Titan war gezeigt [107, 108] worden, dass die Effizienz des Nitrierprozesses (d.h. die Menge des eingebrachten Stickstoffs und die Dicke der modifizierten Schicht) gegeben ist durch das Verhältnis der durch Diffusion eingebrachten Stickstoffmenge zum Stickstoffverlust durch Ausgasen an der Materialoberfläche beim Abkühlen des Materials. Die Untersuchungen hatten ergeben, dass lasernitrierte Titanproben, die ebenfalls mit dem auch in dieser Arbeit verwendeteten Excimer-Laser bei der Laserfluenz $\mathrm{F}=4 \mathrm{~J} / \mathrm{cm}^{2}$ und einem Stickstoffdruck von $\mathrm{p}=2$ bar bestrahlt wurden, eine konstante Stickstoffkonzentration von $\mathrm{c}(\mathrm{N}) \approx 50$ at\% bis in eine Tiefe von $370 \mathrm{~nm}$ aufweisen. Eine Abhängigkeit der Nitriereffizienz von der Wellenlänge des Lasers wurde nicht festgestellt, sofern die Laserfluenz zum Zünden eines Plasma ausreicht [107].

Abbildung 39 zeigt Wasserstofftiefenprofile von Titanproben, die mit 16 Laserpulsen (oben), 64 Laserpulsen (mitte) und 256 Laserpulsen (unten) bei der festen Laserfluenz $\mathrm{F}=4 \mathrm{~J} / \mathrm{cm}^{2}$ und bei Wasserstoffdrücken von $\mathrm{p}=0.5,1,2$ und 3 bar laserhydriert wurden. Die gezeigten Proben wurden vom Laserstrahl mäanderförmig mit einer Überdeckung von 25\% überstrichen (s. Abb. 38, S. 57). Alle Proben zeigen ein Ansteigen der Wasserstoffkonzentration im oberflächennahen Bereich auf 30 at\% innerhalb von 20-50 nm und danach einen verlangsamten Anstieg in eine Sättigung. Die Proben, deren Wasserstoffkonzentration bis in eine Tiefe von $370 \mathrm{~nm}$ gemessen wurde, zeigen übereinstimmend eine maximale Wasserstoffkonzentration von ca. 47 at\%. 


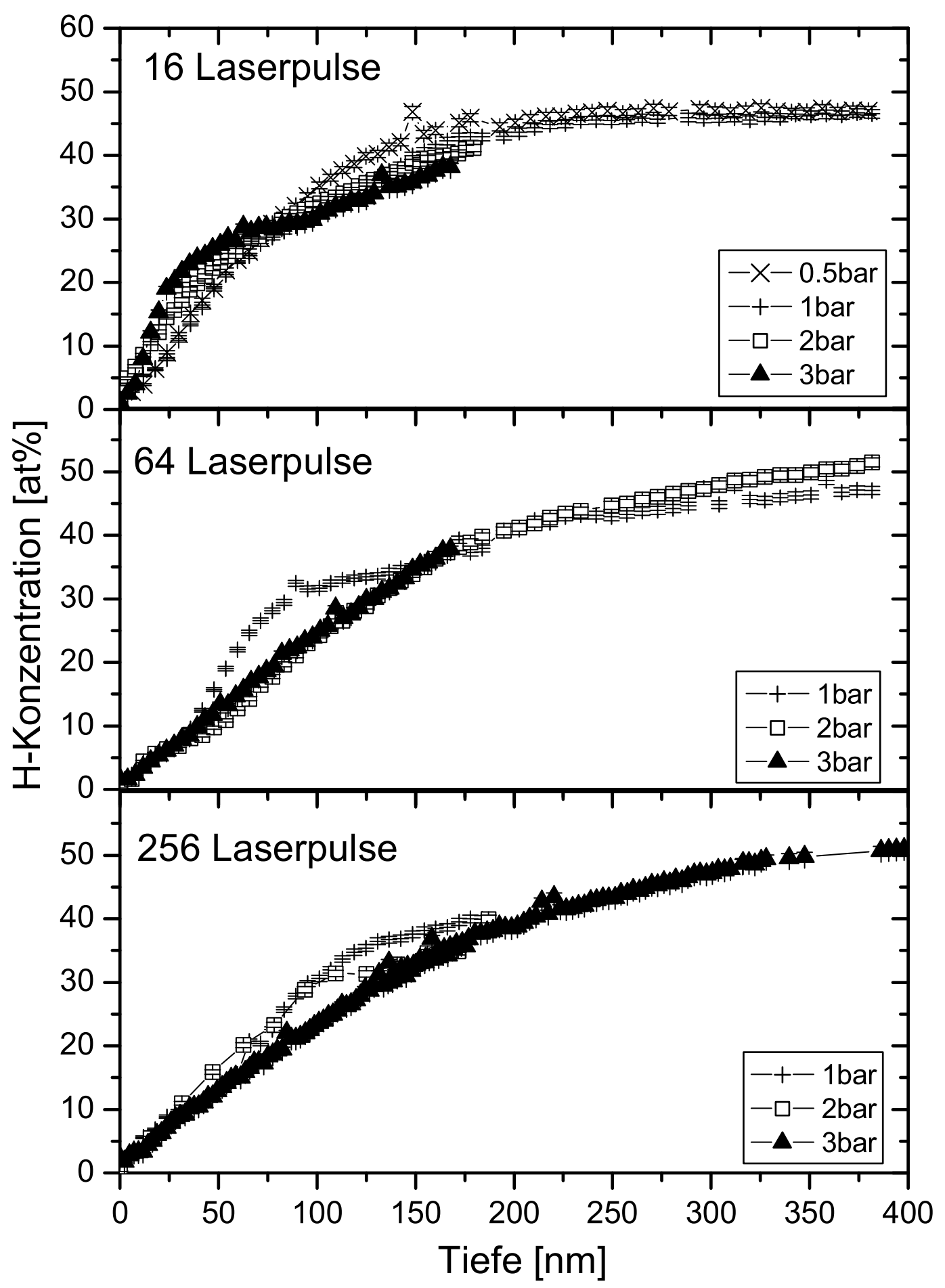

Abbildung 39: Wasserstofftiefenprofile von Titanproben, die mit der Laserfluenz $F=4 \mathrm{~J} / \mathrm{cm}^{2}$ bei Drücken von $p=0.5,1,2$ und 3 bar laserhydriert wurden. Oben: 16 Laserpulse, Mitte: 64 Laserpulse, Unten: 256 Laserpulse 


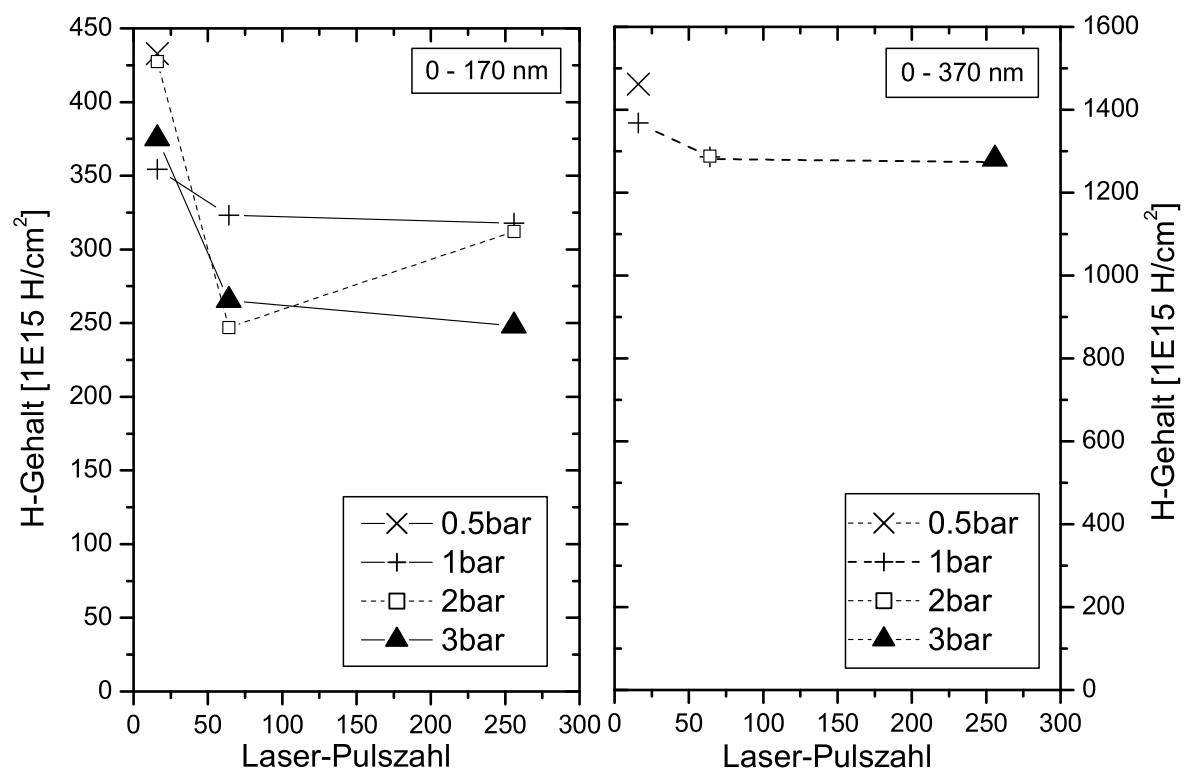

Abbildung 40: Integraler H-Gehalt von laserhydrierten Titanproben als Funktion der Zahl der Laserpulse (Fluenz $F=4 \mathrm{~J} / \mathrm{cm}^{2}, p=0.5,1,2$ und $3 \mathrm{bar}$ ). Links: Integraler H-Gehalt im oberflächennahen Bereich bis $170 \mathrm{~nm}$. Rechts: Wasserstoffgehalt im gesamten gemessenen Tiefenbereich bis $370 \mathrm{~nm}$. Der Abfall des H-Gehalts beim Übergang von 16 auf 64 Laserpulse ist wegen des geringen Fehlers von $\leq 56 \times 10^{15} \mathrm{H} / \mathrm{cm}^{2}$ in beiden Teilen der Abbildung signifikant.

Alle Wasserstofftiefenprofile zeigen einen ähnlichen Verlauf, und erreichen eine Maximalkonzentration von ca. 50 at\% in einer Tiefe von 400 nm. Die Profile derjenigen Proben, die bei einem geringen Kammerdruck ( 0.5 bar bzw. 1 bar $\mathrm{H}_{2}$ ) bestrahlt wurden, zeigen jedoch einen schnelleren Anstieg der Wasserstoffkonzentration im Tiefenbereich zwischen 50 und $100 \mathrm{~nm}$. Als Erklärung hierfür könnte ein bei niedrigen Drücken verminderter „Kolbeneffekt" dienen (s. Abs. 4.2.

Der integrale Wasserstoffgehalt der laserhydrierten Titanproben ist in Abb. 40 in Abhängigkeit von der Laserpulszahl dargestellt. Dabei zeigt die linke Auftragung den integralen Wasserstoffgehalt im oberflächennahen Bereich bis in eine Tiefe $\mathrm{z}_{i}=170 \mathrm{~nm}$ als Funktion der Laserpulszahl und rechts ist der Wasserstoffgehalt für den gesamten gemessenen Bereich bis $\mathrm{z}_{\max }=370 \mathrm{~nm}$ ebenfalls als Funktion der Laserpulse dargestellt. Der Wasserstoffgehalt der Proben zeigt einheitlich den Trend, dass eine niedrige Zahl von Laserpulsen zu einer höheren Wasserstoffaufnahme führt. Sowohl bei der Betrachtung des oberflächennahen Bereichs, als auch bei dem integralen Wasserstoffgehalt bis $\mathrm{z}_{\max }=370 \mathrm{~nm}$ sinkt der Wasserstoffgehalt bei einer Erhöhung der Laserpulszahl von 16 auf 64 Pulse. Eine weitere Erhöhung der Pulszahl zeigt dagegen keine Auswirkung auf den Wasserstoffgehalt. Nach Abb. 40 ist die aufgenommene Wasserstoffmenge unabhängig von dem Kammerdruck während des Laserhydrierens. Die bei dem niedrigsten Kammerdruck (0.5 bar) und niedrigster Laserpulszahl (16 Pulse) laserhydrierte Titanprobe zeigt den höchsten Wasserstoffgehalt.

Die Beschränkung der Messung auf eine Tiefe von $\mathrm{z}_{\max }=370 \mathrm{~nm}$ ist durch die maximal erreichbare Energie $\mathrm{E}=7.2 \mathrm{MeV}$ des ${ }^{15} \mathrm{~N}^{2+}$-Analysestrahls begründet. Eine Untersuchung in größeren Tiefenbereichen wäre nur durch die Nutzung eines ${ }^{15} \mathrm{~N}^{3+}$-Analysestrahls möglich geworden, der jedoch nur mit einem erheblich geringeren Strahlstrom zur Verfügung 
stand $\left(\mathrm{I}_{\max }\left({ }^{15} \mathrm{~N}^{3+}\right) \approx 300 \mathrm{nA}\right.$, gegenüber $\left.\mathrm{I}_{\max }\left({ }^{15} \mathrm{~N}^{2+}\right) \approx 1.2 \mu \mathrm{A}\right)$.

Zum Test, ob es bei der NRA-Analyse des laserhydrierten Titans zu einer Veränderung des

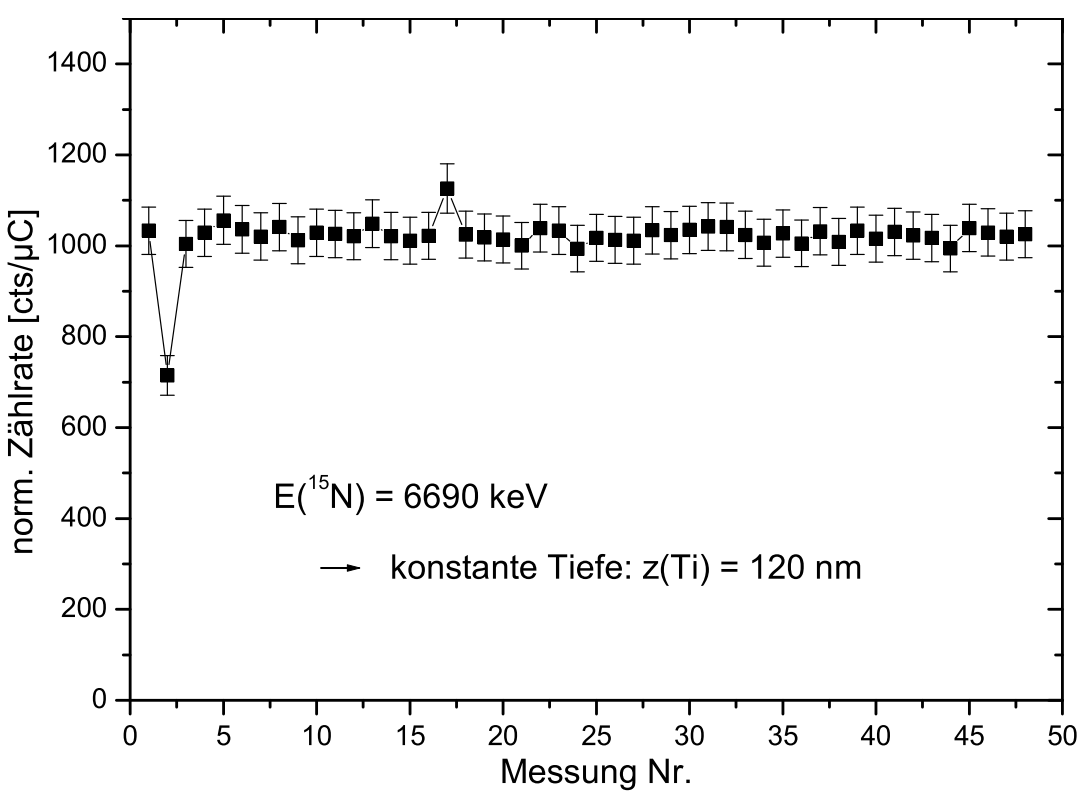

Abbildung 41: NRA-Messung einer laserhydrierten Titanprobe, die mit 256 Laserpulsen (Fluenz $F=4 \mathrm{~J} / \mathrm{cm}^{2}$ ) bei einem Druck von $p=2$ bar laserhydriert wurde. Bei dieser Messung wurde die Energie des ${ }^{15} \mathrm{~N}$-Analysestrahls konstant bei $E=6690 \mathrm{keV}$ gehalten. Dies entspricht einer Tiefe in Titan von $120 \mathrm{~nm}$.

Wasserstofftiefenprofils durch den ${ }^{15} \mathrm{~N}^{2+}$-Analysestrahl kommt, wurde eine Reihe NRASpektren bei konstanter Strahlenergie aufgenommen. Abbildung 41 zeigt die normierte Zählrate für 48 aufeinanderfolgende NRA-Messungen der gleichen Probe bei konstanter Energie des ${ }^{15} \mathrm{~N}^{2+}$-Analysestrahls von $\mathrm{E}=6690 \mathrm{keV}$. Diese Energie entspricht einer Tiefe von $\mathrm{z}(\mathrm{Ti})=120 \mathrm{~nm}$. Mit Ausnahme der Messungen Nr. 2 und Nr. 17 ist die normierte Zählrate sehr konstant, mit einem Mittelwert von $\overline{Y_{\text {norm }}}=1019(7)$ cts $/ \mu C$. Damit ist nachgewiesen, dass sich die Wasserstoffkonzentration von $\mathrm{c}_{H}(120 \mathrm{~nm})=30$ at\% durch den Analysestrahl während der Messung nicht ändert.

Die Untersuchungen zum Lasernitrieren hatten gezeigt, dass sich an der Titanoberfläche kristallines TiN bildet [107]. Für das hier untersuchte Laserhydrieren von Titan lässt das Ti-H-Phasendiagramm (s. Anhang F.1) die Hypothese zu, dass sich durch das Laserhydrieren unterhalb der Oberfläche eine Titandihydrid-Phase $\left(\mathrm{TiH}_{2}\right)$ bildet.

Zur Überprüfung wurden an zwei der laserhydrierten Proben XRD-Messungen durchgeführt. Das Ergebnis ist in Abb. 42 dargestellt. Die durchgezogene Linie zeigt das XRDSpektrum des unbehandelten hexagonalen Titans, deren Lage exakt mit den in [109] angegebenen Werten übereinstimmt.

Zusätzlich zeigt Abb. 42 die XRD-Spektren einer bei 0.5 bar (ausgefüllte Kreise) und einer bei 2 bar (offene Kreise) laserhydrierten Probe. Die indizierten charakteristischen Reflexe der $\mathrm{TiH}_{2}$-Phase entsprechen den in Tabelle 8 angegebenen Werten. Zwar liegen die starken Reflexe für $\mathrm{Ti}(<100>)$ und $\mathrm{TiH}_{2}(<111>)$ sehr nahe beieinander, aber die Reflexe der $<220>$-Ebene $\left(58.7^{\circ}\right)$, sowie der $<222>$-Ebene $\left(73.7^{\circ}\right)$ des $\mathrm{TiH}_{2}$ sind eindeutige Indizien für die Entstehung von Titandihydrid beim Laserhydrieren (s. Vergrößerung in Abb. 43). 


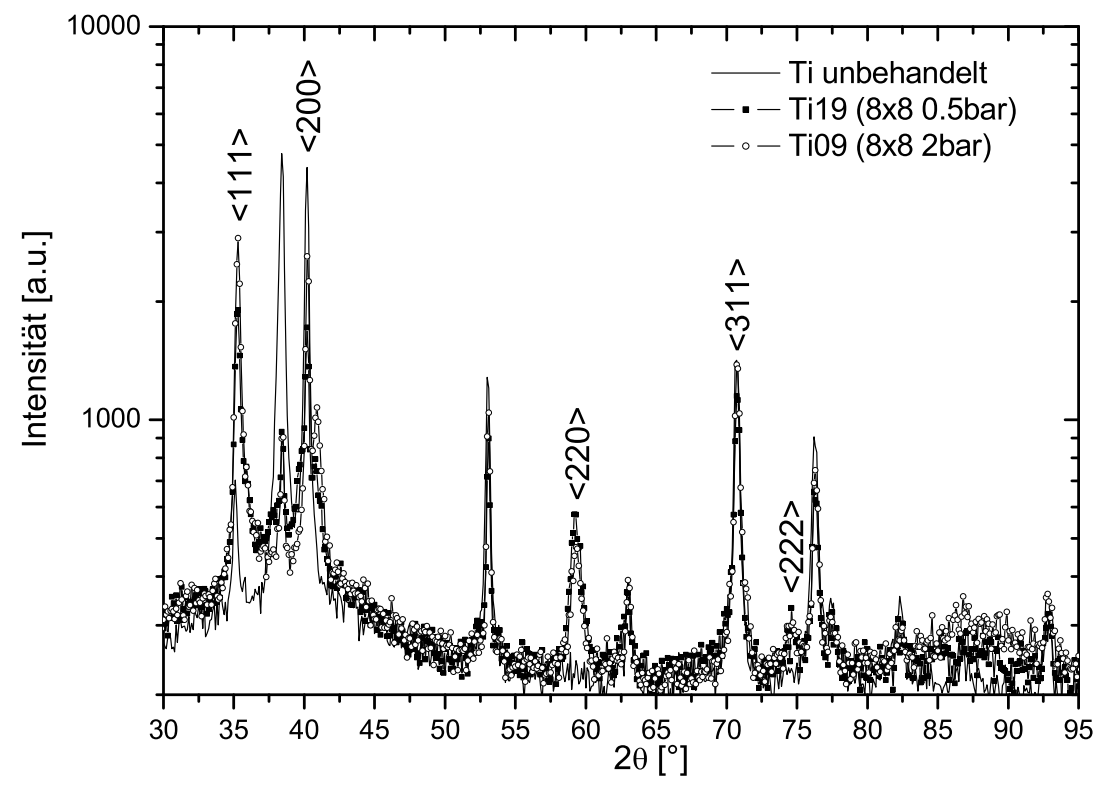

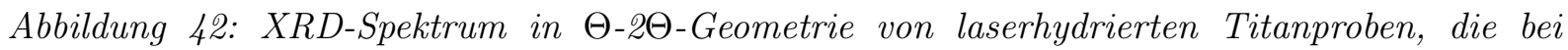
0.5 bar und 2 bar mit 64 Pulsen laserhydriert wurden (Symbole). Zum Vergleich ist ein XRDSpektrum einer unbehandelten Probe (Linie) mit angegeben. Die Indizes der charakteristischen Reflexe der $\mathrm{TiH}_{2}$-Phase sind eingezeichnet.
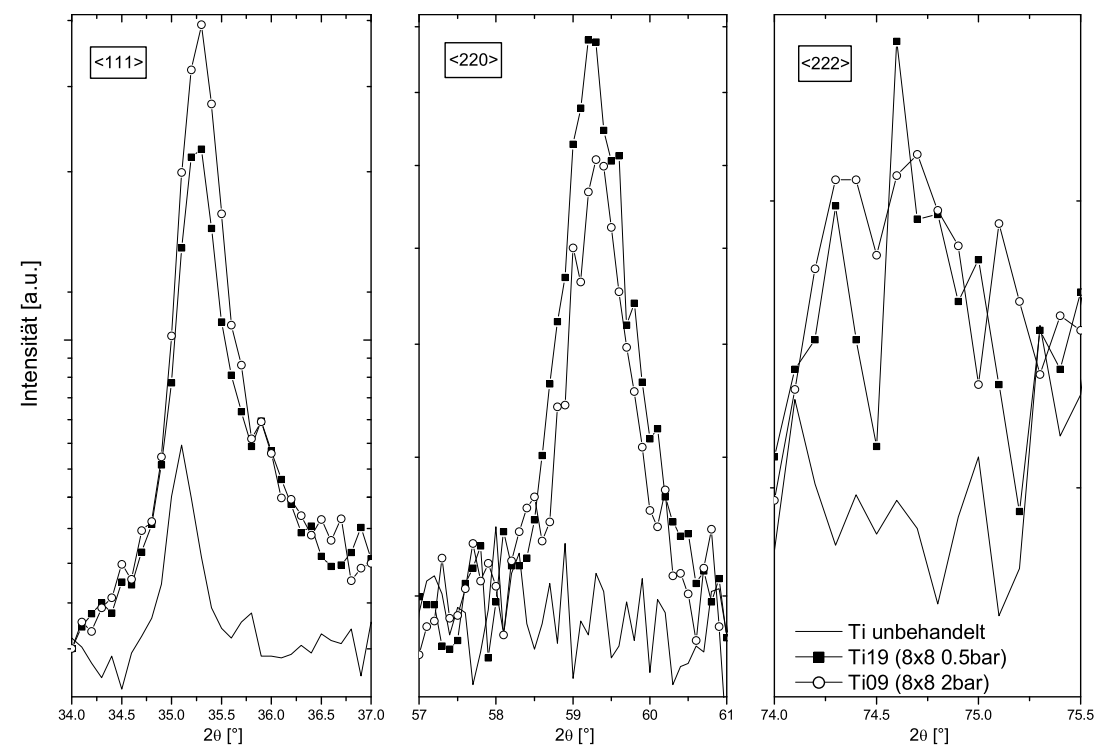

Abbildung 43: Vergrößerung von Abb. 42. Die Teilbilder zeigen die Röntgenreflexe für die $<111>-$, $<200>$ - und die <220>-Gitterebenen des $\mathrm{TiH}_{2}$ 


\begin{tabular}{c|c|c|c|c||c|c|c|c|c}
\multicolumn{5}{c||}{ Titan } & \multicolumn{9}{c||}{$\mathrm{TiH}_{2}$} \\
\hline $2 \theta$ & Intensität & $\mathrm{h}$ & $\mathrm{k}$ & $\mathrm{l}$ & $2 \theta$ & Intensität & $\mathrm{h}$ & $\mathrm{k}$ & $\mathrm{l}$ \\
\hline \hline 35.092 & 25 & 1 & 0 & 0 & 34.929 & 100 & 1 & 1 & 1 \\
\hline 38.419 & 30 & 0 & 0 & 2 & 40.568 & 25 & 2 & 0 & 0 \\
\hline 40.169 & 100 & 1 & 0 & 1 & 58.70 & 35 & 2 & 2 & 0 \\
\hline 53.002 & 13 & 1 & 0 & 2 & 70.143 & 25 & 3 & 1 & 1 \\
\hline 62.946 & 11 & 1 & 1 & 0 & 73.733 & 8 & 2 & 2 & 2 \\
\hline 70.657 & 11 & 1 & 0 & 3 & - & - & - & - & - \\
\hline 76.215 & 9 & 1 & 1 & 2 & - & - & - & - & - \\
\hline
\end{tabular}

Tabelle 8: Charakteristische Röntgenreflexe und zugehörige Intensitäten und Kristallebenen für $\mathrm{Ti}$ (aus [109]) und $\mathrm{TiH}_{2}$ (aus [110])

In Abbildung 44 ist ein GI-XRD-Spektrum einer laserhydrierten Titanprobe dargestellt, das bei streifendem Einfall unter $1^{\circ}$ gemessen wurde (GI-XRD, ,Glancing Incidence XRay Diffraction"). Die Abbildung zeigt eine Verschiebung der Peaks der gemessenen Probe gegenüber den Literaturwerten der kubischen TiH-Phase mit der Stöchiometry $\mathrm{TiH}_{1.924}$ [110]. Wird die Gitterkonstante $\mathrm{a}=4.448 \mathrm{des}^{\mathrm{TiH}_{1.924}}$ auf $\mathrm{a}_{\text {fit }}=4.41(2)$ verringert, so ergibt sich eine gute Übereinstimmung mit dem in Abb. 44 gezeigten GI-XRD-Spektrum. Dies erlaubt eine Abschätzung für die Menge des im TiH gelösten Wasserstoffs (s. Tab. 9). Mit der Näherung eines linearen Zusammenhangs zwischen Gitterparameter und Wasser-

\begin{tabular}{c|c|c}
$\begin{array}{c}\text { Gitterkonstante a } \\
{[\AA]}\end{array}$ & $\begin{array}{c}\text { Wasserstoffanteil x } \\
{\left[\mathrm{TiH}_{x}\right]}\end{array}$ & Ref. \\
\hline \hline 4.440 & 1.971 & {$[110]$} \\
\hline 4.448 & 1.924 & {$[111]$} \\
\hline \hline $4.41(2)$ & $2.1(1)$ & Messung \\
\hline
\end{tabular}

Tabelle 9: Gitterkonstante a und Wasserstoffanteil x. Die letzte Zeile gibt die an das GI-XRDSpektrum (Abb. 44) angepasste Gitterkonstante $a_{f i t}$ und das Ergebnis der linearen Regression für den Wasserstoffgehalt $x$ wieder.

stoffgehalt ergibt sich für die in Abb. 44 gezeigte Probe, die mit 16 Laserpulsen bei 2 bar Wasserstoffdruck laserhydriert wurde, für den Wasserstoffanteil x innerhalb der vorliegenden $\mathrm{TiH}_{x}$-Phase zu x=2.1(1). Bei der GI-XRD-Messung unter $1^{\circ}$ ist die Eindringtiefe auf die obersten $400 \mathrm{~nm}$ der Titanprobe beschränkt. Folglich wurde in diesem Bereich durch das Laserhydrieren der dargestellten Probe Titanhydrid in der Zusammensetzung $\mathrm{TiH}_{2.1}$ gebildet.

Obwohl die Tiefenprofile in größeren Tiefen einen relativ hohen H-Gehalt zeigen, deuten die geringen Intensitäten der XRD-Messungen nur auf einen kleinen Anteil der $\mathrm{TiH}_{2}-$ Phase hin. Während das beim Lasernitrier-Prozess hauptsächlich entstehende TiN mit einem Schmelzpunkt von $\mathrm{T}_{\text {melt }}=3020 \mathrm{~K}$ thermisch sehr stabil ist, zerfällt $\mathrm{TiH}_{2}$ schon bei $\mathrm{T}=720 \mathrm{~K}$ in Titan und Wasserstoff.

Zusammenfassend haben die Untersuchungen gezeigt, dass durch das Laserhydrieren von Titan eine hohe Wasserstoffkonzentration von bis zu 47 at\% in einer Tiefe von $400 \mathrm{~nm}$ 


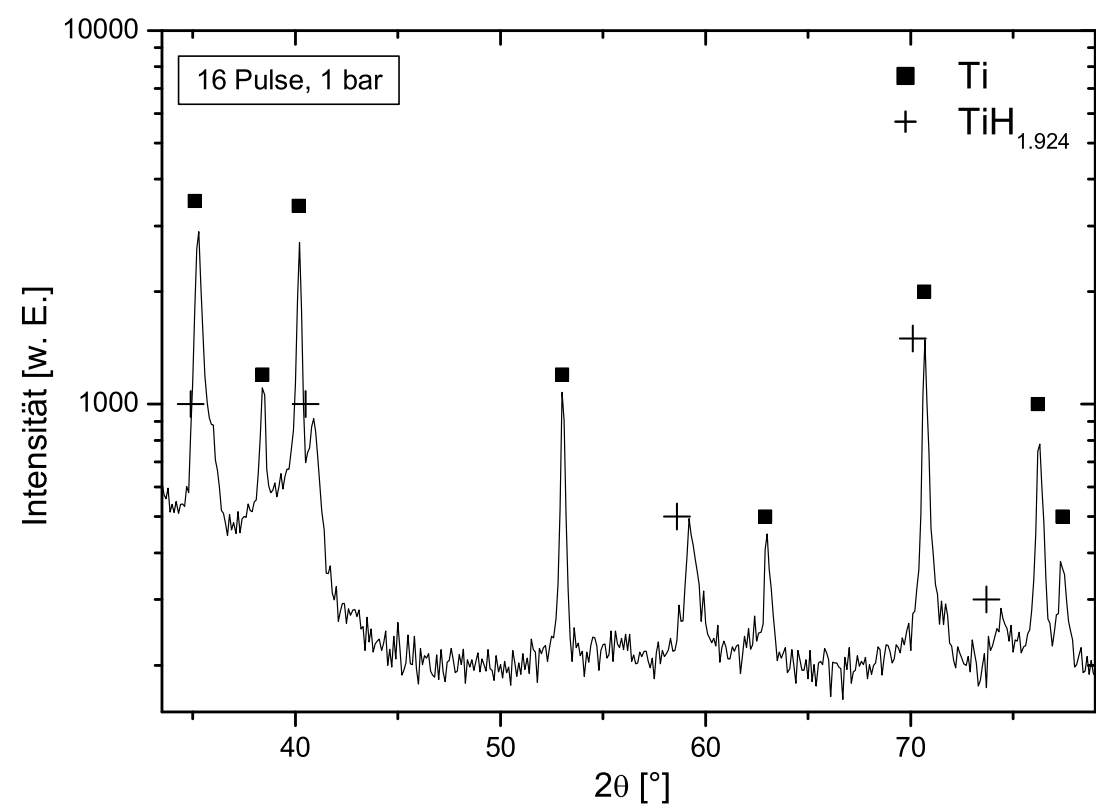

Abbildung 44: GI-XRD-Spektrum einer mit 16 Laserpulsen bei 1 bar Wasserstoffdruck laserhydrierten Titanprobe $\left(F=4 \mathrm{~J} / \mathrm{cm}^{2}\right)$. Die Quadrate markieren die Lage der Reflexe des Ti, die Kreuze markieren die Lage der unverschobenen TiH1.924-Reflexe [110]

erreicht wird. Dieser Grenzwert ist unabhängig sowohl von der Zahl der Laserpulse, als auch vom Wasserstoffdruck. Aus den XRD-Messungen folgt, dass der Wasserstoff in Form der Titanhydrid-Phase mit der Komposition $\mathrm{TiH}_{2.1}$ vorliegt.

\subsection{Laserhydrieren von kristallinem Silizium}

In diesem Abschnitt werden die Auswirkungen des Laserhydrierens auf den Wasserstoffgehalt, die Kristallstruktur und die Oberflächenmorphologie von kristallinem Silizium (c-Si) behandelt.

\section{Wasserstoffaufnahme}

Nach dem Laserhydrieren wurde die Wasserstofftiefenverteilung mittels der NRA-Methode mit MaRPel vermessen. Es zeigt sich, dass diejenige c-Si-Probe, die mit der höchsten Laserfluenz bestrahlt wurde, den höchsten Wasserstoffgehalt aufweist. Abbildung 45 gibt Wasserstofftiefenprofile von c-Si-Proben wieder, die mit jeweils 64 Laserpulsen bei einem Druck von 1 bar Wasserstoff mit verschiedenen Laserfluenzen laserhydriert wurden. Zusätzlich ist das Wasserstofftiefenprofil einer unbehandelten c-Si-Probe angegeben (offene Symbole). Diese Probe zeigt nur an der Oberfläche einen erhöhten Wasserstoffgehalt (5 at\%), wobei es sich um den stets vorhandenen „Oberflächenpeak" handelt, der von adsorbiertem Wasserdampf aus dem Restgas der Kammer herrührt. Auch die Wasserstoffprofile der laserhydrierten Proben weisen ein Maximum an der Probenoberfläche auf. Diese Maxima müssen ebenfalls auf adsorbierten Wasserdampf an der Probenoberfläche zurückgeführt werden. Abgesehen vom Wasserstoff-Oberflächen-Peak ist festzustellen, 


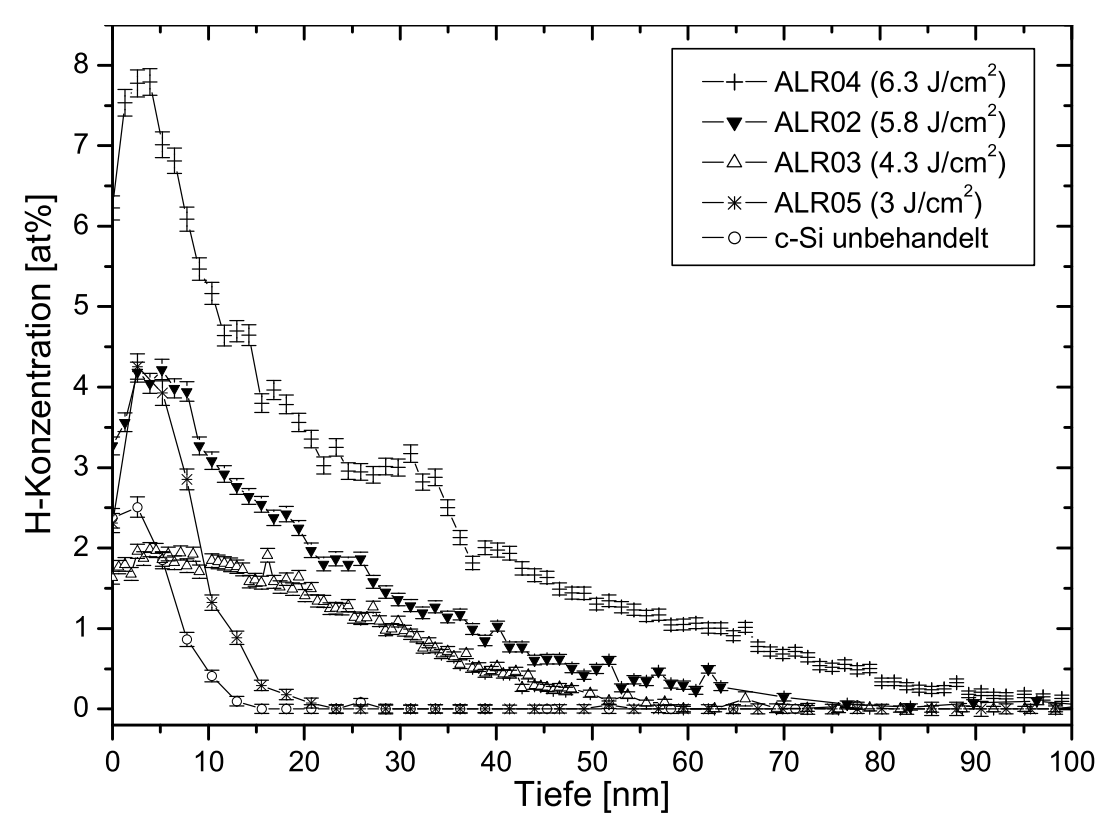

Abbildung 45: Wasserstofftiefenprofile von c-Si-Proben, die bei den angegebenen Laserfluenzen (64 Laserpulse, $p=1$ bar) laserhydriert wurden. Zusätzlich ist das Wasserstofftiefenprofil (Oberflächenpeak) einer unbehandelten Si-Probe angegeben (offene Kreise).

dass die Wasserstoffaufnahme nicht sehr effizient ist. Sie beträgt 4-8 at\% nahe der Oberfläche und vermindert sich auf die Hälfte innerhalb der ersten $30 \mathrm{~nm}$.

\section{Oberflächenrauigkeit}

Allgemein nimmt die Oberflächenrauigkeit der Proben durch das Laserhydrieren mit zunehmender Fluenz F des Laserstrahls zu. Die Veränderung der Oberflächenrauigkeit durch die Laserbehandlung ist in Abb. 46 deutlich sichtbar. Das obere Höhenprofil (A) zeigt die relativ glatte Oberfläche einer mit der Laserfluenz $\mathrm{F}=3 \mathrm{~J} / \mathrm{cm}^{2}$ bestrahlten Siliziumprobe. Das im unteren Teil gezeigte Höhenprofil (B) einer mit der Laserfluenz $\mathrm{F}=5 \mathrm{~J} / \mathrm{cm}^{2}$ bestrahlten Probe zeigt eine deutlich größere Rauigkeit.

Wie Abb. 47 zeigt, nimmt die Oberflächenrauigkeit allerdings erst oberhalb einer Fluenz von $\mathrm{F}=4 \mathrm{~J} / \mathrm{cm}^{2}$ stark zu. Dieser Wert markiert gleichzeitig die Schwelle, oberhalb derer beim Laserhydrieren ein Plasma an der Probenoberfläche zündet. Das bläulich gelbe Plasma wird bei der Bestrahlung von einem lauten Knall begleitet (vgl. Kap. 5.1).

Die Aufrauung der Probenoberfläche durch die Laserbestrahlung trägt zu einer effektiven Vergrößerung der Oberfläche und damit zu einer erhöhten adsorbierten Menge Wasserstoff bei. Die Rauigkeit einer unbehandelten c-Si-Probe von $\mathrm{R}_{a}=5.5(1) \mathrm{nm}$ ist ebenfalls in Abb. 47 eingezeichnet. Durch Kontrollmessungen konnte ausgeschlossen werden, dass die Richtung auf der Probenoberfläche, in der die Oberflächenrauigkeit gemessen wird, den Rauigkeitswert $\mathrm{R}_{a}$ beeinflusst. Der starke Anstieg der Oberflächenrauigkeit durch das Laserhydrieren mit hoher Laserfluenz $\mathrm{F} \geq 5 \mathrm{~J} / \mathrm{cm}^{2}$ sollte ebenfalls eine Zunahme der normierten Schädigung $\chi_{\text {norm }}$ nach sich ziehen, die im folgenden Abschnitt untersucht wird. 


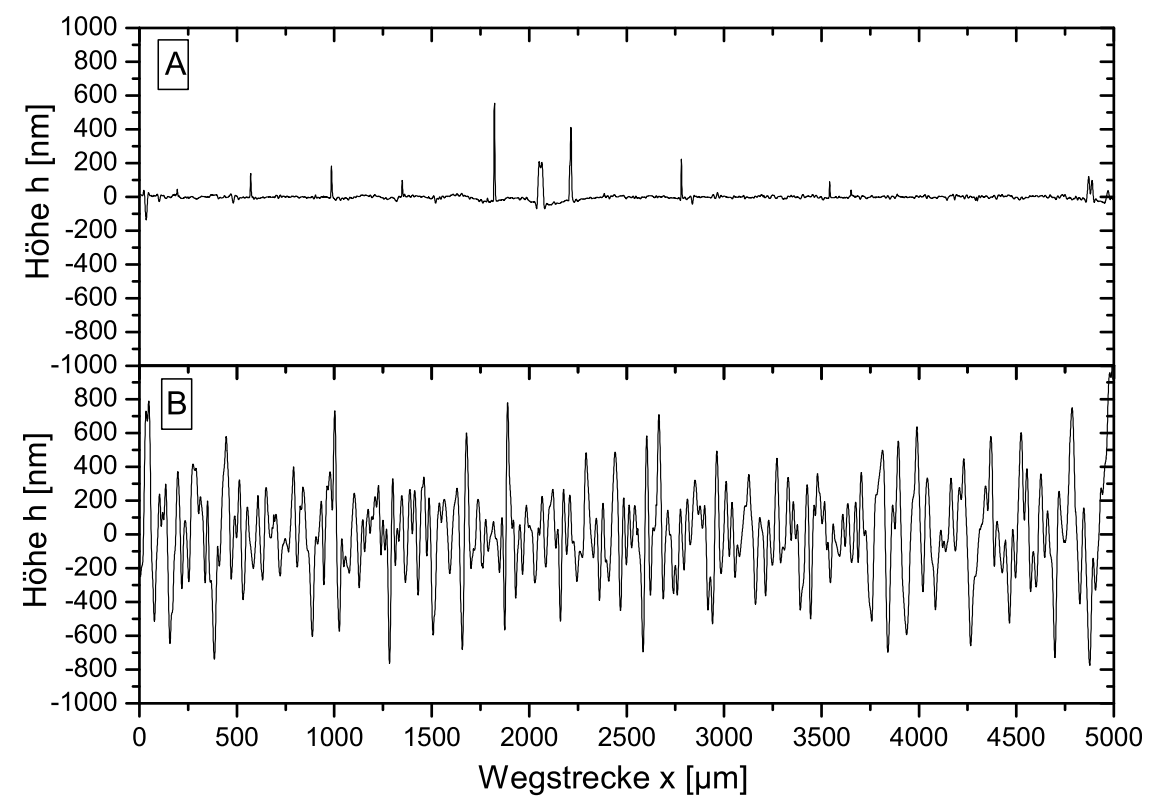

Abbildung 46: Zwei Beispiele für Fourier-gefilterte Höhenprofile der Probenoberfläche von laserhydrierten $c$-Si-Proben ( $p=3$ bar). (A) 64 Laserpulse der Fluenz F=3 J/ $\mathrm{cm}^{2}$, (B) 64 Laserpulse der Fluenz $F=5 \mathrm{~J} / \mathrm{cm}^{2}$ (oberhalb der Plasmaschwelle)

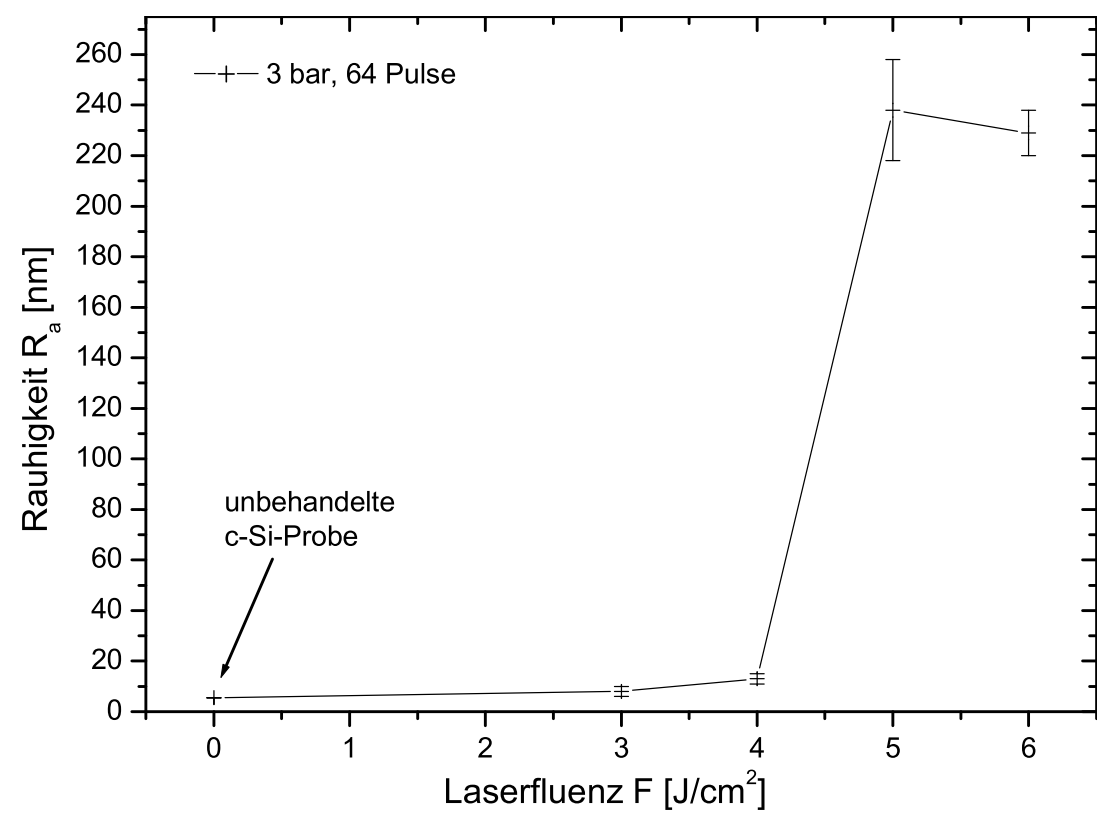

Abbildung 47: Oberflächenrauigkeit von laserhydrierten c-Si-Proben in Abhängigkeit von der Laserfluenz F (64 Laserpulse, $p=3$ bar). Nach Überschreiten der Plasmaschwelle bei $F_{\text {plas }} \approx 4 \mathrm{~J} / \mathrm{cm}^{2}$ nimmt die Oberflächenrauigkeit sprunghaft zu. 


\section{Schädigung der Gitterstruktur}

Die Schädigung der laserhydrierten c-Si-Proben wurde mittels RBS-Channeling-Messungen am IONAS bestimmt. Die normierte Schädigung $\chi_{\text {norm }}$ ist in Abb. 48 als Funktion der Tiefe dargestellt. Für die Schädigung der laserhydrierten Proben gilt ebenfalls, dass der geschädigte Bereich der Probe umso tiefer reicht, je höher die verwendete Laserfluenz war. Auffällig ist außerdem, dass unterhalb $\mathrm{F}=4 \mathrm{~J} / \mathrm{cm}^{2}$, d.h. ohne Zünden des Plasmas, in Si keine Schädigung auftritt. Aufschmelzen und Erstarren der Matrix führen in diesem Fall zur epitaktischen Rekristallisation.

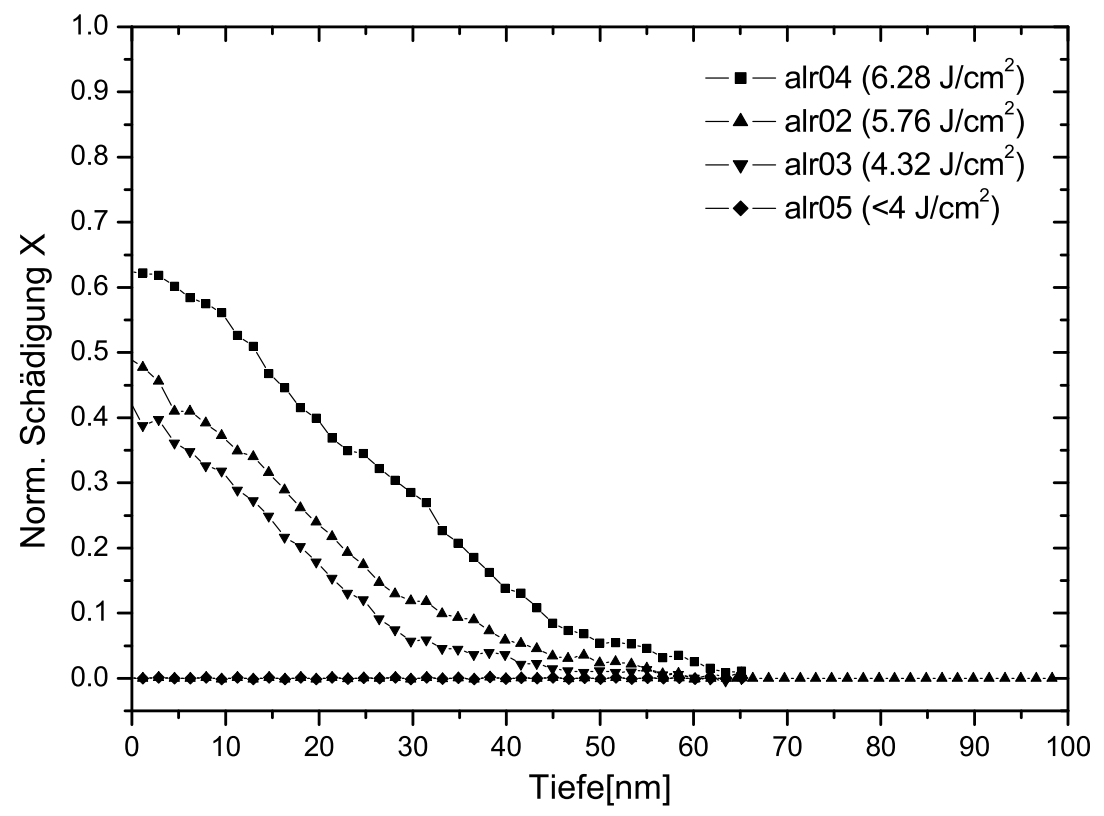

Abbildung 48: Schadensprofile von c-Si-Proben, die bei den angegebenen Laserfluenzen F (64 Laserpulse, $p=1$ bar) laserhydriert wurden

\section{Zusammenfassung}

Die Ergebnisse aus den Untersuchungen zur Wasserstoffaufnahme und Schädigung sind noch einmal in Tabelle 10 zusammengefasst. Die Tabelle zeigt den integralen Wasserstoffgehalt $\mathrm{C}_{H}$ der Proben nach dem Laserhydrieren sowie die integrale Schädigung $\mathrm{X}_{\text {tot }}$ aus den Channeling-Messungen.

In der letzten Spalte der Tabelle wurde der Wasserstoffgehalt der geschädigten Proben auf das Integral der Schädigung bezogen. Hierzu wurde zunächst der Wasserstoffgehalt der nach dem Laserhydrieren ungeschädigten Probe ALR05 abgezogen:

$$
C_{H}^{c o r r}=C_{H}-C_{H}(A L R 05)
$$

Somit stellt $\mathrm{C}_{H}^{c o r r} / \mathrm{X}_{\text {tot }}$ ein Maß für den in den geschädigten Bereichen gebundenen Wasserstoff dar. Dabei wird vorausgesetzt, dass sich der Wasserstoff im wesentlichen an den Defekten anlagert. Tabelle 10 verdeutlicht, dass mit zunehmender Laserfluenz F sowohl 


\begin{tabular}{c|c|c|c|c} 
Probe & $\begin{array}{c}\text { Laserfluenz } \\
\mathrm{F}\left[\mathrm{J} / \mathrm{cm}^{2}\right]\end{array}$ & $\begin{array}{c}\text { integr. Schädigung } \\
\mathrm{X}_{\text {tot }}\left[1 \mathrm{E} 15 \mathrm{at} / \mathrm{cm}^{2}\right]\end{array}$ & $\begin{array}{c}\text { Wasserstoffgehalt } \\
\mathrm{C}_{H}\left[1 \mathrm{E} 15 \mathrm{H} / \mathrm{cm}^{2}\right]\end{array}$ & $\mathrm{C}_{H}^{\text {corr }} / \mathrm{X}_{\text {tot }}$ \\
\hline \hline ALR05 & $<4$ & 0 & $2.0(1)$ & 0 \\
\hline ALR03 & 4.3 & $40(2)$ & $3.0(1)$ & $0.025(5)$ \\
\hline ALR02 & 5.8 & $53(2)$ & $5.6(3)$ & $0.068(7)$ \\
\hline ALR04 & 6.3 & $89(4)$ & $10.8(4)$ & $0.099(7)$ \\
\hline
\end{tabular}

Tabelle 10: Vergleich von Wasserstoffaufnahme $C_{H}$ und Schädigung $X_{\text {tot }}$ für verschiedene Laserfluenzen $F . C_{H}^{\text {corr }}=C_{H}-C_{H}(A L R 05)$ bezeichnet den Wasserstoffgehalt der Probe abzüglich des Wertes $C_{H}($ ALR05 $)=1.99 \times 10^{15} \mathrm{H} / \mathrm{cm}^{2}$ der Probe ohne messbare Schädigung

die Schädigung $\mathrm{X}_{t o t}$, als auch der Wasserstoffgehalt $\mathrm{C}_{H}$ der Proben steigen. Wie in Abb.49 $\mathrm{zu}$ sehen ist, nimmt $\mathrm{C}_{H}^{c o r r} / \mathrm{X}_{\text {tot }}$ mit der Laserfluenz $\mathrm{zu}$, so dass offensichtlich der Anteil des Wasserstoffs in den geschädigten Bereichen der Probe mit steigender Laserfluenz zunimmt. Unter der Annahme, dass der Wasserstoff die geschädigten Bereiche der Probe

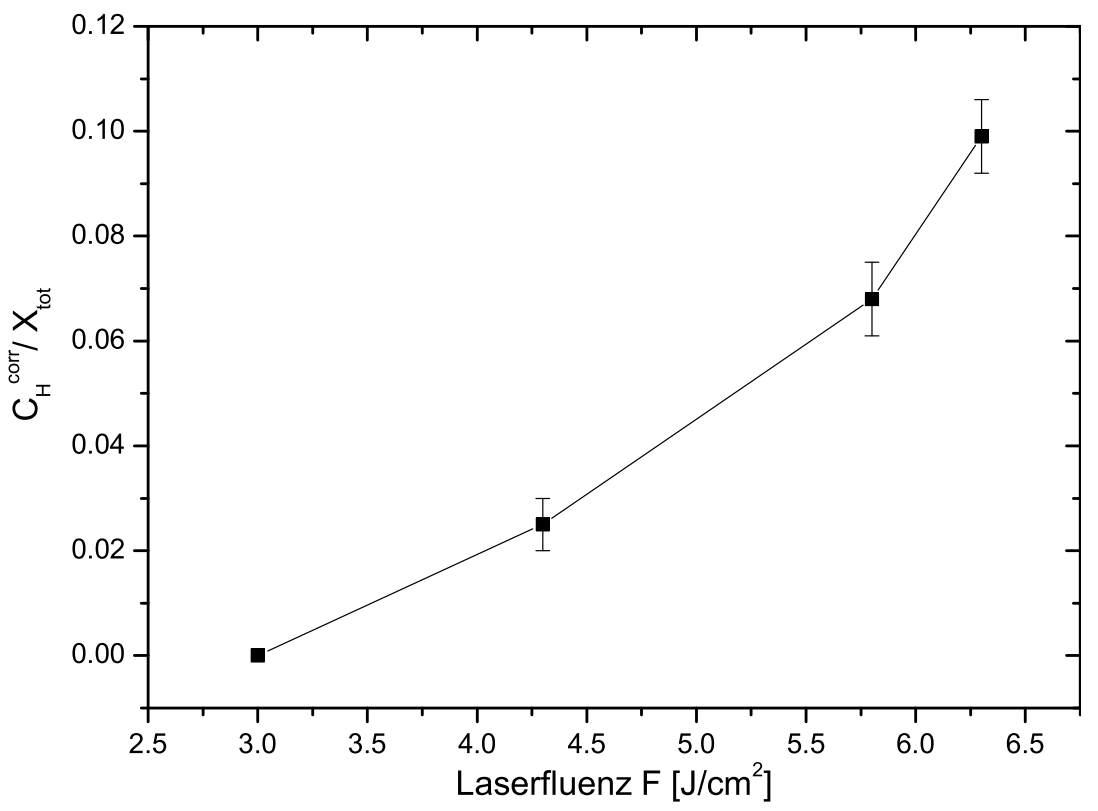

Abbildung 49: Auf die integrale Schädigung normierte Wasserstoffaufnahme $C_{H}^{\text {corr }} / X_{\text {tot }}$ als Funktion der Laserfluenz F

dekoriert und dass zusätzlich durch die Aufrauung der Oberfläche eine größere Menge Wasserstoff gebunden werden kann, lässt sich die Form der Tiefenprofile in Abb. 45 qualitativ verstehen. 


\subsection{Laserhydrieren von amorphisiertem Silizium}

Es ist bekannt, dass amorphes Silizium (a-Si) zum Teil völlig andere Materialeigenschaften aufweist als kristallines Silizium. Beispielsweise kann die elektrische Leitfähigkeit von a-Si bei Raumtemperatur durch Dotieren über mehr als zehn Größenordnungen verändert werden. Die Tatsache, dass es überhaupt möglich ist, amorphes Silizium zu dotieren, wurde erst 1975 von W. E. Spear und P. G. LeComber gezeigt [112].

Im nun folgenden Abschnitt soll das Verhalten von a-Si beim Laserhydrieren beschrieben werden. Hierzu wurden in einer ersten Serie von Experimenten durch die Bestrahlung von kristallinem Silizium mit $450 \mathrm{keV}$ Xe-Ionen ca. $400 \mathrm{~nm}$ dicke Schichten a-Si auf einem kristallinen Si-Substrat hergestellt (vgl. Abs. 5.2). Das dabei entstehende XeKonzentrationsprofil konnte zusätzlich als Markerschicht beim anschließenden Laserhydrieren verwendet werden. In einer zweiten Experiment-Reihe wurde kristallines Silizium mit Wasserstoffionen bei hohen Fluenzen (bis zu $10^{17} \mathrm{H} / \mathrm{cm}^{2}$ ) implantiert und damit eine vergrabene amorphe Schicht erzeugt. Bei diesen Proben wurde vor und nach dem Laserhydrieren die Wasserstofftiefenverteilung mittels NRA bestimmt, um auch Aussagen über die Wasserstoffdiffusion während des Laserhydrierens zu erhalten.

\subsubsection{Laserhydrieren von Xe-implantiertem Silizium}

Bei den in diesem Abschnitt untersuchten Proben handelt es sich um Doppelschichtsysteme, bestehend aus einer ca. $400 \mathrm{~nm}$ dicken Deckschicht aus a-Si auf kristallinem Si-Wafermaterial. Zunächst werden RBS-Channeling-Messungen gezeigt, die dazu dienen, den Grad der Schädigung des Kristallgitters zu bestimmen und die kritische Fluenz $\phi_{k r i t}$ zu ermitteln, oberhalb derer sich eine vollständig amorphisierte Schicht ausgebildet hat.

\section{Amorphisierung durch Xe-Implantation}

Wie in Kapitel 4.1 bereits dargestellt, bilden sich bei der Bestrahlung mittels Xe-Ionen aufgrund der im Verhältnis zur Si-Matrix großen Ionenmasse Stoßkaskaden aus, die schon bei relativ niedrigen Fluenzen (ca. $10^{14} \mathrm{Xe} / \mathrm{cm}^{2}$ ) überlappen. In der Tiefe des Maximums der deponierten Energiedichte entsteht eine geschädigte Schicht, die mit fortschreitender Ionenbestrahlung zunächst zur Probenoberfläche und dann zu größeren Tiefen wächst. In Abbildung 50 sind Schadensprofile von Xe-implantierten Siliziumproben gezeigt, die mit RBS-Channeling ermittelt wurden. Bei niedriger Fluenz der Xe-Ionen $\left(\phi \leq 10^{13} \mathrm{Xe} / \mathrm{cm}^{2}\right)$ wird die kristalline Si-Matrix durch die Bestrahlung lediglich geringfügig geschädigt. Die normierte Schädigung $\chi_{\text {norm }}$ erreicht in einer Tiefe von $150 \mathrm{~nm}$ den maximalen Wert von $\chi_{\text {norm }}=0.1$. Bei einer Fluenz von $\phi(\mathrm{Xe})=10^{14} \mathrm{Xe} / \mathrm{cm}^{2}$ hat sich bereits eine amorphe Schicht bis zur Probenoberfläche hin ausgebildet. Eine noch größere Xe-Fluenz $\left(10^{16} \mathrm{Xe} / \mathrm{cm}^{2}\right)$ führt zu einem Wachstum des amorphisierten Bereichs in das Probenvolumen hinein. Da für die spätere Nutzung des Xenon als Markersignal ein etwas höherer Xe-Gehalt nützlich ist, wurde für die meisten Proben eine Fluenz von $\phi(\mathrm{Xe})=5 \times 10^{15} \mathrm{Xe} / \mathrm{cm}^{2}$ gewählt, was einer maximalen Xe-Konzentration von ca. 0.8 at\% entspricht. 


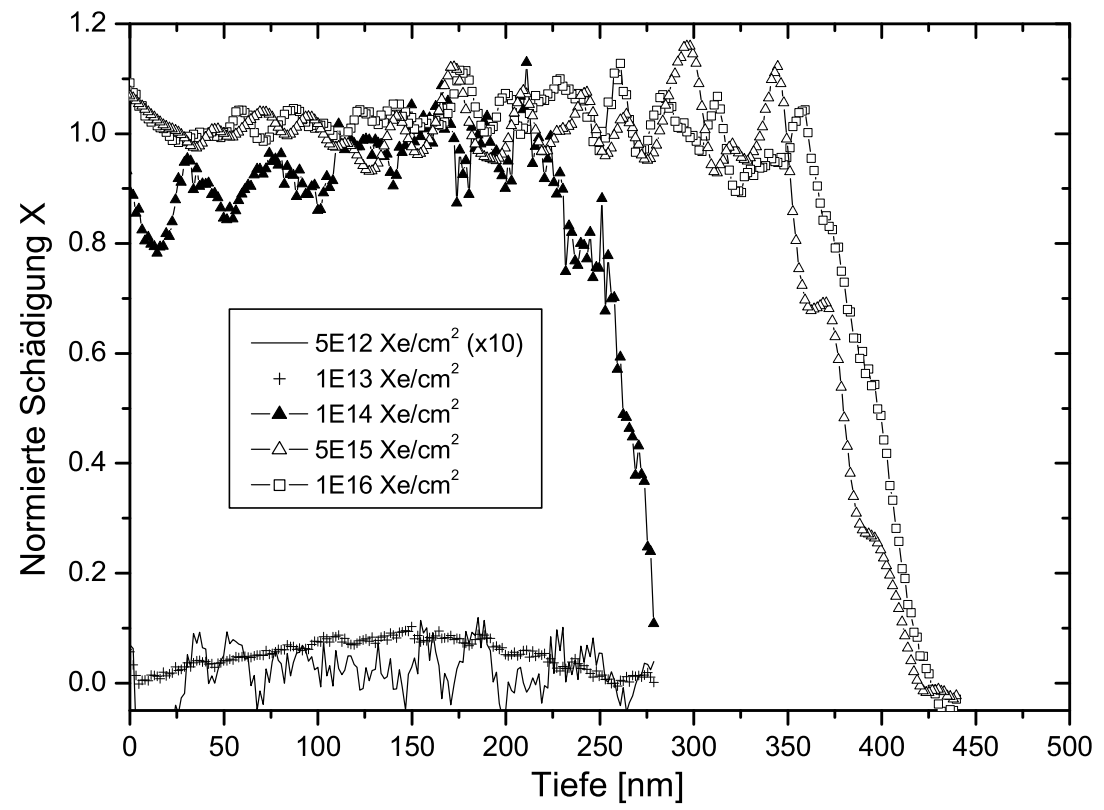

Abbildung 50: Schadensprofile von c-Si-Proben, die mit den angegebenen Xe-Fluenzen implantiert wurden. Die Energie der Xe-Ionen betrug E=450 keV. Das Schadensprofil der mit $\phi=5 \times 10^{12} \mathrm{Xe} / \mathrm{cm}^{2}$ implantierten Probe ist zehnfach vergrößert dargestellt.

\section{Oberflächenrauigkeit nach dem Laserhydrieren}

Abbildung 51 zeigt die Oberflächenrauigkeit $\mathrm{R}_{a}$ von laserhydrierten a-Si-Proben in Abhängigkeit von der Laserfluenz F. Die hier dargestellten Proben wurden mit 16 Laserpulsen in 2 bar Wasserstoff belichtet, ohne mäanderförmige Bestrahlung. Um auch höhere Fluenzen bis zu $5 \mathrm{~J} / \mathrm{cm}^{2}$ erreichen zu können, wurde wie schon bei der Bestrahlung der kristallinen Siliziumproben der Laser-Rohstrahl verwendet. Die Verwendung eines Homogenisators hätte einen großen Verlust der verfügbaren Laserintensität zur Folge gehabt.

Zunächst fällt auf, dass sich der Wert der Oberflächenrauigkeit $\mathrm{R}_{a}$ für geringe Laserfluenzen $\mathrm{F} \leq 2 \mathrm{~J} / \mathrm{cm}^{2}$ nicht wesentlich ändert. Er liegt mit $\mathrm{R}_{a}$ (Laser) $\approx 4.5(5) \mathrm{nm}$ nicht höher als der Wert $\mathrm{R}_{a}$ (Xe-impl.) $\approx 4.6(2) \mathrm{nm}$ für Si-Proben, die ausschließlich mit Xe-Ionen implantiert wurden. Ab einer Laserfluenz von $3 \mathrm{~J} / \mathrm{cm}^{2}$ steigt der Wert der Oberflächenrauigkeit an und erreicht bei $\mathrm{F}=5 \mathrm{~J} / \mathrm{cm}^{2}$ den sechzigfachen Wert der lediglich Xe-implantierten aber nicht laserbehandelten Proben.

Mit dem Rauigkeitswert $\mathrm{R}_{a}$ nimmt auch dessen Fehler $\Delta \mathrm{R}_{a}$ stark zu. Da es sich bei $\Delta \mathrm{R}_{a}$ hauptsächlich um statistische Fehler handelt, die durch das Vermessen unterschiedlicher Bereiche auf der Probenoberfläche zustande kommen, ist $\Delta \mathrm{R}_{a}$ ein Maß für die Inhomogenität der laserbehandelten Probenoberfläche. Während das Laserprofil auf den Proben nach der Bestrahlung mit Laserfluenzen $\mathrm{F} \leq 3 \mathrm{~J} / \mathrm{cm}^{2}$ ohne Vergrößerung kaum zu erkennen ist, weisen die bei höheren Laserfluenzen bestrahlten Proben deutliche Unebenheiten auf.

\section{Schädigung und Rekristallisation}

Wie in Abschnitt 4.3 bereits erläutert, führt der laserinduzierte Temperaturanstieg der Probenoberfläche zu einer erhöhten Mobilität der Atome, wodurch es zu Umordnungs- 


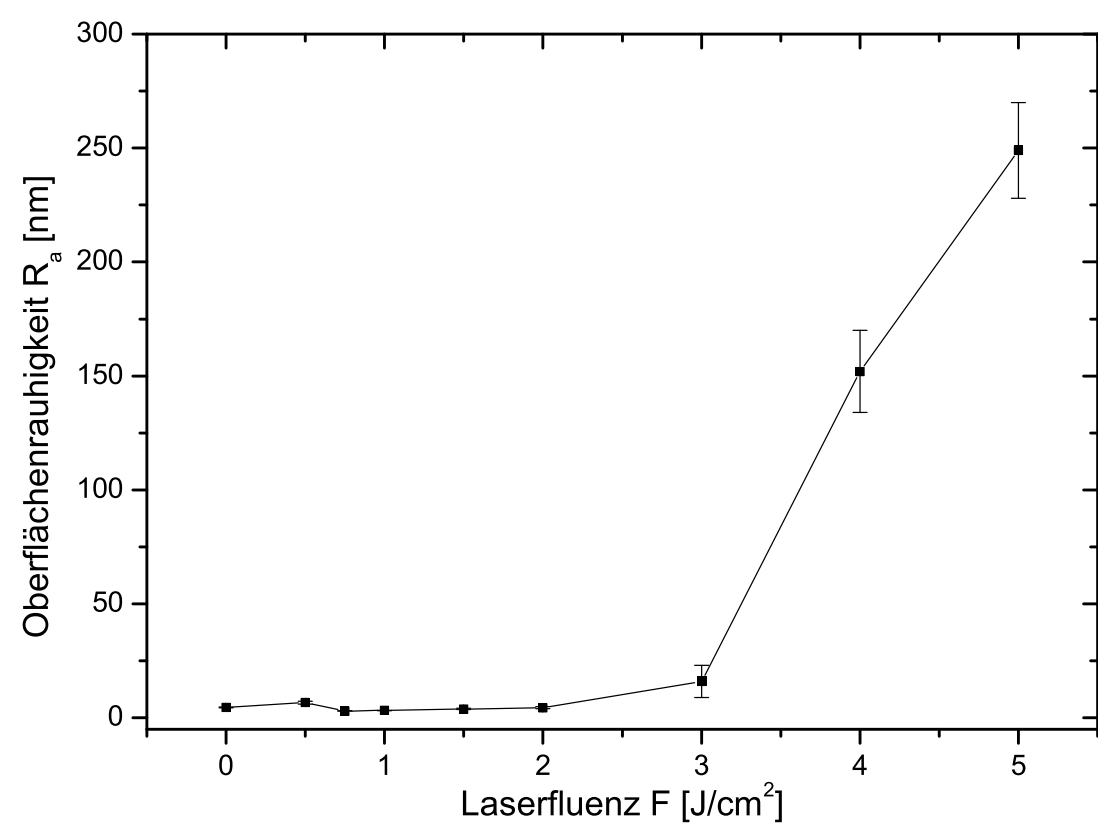

Abbildung 51: Oberflächenrauigkeit von Xe-amorphisierten Si-Proben die mit 16 Laserpulsen bei 2 bar laserhydriert wurden.

prozessen und Rekristallisation des amorphen Siliziums kommt. Um den Einfluss der Rekristallisation auf die Tiefenverteilungen sowohl des implantierten Xenons, als auch des durch das Laserhydrieren aufgenommenen Wasserstoffs zu ermitteln, wurden vor und nach dem Laserhydrieren RBS-Schadensprofile gemessen.

Abbildung 52 zeigt im oberen Teil die Tiefenverteilung der normierten Schädigung $\chi_{n o r m}(z)$ für eine Xe-amorphisierte Si-Probe $\left(\phi(\mathrm{Xe})=5 \times 10^{15} \mathrm{Xe} / \mathrm{cm}^{2}\right)$ vor und nach dem Laserhydrieren mit 16 Laserpulsen der relativ geringen Laserfluenz $\mathrm{F}=0.5 \mathrm{~J} / \mathrm{cm}^{2}$. Deutlich ist eine Abnahme der Schädigung ab einer Tiefe von ca. $100 \mathrm{~nm}$ zu sehen. Allerdings findet auch an der Grenzfläche amorph/kristallin $\left(\mathrm{z}_{a / c} \approx 390 \mathrm{~nm}\right)$ keine vollständige Rekristallisation statt, sondern die normierte Schädigung hat hier den Wert $\chi_{\text {norm }}\left(z_{a / c}\right)=0.5$. Die Schädigung steigt in Richtung der Probenoberfläche linear an.

Das Xe-Tiefenprofil, das im unteren Teil von Abb.52 abgebildet ist, zeigt dagegen keinerlei Veränderung durch die Laserbestrahlung. Lage und Form des Implantationsprofils stimmen vor und nach dem Laserhydrieren überein. Gemäß den Überlegungen aus Abs. 4.3 muss davon ausgegangen werden, dass es durch die Laserbestrahlung zu einer teilweisen Rekristallisation ausgehend von der Grenzfläche zwischen dem amorphisierten Bereich und dem kristallinen Substrat kam. In der zuvor amorphisierten Schicht überwiegen jedoch die Defektstrukturen, so dass die normierte Schädigung $\chi_{\text {norm }}$ den Wert 0.5 nirgends unterschreitet. Außerdem muss ebenfalls von einem Dechanneling aufgrund des noch in der Schicht verbliebenen Xenons ausgegangen werden. Dieser Dechanneling-Effekt des Xenons ist mittels RBS-Channeling nicht von Defekten zu unterscheiden.

Wird die Laserfluenz erhöht, so ergibt sich ein völlig anderes Bild: Die in Abb. 53 dargestellte Probe wurde bei sonst gleichen Parametern mit der Laserfluenz $\mathrm{F}=1 \mathrm{~J} / \mathrm{cm}^{2}$ bestrahlt. Im oberen Teil ist wieder das Schadensprofil dargestellt, das nach dem Laserhydrieren über die gesamte Schichtdicke gemittelt eine Schädigung von $\chi_{\text {norm }} \approx 0.25$ besitzt und an keiner Stelle den Wert $\chi_{\text {norm }}=1$ erreicht. Es liegen also keine vollständig 


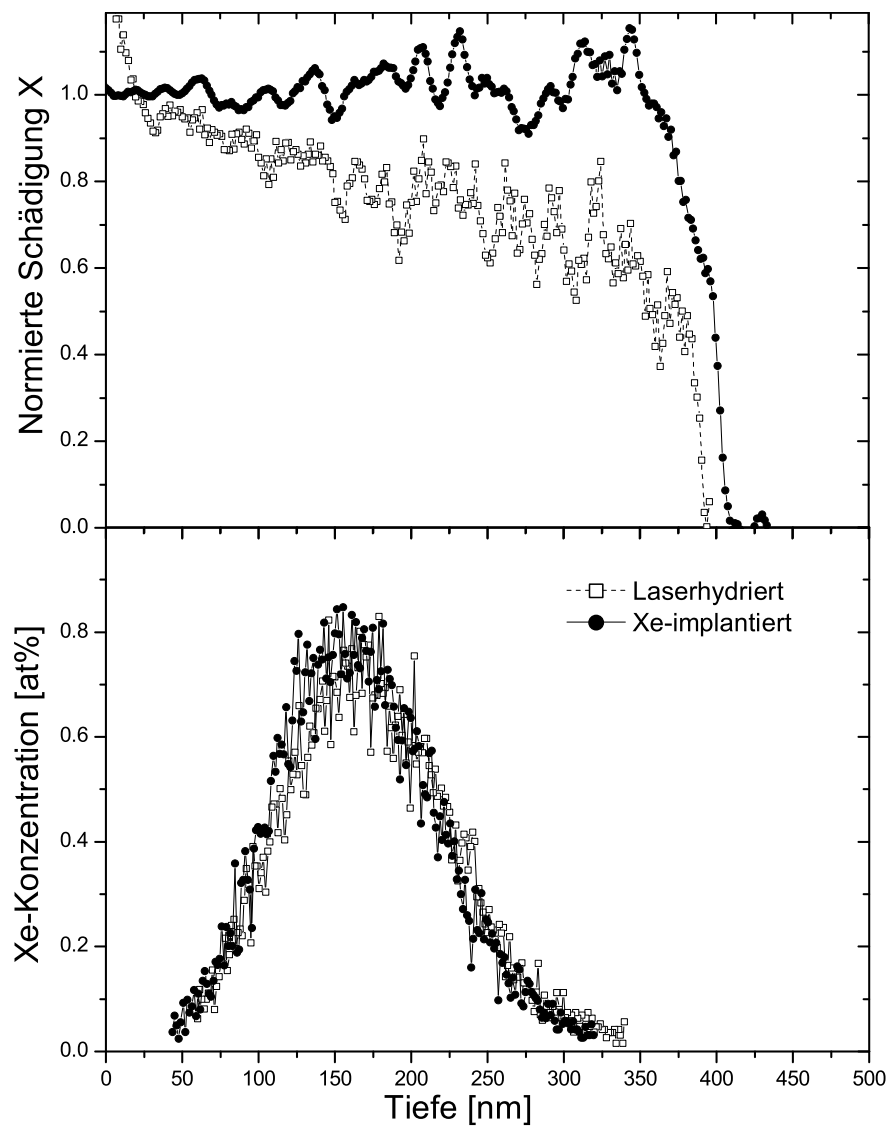

Abbildung 52: Tiefenverteilung der normierten Schädigung $\chi(x)$ (oben) und Xe-Tiefenprofil (unten) einer Xe-amorphisierten Si-Probe, vor dem Laserhydrieren (offene Quadrate), und nach dem Laserhydrieren (gefüllte Kreise). Die Probe wurde mit 16 Laserpulsen der Fluenz F=0.5 J/cm ${ }^{2}$ bei einem Wasserstoffdruck von 2 bar bestrahlt.

amorphen Bereiche mehr vor. Wichtig ist an dieser Stelle, dass sich die Dicke der geschädigten Schicht durch das Laserhydrieren nicht geändert hat. Lediglich der Mittelwert der Schädigung hat sich gegenüber dem Schadensprofil bei $F=0.5 \mathrm{~J} / \mathrm{cm}^{2}$ (Abb. 52 halbiert. Das im unteren Teil der Abbildung dargestellte Xe-Tiefenprofil zeigt ebenfalls einige Veränderungen durch das Laserhydrieren. Zum einen ist die Maximalkonzentration von 0.65 at\% auf 0.5 at\% abgefallen. Zum anderen zeigt das Xe-Profil nach dem Laserhydrieren einen Ausläufer, der bis an die Probenoberfläche reicht. Der integrale Xe-Gehalt hat bei dieser Probe nur wenig abgenommen $\left(\mathrm{C}_{\text {nach }}(\mathrm{Xe}) / \mathrm{C}_{\text {vor }}(\mathrm{Xe})=0.83(3)\right)$.

Wird die Laserfluenz weiter auf $\mathrm{F}=1.5 \mathrm{~J} / \mathrm{cm}^{2}$ erhöht, dann verschwindet die Schädigung nach der Bestrahlung komplett, wie im oberen Teil von Abbildung 54 zu sehen ist. Folglich reicht in diesem Fall die Energie der Laserstrahlung für eine Neuorientierung der Si-Atome aus und es liegt eine vollständige epitaktische Rekristallisation der vorher amorphen Deckschicht vor. Das im unteren Teil von Abb. 54 dargestellte Xe-Tiefenprofil zeigt, dass mit der epitaktischen Rekristallisation des Siliziums beinahe alle Xe-Atome aus der laserhydrierten Probe herausdiffundiert sind. Im Hinblick auf die Xe-Verteilung nach der Bestrahlung mit $\mathrm{F}=1 \mathrm{~J} / \mathrm{cm}^{2}$ (Abb. 53) muss davon ausgegangen werden, dass die Diffusion des Xenons zur Probenoberfläche hin stattfindet. Diese Vermutung wird auch durch die Form des noch vorhandenen Xe-Tiefenprofils in Abb. 54 bestätigt. Das flache Xenon- 


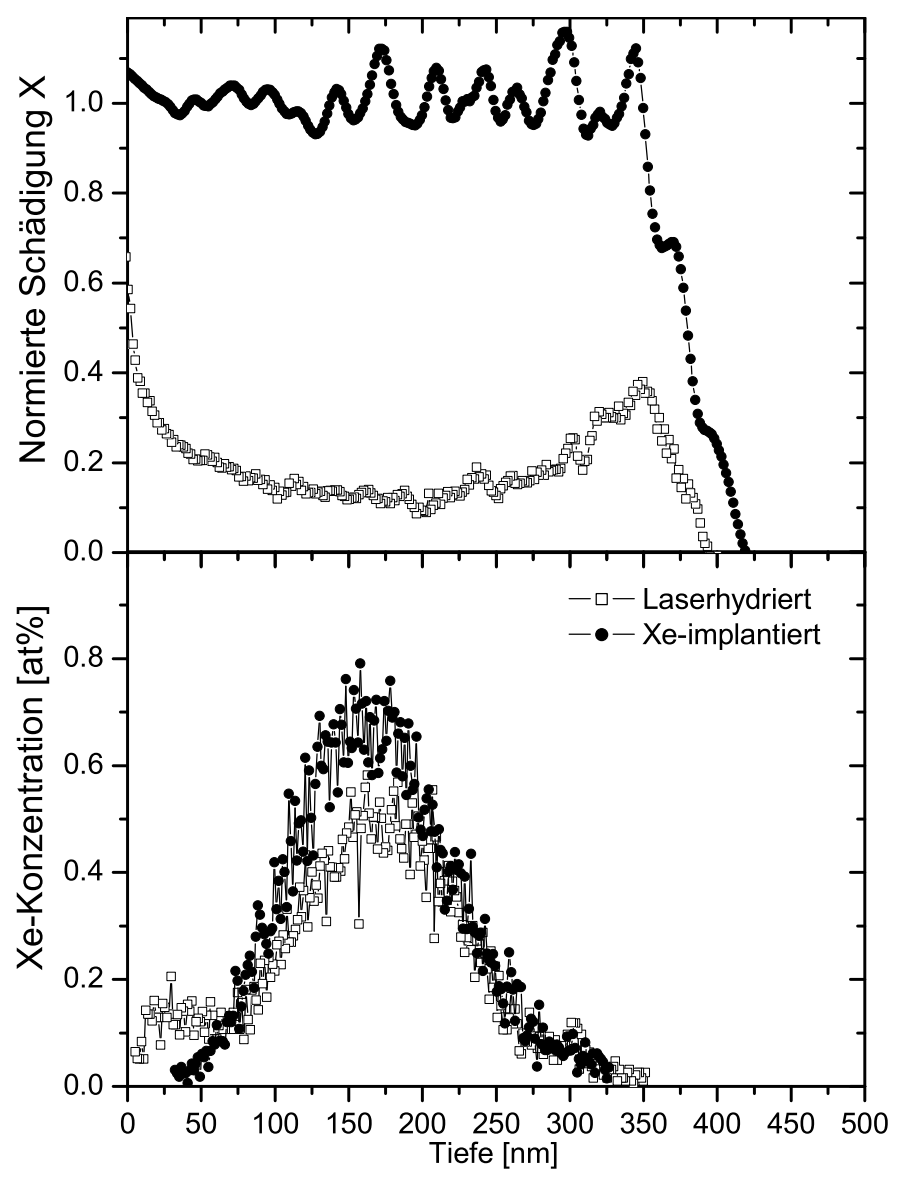

Abbildung 53: Tiefenverteilung der normierten Schädigung $\chi(x)_{n o r m}$ (oben) und Xe-Tiefenprofil (unten) einer Xe-amorphisierten Si-Probe, vor dem Laserhydrieren (offene Quadrate), und nach dem Laserhydrieren (gefüllte Kreise). Die Probe wurde mit 16 Laserpulsen der Fluenz $F=0.96 \mathrm{~J} / \mathrm{cm}^{2}$ bei einem Wasserstoffdruck von 2 bar bestrahlt.

profil nach dem Laserhydrieren reicht von der Probenoberfläche bis in eine Tiefe von ca. 300 nm. Dieser Wert entspricht der Tiefe der rechten Flanke des Implantationsprofils. Diese Beobachtung macht deutlich, dass es sich tatsächlich um epitaktische Rekristallisation der amorphen Schicht und nicht um einen Ablationsprozess handelt. Würde nämlich die amorphe Deckschicht durch die Laserbestrahlung einfach abgetragen, so würden die RBS-Channeling-Messungen zwar ebenfalls ein ungestörtes Profil ergeben. Allerdings sollte dann mit der amorphen Deckschicht auch das implantierte Xenon abgetragen werden. Dies ist jedoch eindeutig nicht der Fall, wie das Profil des übriggebliebenen Xenons in Abb. 54 (unten) beweist.

Alle bisher in diesem Abschnitt aufgeführten Ergebnisse bezogen sich auf Xe-amorphisierte Siliziumproben, die jeweils mit 16 Laserpulsen mit verschiedenen Laserfluenzen bestrahlt worden waren. Es ergibt sich die Frage, welche Veränderungen ein einzelner Laserpuls hervorruft.

Abbildung 55 zeigt das Schädigungsprofil und die Xe-Tiefenverteilung für eine Xe-amorphisierte Si-Probe vor und nach der Bestrahlung mit 1 Laserpuls der Fluenz $\mathrm{F}=5 \mathrm{~J} / \mathrm{cm}^{2}$ bei einem Wasserstoffdruck von 2 bar. Anhand des Schädigungsprofils im oberen Teil der Abbildung ist ersichtlich, dass bereits 1 Laserpuls der relativ hohen Fluenz $\mathrm{F}=5 \mathrm{~J} / \mathrm{cm}^{2}$ aus- 


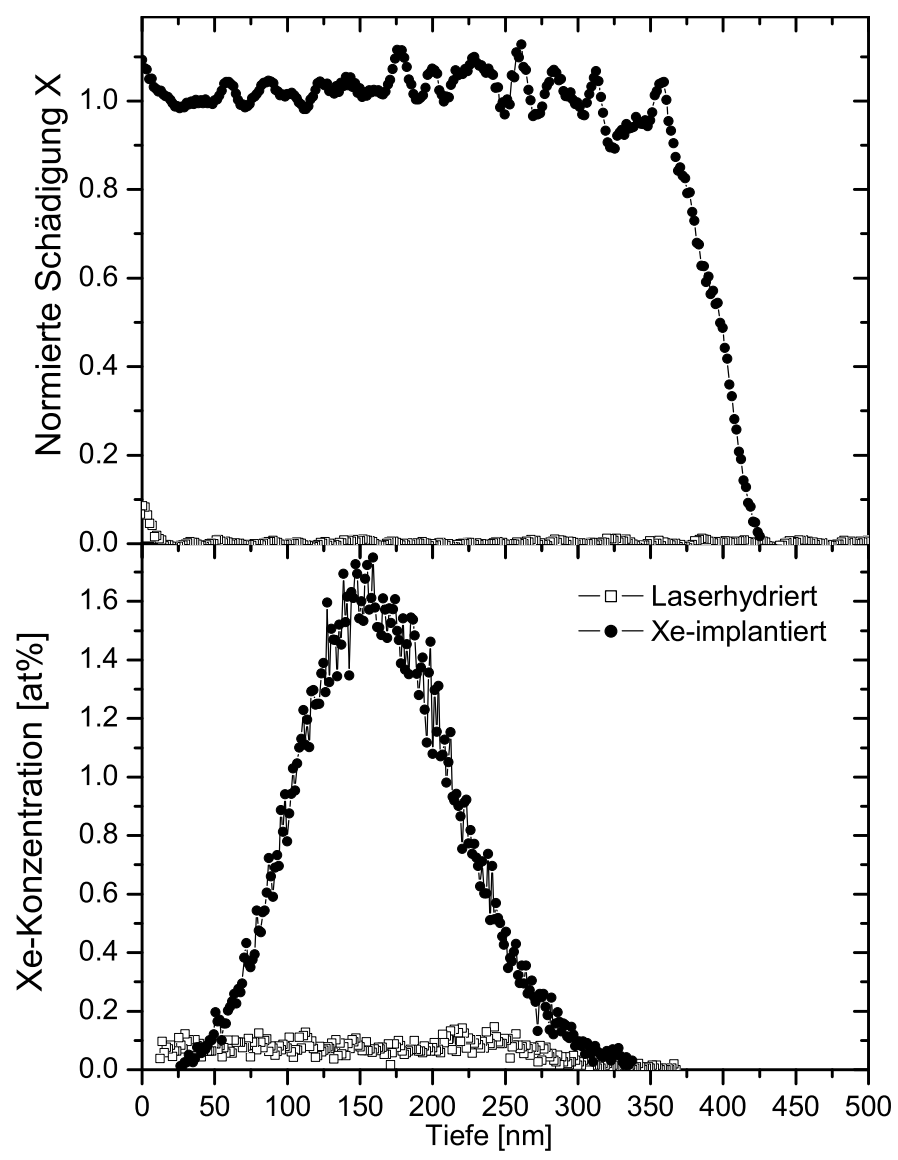

Abbildung 54: Tiefenverteilung der normierten Schädigung $\chi(x)_{n o r m}$ (oben) und Xe-Tiefenprofil (unten) einer Xe-amorphisierten Si-Probe, vor dem Laserhydrieren (hohle Quadrate), und nach dem Laserhydrieren (gefüllte Kreise). Die Probe wurde mit 16 Laserpulsen der Fluenz $F=1.5 \mathrm{~J} / \mathrm{cm}^{2}$ bei einem Wasserstoffdruck von 2 bar bestrahlt.

reicht, um die $400 \mathrm{~nm}$-dicke amorphe Schicht epitaktisch zu rekristallisieren. Die normierte Schädigung fällt von $\chi_{\text {norm }}=0.4$ an der Probenoberfläche innerhalb der oberen $30 \mathrm{~nm}$ auf $\chi_{n o r m} \approx 0$. Dieses Ergebnis entspricht dem im vorigen Abschnitt beim Laserhydrieren von kristallinem Silizium beschriebenen Verhalten. Dort resultierte aus der Bestrahlung mit $\mathrm{F}>4 \mathrm{~J} / \mathrm{cm}^{2}$ ebenfalls ein geschädigter Bereich unterhalb der Oberfläche. Auch bei der in Abb. 55 gezeigten Probe zündete bei der Laserbestrahlung ein Plasma, was sich auch an der großen Oberflächenrauigkeit $\mathrm{R}_{a}=249(21) \mathrm{nm}$ bemerkbar macht (vgl. Abb. 51). Das im unteren Teil der Abbildung dargestellte Xe-Tiefenprofil zeigt ebenfalls eine starke Veränderung durch die Laserbestrahlung. Das Maximum der Xe-Verteilung hat sich von $160 \mathrm{~nm}$ zu 50 nm in Richtung der Probenoberfläche verschoben. Das Xe-Profil fällt annähernd linear ab bis in eine Tiefe von $400 \mathrm{~nm}$. Dies entspricht der Dicke des zuvor amorphisierten Bereichs der Probe. Die Verteilung der Xenonatome hat sich durch die Laserbestrahlung beinahe über den gesamten amorphisierten Bereich ausgedehnt. Nahe der Oberfläche bis in eine Tiefe von ca. $15 \mathrm{~nm}$ ist kein Xenon vorhanden. Da das Schädigungsprofil in diesem Bereich eine relativ starke Schädigung zeigt, kann davon ausgegangen werden, dass die Xe-Atome in diesem Bereich zur Probenoberfläche ausgasen. Der integrale Xe-Gehalt hat durch die Laserbestrahlung stark abgenommen $\left(\mathrm{C}_{\text {nach }}(\mathrm{Xe}) / \mathrm{C}_{\text {vor }}(\mathrm{Xe})=0.45(2)\right)$. 


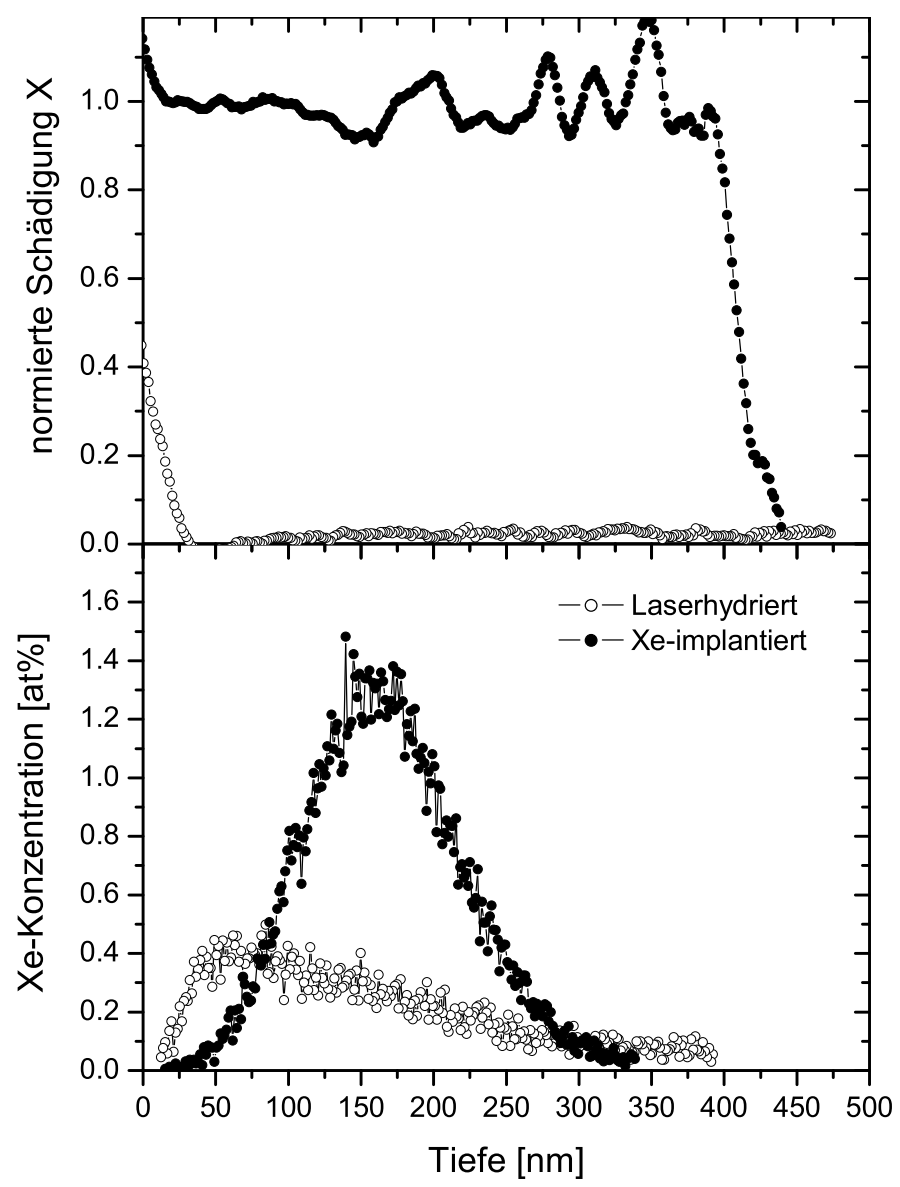

Abbildung 55: Tiefenverteilung der normierten Schädigung $\chi(x)_{n o r m}$ (oben) und Xe-Tiefenprofil (unten) einer Xe-amorphisierten Si-Probe, vor dem Laserhydrieren (offene Quadrate), und nach dem Laserhydrieren (gefüllte Kreise). Die Probe wurde mit 1 Laserpuls der Fluenz F=5 J/ $\mathrm{cm}^{2}$ bei einem Wasserstoffdruck von 2 bar bestrahlt.

Ein weiterer wichtiger Aspekt kann an Abb. 55 abgelesen werden. Die gezeigte XeKonzentration der Probe nach der Laserbestrahlung von maximal c $(\mathrm{Xe})=0.45 \mathrm{at} \%$ führt nur zu einem sehr geringen Dechanneling in der im oberen Teil abgebildeten Schadensverteilung von $\chi_{\text {norm }} \leq 0.04$. Das heißt, dass diese geringe Xe-Konzentration nur zu einer sehr geringen Störung der Si-Matrix führt. Im Umkehrschluss kann folglich der Beitrag der Xe-Atome zur gemessenen Schadensverteilung der Si-Proben vernachlässigt werden. Zur Vervollständigung der Untersuchungen zur Kristallinität nach dem Laserhydrieren wurden an einigen laserhydrierten Proben XRD-Messungen unter streifendem Einfall $\left(0.5^{\circ}\right)$ vorgenommen (Grazing Incidence X-Ray Diffraction, GI-XRD). In dieser Geometrie wird die Bragg-Bedingung nur dann erfüllt, wenn es sich bei der untersuchten Probe um polykristallines Material mit zufälliger Orientierung der Kristallite handelt. Wie Abb. 56 zeigt, sind im GI-XRD-Spektrum deutliche Reflexe zu sehen, die eindeutig mit den Kristallebenen von kristallinem Si identifiziert werden können. 


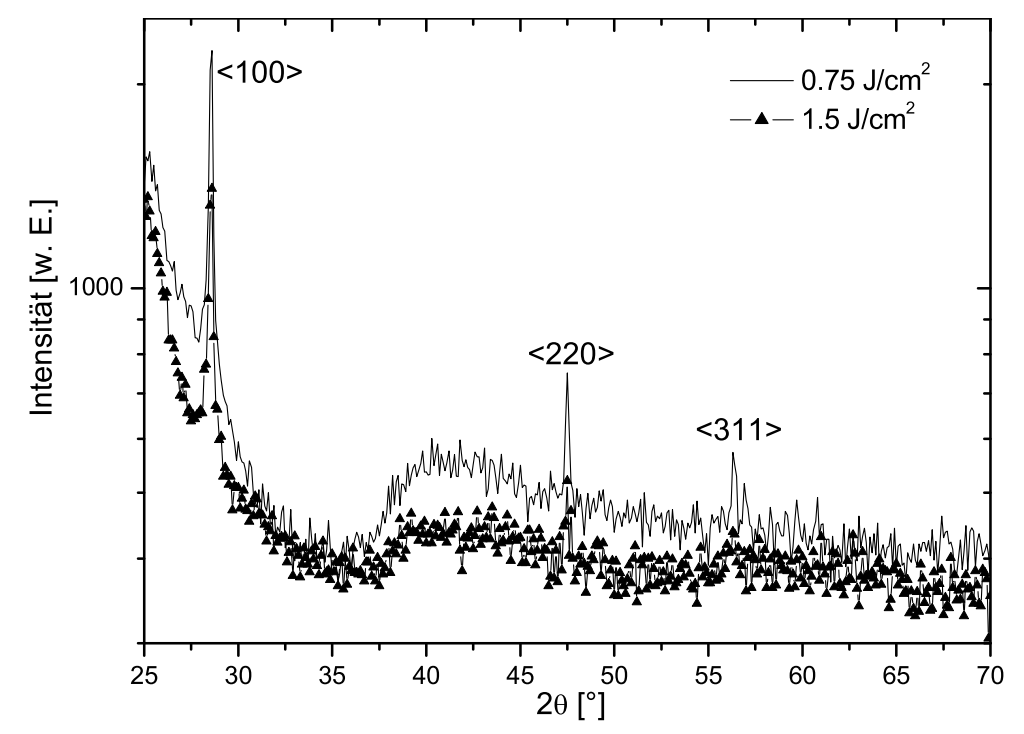

Abbildung 56: GI-XRD-Spektren von Xe-amorphisierten Si-Proben, die mit 16 Laserpulsen der Fluenz $0.75 \mathrm{~J} / \mathrm{cm}^{2}$ bzw. $1.5 \mathrm{~J} / \mathrm{cm}^{2}$ bei 2 bar Wasserstoffdruck laserhydriert wurden. Die deutlich sichtbaren Reflexe sind ein Indiz für Polykristallinität.

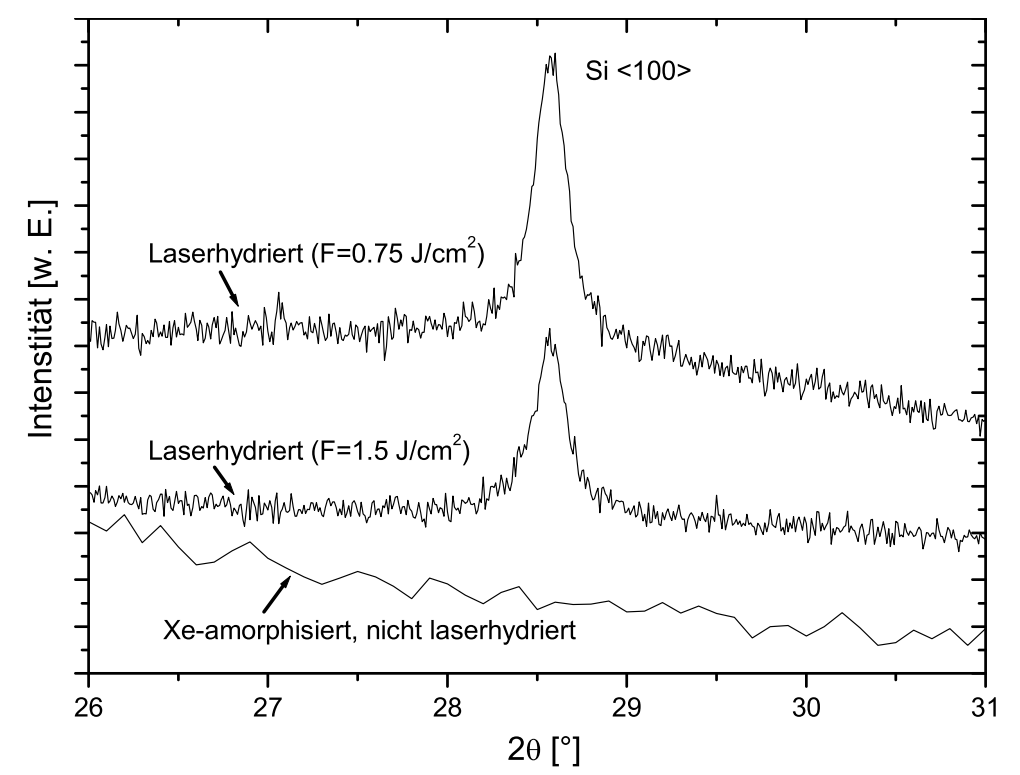

Abbildung 57: GI-XRD-Spektren von Xe-amorphisierten Si-Proben, die mit 16 Laserpulsen der Fluenz $0.75 \mathrm{~J} / \mathrm{cm}^{2}$ bzw. $1.5 \mathrm{~J} / \mathrm{cm}^{2}$ bei 2 bar Wasserstoffdruck laserhydriert wurden. 
Die Winkel der drei stärksten Röntgenreflexe $(<100>,<200>$ und $<311>)$ stimmen gut mit Literaturwerten überein [113]. Zusätzlich wurde bei den gezeigten Proben die mittlere Kristallitgröße $\bar{D}$ ebenfalls mittels XRD-Messungen bestimmt. Die Kristallitgröße lässt sich prinzipiell mit der Scherrer-Formel ermitteln: $\bar{D}=\frac{0.9 \lambda}{B \cos \theta_{B}}(\lambda$ : Röntgen-Wellenlänge, B: Halbwertsbreite des untersuchten Reflexes und $\theta_{B}$ : zugehöriger Bragg-Winkel). Die Ergebnisse in Tabelle 11 wurden durch eine Entfaltung des gemessenen XRD-Spektrums unter Berücksichtigung des Aufösungsvermögens der benutzten XRD-Apparatur erhalten. Der in der letzten Spalte angegebene Mittelwert für die Kristallitgröße beträgt bei beiden Proben übereinstimmend $\bar{D}=64(8) \mathrm{nm}$.

\begin{tabular}{c|c|c|c|c}
$\begin{array}{c}\text { Laserfluenz } \\
{\left[\mathrm{J} / \mathrm{cm}^{2}\right]}\end{array}$ & $\begin{array}{c}\bar{D}(<100>) \\
{[\mathrm{nm}]}\end{array}$ & $\begin{array}{c}\bar{D}(<220>) \\
{[\mathrm{nm}]}\end{array}$ & $\begin{array}{c}\bar{D}(<311>) \\
{[\mathrm{nm}]}\end{array}$ & $\begin{array}{c}\bar{D} \\
{[\mathrm{~nm}]}\end{array}$ \\
\hline \hline 0.75 & 67 & 75 & 49 & $64(8)$ \\
\hline 1.5 & 68 & 76 & 49 & $64(8)$ \\
\hline
\end{tabular}

Tabelle 11: Mittlere Kristallitgrößen für zwei laserhydrierte Xe-amorphisierte Si-Proben, aufgeschlüsselt nach den Kristallachsen

\section{Wasserstoffaufnahme}

Nachdem im vorigen Abschnitt die zum Teil starken Veränderungen der Struktur und des Xe-Gehalts der amorphen Siliziumproben durch das Laserhydrieren dargestellt wurden, soll nun gezeigt werden, in welchem Maße es zu einem Wasserstoffeintrag in die laserhydrierten Proben kommt.

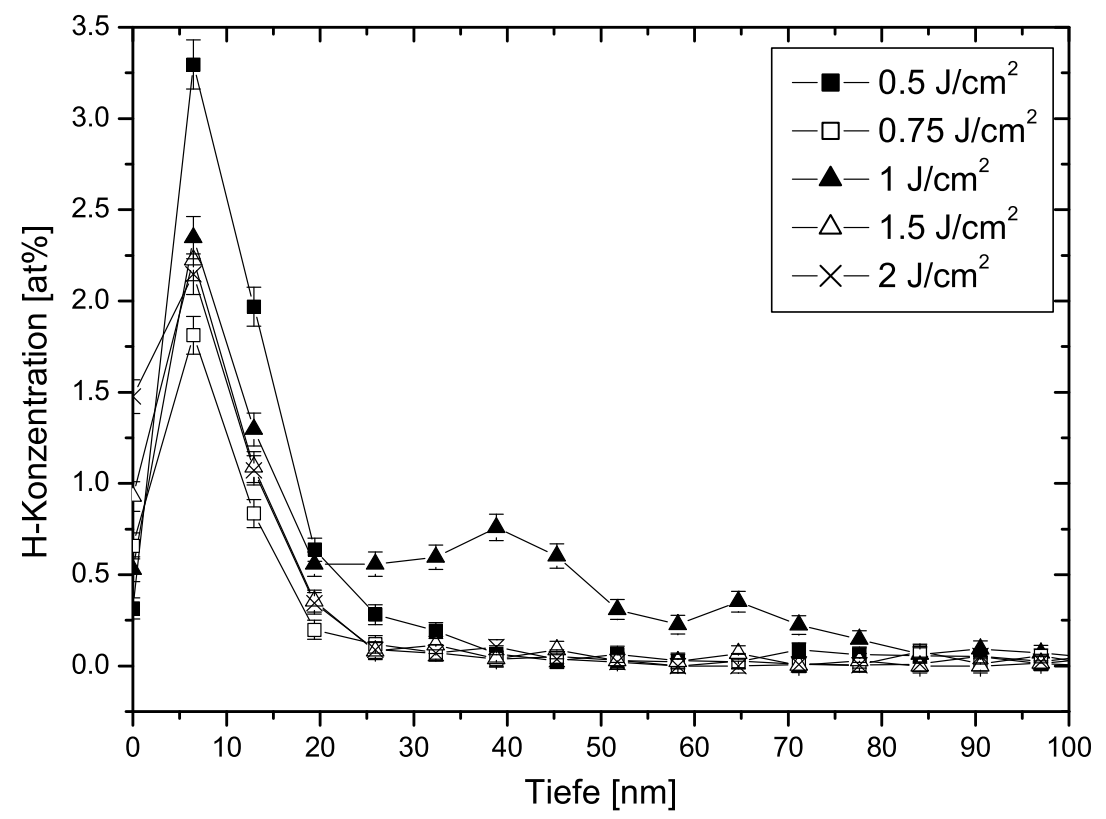

Abbildung 58: Wasserstofftiefenverteilung von Xe-amorphisierten Si-Proben, nach Laserhydrieren mit 16 Laserpulsen der angegebenen Fluenzen, jeweils bei einem Wasserstoffdruck von 2 bar. 
In Abbildung 58 sind Wasserstofftiefenprofile von Xe-amorphisierten Siliziumproben gezeigt, die mit 16 Laserpulsen bei einem Wasserstoffdruck von 2 bar laserhydriert wurden. Dabei werden zwei Aspekte deutlich. Zum einen erhöht sich mit zunehmender Laserfluenz die Wasserstoffkonzentration im oberflächennahen Bereich bis in eine Tiefe von ca. $10 \mathrm{~nm}$. Dieser Effekt wurde auch schon beim Laserhydrieren von c-Si gefunden (vgl. Abb. 45, S. 66) und konnte dort durch die Zunahme der Oberflächenrauigkeit (vgl. Abb. 47, S. 67) und dadurch erhöhte Wasserstoff-Adsorption an der Probenoberfläche erklärt werden.

Die hier vorliegenden a-Si-Proben zeigen jedoch eine Zunahme der Oberflächenrauigkeit erst bei wesentlich höheren Laserfluenzen (s. Abb. 51, S. 72). Für den in Abb. 58 dargestellten Bereich der Fluenz von $0.5 \leq \mathrm{F} \leq 3 \mathrm{~J} / \mathrm{cm}^{2}$ nimmt daher der Wasserstoffeintrag in den oberflächennahen Bereich tatsächlich mit zunehmender Laserfluenz zu.

Zweitens bildet sich mit zunehmender Laserfluenz ein Ausläufer des Wasserstoffprofils in das Probenvolumen hinein aus, der im Falle der bei $1 \mathrm{~J} / \mathrm{cm}^{2}$ laserhydrierten Probe bis in eine Tiefe von $80 \mathrm{~nm}$ reicht. Dieser Trend setzt sich jedoch bei höheren Fluenzen nicht fort.

Die in Abb. 59 gezeigten laserhydrierten Proben wurden bei sonst gleichen Parametern (16 Pulse, 2 bar) bei höherer Laserfluenz von 2-5 J/ $\mathrm{cm}^{2}$ bestrahlt. Zunächst fällt auf, dass die Erhöhung der Fluenz nicht zu einer Vergrößerung der Wasserstoffkonzentration an der Probenoberfläche führt. Allerdings ist auch die abfallende Flanke des Wasserstofftiefenprofils ins Probeninnere nur noch sehr schwach zu sehen. Die Spektren weisen nur noch an der Probenoberfläche einen erhöhten Wasserstoffgehalt auf, der mit dem stets vorhandenen Oberflächenpeak vergleichbar ist. In die Abb. 59 wurde auch das Wasserstoffprofil einer Probe aufgenommen, die im Unterschied zu den vorigen mit nur 1 Laserpuls bei der Maximalfluenz von $5 \mathrm{~J} / \mathrm{cm}^{2}$ laserhydriert wurde. Sie weist im Unterschied zu der mit 16 Laserpulsen bestrahlten Probe eine Wasserstoff-Maximalkonzentration von 5 at\% auf, mit einem Ausläufer bis in eine Tiefe von $50 \mathrm{~nm}$.

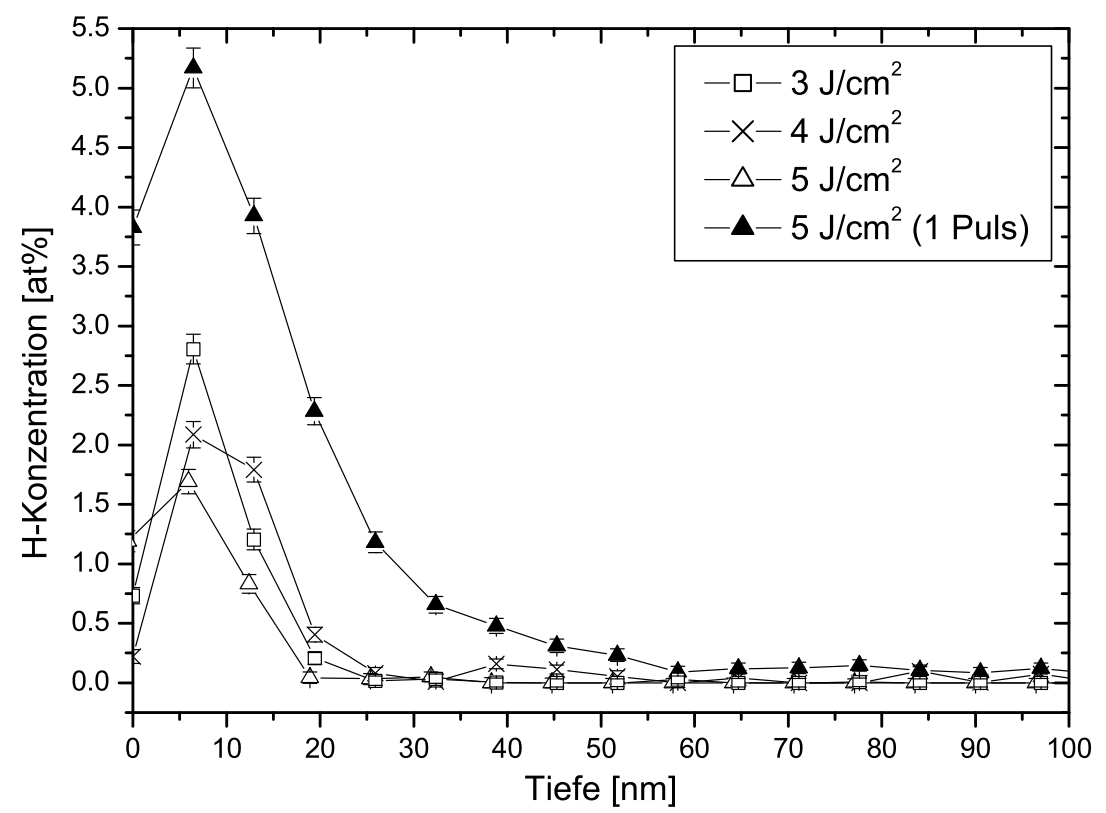

Abbildung 59: Wasserstofftiefenverteilung von Xe-amorphisierten Si-Proben, nach Laserhydrieren mit 16 Laserpulsen der Fluenz $3 \mathrm{~J} / \mathrm{cm}^{2}, 4 \mathrm{~J} / \mathrm{cm}^{2}, \mathrm{bzw} .5 \mathrm{~J} / \mathrm{cm}^{2}$ ( $p=2 \mathrm{bar}$ ). 


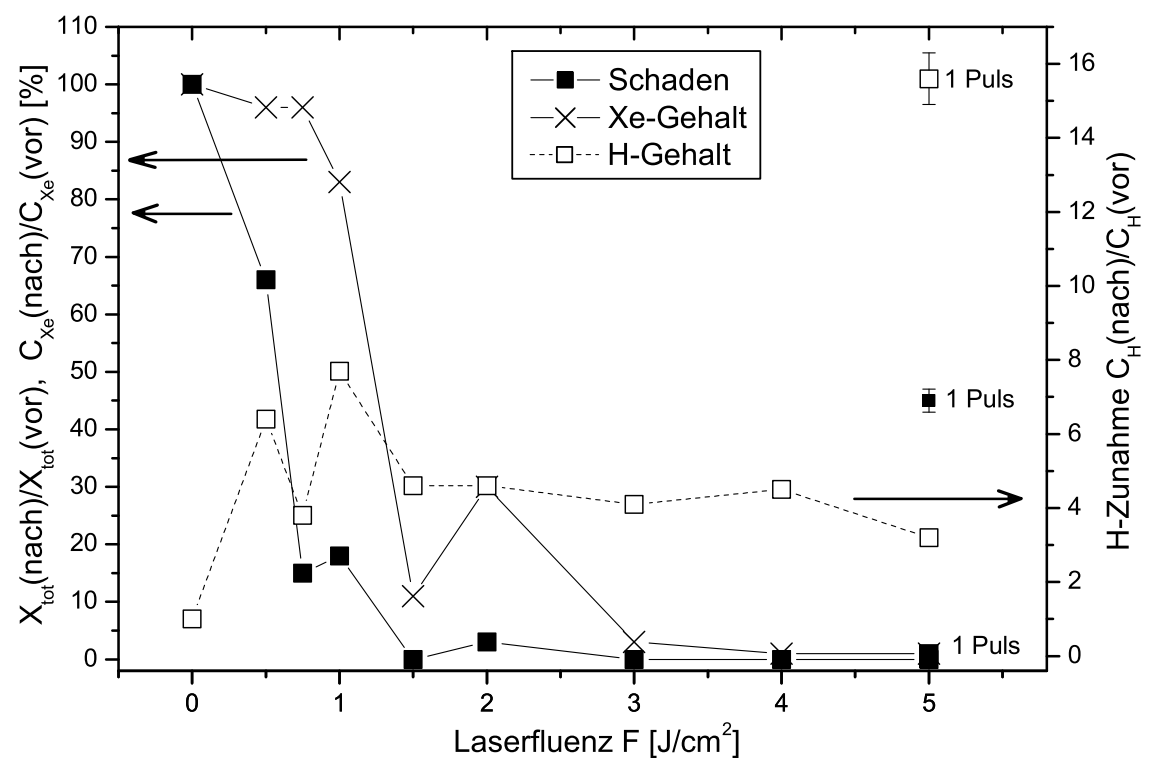

Abbildung 60: Relative Änderung von Xe-Gehalt, integraler Schädigung (linke Skala) und Wasserstoffgehalt (rechte Skala) als Funktion der Laserfluenz F. Die indizierten Symbole zeigen die Schädigung, den Xe-Gehalt und den H-Gehalt für eine Probe, die nur mit einem Laserpuls bestrahlt wurde. Für diese Probe sind typische Fehlerbalken angegeben.

Die wichtigsten Ergebnisse sind in Abb. 60 noch einmal zusammengefasst. In diesem Diagramm ist die integrierte Schädigung $\mathrm{X}_{\text {tot }}$ (nach) nach der Laserbestrahlung normiert auf die integrierte Schädigung vor der Laserbestrahlung $\mathrm{X}_{\text {tot }}$ (vor) als Funktion der Laserfluenz aufgetragen (linke Skala). Außerdem ist auch der auf den Anfangswert normierte Xe-Gehalt der Proben $\mathrm{C}_{X e}($ nach $) / \mathrm{C}_{X e}$ (vor) gezeigt (linke Skala). Zusätzlich kann anhand der rechten Skala die auf den Anfangsgehalt normierte Zunahme des integralen Wasserstoffgehalts der Proben $\mathrm{C}_{H}$ (nach) $/ \mathrm{C}_{H}$ (vor) nach dem Laserhydrieren abgelesen werden. Zuerst fällt auf, dass der integrale Wasserstoffgehalt der Proben nur sehr wenig ansteigt. Die Zunahme des Wasserstoffgehalts wurde an dieser Stelle auf den Mittelwert des Wasserstoffgehalts $\mathrm{C}_{H}$ (vor) $=4(1) \mathrm{H} / \mathrm{cm}^{2}$ von Xe-implantierten Si-Proben vor dem Laserhydrieren normiert. Lediglich die nur mit 1 Laserpuls der Fluenz $5 \mathrm{~J} / \mathrm{cm}^{2}$ bestrahlte Probe zeigt eine etwas stärkere Zunahme von $\mathrm{C}_{H}($ nach $) / \mathrm{C}_{H}($ vor $) \approx 16$. Die integrierte Schädigung sinkt schon bei kleiner Laserfluenz $\mathrm{F}=0.75 \mathrm{~J} / \mathrm{cm}^{2}$ auf unter $15 \%$ des ursprünglichen Wertes und nähert sich für höhere Fluenzen dem Wert Null, der einer vollständigen Rekristallisation entspricht. Der integrale Xe-Gehalt zeigt ein ganz ähnliches Verhalten, allerdings fällt der Wert des Xe-Gehaltes etwas langsamer als der der Schädigung. Zusammengefasst ergibt sich für das Laserhydrieren von a-Si damit folgendes Bild:

Schon bei einer Laserfluenz von $\mathrm{F}=1.5 \mathrm{~J} / \mathrm{cm}^{2}$ kommt es zur epitaktischen Rekristallisation der amorphen Deckschicht. Sie geht mit dem Ausdiffundieren des implantierten Xenons einher. Das Laserhydrieren führt erst ab einer Laserfluenz von $\mathrm{F}=2 \mathrm{~J} / \mathrm{cm}^{2} \mathrm{zu}$ einer Aufrauhung der a-Si- Probenoberfläche. Der effizienteste Bereich der Fluenz für die Wasserstoffaufnahme in die amorphe Schicht liegt bei $\mathrm{F} \approx 1 \mathrm{~J} / \mathrm{cm}^{2}$, eine Erhöhung der Energie führt zu einer geringeren Wasserstoffaufnahme. Dagegen wirkt sich eine Verringerung der Pulszahl, d.h. eine Konzentration der insgesamt eingebrachten Energie auf einen einzigen Puls, positiv auf den Hydrierprozess aus. 


\subsubsection{Laserhydrieren von H-implantiertem Silizium}

Ein Teil der untersuchten Si-Proben war vor dem Laserhydrieren mit Wasserstoffionen der Energie $\mathrm{E}=15 \mathrm{keV}$ implantiert worden. Durch die Implantation von Wasserstoffionen wurden zwei Dinge erreicht. Zum einen entstand durch die Implantation ein geschädigter Bereich, der vor und nach dem Laserhydrieren mittels RBS-Channeling untersucht wurde. Außerdem konnte anhand dieser Proben das Diffusionsverhalten einer bereits vorhandenen Wasserstoffkonzentration unter Laserbestrahlung bestimmt werden.

\section{Amorphisierung durch H-Implantation}

Um die Schädigung der Kristallstruktur infolge der Wasserstoff-Implantation festzustellen, wurden die Siliziumproben nach der Implantation mittels RBS-Channeling untersucht. Abbildung 61 zeigt die Schädigungsprofile für vier Si-Proben, die mit Wasserstoff-Molekül-Ionen der Energie $30 \mathrm{keV}$ implantiert wurden. Da die Wasserstoffmoleküle beim Auftreffen auf die Si-Oberfläche sofort dissoziieren, entspricht die Situation der einer Implantation der doppelten Menge an $\mathrm{H}^{+}$-Ionen mit der Energie $15 \mathrm{keV}$.

Die Implantation führt zu einem geschädigten Bereich in einer Tiefe von $200 \mathrm{~nm}$. Mit zunehmender Fluenz steigt der Maximalwert der normierten Schädigung $\chi_{\max }$ an, wobei sich die Tiefe des Schadensmaximums nicht ändert. In Abbildung 61 ist zu sehen, dass die Schicht bei der kritischen Fluenz $\phi_{k r i t}=9 \times 10^{16} \mathrm{H} / \mathrm{cm}^{2}$ vollständig amorphisiert wird $\left(\chi_{\max }=1\right)$. Im Unterschied zur Xe-Implantation bildet sich bei der WasserstoffImplantation ein schmaler amorphisierter Bereich aus, der bei Erreichen der kritischen Fluenz eine Breite von $80 \mathrm{~nm}$ hat. Bei der Implantation von Fluenzen $\phi>\phi_{k r i t}$ steigt diese Breite langsam an.

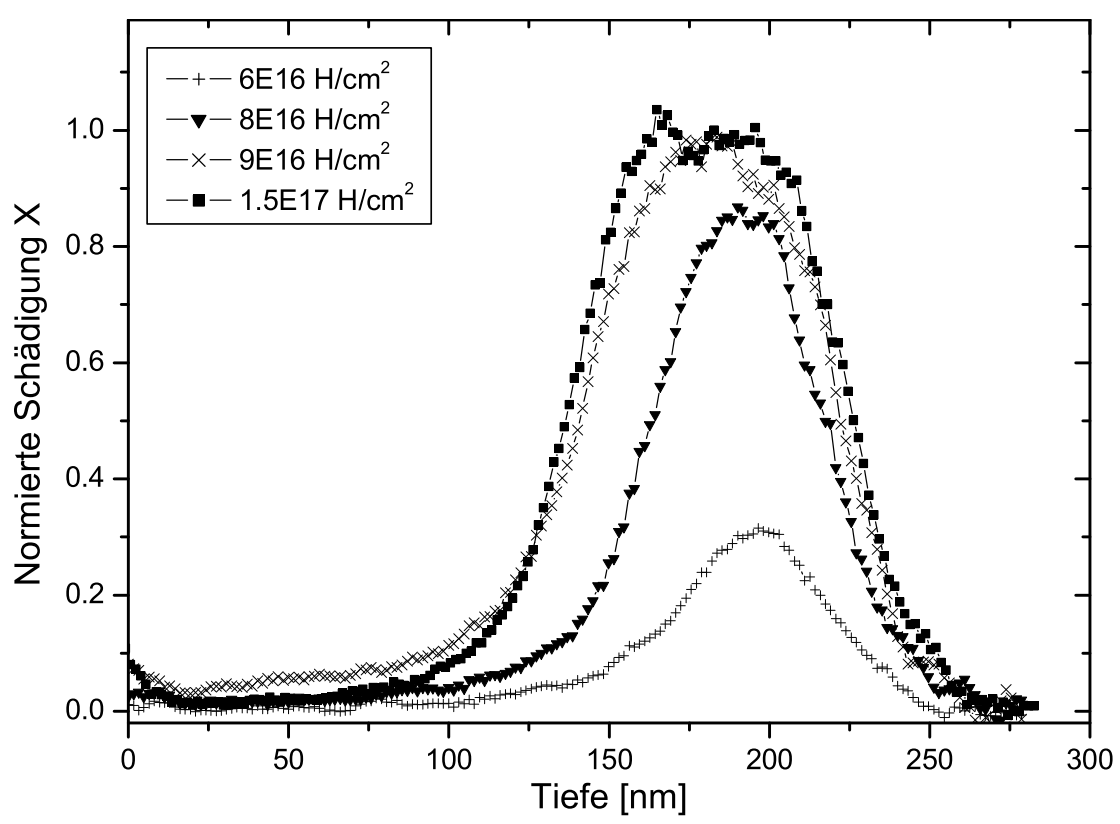

Abbildung 61: Schadensprofile von c-Si-Proben, die mit den angegebenen Wasserstoff-Fluenzen implantiert wurden. Die Energie der Protonen betrug $15 \mathrm{keV}$. 
Die Tiefenverteilung des implantierten Wasserstoffs wurde vor dem Laserhydrieren mittels NRA-Messungen bestimmt. Abbildung 62 zeigt das Wasserstofftiefenprofil zusammen mit der mittels RBS-Channeling bestimmten Schadensverteilung für eine Probe, die mit Wasserstoffionen der Fluenz $\phi=1.5 \times 10^{16} \mathrm{H} / \mathrm{cm}^{2}$ bestrahlt wurde. Bei der in Abbildung 62 ge-

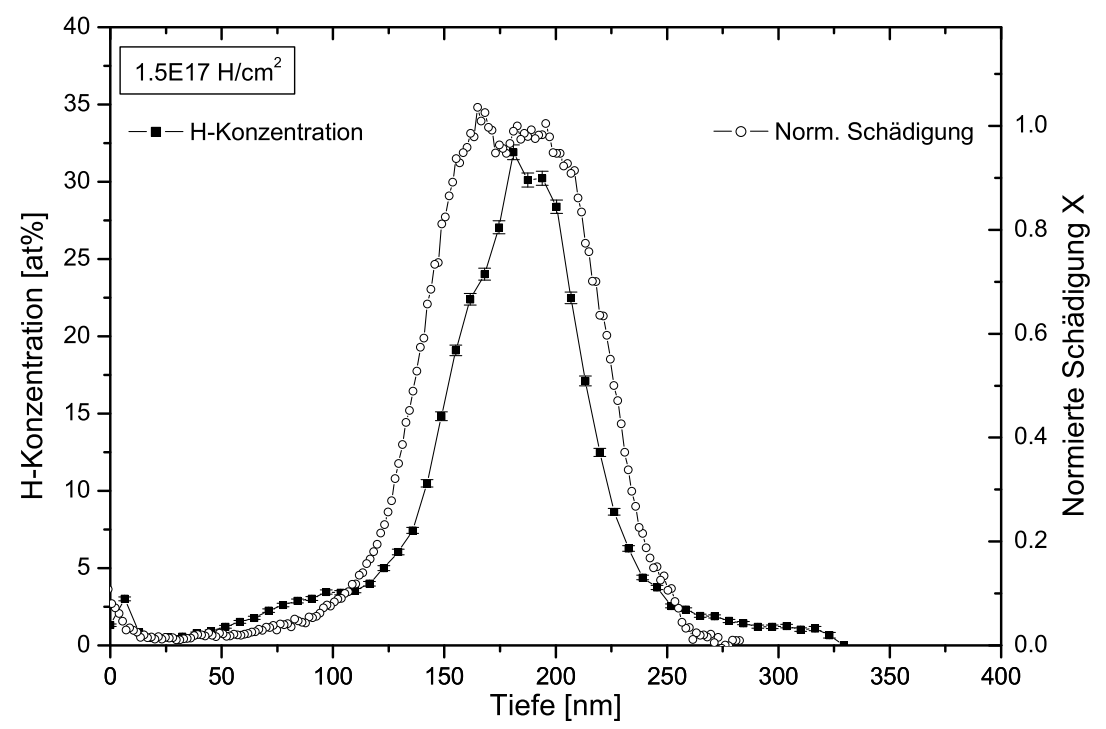

Abbildung 62: Wasserstoffprofil (gefüllte Quadrate) und Schadensprofil (offene Kreise) einer cSi-Probe, die mit Wasserstoffionen der Fluenz $\phi=1.5 \times 10^{16} \mathrm{H} / \mathrm{cm}^{2}$ implantiert wurde. Die Implantationsenergie der H-Ionen betrug $15 \mathrm{keV}$.

zeigten Probe, stimmt die Tiefe des Schadensmaximums und die Lage des Maximums des Wasserstoff-Implantationsprofils überein. Dieses Verhalten wurde bei allen WasserstoffImplantationen der vorliegenden Arbeit gefunden. Ursache für die Übereinstimmung von Wasserstoff- und Schadensprofil ist die in Kapitel 4.4 diskutierte fallenbeeinflusste Diffusion des Wasserstoffs. Während der Wasserstoff-Implantation kommt es zur Bildung von ausgedehnten geschädigten Bereichen nahe dem Ende der Ionenbahn. Die nachfolgend in die Nähe der Defekte implantierten H-Ionen lagern sich an die offenen Si-Bindungen an und sättigen diese ab [114. Da jedes eintreffende H-Ion jeweils mehrere Versetzungsstöße initiiert, stehen immer mehr Defekte als Ionen zur Verfügung. Durch Diffusion der H-Ionen zu diesen Defekten kommt es zu einer starken Anreicherung des Wasserstoffs im Bereich der Schädigung. Zusätzlich bewirken diese Bereiche hoher Wasserstoffkonzentration und großer Schädigung eine Störung der Kollisionskaskaden der nachfolgend implantierten Ionen.

Als weiteres Indiz für dieses Szenario der Wasserstoff-Implantation dient eine SRIM2000Simulation. Bei SRIM2000 [70] wird die Stoßkaskade der eintreffenden Ionen als eine Folge von binären Stößen betrachtet. Die Kaskade eines einzelnen Ions endet, sofern dessen Energie unter die Schwelle für eine Verlagerung eines weiteren Si-Atoms auch bei großem Stoßparameter sinkt. Damit bleiben interatomare Potenziale und daraus folgende Diffusionsprozesse des Implantats unberücksichtigt.

Abbildung 63 vergleicht die Ergebnisse aus einer SRIM2000-Simulation mit der Messung. Im oberen Teil ist das Schadensprofil einer Si-Probe abgebildet, die mit $15 \mathrm{keV}$ H-Ionen implantiert worden ist. Zusätzlich ist die Verteilung der deponierten Energiedichte $\mathrm{F}_{d}(\mathrm{z})$ 
angegeben, die sich mit Hilfe von Gl. 26 aus einer SRIM2000-Simulation ergibt. Insgesamt ist die Verteilung der deponierten Energiedichte $\mathrm{F}_{d}(\mathrm{z})$ sehr viel breiter $(\mathrm{FWHM=185 \textrm {nm } )}$ als die der gemessenen Schädigung (90(5) nm). Die Maxima der beiden Verteilungen liegen jedoch nur $45 \mathrm{~nm}$ auseinander, d.h. das Maximum der deponierten Energie wird von Gl. 26 in Verbindung mit den Werten aus SRIM2000 recht gut wiedergegeben. Auch die Schwerpunkte der gemessenen Verteilung (175(5) nm) und der Simulation (187 nm) stimmen sehr gut überein. Der Vergleich von gemessenem und simuliertem Implantationsprofil

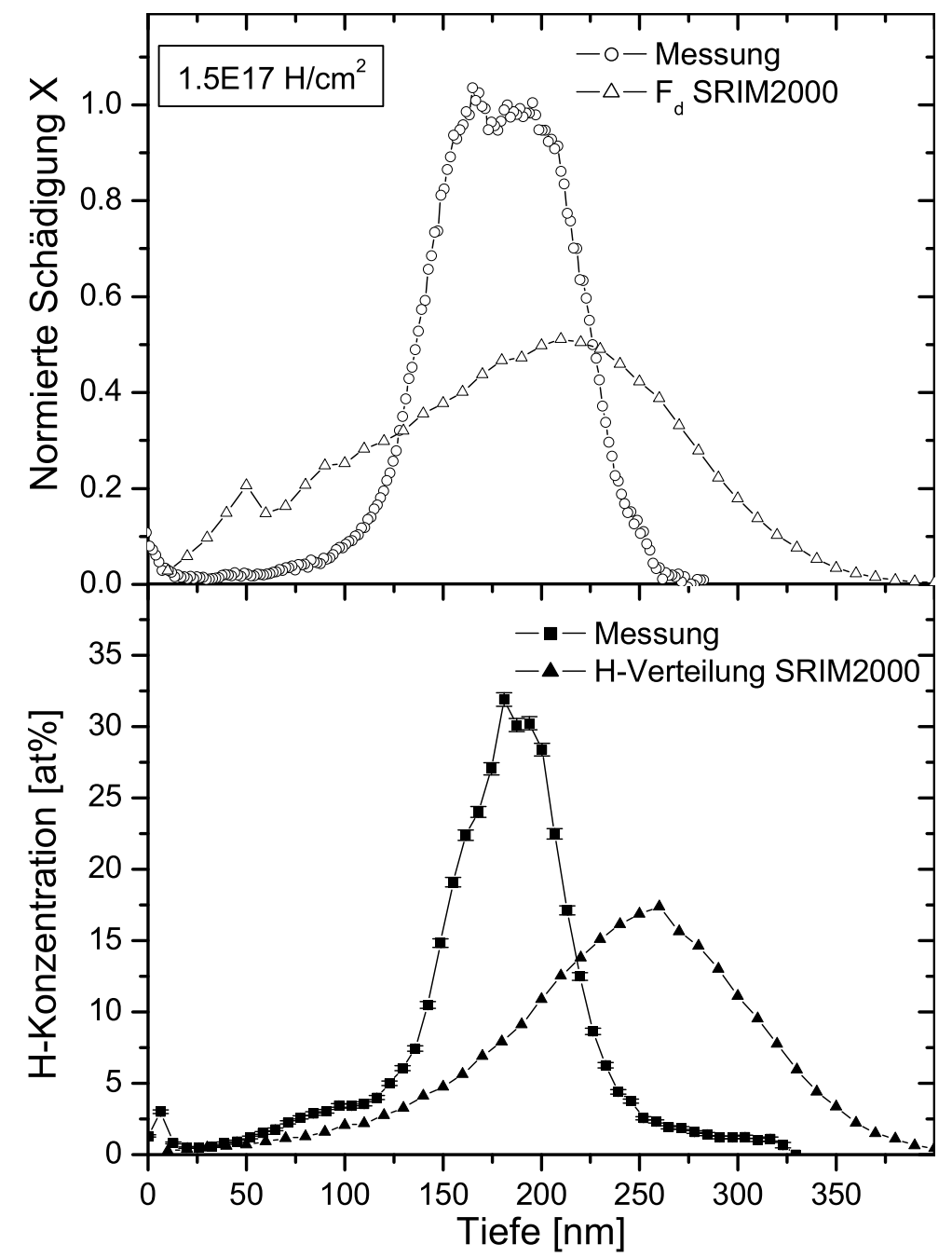

Abbildung 63: Vergleich von gemessenem und simuliertem Schadensprofil (oben) und gemessene bzw. simulierte Reichweiteverteilung der Wasserstoffionen einer c-Si-Probe, die mit $\phi=1.5 \times 10^{17} \mathrm{H} / \mathrm{cm}^{2}$ implantiert wurde. Die Energie der Protonen betrug $15 \mathrm{keV}$.

zeigt dagegen eine sehr viel schlechtere Übereinstimmung. Das Maximum der Wasserstoffkonzentration liegt bei der SRIM2000-Simulation mit $260 \mathrm{~nm}$ deutlich tiefer als bei der Messung 180(5) nm. Wie schon beim Schadensprofil wird auch die Breite des Implantationsprofils von der Simulation um einen Faktor zwei überschätzt: Experimentell ergibt sich eine Breite von 66 nm gegenüber 130 nm gemäß SRIM2000.

Auch für den Schwerpunkt der Wasserstoffverteilung ergibt die Simulation einen viel zu hohen Wert von $240 \mathrm{~nm}$, gegenüber dem experimentell bestimmten Wert von 177(5) nm. 
Der Vergleich von Simulation und Messdaten gibt einen deutlichen Hinweis darauf, dass der implantierte Wasserstoff nicht in der berechneten Implantationstiefe bleibt, sondern in das Maximum der Defekte diffundiert. Durch die Implantation der Proben bei StickstoffTemperatur wird die Diffusion während der Implantation zwar stark unterdrückt. Eine langsame Diffusion des Wasserstoffs ist während der Lagerung der Proben bei Raumtemperatur jedoch möglich. Zusammenfassend ergibt sich eine gute Übereinstimmung der Schwerpunkte der Schadensverteilung aus Messung und Simulation mit dem Schwerpunkt der Wasserstoffverteilung. Die Rückdiffusion des Wasserstoffs zu den in geringerer Tiefe liegenden Defekten und das daraus entstehende schmalere Wasserstofftiefenprofil erklären die Überschätzung der Ionenreichweite durch SRIM2000.

Die Bildung eines schmalen Defektbandes mit Wasserstoff-Agglomeraten wurde schon von einigen anderen Gruppen beobachtet [115, 116]. Diese sogenannten „Platelets“ sind Wasserstoffblasen mit sehr hohem Druck. Das Aufplatzen dieser Blasen nach dem Ausheizen der H-implantierten Proben bildet die Grundlage für den „Smart-Cut"-Prozess [117, 118, 119].

\section{Einfluss des NRA-Analysestrahls auf die Kristallstruktur}

Im Zusammenhang mit dem Wasserstoffnachweis an geschädigten Siliziumproben stellt sich die Frage, ob der NRA-Analysestrahl zu einer Veränderung der Kristallstruktur bei den hier untersuchten Si-Proben führt. Die Energie der ${ }^{15} \mathrm{~N}$-Ionen des Analysestrahls liegt bei über $6385 \mathrm{keV}$. SRIM2000 gibt für diesen Fall eine Reichweite der ${ }^{15} \mathrm{~N}$-Ionen von $5.2 \mu \mathrm{m}$ an. Damit liegen auch die Strahlenschäden, die die Proben während der NRAAnalyse erfahren, in einer wesentlich größeren Tiefe, als der hier mittels RBS-Channeling untersuchte Bereich von 0-400 nm. Abbildung 64 zeigt Schadensprofile für zwei Siliziumproben, die mit H-Ionen der Fluenz $\phi=10^{17} \mathrm{H} / \mathrm{cm}^{2}$ (oben) bzw. $\phi=6 \times 10^{16} \mathrm{H} / \mathrm{cm}^{2}$ (unten) bestrahlt wurden. Die Schadensprofile vor und nach der NRA-Analyse zeigen nur geringe Abweichungen. Die wichtigsten Parameter sind in Tabelle 12 zusammengefasst.

\begin{tabular}{c|c|c|c|c}
$\begin{array}{c}\phi(\mathrm{H}) \\
{\left[\mathrm{H} / \mathrm{cm}^{2}\right]}\end{array}$ & $\begin{array}{c}\text { Zeitpunkt } \\
\text { d. Messung }\end{array}$ & $\begin{array}{c}\mathrm{X}_{\text {tot }} \\
{\left[10^{15} \mathrm{at} / \mathrm{cm}^{2}\right]}\end{array}$ & $\begin{array}{c}\bar{z}_{\text {dam }} \\
{[\mathrm{nm}]}\end{array}$ & $\begin{array}{c}\mathrm{b}_{\text {dam }} \\
{[\mathrm{nm}]}\end{array}$ \\
\hline \hline $5 \mathrm{E} 16$ & vor NRA & $51(2)$ & $184(4)$ & $60(3)$ \\
\hline $5 \mathrm{E} 16$ & nach NRA & $71(2)$ & $194(4)$ & $60(3)$ \\
\hline $1 \mathrm{E} 17$ & vor NRA & $483(19)$ & $173(4)$ & $89(3)$ \\
\hline $1 \mathrm{E} 17$ & nach NRA & $448(18)$ & $183(4)$ & $81(3)$ \\
\hline
\end{tabular}

Tabelle 12: Vergleich der Schadensprofile vor und nach der NRA-Analyse: Fluenz der implantierten H-Ionen $\phi(H)$, integrierte Schädigung $X_{\text {tot }}$, Schwerpunkt des Schadensprofils $\bar{z}_{\text {dam }}$ und Halbwertsbreite $b_{d a m}$ 


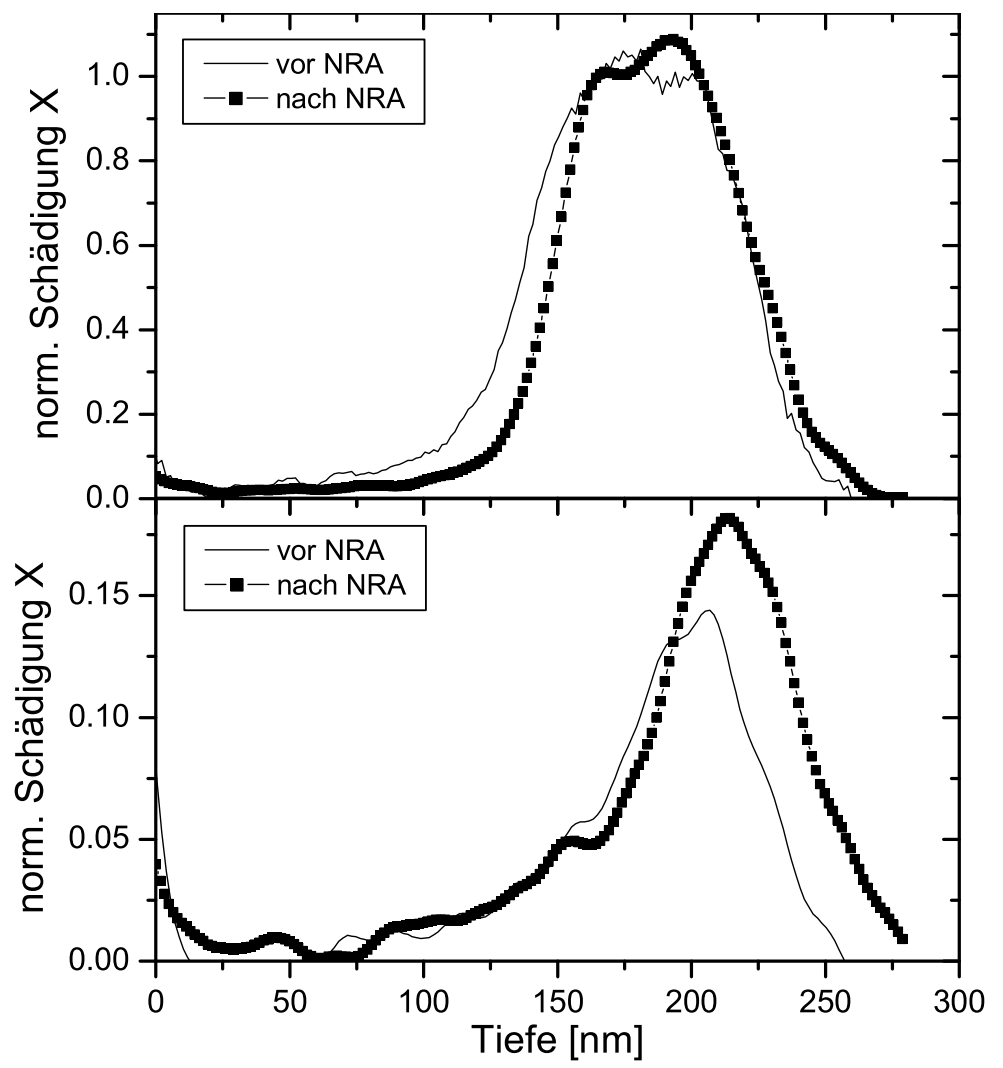

Abbildung 64: RBS-Channeling-Spektren vor und nach der NRA-Analyse mittels der ${ }^{1} H\left({ }^{15} \mathrm{~N}, \alpha \gamma\right){ }^{12} \mathrm{C}$-Kernreaktion bei $E_{R}=6385 \mathrm{keV}$. Oben: Schadensprofil $\chi_{n o r m}(z)$ für eine SiProbe, die mit H-Ionen der Fluenz $\phi=10^{17} \mathrm{H} / \mathrm{cm}^{2}$ implantiert worden war. Unten: Schadensprofil $\chi_{\text {norm }}(z)$ für eine Si-Probe, die mit H-Ionen der Fluenz $\phi=6 \times 10^{16} \mathrm{H} / \mathrm{cm}^{2}$ implantiert worden war (Schädigung ebenfalls deutlich geringer).

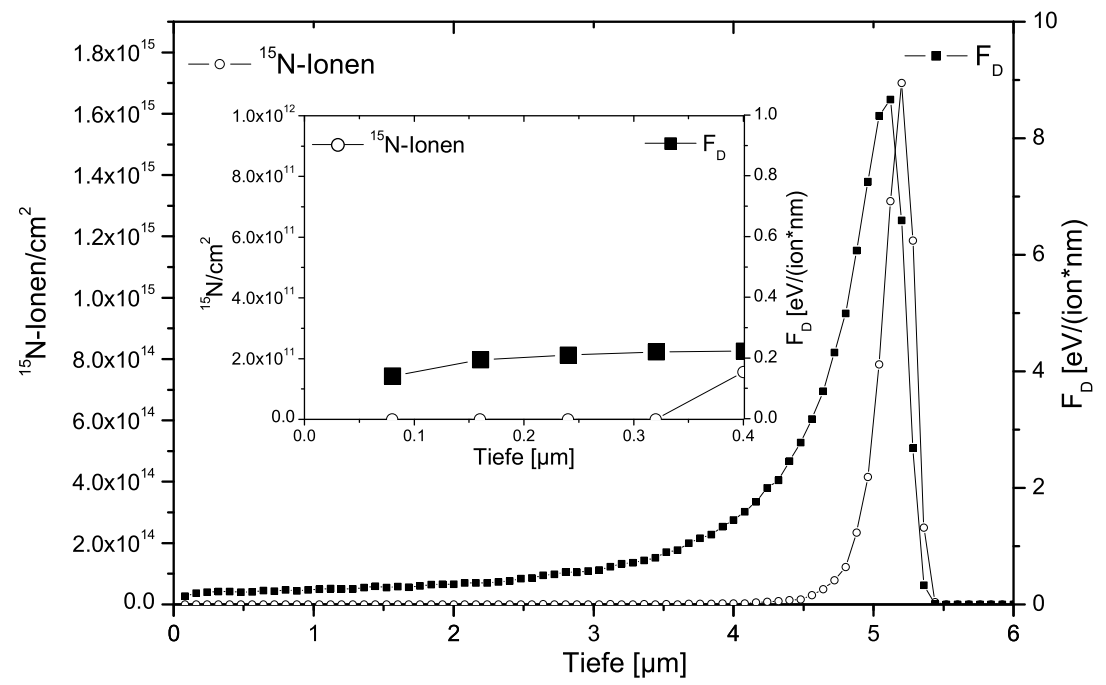

Abbildung 65: Implantationsprofil und deponierte Energiedichte $F_{D}$ aus einer Srim2000Simulation für 6.4 MeV ${ }^{15} \mathrm{~N}$-Ionen in Si. Einsatz: Vergrößerung im Tiefenbereich 0-400 nm 
Die Probe, die mit der hohen Fluenz von $\phi=10^{17} \mathrm{H} / \mathrm{cm}^{2}$ (Abb. 64 oben) implantiert worden war und deren Maximalwert der Schädigung $\chi_{\max }=1$ erreicht, wird durch den Analysestrahl kaum verändert. Bei der ursprünglich nur wenig geschädigten Probe (Abb. 64 unten) erhöht sich der Maximalwert der normierten Schädigung jedoch von $\chi_{\max }($ vor $)=0.14$ auf $\chi_{\max }($ nach $)=0.18$. Wie Tabelle 12 zeigt, verschiebt sich infolge der NRA-Analyse die Lage des Schwerpunktes der Schadensverteilung $\bar{z}_{\text {dam }}$ bei beiden Proben um $10 \mathrm{~nm}$ in das Probenvolumen.

Die in Abb. 65 dargestellte SRIM2000-Simulation zeigt das Implantationsprofil und die deponierte Energiedichte für die Implantation von $\phi=5 \times 10^{17} 15 \mathrm{~N} / \mathrm{cm}^{2}$ in Silizium. Zur Berechnung der deponierten Energidichte $\mathrm{F}_{D}$ wurde wieder Gl. 26 verwendet.

Das Maximum des ${ }^{15} \mathrm{~N}$-Implantationsprofils liegt gemäß der Simulation in einer Tiefe von $5.2 \mu \mathrm{m}$ und das Maximum der deponierten Energiedichte bei $5.1 \mu \mathrm{m}$. Der Einsatz in Abb. 65 stellt den Bereich bis $\mathrm{z}=400 \mathrm{~nm}$, in dem die Schadensprofile aus Abb. 64 liegen, noch einmal vergrößert da. Es zeigt sich, dass in Übereinstimmung mit den experimentellen Schädigungsprofilen auch aus der Simulation eine von Null verschiedene deponierte Energiedichte folgt.

\section{Oberflächenrauigkeit}

Die Untersuchungen zur Oberflächenrauigkeit der Xe-amorphisierten Proben in Abs. 6.3.1 haben gezeigt, dass die laserinduzierte Rekristallisation und das Ausdiffundieren des Xenons keine direkten Auswirkungen auf die Rauigkeit der Probenoberfläche hat. Eine starke Zunahme der Oberflächenrauigkeit wurde nur dann festgestellt, wenn die Laserfluenz zum Zünden eines Plasmas an der Probenoberfläche ausreichte. In Abb. 66 ist die Rauigkeit der Probenoberfläche von H-implantierten Si-Proben als Funktion der Laserfluenz dargestellt.

Allgemein fällt auf, dass die Laserbestrahlung bei der Laserfluenz $F=0.5 \mathrm{~J} / \mathrm{cm}^{2}$ einen geringeren Einfluss auf die Rauigkeit der Probenoberfläche hat als die Bestrahlung mit $\mathrm{F}=1 \mathrm{~J} / \mathrm{cm}^{2}$. Die zum Teil recht unterschiedlichen Fehler bei der Rauigkeitsmessung rühren einzig aus der Statistik her. Jede Probenoberfläche wurde viermal vermessen. War die Probenoberfläche bei dieser Messung stark inhomogen, so ergibt die Mittelwertbildung einen großen Fehler. Diese Inhomogenitäten rühren von der starken Ortsabhängigkeit der Intensität des benutzten Laser-Rohstrahls her. Sie sind auf der Probenoberfläche nach der Bestrahlung zum Teil deutlich erkennbar.

Ein Beispiel für eine solch inhomogen modifizierte Probenoberfläche zeigt das in Abb. 67 dargestellte Foto einer Probenoberfläche nach dem Laserhydrieren. Die hier gezeigte Probe wurde zunächst mit Wasserstoffionen einer Fluenz von $\phi=1.5 \times 10^{17} \mathrm{H} / \mathrm{cm}^{2}$ bestrahlt und dann bei einem Wasserstoffdruck von 2 bar mit 1 Laserpuls der Laserfluenz $\mathrm{F}=0.5 \mathrm{~J} / \mathrm{cm}^{2}$ laserhydriert. Deutlich ist im mittleren Bereich der Probe der helle Laserfleck erkennbar. Die Bestimmung der Oberflächenrauigkeit wurde im Gebiet des Laserflecks durchgeführt, das von wellenartigen Strukturen durchzogen ist. Im Unterschied zu den Xeamorphisierten Proben des vorigen Abschnitts ändert sich die Oberflächenmorphologie der H-implantierten Proben schon bei einer Bestrahlung mit der Laserfluenz $\mathrm{F}=1 \mathrm{~J} / \mathrm{cm}^{2}$ sehr stark. Der Grund dafür ist das Aufplatzen der sog. „Platelets“, die tiefe Krater auf der Probenoberfläche hinterlassen [120]. 


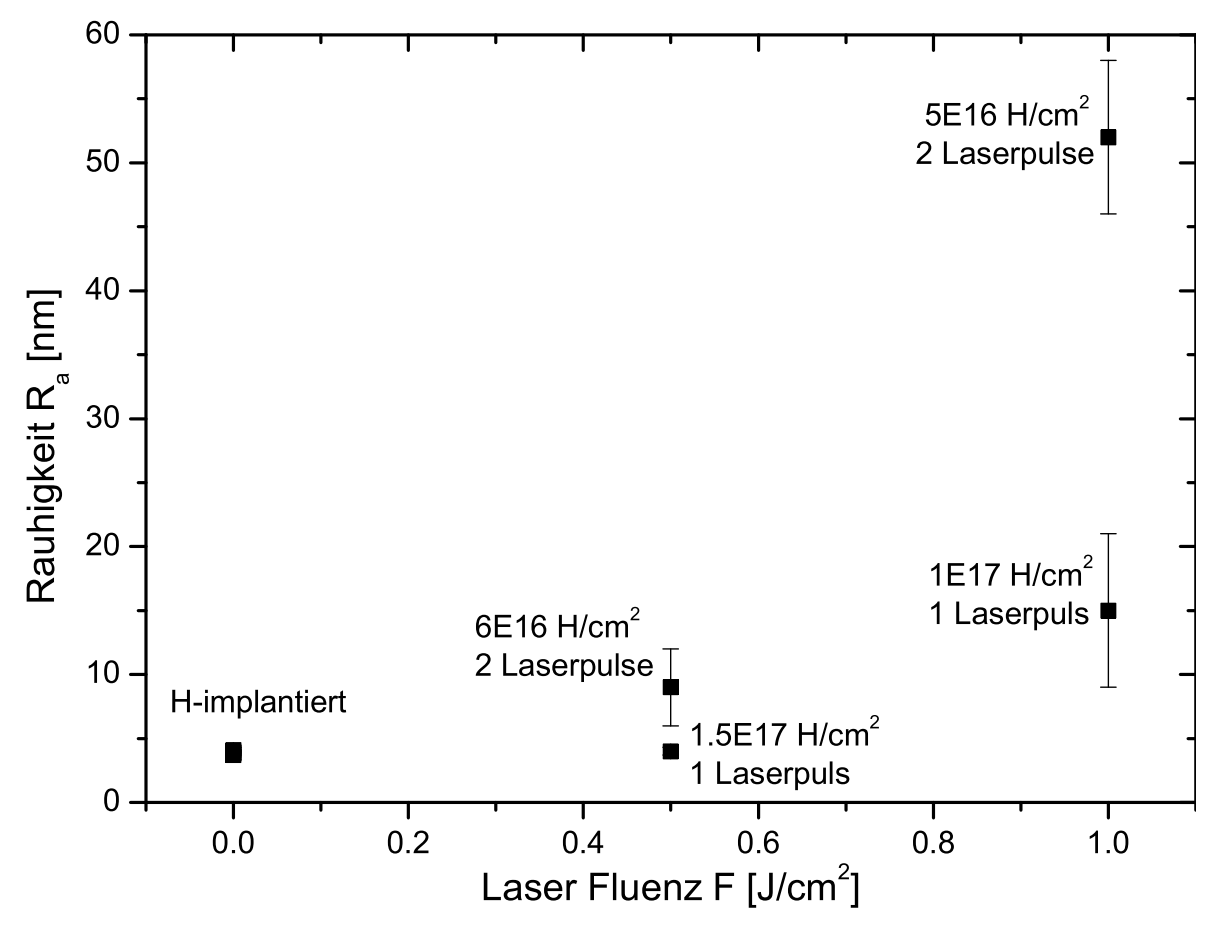

Abbildung 66: Oberflächenrauigkeit von H-implantierten Si-Proben, nach Bestrahlung mit 1 bzw. 2 Laserpulsen

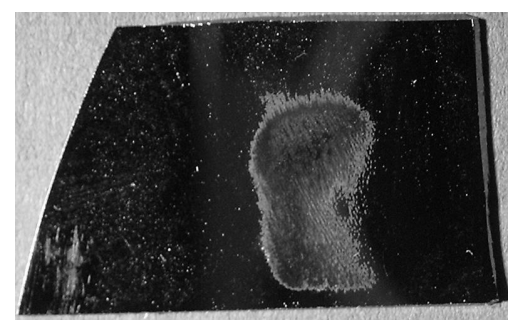

Abbildung 67: Foto einer laserhydrierten H-implantierten $\left(\phi=1.5 \times 10^{17} \mathrm{H} / \mathrm{cm}^{2}\right)$ Probe, die mit 1 Laserpuls der Fluenz $F=0.5 \mathrm{~J} / \mathrm{cm}^{2}$ bestrahlt wurde. Deutlich ist in der Probenmitte der helle Bereich des Laserflecks zu sehen. Die Nutzung des Laser-Rohstrahls führt zu starken Inhomogenitäten der Strahlintensität auf der Probenoberfläche. 


\section{Wasserstoff- und Schadensprofile nach dem Laserhydrieren}

Um herauszufinden, wie stark sich der Wasserstoffgehalt durch das Laserhydrieren von Proben verändert, die bereits vor der Laserbehandlung durch H-Implantation eine hohe Wasserstoffkonzentration aufweisen, wurden NRA-Messungen vor und nach dem Laserhydrieren durchgeführt. Zusätzlich wurde vor und nach dem Laserhydrieren die Schädigung der Kristallstruktur mittels RBS-Channeling bestimmt. Abbildung 68 zeigt das Ergebnis
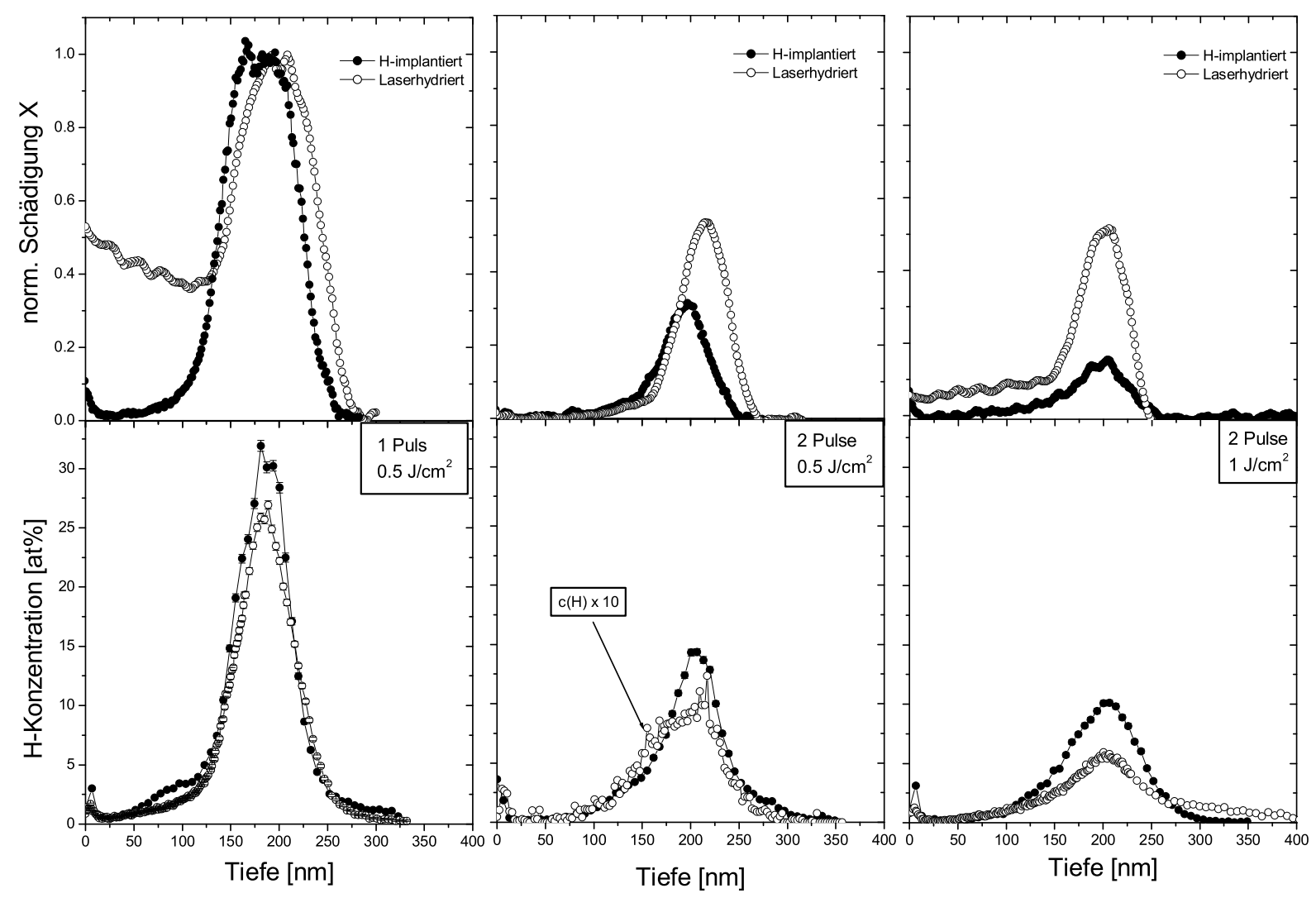

Abbildung 68: Schadensverteilung (oben) und Wasserstofftiefenprofil (unten) für H-implantierte Si-Proben vor (gefüllte Symbole) und nach dem Laserhydrieren (offene Symbole) mit der Fluenz $F=0.5 \mathrm{~J} / \mathrm{cm}^{2}$. Links: H-implantierte Probe mit $\phi=1.5 \times 10^{17} \mathrm{H} / \mathrm{cm}^{2}, 1$ Laserpuls. Rechts: $\mathrm{H}$ implantierte Probe mit $\phi=6 \times 10^{16} \mathrm{H} / \mathrm{cm}^{2}$, 2 Laserpulse.

für drei Proben, die nach der Wasserstoff-Implantation mit der Laserfluenz $\mathrm{F}=0.5 \mathrm{~J} / \mathrm{cm}^{2}$ (links und mitte) und $\mathrm{F}=1 \mathrm{~J} / \mathrm{cm}^{2}$ (rechts) bei 2 bar Wasserstoffdruck laserhydriert wurden. Die Probe im linken Teilbild wurde mit 1 Laserpuls, die Proben im mittleren und rechten Teilbild wurden mit 2 Laserpulsen bestrahlt. Die mit der hohen Ionenfluenz $\phi=1.5 \times 10^{17} \mathrm{H} / \mathrm{cm}^{2}$ implantierte Probe im linken Teilbild zeigt nach der Laserbestrahlung eine starke Schädigung, die bis zur Probenoberfläche reicht. Insgesamt hat die integrale Schädigung dieser Probe durch die Laserbestrahlung um 60\% zugenommen. Das Wasserstofftiefenprofil (links unten) zeigt dagegen nur geringe Veränderungen. Form und Lage des Wasserstoffprofils wurden durch die Laserbestrahlung nicht verändert. Der Wasserstoffgehalt der Probe hat um 19\% abgenommen. Ganz ähnlich ist das Ergebnis für die mit der niedrigeren Ionenfluenz $\phi=6 \times 10^{16} \mathrm{H} / \mathrm{cm}^{2}$ implantierte Probe im mittleren Teilbild, 
die mit 2 Laserpulsen bestrahlt wurde. Die Schädigung hat durch die Laserbestrahlung um $68 \%$ zugenommen, während der Wasserstoffgehalt sogar um 92\% abgenommen hat. Das Wasserstoffprofil dieser Probe nach dem Laserhydrieren ist zur besseren Übersicht zehnfach vergrößert dargestellt. Die integrale Schädigung der im rechten Teilbild von Abb. 68 dargestellten Probe, die mit der Ionenfluenz $\phi=5 \times 10^{16} \mathrm{H} / \mathrm{cm}^{2}$ implantiert worden war, hat durch die Laserbestrahlung sehr stark um 300\% zugenommen. Außerdem weist das Schadensprofil einen Ausläufer in Richtung der Probenoberfläche auf, der bereits bei der Bestrahlung mit $\mathrm{F}=0.5 \mathrm{~J} / \mathrm{cm}^{2}$ im linken Teilbild beobachtet wurde. Der Wasserstoffgehalt hat durch die Laserbestrahlung mit 2 Laserpulsen um 30\% abgenommen.

Im Unterschied zu den Ergebnissen beim Laserhydrieren von Xe-amorphisiertem Silizium führt die Laserbestrahlung von H-amorphisiertem Silizium also zu einer Zunahme der Schädigung. Durch Laserhydrieren wird der Wasserstoffgehalt hier nicht erhöht, sondern ein Teil des zuvor in der Probe gespeicherten Wasserstoffs verlässt das Silizium. Das Ausgasen aus der Probenoberfläche wird dabei durch die beobachteten oberflächennahen Defekte begünstigt.

Bei der Laserbestrahlung mit der relativ geringen Laserfluenz von $\mathrm{F}=0.5 \mathrm{~J} / \mathrm{cm}^{2}$ kommt es zu einem Ausdiffundieren des in der Probe vorhandenen Wasserstoffs. Anders als im Fall der Xe-Tiefenprofile wird hier jedoch keine Bewegung des Implantationsprofils hin zur Probenoberfläche beobachtet. Die Position der maximalen Wasserstoffkonzentration bleibt vielmehr nach der Laserbestrahlung gleich. 


\section{Zusammenfassung und Ausblick}

\subsection{Laserhydrieren von Titan}

In Abs. 6.1 konnte gezeigt werden, dass das Laserhydrieren von Titan gelingt. Bis in eine Tiefe von mindestens $400 \mathrm{~nm}$ wird Wasserstoff in das Probenvolumen eingebracht. Die Wasserstoffkonzentration nähert sich mit zunehmender Tiefe dem Grenzwert von $\mathrm{c}(\mathrm{H})=44-47$ at\%. Dieser Grenzwert wird unabhängig von der Pulszahl und dem Druck des Wasserstoffgases erreicht. Im oberflächennahen Bereich bis etwa $170 \mathrm{~nm}$ ist die Wasserstoffkonzentration dagegen deutlich niedriger. Hier muss davon ausgegangen werden, dass die Oberfläche beim Erstarrungsprozess als Senke für den in der Schmelze vorhandenen Wasserstoff wirkt. Da die Diffusivität von Wasserstoff in der Titanschmelze größer ist als im festen Titan, wird mit dem Erstarrungsvorgang stets Wasserstoff an der Probenoberfläche ausgasen. Wie die XRD-Spektren zeigen, bildet sich beim Laserhydrieren $\mathrm{TiH}_{2}$. Dieses Dihydrid wird bei Temperaturen oberhalb $720 \mathrm{~K}$ instabil und zerlegt sich in Titan und Wasserstoff. Da die Probenoberfläche in jedem Fall am längsten aufgeschmolzen bleibt, ist hier mit der stärksten Wasserstoffabnahme zu rechnen.

Die höchste Wasserstoffaufnahme wurde für die Bestrahlungen mit dem geringsten Wasserstoffdruck und bei der kleinsten verwendeten Pulszahl gemessen. Dies lässt einen Rückschluss auf die Entstehung des $\mathrm{TiH}_{2}$ zu: Je geringer der Kammerdruck bei den Laserbestrahlungen ist, desto größer ist die Menge des ablatierten Materials [107]. Im hier untersuchten Fall ist der Wasserstoffeintrag also besonders groß, wenn viel Material ablatiert wird. Dies spricht dafür, dass sich das $\mathrm{TiH}_{2}$ aus ablatierten Ti-Ionen bildet, die sich beim Abkühlen aus der Plasmaphase an Wasserstoffionen binden. Dieser $\mathrm{TiH}_{2}$-Fall-out setzt sich auf der abkühlenden Titan-Oberfläche als gräulich-schwarzer Film ab. Der niedrigere Wasserstoffgehalt bei höheren Pulszahlen lässt sich dann als fortschreitende Dekomposition des bereits vorhandenen $\mathrm{TiH}_{2}$ erklären.

Zur Untermauerung dieses Szenarios für die Bildung von $\mathrm{TiH}_{2}$ wären Experimente zum Laserhydrieren mit kleinerer Fluenz des Lasers geeignet. Aufgrund der niedrigeren Fluenz würde auch die Zeit herabgesetzt, während der die Titanoberfläche aufgeschmolzen vorliegt. Damit würden Diffusionsprozesse des Wasserstoffs in der Schmelze stark unterdrückt. Wenn die hier angegebene Modellvorstellung der Bildung des $\mathrm{TiH}_{2}$ im reaktiven Fall-out zutrifft, sollte die Menge des eingebrachten Wasserstoffs unabhängig sein von der Fluenz des Lasers. Die Wasserstoffdiffusion während der Bestrahlung liesse sich ebenfalls durch eine Kühlung der Proben beim Laserhydrieren unterdrücken.

Experimente zur Abhängigkeit der Wasserstoffaufnahme von der Laserfluenz wären auch aus einem zweiten Grund sinnvoll. Alle hier gezeigten Ergebnisse wurden bei der festen Laserfluenz $\mathrm{F}=4 \mathrm{~J} / \mathrm{cm}^{2}$ durchgeführt, die zum Zünden eines Plasmas ausreicht. Eine Bestrahlung mit niedrigerer Laserfluenz knapp oberhalb der Plasmaschwelle hätte zur Folge, dass weniger Material an der Probenoberfläche abgetragen wird und somit weniger FallOut für die Reaktion mit dem Wasserstoff zur Verfügung steht. Das Zünden des Plasmas muss jedoch auch hier gewährleistet sein, damit genügend Energie zur Dissoziation des molekularen Wasserstoffs bereitsteht. Eine starke Erhöhung der Laserfluenz sollte dann umgekehrt den Wasserstoffeintrag erhöhen. 


\subsection{Laserhydrieren von Silizium}

Beim Laserhydrieren von amorphem und kristallinem Silizium konnten insgesamt nur sehr geringe Mengen Wasserstoff in den Proben nachgewiesen werden. Während die Titanproben nach dem Laserhydrieren eine mit der Tiefe zunehmende Wasserstoffkonzentration zeigten, wurde für die Siliziumproben nur an der Oberfläche eine geringfügige Erhöhung des Wasserstoffgehalts festgestellt. Für laserhydriertes kristallines Silizium wurde eine größere Wasserstoffaufnahme als für amorphes Silizium gemessen.

\section{Kristallines Silizium}

Die Oberflächenrauigkeit der laserhydrierten c-Si-Proben nimmt oberhalb der Laserfluenz von $4 \mathrm{~J} / \mathrm{cm}^{2}$ um einen Faktor 10 auf den Wert $\mathrm{R}_{a} \approx 230 \mathrm{~nm} \mathrm{zu}$. Dies ist gleichzeitig die Schwellenfluenz, oberhalb derer ein Plasma an der Si-Oberfläche beobachtet wurde. Die Simulation der Oberflächentemperatur ergibt, dass bei der Bestrahlung mit $\mathrm{F}=4 \mathrm{~J} / \mathrm{cm}^{2}$ für kurze Zeit (ca. 50 ns) die Siedetemperatur des Siliziums überschritten wird. Es stehen also zusätzlich zu den energiereichen Elektronen auch Si-Ionen an der Probenoberfläche für die Plasmabildung zur Verfügung. Durch diese oberflächennahe Wärmequelle kann auch der Wasserstoff dissoziieren.

Die Aufschmelztiefe beträgt laut Simulation für diesen Fall $1.2 \mu \mathrm{m}$. Dies ist deutlich mehr, als die mittels RBS-Channeling ermittelte Schädigungstiefe der Siliziumproben nach der Laserbestrahlung von ca. $30 \mathrm{~nm}$. Während des Abkühlprozesses kommt es folglich zur epitaktischen Rekristallisation. Die Rekristallisation geschieht jedoch nicht vollständig, sondern vielmehr bleibt an der Probenoberfläche ein geschädigter Bereich zurück. Die Tiefe des geschädigten Bereichs nimmt dabei mit der Laserfluenz zu. Nur in diesem Bereich konnte auch ein erhöhter Wasserstoffgehalt nach dem Laserhydrieren festgestellt werden. Die RBS-Channeling Messungen der laserhydrierten Si-Proben ergaben eine maximale normierte Schädigung von $\chi_{\max } \leq 0.65$ an der Probenoberfläche. Diese Defekte können als Senke für Wasserstoffatome beim Erstarrungsprozess der aufgeschmolzenen Oberflächenschicht wirken. Außerdem zeigte sich, dass die Menge des pro Defekt gespeicherten Wasserstoffs nicht konstant ist. Statt dessen lagert sich bei zunehmender Laserfluenz immer mehr Wasserstoff an den bereits vorhandenen Defekten an.

Die Ursache für die unvollständige Rekristallisation der laserhydrierten Si-Proben ist vor

allem in der Einwirkung des Plasmas zu sehen. Die Schadensprofile zeigten eine starke Zunahme der Schädigung nahe der Probenoberfläche, sofern an der Probenoberfläche ein Plasma zündete. Ein zweiter Effekt kann ebenfalls zu einer gestörten Probenoberfläche führen. Es ist bekannt, dass kristallines Silizium durchaus in der amorphen Phase erstarren kann. Liu et al. fanden die Bildung einer amorphen Schicht durch Bestrahlung von kristallinem Silizium mit 30 ps-Laserpulsen [121]. Da die Temperaturgradienten innerhalb der mehreren 10 nm-dicken geschmolzenen Schicht sehr groß sind, geschieht die Rekristallisation sehr schnell. Spaepen und Turnbull [122] zeigen, dass die Laser-Rekristallisation einer amorphen Schicht auf einem kristallinen Substrat für beinahe alle Materialien, also auch für das kovalent gebundene Silizium, grenzflächen-limitiert ist. Damit bezeichnen sie die Eigenschaft, dass die Temperatur $\mathrm{T}_{c / a}$ der Grenzfläche kristallin/amorph immer nahe der Temperatur $\mathrm{T}$ des umgebenden Materials liegt. Daraus folgern sie, dass die Tem- 
peratur der Grenzschicht $\mathrm{T}_{c / a}$ stark von der Schmelztemperatur $\mathrm{T}_{\text {melt }}$ abweichen kann und im Extremfall großer Temperaturgradienten unter die Schmelztemperatur $\mathrm{T}_{\text {amelt }}$ der amorphen Phase fallen kann. In diesem Fall ist es thermodynamisch möglich, dass sich die amorphe Phase aus der Schmelze bildet. Topologische Störungen werden dann nicht mehr korrigiert, sondern wachsen weiter zu einer amorphen Schicht. Bloembergen [123] hat berechnet, dass sich durch die Erstarrung der Siliziumschmelze in die amorphe Phase eine bis zu $20 \mathrm{~nm}$ dicke amorphe Schicht auf laserbestrahltem kristallinem Silizium bilden kann.

Das Vorhandensein von Wasserstoff in den laserhydrierten kristallinen Siliziumproben wird in der vorliegenden Arbeit auf die Anlagerung des Wasserstoffs an oberflächennahe Defekte zurückgeführt. Im Fall des im vorigen Abschnitt behandelten Laserhydrierens von Titan konnte mittels XRD-Messungen die Bildung einer $\mathrm{TiH}_{2}$-Phase nachgewiesen werden. Diese wurde für den erhöhten Wasserstoffgehalt der Proben nach dem Laserhydrieren verantwortlich gemacht. Prinzipiell ist beim Laserhydrieren von Silizium die Bildung von Silan (z.B. Monosilan $\mathrm{SiH}_{4}$ ) denkbar. Die Si-H-Bindungen des Silans sind jedoch, aufgrund der negativen Polarisierung der H-Atome in der Bindung, äußerst instabil. Daher kann in Anbetracht der hohen Temperaturen die Bildung von Silan während des Laserhydrierens ausgeschlossen werden.

Abschließend ergibt sich zur Erklärung für den erhöhten Wasserstoffgehalt der kristallinen Siliziumproben nach dem Laserhydrieren folgendes Szenario:

Die Bestrahlung mit Laserfluenzen $\mathrm{F}>4 \mathrm{~J} / \mathrm{cm}^{2}$ führt zur Bildung eines Plasmas an der Probenoberfläche. Gemäß der in Abb. 69 gezeigten Simulation der Aufschmelztiefe als Funktion der Zeit, schmilzt die Probe bei der Bestrahlung mit $\mathrm{F}=5 \mathrm{~J} / \mathrm{cm}^{2}$ innerhalb der ersten $100 \mathrm{~ns}$ nach dem Einsetzen des Lasers bis in eine Tiefe von ca. $1.3 \mu \mathrm{m}$ auf. Durch Diffusion und konvektive Prozesse dringt Wasserstoff aus der umgebenden Atmosphäre in die Schmelze ein. Nach dem Erreichen der maximalen Aufschmelztiefe bewegt sich die Erstarrungsfront mit einer Geschwindigkeit von $\mathrm{v}_{s / l}^{c-S i}\left(5 \mathrm{~J} / \mathrm{cm}^{2}\right)=1.6 \mathrm{~m} / \mathrm{s}(\mathrm{s}$. Abb. 70 zurück in Richtung der Probenoberfläche. Durch die hohen Geschwindigkeiten für die Rekristallisation aus der flüssigen Phase gelingt die Rekristallisation zunächst mit der Bewegung der Erstarrungsfront. Aufgrund der höheren Löslichkeit des Wasserstoffs in der Siliziumschmelze (siehe „Si-H-Phasendiagramm“, Anhang F.2) bewegt sich der Wasserstoff mit der Grenzfläche flüssig/fest zurück in Richtung der Probenoberfläche. Nach ca. 950 ns hat die Erstarrungsfront die Probenoberfläche erreicht. Dort ist die Rekristallisation nicht vollständig und der Wasserstoff dekoriert die weiter oben beschriebenen oberflächennahen Defektstellen.

Dieses Szenario erklärt die Beobachtungen und ist mit der gezeigten Temperatursimulation verträglich. Allerdings hängt die Erklärung für die oberflächennahen Defekte von der Rekristallisationsgeschwindigkeit in der flüssigen Phase ab, die experimentell nur schwer zugänglich ist. Zur Klärung der Prozesse, die zu der gestörten Si-Oberfläche durch die Laserbestrahlung führen, könnten Experimente zum Laserhydrieren mit einem fein fokussierten homogenisierten Laserstrahl dienlich sein. Der homogenisierte Laserstrahl erlaubt eine sehr viel genauere Einstellung der Laserfluenzen. Somit wäre es möglich die Abhängigkeit der Schädigung von der eingestellten Laserfluenz detailliert zu untersuchen. Zusätzlich sind auch hier Experimente mit gekühlten Proben denkbar, um das Ausdiffundieren des Wasserstoffs während der Laserbestrahlung zu verhindern. 


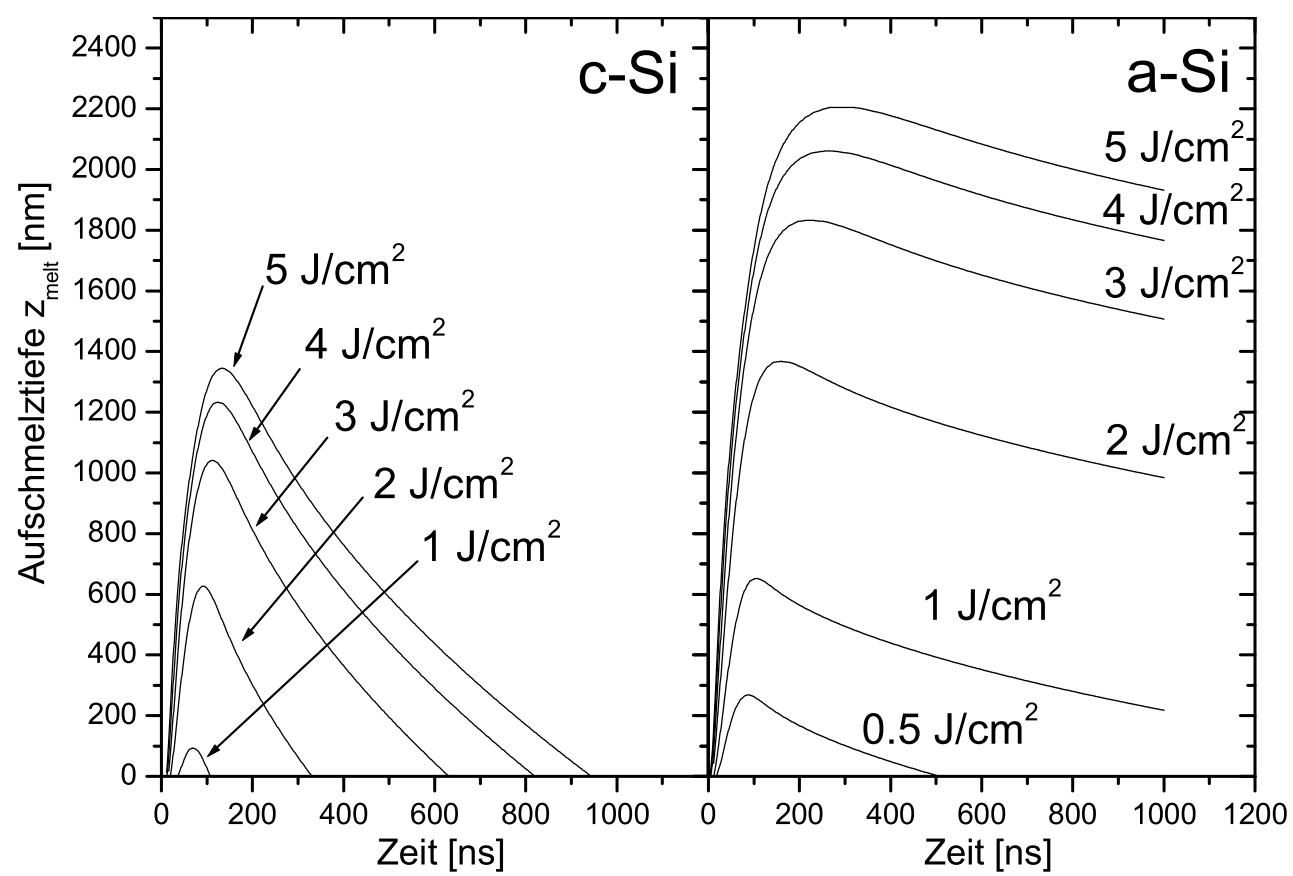

Abbildung 69: Simulation der Aufschmelztiefe für kristallines Si (rechts) und amorphes Si (links) als Funktion der Zeit

\section{Amorphes Silizium}

Bei den Untersuchungen zur Ionenstrahl-Amorphisierung von Silizium mittels $450 \mathrm{keV}$ Xe-Ionen wurde für die Erzeugung einer vollständig amorphisierten Schicht eine kritische Fluenz von $\phi_{\text {crit }}=5 \times 10^{13} \mathrm{Xe} / \mathrm{cm}^{2}$ experimentell bestimmt. Durch die Implantation von $\phi=5 \times 10^{15} \mathrm{Xe} / \mathrm{cm}^{2}$ bildete sich eine amorphe Schicht der Dicke $400 \mathrm{~nm}$ und die Rauigkeit der Probenoberfläche nahm von $\mathrm{R}_{a}($ vor $)=4(1) \mathrm{nm}$ auf den Wert $\mathrm{R}_{a}$ (nach) $=6(1) \mathrm{nm} \mathrm{zu}$. Nach dem Laserhydrieren mit $\mathrm{F}=4 \mathrm{~J} / \mathrm{cm}^{2}$, also oberhalb der Plasmaschwelle, steigt der Rauigkeitswert hingegen auf den zehnfachen Wert an.

Schon bei der kleinsten genutzten Laserfluenz von $0.5 \mathrm{~J} / \mathrm{cm}^{2}$ zeigen die RBS-ChannelingMessungen, dass die Schädigung im Bereich der Grenzschicht zwischen amorphem und kristallinem Bereich abnimmt. Die einsetzende Rekristallisation hat dabei zunächst keinen Einfluss auf das Tiefenprofil des Xenons im amorphisierten Bereich. Bei Steigerung der Laserfluenz nimmt die Schädigung immer weiter ab und es kommt zur Xe-Diffusion in Richtung der Probenoberfläche. Die Tatsache, dass sich die normierte Schädigung im Mittel absenkt, die Schädigungstiefe sich jedoch nicht ändert, scheint auf den ersten Blick der Vorstellung von der epitaktischen Rekristallisation in der Siliziumschmelze zu widersprechen. Bei der Rekristallisation, ausgehend von der kristallinen Seite, sollte es zu einem planaren Wachstum der Kristallebenen kommen. Daher sollte anschaulich die Dicke der amorphisierten Schicht immer weiter abnehmen. Die Abnahme der Dicke der amorphisierten Schicht kann z.B. bei der thermischen Rekristallisation (SPEG) von $\alpha$-Quartz sehr genau beobachtet werden [92, 124, 125]. Bei den hier gezeigten Schädigungsprofilen tritt diese Abnahme der Dicke jedoch nicht auf. Die Erklärung hierfür liegt in der ChannelingMessmethode begründet und erklärt sich wie folgt:

Durch die Inhomogenität des hier benutzten Laser-Rohstrahls kommt es in einigen Berei- 


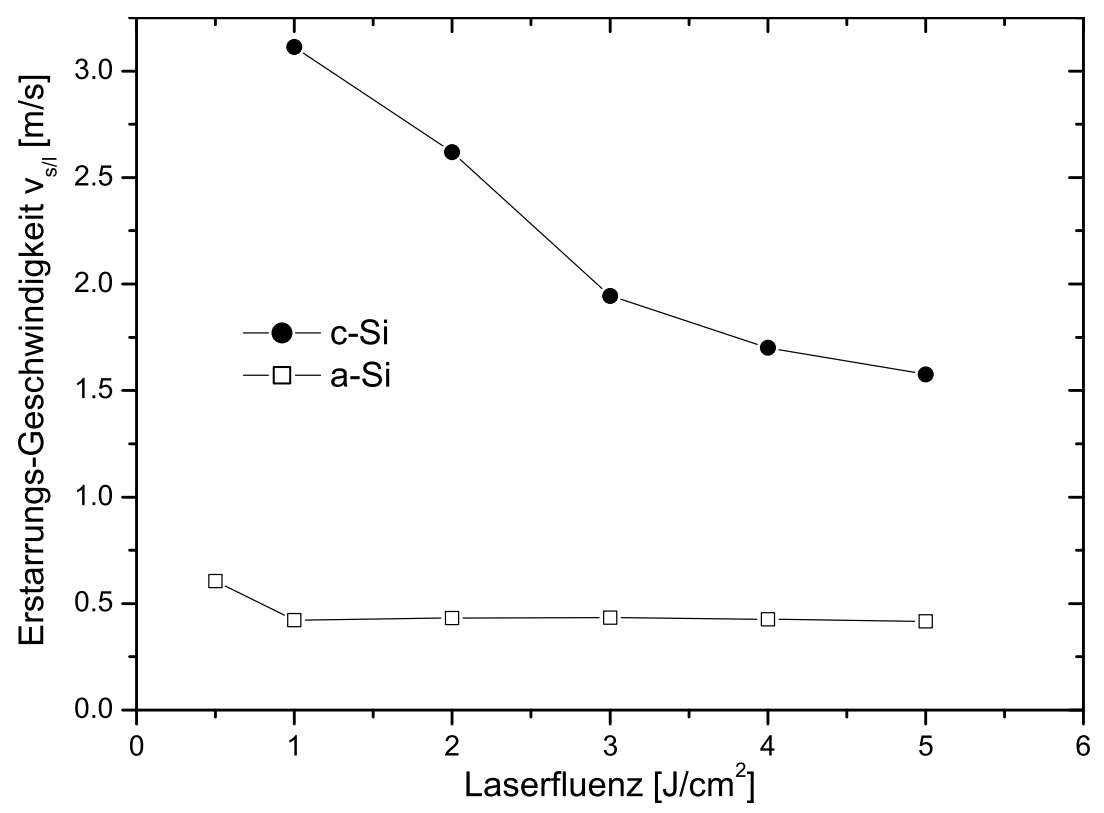

Abbildung 70: Aus der (negativen) Steigung des fallenden Zweiges der Aufschmelztiefe in Abb. 69 berechnete mittlere Erstarrungsgeschwindigkeit $v_{s / l}$

chen der bestrahlten Schicht bereits zur Rekristallisation, während die Fluenz des Lasers in anderen Bereichen zum Aufschmelzen nicht ausreicht. Wird nun eine solch inhomogen rekristallisierte Probe mittels RBS-Channeling vermessen, so ergibt sich aus dem Durchmesser des RBS-Analysestrahls von ca. $1 \mathrm{~mm}$ eine Mittelung über verschieden stark rekristallisierte Bereiche. Dies führt in den Channeling-Spektren zu einer Abnahme der mittleren Schädigung, nicht aber der maximalen Schädigungstiefe. Wichtig ist an dieser Stelle, dass mittels RBS-Channeling nicht zwischen polykristallinem Si und geschädigten Bereichen unterschieden werden kann. Nur wenn epitaktisches Kristallwachstum vorliegt zeigt sich dies in einer geringeren Rückstreurate in den Channeling-Spektren. Durch XRDMessungen konnte gezeigt werden, dass sich in diesem Übergangsbereich polykristallines Material gebildet hat. Für die durch die Laserbestrahlung entstehenden Kristallite wurde die Größe $\bar{D}=64(8) \mathrm{nm}$ ermittelt. Dieser Wert entspricht den Ergebnissen, die Toet et al. [126] für die Laser-Rekristallisation von a-Si:H-Filmen angeben. Toet et al. finden für die Rekristallisation eines $90 \mathrm{~nm}$ dicken a-Si:H-Films auf einem Glass-Substrat nach der Bestrahlung mit einem XeCl-Excimerlaser bei der Fluenz $0.3 \mathrm{~J} / \mathrm{cm}^{2}$ eine Kristallitgröße von $\bar{D}=68(2) \mathrm{nm}$.

Eine Steigerung der Laserfluenz auf $\mathrm{F}=1.5 \mathrm{~J} / \mathrm{cm}^{2}$ führt zur vollständigen epitaktischen Rekristallisation der Probe. Durch das Tiefenprofil des verbliebenen Xenons konnte zweifelsfrei belegt werden, dass es sich dabei nicht um ein einfaches Abtragen der amorphen Schicht handelt.

Die durchgeführten NRA-Messungen ergaben nur in zwei Fällen eine messbare Wasserstoffaufnahme des amorphen Siliziums. Für die Laserfluenz $\mathrm{F}=1 \mathrm{~J} / \mathrm{cm}^{2}$, die gerade noch nicht zum vollständigen Rekristallisieren ausreichte, wurde eine erhöhte Wasserstoffkonzentration bis in eine Tiefe von $40 \mathrm{~nm}$ gemessen. Ausserdem zeigte eine nur mit 1 Laserpuls der maximalen Fluenz $\mathrm{F}=5 \mathrm{~J} / \mathrm{cm}^{2}$ bestrahlte a-Si-Probe einen erhöhten Wasserstoffgehalt nahe der Probenoberfläche. 
Das Szenario zum Wasserstoffeintrag ist dabei ähnlich zu demjenigen im kristallinen Fall. Für die Bestrahlung mit der Laserfluenz $F=1 \mathrm{~J} / \mathrm{cm}^{2}$ sagt die Simulation eine maximale Aufschmelztiefe von ca. $600 \mathrm{~nm}$ voraus, d.h. die gesamte amorphe Schicht wird hier bereits aufgeschmolzen. Allerdings ist die Geschwindigkeit der Erstarrungsfront für das amorphe Silizium mit $\mathrm{v}_{s / l}^{a-S i}\left(1 \mathrm{~J} / \mathrm{cm}^{2}\right)=0.4 \mathrm{~m} / \mathrm{s}$ wesentlich geringer als im kristallinen Fall $(3.1 \mathrm{~m} / \mathrm{s}$, bei $1 \mathrm{~J} / \mathrm{cm}^{2}$ ). Durch die langsame Bewegung der Grenzfläche zwischen fester Phase und Schmelze gelingt die Diffusion des Wasserstoffs in die Schmelze wesentlich effektiver und führt so zu einem Transport des Wasserstoffs mit der Schmelze zur Probenoberfläche. Auf diese Weise kann erklärt werden, weshalb es auch bei der Bestrahlung mit hohen Laserfluenzen im Falle des amorphen Siliziums nicht gelang, Wasserstoff in die Probe einzubringen.

Die mit der Laserfluenz $\mathrm{F}=1 \mathrm{~J} / \mathrm{cm}^{2}$ bestrahlte Probe zeigte zusätzlich zum erhöhten Wasserstoffgehalt auch einen ausgedehnten geschädigten Bereich. Es treten also wieder Defekte und Wasserstoff gemeinsam auf. Da Defekte als Senken für die Wasserstoffatome wirken, hat auch der Grad der Rekristallisation Einfluss auf den Wasserstoffgehalt. Daher ist bei vollständig rekristallisierten Proben ein geringerer Wasserstoffgehalt zu erwarten, wie auch experimentell bestätigt wurde.

Für weiterführende Untersuchungen der Abhängigkeit der H-Aufnahme vom Kristallisationsgrad der Proben erscheint die RBS-Methode aus den oben genannten Gründen ungeeignet. Für a-Si-Proben, die im Laserfluenzbereich $0.75 \mathrm{~J} / \mathrm{cm}^{2}<\mathrm{F}<1.5 \mathrm{~J} / \mathrm{cm}^{2}$ bestrahlt wurden, in dem die RBS-Channeling-Messungen noch keine vollständige epitaktische Rekristallisation anzeigten, wäre eine TEM-Untersuchung sinnvoll. Damit könnte das polykristalline Material klar von den Defekten unterschieden werden. Ebenso würden optische Methoden wie Raman-Spektroskopie oder Photolumineszens-Spektroskopie eine Untersuchung des polykristallinen Materials erlauben.

Für die Implantation von $15 \mathrm{keV} \mathrm{H}^{+}$-Ionen in Silizium wurde die kritische Fluenz $\phi_{\text {crit }}=9 \times 10^{16} \mathrm{H} / \mathrm{cm}^{2}$ experimentell bestimmt. Bei dieser Ionenfluenz bildet sich in einer Tiefe von $180 \mathrm{~nm}$ eine amorphisierte Schicht der Dicke $80 \mathrm{~nm}$. Es konnte mittels NRA-Messungen gezeigt werden, dass die Position des Implantationsprofils mit dem geschädigten Bereich übereinstimmt. Während das Monte-Carlo-Programm SRIM2000 die Lage des Implantationsmaximums überschätzt, stimmt die Position des Schwerpunktes der Schädigung $\bar{z}_{\text {dam }}$ mit dem experimentellen Ergebnis überein.

Der Vergleich der Wasserstofftiefenprofile vor und nach dem Laserhydrieren der H-implantierten Proben zeigt einen starken Wasserstoffverlust durch die Laserbehandlung. Da gleichzeitig die Oberflächenrauigkeit der Proben schon bei kleiner Laserfluenz stark zunimmt, muss davon ausgegangen werden, dass die laserinduzierte Temperaturerhöhung zu einem Aufplatzen der Wasserstoff-Agglomerate führt. In Übereinstimmung mit dieser Hypothese zeigen die laserbehandelten H-implantierten Proben eine starke Schädigung, die von der Probenoberfläche bis in die Tiefe des Implantationsschadens reichte. Es wurden keine Anzeichen für eine einsetzende Rekristallisation des geschädigten Bereichs beobachtet.

Für zukünftige Untersuchungen an H-implantierten Siliziumproben erscheint daher der Laserfluenzbereich $\mathrm{F}<1 \mathrm{~J} / \mathrm{cm}^{2}$ sinnvoll, um ein Aufplatzen der Probenoberfläche zu verhindern. Mit Hilfe von Siliziumproben, die mit unterschiedlichen Energien von H-Ionen implantiert werden, könnte außerdem der simulierte Temperaturverlauf bei der Laserbestrah- 
lung sehr gut experimentell überprüft werden. Wegen des geringen Bremsvermögens der Wasserstoffionen werden schon mit geringer Ionenenergie große Reichweiten der H-Ionen im Silizium erzielt. Dadurch lassen sich sehr einfach Wasserstoff-Implantationsprofile in Silizium als Markerschicht, auch in großer Tiefe, verwenden. Die Nutzung von Wasserstoff als Marker hat gegenüber anderen Ionen den Vorteil, dass die Strahlenschäden bei der Implantation äußerst gering sind, sofern die Ionenfluenz $10^{16} \mathrm{H} / \mathrm{cm}^{2}$ nicht überschreitet. NRA-Messungen vor und und nach der Laserbestrahlung erlaubten dann Rückschlüsse auf die Temperatur am Ort des Implantationsprofils während der Laserbestrahlung.

\section{Schlussbetrachtung}

Bei der vorliegenden Arbeit handelt es sich um die erste Anwendung des neuaufgebauten MaRPel-Beschleuniger-Labors. Es wurde gezeigt, dass alle wesentlichen Komponenten der Strahlerzeugung und Strahlführung bis hin zum Messplatz für Low-Level Kernreaktionsanalyse voll funktionstüchtig sind. Mit dem MaRPel-Labor eröffnen sich eine ganze Reihe neuer experimenteller Möglichkeiten, deren wichtigstes Beispiel die hochgenaue Wasserstoffanalyse ist. Ohne die experimentellen Voraussetzungen, Wasserstoffkonzentrationen von weniger als 0.5 at\% in Silizium nachzuweisen, wäre eine Untersuchung der Vorgänge beim Laserhydrieren nicht möglich gewesen.

Für die in der Einleitung geäußerte Idee, das Laserhydrieren als Prozess zur Erzeugung von polykristallinem Material mit großer Kristallitgröße und hohem Wasserstoffanteil $\mathrm{zu}$ verwenden, sind die gefundenen Ergebnisse leider unzureichend. Bei der LaserRekristallisation von a-Si:H zur Anwendung als TFT-Material werden mittlerweile typische Werte für die Kristallitgröße von einigen hundert Nanometern erreicht [127]. Der geforderte Wasserstoffgehalt von ca. 16 at\% wird durch das Laserhydrieren ebenfalls nicht hergestellt. 


\section{Literaturverzeichnis}

[1] F. Hinterberger, Physik der Teilchenbeschleuniger und Ionenoptik (Springer, Berlin Heidelberg New York, 1997).

[2] M. Uhrmacher, K. Pampus, F. J. Bergmeister, D. Purschke, and K. P. Lieb, Nucl. Instr. and Methods B 9, 234 (1985).

[3] P. Schaaf, Progr. Mat. Sci. 47(1), 1 (2002).

[4] J. B. Marion and F. C. Young, Nuclear Reaction Analysis, Graphs and Tables (North Holland, Amsterdam, 1968), p. 145.

[5] J. C. Overley, P. D. Parker, and D. A. Bromley, Nucl. Instr. and Methods 68, 61 (1969).

[6] R. Middleton and C. T. Adams, Nucl. Instr. and Methods 118, 329 (1973).

[7] H. V. Smith and H. T. Richards, Nucl. Instr. and Methods 125, 497 (1975).

[8] R. Middleton, Nucl. Instr. and Methods 144, 373 (1977).

[9] G. T. Caskey, R. A. Douglas, H. T. Richards, and H. V. Smith, Nucl. Instr. and Methods 157, 1 (1978).

[10] R. J. Girnius, L. W. Anderson, and E. Staab, Nucl. Instr. and Methods 143, 505 (1977).

[11] A. S. Schlachter, P. J. Bjorkholm, D. H. Loyd, L. W. Anderson, and W. Haeberli, Phys. Rev. 177(1), 184 (1969).

[12] R. J. Girnius and L. W. Anderson, Nucl. Instr. and Methods 137, 373 (1976).

[13] http://www.pelletron.com/charging.htm (National Electrostatics Corp., Middleton (USA), 2002).

[14] G. W. Roth and W. G. Weitkamp, Nucl. Instr. and Methods 115, 501 (1974).

[15] G. Amsel, M. Menu, J. Moulin, and J. Salomon, Nucl. Instr. and Methods B45, 296 (1990).

[16] J. A. Ferry, Nucl. Instr. and Methods A 328, 28 (1993).

[17] Terminal Potential Stabilizer Controller Mod. TPS-5.5 Instruction Manual (Man. No. 2HA047770M) (National Electrostatics Corp., Middleton (USA), 1989).

[18] W. A. Lanford, H. P. Trautvetter, J. F. Ziegler, and J. Keller, Appl. Phys. Letters 28, 566 (1976).

[19] F. Xiong, F. Rauch, C. Shi, Z. Zhou, R. P. Livi, and T. A. Tombrello, Nucl. Instr. and Methods B27, 432 (1987). 
[20] G. Deconninck and B. V. Oystaeyen, Nucl. Instr. and Methods 218, 165 (1983).

[21] G. Amsel and B. Maurel, Nucl. Instr. and Methods 218, 183 (1983).

[22] W. H. Bragg and R. Kleeman, Philos. Mag. 10, 318 (1905).

[23] H. H. Andersen and J. F. Ziegler, Hydrogen Stopping Powers and Ranges in All Elements (Pergamon Press, New York, 1977).

[24] G. Schatz and A. Weidinger, Nukleare Festkörperphysik (B. G. Teubner, Stuttgart, 1992).

[25] A. Schardt, W. A. Fowler, and C. C. Lauritsen, Phys. Rev. 86, 527 (1952).

[26] T. Osipowicz, K. P. Lieb, and S. Brüssermann, Nucl. Instr. and Methods B 18, 232 (1987).

[27] P. Vavilov, Sov. Phys. JETP 5, 749 (1957).

[28] E. Bonderup and P. Hvelplund, Phys. Rev. A 4, 562 (1971).

[29] H. Bichsel and R. Saxon, Phys. Rev. A 11, 1286 (1975).

[30] N. Bohr, Mat. Fys. Medd. Dan. Vidensk. Selsk. 18(8),24, 19 (1948).

[31] J. Lindhard and M. Scharff, Mat. Fys. Medd. Dan. Vidensk. Selsk. 27, 15 (1953).

[32] M. Zinke-Allmang, Dissertation, MPI für Kernphysik, Heidelberg, 1985.

[33] M. Zinke-Allmang, S. Kalbitzer, and M. Weiser, Z. Phys A 320, 697 (1985).

[34] M. Zinke-Allmang, V. Kössler, and S. Kalbitzer, Nucl. Instr. and Methods B 15, 563 (1986).

[35] M. Zinke-Allmang, S. Kalbitzer, and M. Weiser, Z. Phys A 325, 183 (1986).

[36] Angewandte Kernphysik, edited by W. T. Hering (Teubner Studienbücher, Stuttgart, Leipzig, 1999).

[37] P. Oberschachtsiek, M. Weiser, and S. Kalbitzer, Nucl. Instr. and Methods B56/57, 1010 (1991).

[38] T. W. Aitken, A. E. Groome, T. Joy, and W. J. Myring, Nucl. Instr. and Methods A 244, 183 (1986).

[39] B. Hartmann, S. Kalbitzer, and C. Klatt, Nucl. Instr. and Methods B124, 490 (1997).

[40] O. Vollmer, Nucl. Instr. and Methods 121, 373 (1974).

[41] B. Efken, D. Hahn, and G. Wüstefeld, Nucl. Instr. and Methods 129, 219 (1975). 
[42] Taschenbuch der Physik, edited by H. Stöcker (Verlag Harri Deutsch, Frankfurt/Main, 1998).

[43] HVI-369 Manual 3 (1980).

[44] R. O. Bondelid and C. A. Kennedy, NRL Report, 1958.

[45] S. L. Andersen, H. Bö, T. Holtebekk, . Lönsjö, and R. Tangen, Nucl. Phys. 9, 509 (1959).

[46] M. Schwickert, F. Harbsmeier, H. Schebela, M. Uhrmacher, E. Carpene, P. Schaaf, and K. P. Lieb, Surf. Coat. Technol. 151/152, 222 (2002).

[47] A. Schardt, W. Fowler, and C. Lauritsen, Phys. Rev. 86, 527 (1952).

[48] S. Bashkin and R. R. Carlson, Phys. Rev. 106, 261 (1957).

[49] G. P. Plain, R. G. Herb, C. M. Hudson, and R. E. Warren, Phys. Rev. 57, 187 (1940).

[50] G. Amsel, E. d'Artemare, and E. Girard, Nucl. Instr. and Methods 205, 5 (1983).

[51] P. M. Endt and C. V. der Leun, Nucl. Phys. A 105, 140 (1967).

[52] H. Becker, Diplomarbeit, Max-Planck-Institut für Kernphysik Heidelberg, 1991.

[53] W. K. Chu, J. W. Mayer, and M.-A. Nicolet, Backscattering Spectrometry (Academic Press, Orlando, 1978).

[54] J. A. Leavitt, J. L. C. McIntyre, and M. R. Weller, in Handbook of Modern Ion Beam Materials Analysis, edited by J. R. Tesmer, M. Nastasi, C. J. Maggiore, J. C. Barbour, and J. W. Mayer (Materials Research Society, Pittsburgh, 1995), p. 37.

[55] E. Rutherford, Phil. Mag. 21, 669 (1911).

[56] L. R. Doolittle, Nucl. Instr. and Methods B 9, 344 (1985).

[57] J. Conrad, Dissertation, Universität Göttingen, 1996.

[58] J. Stark, Z. Phys. 13, 973 (1912).

[59] M. T. Robinson and O. S. Oen, Phys. Rev. 132, 2385 (1963).

[60] R. S. Walker and D. A. Thompson, Nucl. Instr. and Methods 135, 489 (1976).

[61] F. Harbsmeier, Diplomarbeit, Universität Göttingen, 1996.

[62] DIN4768, Rauigkeitsmessgrößen.

[63] C. Illgner, P. Schaaf, K. P. Lieb, R. Queitsch, and J. Barnikel, J. Appl. Phys. 83, 2907 (1998).

[64] C. Illgner, Dissertation, Universität Göttingen, 1997. 
[65] F. Landry, Dissertation, Universität Göttingen, 1999.

[66] F. Landry, K. P. Lieb, and P. Schaaf, Nucl. Instr. and Methods B 161-163, 609 (2000).

[67] H. R. Christen, Chemie (Diesterweg, Frankfurt a. M., 1974).

[68] T. Sameshima, M. Sekiya, M. Hara, N. Sano, and A. Kohno, J. Appl. Phys. 76 (11), 7377 (1994).

[69] M. Stutzman, W. B. Jacson, and C. C. Tai, Phys. Rev. B 34, 63 (1986).

[70] J. F. Ziegler, J. P. Biersack, and U. Littmark, The Stopping and range of Ions in Solids (Pergamon Press, New York, 1999), Vol. 1.

[71] W. Bolse, Mat. Sci. Eng. R 12, 53 (1994).

[72] M. W. Guinan and J. H. Kinney, J. Nucl. Mat. 103/104, 1319 (1981).

[73] W. King and R. Benedek, J. Nucl. Mat. 117, 257 (1980).

[74] J. F. Gibbons, Proc. IEEE 60, 1062 (1972).

[75] J. R. Dennis and E. B. Hale, J. Appl. Phys. 49, 1119 (1978).

[76] Laser-Beam-Interactions with Materials, edited by M. von Allmen and A. Blatter (Springer Series in Mat. Sci., Berlin Heidelberg New York, 1995).

[77] CRC Handbook of Chemistry and Physics, edited by R. C. Weast and M. J. Astle (CRC Press, Boca Raton, 1982).

[78] D. Bäuerle, Laser Processing and Chemistry (Springer-Verlag, Berlin Heidelberg New York, 2000).

[79] D. Auston, J. Golovshenko, A. Simons, R. Slusher, R. Smith, C. Murko, and T. Venkatesan, Appl. Phys. Letters 34, 777 (1979).

[80] P. Baeri and S. U. C. in J. M. Poate and J. W. Mayer (eds. ), Laser Annealing in Semiconductors (Academic Press, New York, 1982).

[81] E. Carpene, Dissertation, Universität Göttingen, 2002.

[82] S. Fähler and H. U. Krebs, Appl. Surf. Sci. 96, 61 (1996).

[83] Y. S. Touloukian and E. H. Buyco, in Thermophysical Properties of Matter (vol.4), edited by Y. S. Touloukian and C. Y. Ho (IFI / Plenum, New York, 1983).

[84] Y. S. Touloukian, R. W. Powell, C. Y. Ho, and P. G. Klemens, in Thermal Conductivity, metallic elements and alloys (vol.1), edited by Y. S. Touloukian and C. Y. Ho (IFI / Plenum, New York, 1970). 
[85] Y. S. Touloukian, R. W. Powell, C. Y. Ho, and M. C. N. in, in Thermophysical Properties of Matter (vol.10), edited by Y. S. Touloukian and C. Y. Ho (IFI / Plenum, New York, 1973).

[86] L. Csepregi, E. F. Kennedy, J. W. Mayer, and T. W. Sigmon, J. Appl. Phys. 49, 3906 (1978).

[87] G. M. Weyl, in Laser-induced Plasmas and Applications, edited by L. J. Radziemski and D. A. Cremers (Marcel Dekker, New York, 1992).

[88] D. I. Rosen, J. Mitteldorf, G. Kothandaramn, A. N. Piri, and E. R. Pugh, J. Appl. Phys. 53, 3190 (1982).

[89] D. I. Rosen, D. E. Hastings, and G. M. Weyl, J. Appl. Phys. 53, 5882 (1982).

[90] Y. P. Raizer, Sov. Phys. JETP 31, 1148 (1970).

[91] G. L. Olson and J. A. Roth, Mat. Sci. Rep. 3, 1 (1988).

[92] F. Roccaforte, W. Bolse, and K. P. Lieb, Appl. Phys. Letters 73, 1349 (1998).

[93] F. Roccaforte, W. Bolse, and K. P. Lieb, J. Appl. Phys. 89(7), 3611 (2001).

[94] J. Narayan, C. W. White, M. J. Aziz, B. Stritzker, and A. Walthuis, J. Appl. Phys.

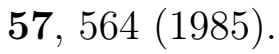

[95] A. G. Cullis, H. Webber, N. G. Chew, J. M. Poate, and P. Baeri, Phys. Rev. Lett. 49, 219 (1982).

[96] G. Foti and E. Rimini in J. M. Poate and J. W. Mayer (eds. ), Laser Annealing in Semiconductors (Academic Press, New York, 1982).

[97] G. Foti, E. Bertolotti, and G. Vitali, Phys. Lett. A 65, 430 (1978).

[98] P. Lengsfeld, N. H. Nickel, and W. Fuhs, Appl. Phys. Letters 76 (13), 1680 (2000).

[99] Y. Kamiura, M. Yoneta, and F. Hashimoto, Appl. Phys. Letters 59 (24), 3165 (1991).

[100] A. van Wieringen and N. Warmoltz, Physica 22, 849 (1956).

[101] C. H. Seager and R. A. Anderson, Sol. St. Comm. 76 (3), 285 (1990).

[102] S. M. Myers, D. M. Follstaedt, H. J. Stein, and W. R. Wampler, Phys. Rev. B 47(20), 13380 (1993).

[103] S. Wagner, Diplomarbeit, Universität Göttingen, 2000.

[104] S. Habenicht, Dissertation, Universität Göttingen, 1999.

[105] S. Habenicht, W. Bolse, and K. P. Lieb, Rev. of Scient. Instr. 69(5), 2120 (1998). 
[106] H. Hofsäss, H. Binder, T. Klumpp, and E. Recknagel, Diam. Relat. Mater. 3, 137 (1994).

[107] M. Han, Dissertation, Universität Göttingen, 2001.

[108] E. Carpene, P. Schaaf, M. Han, K. P. Lieb, and M. Shinn, Appl. Surf. Sci. 7364, 1 (2001).

[109] R. Sailer and G. McCarthy, North Dakota State University, ICDD Grant-in-Aid (1993).

[110] A. Crane et al., J. Less-Common Met. 25, 225 (1971).

[111] ID07-0370, Powder Diffraction File Database PDF2 (International Centre for Diffraction, Newton Square, 1997), pp. 07-0370.

[112] W. E. Spear and P. G. LeComber, Solid State Commun. 17, 1193 (1975).

[113] Natl. Bur. Stand. (US), Monogr. 25 13, 53 (1976).

[114] R. Job, A. G. Ulyashin, W. R. Fahrner, A. I. Ivanov, and L. Palmetshofer, Appl. Phys. A 72, 325 (2001).

[115] T. Höchbauer, A. Misra, M. Nastasi, and J. W. Mayer, J. Appl. Phys. 89(11), 5980 (2001).

[116] X. Duo, W. Liu, S. Xing, M. Zhang, X. Fu, C. Lin, P. Hu, S. X. Wang, and L. M. Wang, J. Phys. D 34, 5 (2000).

[117] M. Bruel, B. Aspar, B. Charlet, C. Maleville, T. Poumeyrol, A. Soubie, A. J. Auberton-Herve, J. M. Lamure, T. Borge, F. Metral, and S. Trucchi, Proceedings IEEE Tucson 178 (1995).

[118] M. Bruel, Nucl. Instr. and Methods B108, 313 (1996).

[119] M. Bruel, MRS Bulletin 12, 35 (1998).

[120] K. Suzuki, M. Takahashi, and M. Saitoh, Appl. Phys. A 69 [Suppl.], 263 (1999).

[121] P. L. Liu, R. Yen, N. Bloembergen, and R. T. Hodgson, Appl. Phys. Letters 34(12), 864 (1979).

[122] F. Spaepen and D. Turnbull in J. M. Poate and J. W. Mayer (eds.), Laser Annealing in Semiconductors (Academic Press, New York, 1982), pp. 15-41.

[123] N. Bloembergen in S. D. Ferris, H. J. Leamy, and J. M. Poate (eds.), Laser-Solid Interactions and Laser Processing (Am. Inst. Phys., New York, 1979), p. 1.

[124] F. Roccaforte, Dissertation, Universität Göttingen, 1999.

[125] F. Roccaforte, W. Bolse, and K. P. Lieb, Nucl. Instr. and Methods B 148, 692 (1999). 
[126] D. Toet, P. M. Smith, T. W. Sigmon, T. T. anc C. C. Tsai, W. R. Harsharger, and M. O. Thompson, J. Appl. Phys. 85(11), 7914 (1999).

[127] M. Lee, S. Moon, M. Hatano, and C. P. Grigoropoulos, Appl. Phys. A(73), 317 (2001).

[128] http://www.trolltech.com (Trolltech AS, Oslo (Norway), 2002).

[129] http://www.gnu.org/copyleft/gpl.html (Free Software Foundation, Boston (USA), 2002).

[130] H. Okamoto, Journal of Phase Equilibria 13(4), (1992).

[131] T. K. Kostina, B. A. Baum, and K. T. Kuroschkin, Akad. Nauk SSSR. Izv. Neorg. Mater. 6, 117 (1970). 


\section{Abbildungsverzeichnis}

1 Wesentliche Komponenten des MaRPel-Beschleunigerlabors . . . . . . . . . 5

$2 \quad$ Ehemaliges Halbleiter-Labor des MPI-K Heidelberg . . . . . . . . . . . . . 7

3 $\quad$ Erster He-Strahl . . . . . . . . . . . . . . . . . . . . . . . . . . . . . . . . . 9

$4 \quad$ Prinzipskizze der SNICS-Ionenquelle $\ldots \ldots \ldots$. . . . . . . . . . . . . . . 10

$5 \quad$ Skizze der Alphatross-Ionenquelle . . . . . . . . . . . . . . . . . . . . . . . 11

$6 \quad$ Prinzipskizze des Ladungssystems $\ldots \ldots$. . . . . . . . . . . . . . . 12

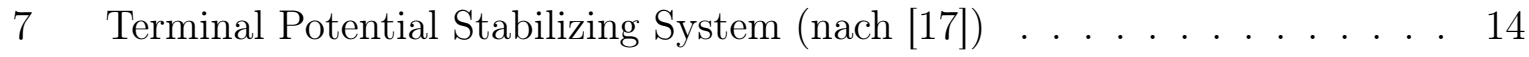

$8 \quad$ Prinzip der resonanten Kernreaktionsanalyse . . . . . . . . . . . . . . . . . 16

$9 \quad$ Reaktionsschema, Energien in $\mathrm{MeV}$. . . . . . . . . . . . . . . . . . . . . . 17

$10 \quad$ NRA-Ortsauflösung mit Protonen- und ${ }^{15} \mathrm{~N}-\mathrm{Strahl}$. . . . . . . . . . . . . . 18

$11 \quad 0^{\circ}$-Grad-Strahlrohr . . . . . . . . . . . . . . . . . . . . . . . . . 19

12 Skizze der Zweifach-Viersektorenblende . . . . . . . . . . . . . . . . . . . . 20

13 Schema der Antikoinzidenz-Schaltung . . . . . . . . . . . . . . . . . . . . . 21

14 Spektren zum Gammastrahlungs-Untergrund . . . . . . . . . . . . . . . . . 21

15 Terminalripple, gemessen mit einem Speicheroszillographen . . . . . . . . . 23

$16 \quad$ NRA-Spektrum einer dicken Al-Probe . . . . . . . . . . . . . . . . . . . . . 25

17 Resonanzkurven der ${ }^{15} N(p, \alpha \gamma){ }^{12} C$-Kernresonanz . . . . . . . . . . . . . . 27

18 Magnetfeld des $90^{\circ}$-Magneten als Funktion der Ionenenergie . . . . . . . . 28

19 Strahlverlauf in Amsel-Deflektoren und 90-Magnet . . . . . . . . . . . . . 29

$20 \quad$ Experimenteller Verstärkungsfaktor $G_{0}(\exp$.) für Protonen . . . . . . . . . 31

21 Experimenteller Verstärkungsfaktor, bestimmt mit $10 \mathrm{~nm}$ Al-Schicht . . . . 31

22 Experimenteller Verstärkungsfaktor $G_{0}(\exp$.$) für { }^{15} \mathrm{~N}-$ Ionen . . . . . . . . . 32

23 KNRA-Hauptfenster . . . . . . . . . . . . . . . . . . . . 33

24 KNRA-Messfenster . . . . . . . . . . . . . . . . . . . . . . . . . . . 34

$25 \quad$ KNRA-Analysefenster und Amselcontrol . . . . . . . . . . . . . . . . . . . 34

26 Reproduzierbarkeit von NRA-Spektren . . . . . . . . . . . . . . . . . . . 36

27 Prinzipskizze RBS . . . . . . . . . . . . . . . . . . . . . . . . . . 37

28 RBS-Beispielspektrum . . . . . . . . . . . . . . . . . . . . . . . . . . 38

29 Beispielspektren für RBS-C . . . . . . . . . . . . . . . . . . 40 
$30 \quad$ Prinzip der Rauigkeitsmessungen mittels Oberflächenprofilometrie . . . . . 41

$31 \quad$ SRIM2000-Simulation zur Implantation von Xe- und H-Ionen . . . . . . . 44

$32 \quad$ SRIM2000-Simulationen zur Implantat- und Schadensverteilung . . . . . . 45

33 Simulation der Aufschmelztiefe für Titan . . . . . . . . . . . . . . . . . . . 49

34 Simulation der Oberflächentemperatur für c-Si . . . . . . . . . . . . . 50

35 Simulation der Oberflächentemperatur für a-Si . . . . . . . . . . . . . 50

36 Diffusionskonstante von $\mathrm{H}$ in $\mathrm{Si}$. . . . . . . . . . . . . . . . . . . 54

37 Strahlengang beim Laserhydrieren . . . . . . . . . . . . . . . . 56

$38 \quad$ Skizze zum mäanderförmigen Überstreichen der Probe. . . . . . . . . . . . 57

39 H-Tiefenprofile von Laserhydriertem Titan bei 16, 64 und 256 Laserpulsen

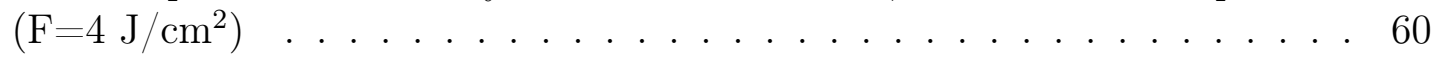

$40 \quad$ H-Gehalt von laserhydriertem Titan als Funktion der Laserpulszahl . . . . 61

$41 \quad$ NRA-Messungen an laserhydriertem Ti bei konstanter Energie . . . . . . . 62

$42 \quad$ XRD-Spektrum von laserhydriertem Titan . . . . . . . . . . . . . . . . 63

$43 \quad$ Vergrößerung des XRD-Spektrums von laserhydriertem Titan. . . . . . . . 63

44 GI-XRD-Spektrum von laserhydriertem Ti $\left(1\right.$ bar, 16 Pulse, $\left.4 \mathrm{~J} / \mathrm{cm}^{2}\right)$. . . 65

45 Wasserstofftiefenprofile von laserhydriertem c-Si . . . . . . . . . . . . 66

$46 \quad$ Höhenprofile der Oberfläche von laserhydrierten c-Si-Proben . . . . . . . . 67

47 Oberflächenrauigkeit von laserhydrierten c-Si-Proben in Abhängigkeit von der Laserfluenz $\ldots \ldots \ldots \ldots \ldots \ldots \ldots$. . . . . . . . . . . . 67

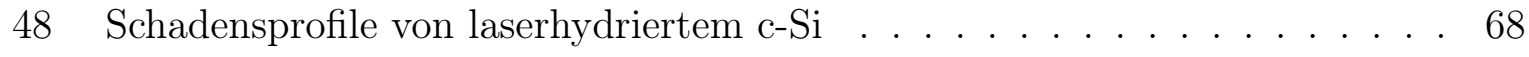

$49 \quad$ Relation zwischen Schädigung und Wasserstoffgehalt $\ldots . . . . . .69$

50 Schadensprofile von Xe-implantiertem Silizium _ . . . . . . . . . . . 71

51 Oberflächenrauigkeit von Xe-amorphisierten Si-Proben die mit 16 Laserpulsen bei 2 bar laserhydriert wurden. $\ldots \ldots \ldots \ldots . \ldots 72$

52 Schädigungs- und Xe-Verteilung einer Xe-amorphisierten Si-Probe nach Laserhydrieren mit 16 Pulsen mit $\mathrm{F}=0.5 \mathrm{~J} / \mathrm{cm}^{2} \ldots \ldots \ldots \ldots . \ldots 73$

53 Schädigungs- und Xe-Verteilung einer Xe-amorphisierten Si-Probe nach Laserhydrieren mit 16 Pulsen mit $\mathrm{F}=1 \mathrm{~J} / \mathrm{cm}^{2} \ldots \ldots \ldots \ldots \ldots . \ldots \ldots$

54 Schädigungs- und Xe-Verteilung einer Xe-amorphisierten Si-Probe nach Laserhydrieren mit 16 Pulsen mit $\mathrm{F}=1.5 \mathrm{~J} / \mathrm{cm}^{2} \ldots \ldots \ldots \ldots \ldots$

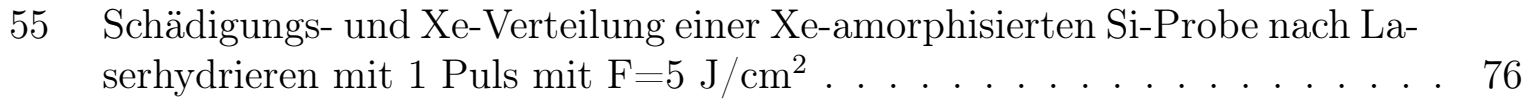


56 GI-XRD-Spektren von laserhydrierten Xe-amorphisierten Si-Proben . . . . 77

57 GI-XRD-Spektren von Xe-amorphisierten Si-Proben, z. T. laserhydriert • 77

58 H-Tiefenverteilung nach Laserydrieren xon Xe-amorphisiertem Si mit 16 Pulsen mit $\mathrm{F}=0.5-1.5 \mathrm{~J} / \mathrm{cm}^{2} \ldots \ldots \ldots \ldots \ldots$. . . . . . . . . . . . 78

59 H-Tiefenverteilung nach Laserydrieren xon Xe-amorphisiertem Si mit 16 Pulsen mit $\mathrm{F}=3-5 \mathrm{~J} / \mathrm{cm}^{2} \ldots \ldots \ldots \ldots . \ldots . \ldots . \ldots 79$

60 Schädigung, Xe-Gehalt und H-Tiefenverteilung nach Laserydrieren xon Xeamorphisiertem Si mit 16 Pulsen mit $\mathrm{F}=0.5-5 \mathrm{~J} / \mathrm{cm}^{2}$. . . . . . . . . . . . 80

$61 \quad$ Schadensprofile von H-implantiertem Silizium . . . . . . . . . . . . . . 81

62 Vergleich von Wasserstoff- und Schadensprofil bei $\phi=1.5 \times 10^{17} \mathrm{H} / \mathrm{cm}^{2}$. . . 82

63 Messung und SRIM2000-Simulation für Wasserstoff- und Schadensprofil . . 83

64 Einfluss des NRA-Analysestrahls auf die Kristallinität . . . . . . . . . . . . 85

65 Srim2000-Simulation für $6.4 \mathrm{MeV}^{15} \mathrm{~N}-$ Ionen in $\mathrm{Si}$. . . . . . . . . . . . . . 85

66 Oberfächenrauigkeit von H-implantiertem Silizium . . . . . . . . . . . . . 87

67 Foto einer laserhydrierten Probenoberfläche . . . . . . . . . . . . . . . 87

68 Wasserstoff- und Schadensprofil vor und nach Laserhydrieren $\left(\mathrm{F}=0.5 \mathrm{~J} / \mathrm{cm}^{2}\right) 88$

69 Simulation der Aufschmelztiefe von a-Si und c-Si . . . . . . . . . . . . . . . . 93

$70 \quad$ Simulation der Erstarrungsgeschwindigkeit von a-Si und c-Si . . . . . . . . 94

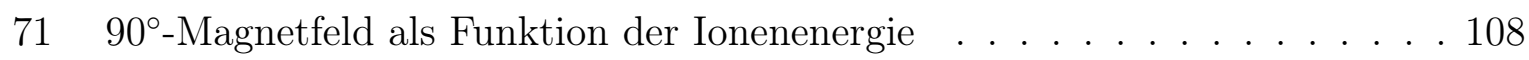

72 Geometrische Faktoren zur Berechnnung des Verstärkungsfaktors … . 109

$73 \quad$ Simulation des Verstärkungsfaktors $\left.\mathrm{G}\left(\mathrm{U}, \mathrm{V}_{0}\right)\right] \ldots$. . . . . . . . . . . . . . . 112

74 Strahlauslenkung d als Funktion der Deflektor-Spannung U . . . . . . . . . 113

75 Simulierter Verstärkungsfaktor G(E) und Näherung . . . . . . . . . . . . . 114

76 Prinzip von Realtime Linux . . . . . . . . . . . . . . . . . . 119

77 Halbleiter-Labor des MPI-K Heidelberg vor dem Abbau . . . . . . . . . . . 120

78 Halbleiter-Labor des MPI-K Heidelberg demontiert . . . . . . . . . . . . . 120

79 Transporthalterung der Beschleunigersäule . . . . . . . . . . . . . . . . 121

$80 \quad$ Verladen des Beschleuniger-Tanks mit dem Autokran . . . . . . . . . . . . 121

81 Absetzen des Beschleunigertanks in die neue Halle . . . . . . . . . . . . . . 122

82 Justieren der Ionenoptik . . . . . . . . . . . . . . . . . . . . . . . 122

83 Nullgrad-Strahlrohr während dem Neuaufbau . . . . . . . . . . . . . . . 123

84 Nullgrad-Strahlrohr mit Low-Level Messplatz . . . . . . . . . . . . . . . . 123

85 Ti-H-Phasendiagramm . . . . . . . . . . . . . . . . . . . 124

$86 \quad$ Si-H-,,Phasendiagramm ${ }^{6} \ldots \ldots \ldots \ldots \ldots \ldots$ 


\section{A Relativistische Rechnung zum $90^{\circ}$-Magneten}

Da insbesondere für leichte Ionen (Protonen) und hohe Ionenenergien relativistische Effekte beachtet werden müssen, wird die Berechnung des Magnetfeldes B zur Ablenkung von Ionen der Ruhemasse $\mathrm{m}_{0}$ und der Energie $\mathrm{E}$ um $90^{\circ}$ relativistisch durchgeführt. Aus der Formel für die relativistische kinetische Energie

$$
E=E_{k i n}=m_{0} c^{2}(\gamma-1)
$$

folgt mit der Ruheenergie $E_{0}=m_{0} c^{2}$ unter Zuhilfename von

$$
\gamma=\frac{1}{\sqrt{1-\frac{v^{2}}{c^{2}}}}
$$

für die Ionengeschwindigkeit v unmittelbar:

$$
v^{2}=c^{2} \cdot\left(1-\left(\frac{E_{0}}{E+E_{0}}\right)^{2}\right)
$$

Für die Ablenkung von Ionen der Ladung q in einem Magneten mit dem Krümmungsradius $\mathrm{R}$ und dem Magnetfeld B gilt:

$$
q B R=m_{0} \gamma v
$$

Einsetzen von Gl. 35 und Auflösen nach v² liefert:

$$
v^{2}=\left(\left(\frac{m_{0}}{q B R}\right)^{2}+c^{-2}\right)^{-1}
$$

Nach dem Gleichsetzen mit Gl. 36 und Auflösen nach B ergibt sich der gesuchte relativistische Ausdruck für das Magnetfeld B in Abhängigkeit von der Ionenenergie E:

$$
B_{r e l}(E)=\frac{m_{0} c}{q R} \cdot \sqrt{\left(\frac{E}{E_{0}}+1\right)^{2}-1}
$$

Aus der klassischen Formel für die kinetische Energie $E=\frac{1}{2} m v^{2}$ ergibt sich mit dem Ansatz $\mathrm{qBR}=\mathrm{mv}$ die Formel für das Magnetfeld $\mathrm{B}_{\text {klass }}$ im nichtrelativistischen Fall:

$$
B_{\text {klass }}=\frac{1}{q R} \cdot \sqrt{2 m E}
$$

In Abb. 71 ist das Magnetfeld des $90^{\circ}$-Magneten für einen Protonen- bzw. ${ }^{15} \mathrm{~N}-\mathrm{Strahl}$ als Funktion der Ionenenergie dargestellt. Die durchgezogenen Linien zeigen das Ergebnis nach klassischer Rechnung und die Symbole ergeben sich nach dem relativistischen Ansatz. Im Maßstab von Abb. 71 sind auch für den Protonenstrahl keine Abweichungen vom klassischen Ergebnis zu erkennen. Bei Protonen der Energie E=1700 keV beträgt die Differenz zwischen klassischer und relativistischer Rechnung jedoch bereits $\Delta \mathrm{B}=100 \mu \mathrm{T}$. Da die verwendete Hall-Sonde eine Genauigkeit von ca. $5 \mu \mathrm{T}$ besitzt, sind die Unterschiede messbar. 


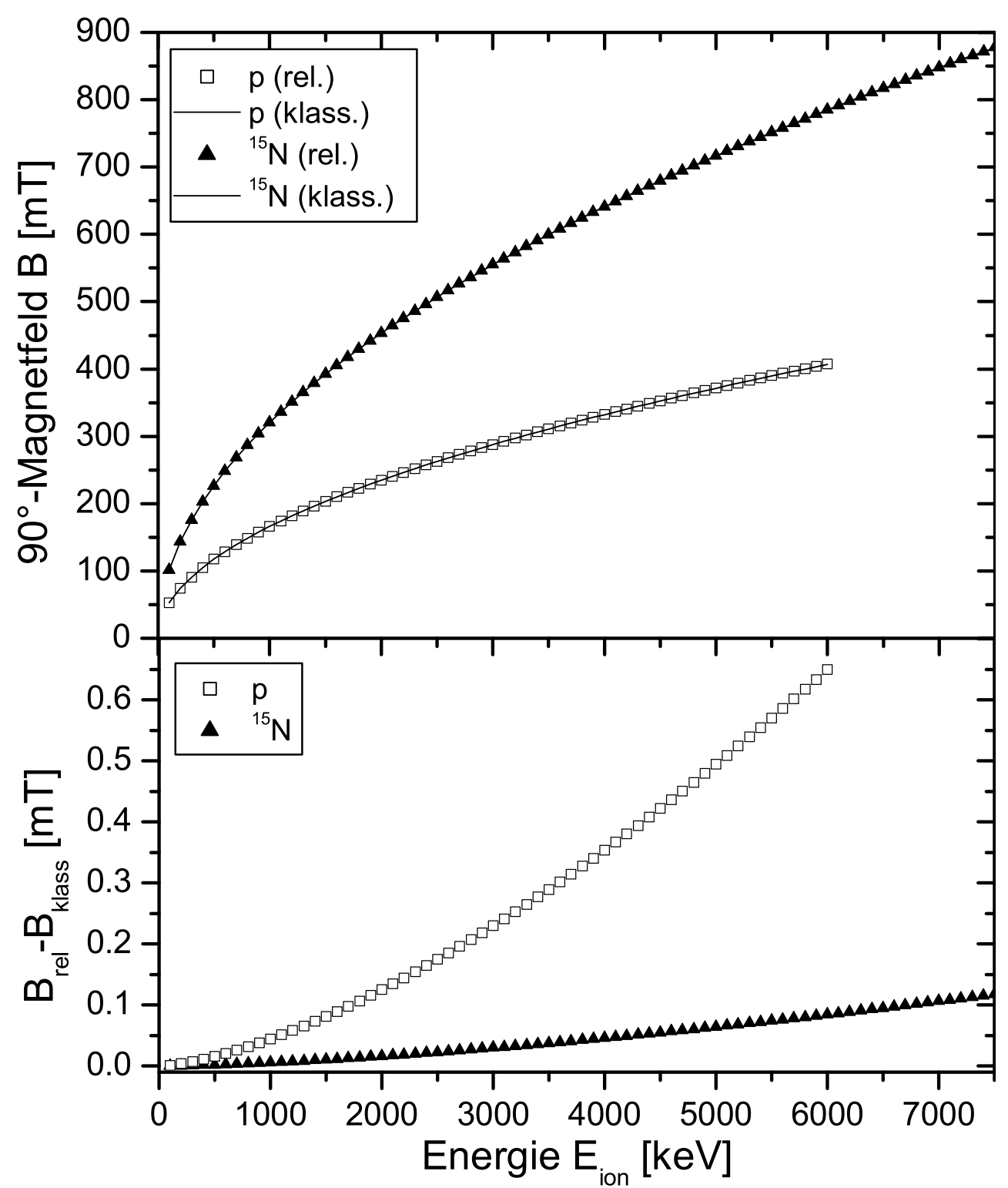

Abbildung 71: Oben: 90-Magnetfeld als Funktion der Ionenenergie für einen Protonen- bzw. ${ }^{15} \mathrm{~N}$ Strahl nach klassischer Rechnung (Linien) und relativistischer Rechnung (Symbole). Unten: Darstellung der Differenzen zwischen klassischer und relativistischer Rechnung für das Magnetfeld $B(E)$ 


\section{B Amsel-Steering-System}

Im folgenden werden die Formeln zur Berechnung des Verstärkungsfaktors $G$ hergeleitet.

\section{B.1 Amselgeometrie}

In Abb. 72 ist die Eintrittsseite des $90^{\circ}$-Magneten mit dem ersten Amsel-Deflektor schematisch dargestellt. Aus den eingezeichneten Größen können die Formeln zur Bestimmung der Abhängigkeit der Beschleunigungsspannung $V$ von der Deflektor-Spannung $U$ abgeleitet werden. Aus der Bedingung

$$
q \cdot B \cdot v=\frac{m v^{2}}{\rho}
$$

( $q$ : Ionenladung, $B$ : Magnetfeld, $v$ : Ionengeschwindigkeit, $\rho$ : Radius der Ionenbahn) folgt wegen $m v=p=\sqrt{2 m E}$ und

$$
E=\frac{q \cdot V}{K}, \text { mit } K=\frac{q}{q+\frac{m_{a}}{m_{a}+m_{b}}}
$$

( $V$ : beschleunigende Hochspannung, $m_{a}+m_{b}$ : Masse des eingeschossenen Ions (Moleküls), $m_{a}$ : Masse des Ions nach Passieren des Stripperkanals) die Gleichung für die Beschleunigungsspannung:

$$
V=\frac{(q B \rho)^{2} K}{2 m q}
$$

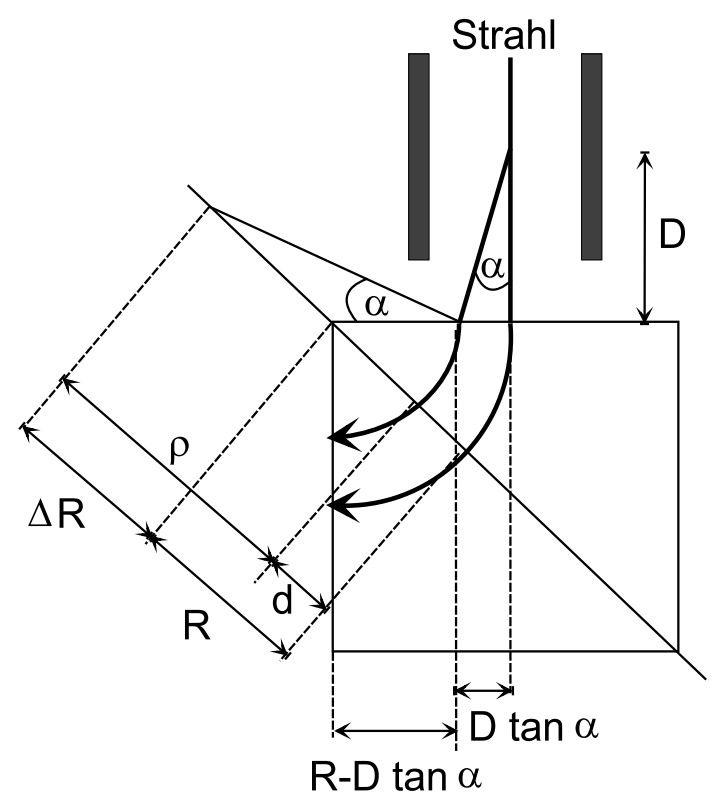

Abbildung 72: Geometrische Faktoren zur Berechnnung des Verstärkungsfaktors: Ablenkwinkel $(\alpha)$, Radius der ausgelenkten Ionenbahn $(\rho)$, Abstand der Deflektor-Mitte zum Magneten (D), Krümmungsradius des Magneten (R) 
Zur Berechnung des Ablenkwinkels $\alpha$ (vgl. Abb. 72) wird zunächst die Ablenkung des Ionenstrahls im elektrischen Feld der Deflektorplatten senkrecht zur Ionenbahn bestimmt:

$$
y\left(U, v_{x}\right)=\frac{q L^{2}}{m W} \cdot \frac{U}{v_{x}}
$$

Dabei bezeichnet $L$ die Länge der Deflektorplatten, W deren Abstand und $v_{x}$ die Ionengeschwindigkeit. In der klassischen Näherung gilt $v_{x}=\sqrt{2 E / m}$. Auf die Korrekturen für eine relativistische Rechnung wird in Abschnitt B.7 gesondert eingegangen. Zusammen mit dem Ausdruck für die Energie (Gl. 42) ergibt sich für den Ablenkwinkel $\alpha$ :

$$
\tan \alpha=\frac{y\left(U, v_{x}\right)}{L}=\frac{K L}{2 W} \cdot \frac{U}{V}
$$

Zur Berechnung des Radius $\rho$ des ausgelenkten Ionenstrahls wird zunächst der Sinussatz angewandt:

$$
\frac{\sin (180-\alpha-\varphi)}{\sin \varphi}=\frac{\rho}{(R-D \cdot \tan \alpha)}
$$

Aufgrund der Periodizität der Sinusfunktion lässt sich die linke Seite der Gleichung unter Zuhilfenahme der Additionstheoreme folgendermaßen umformen:

$$
\frac{\sin (\alpha+\varphi)}{\sin \varphi}=\frac{\sin \alpha}{\tan \varphi}+\cos \alpha
$$

Da die Strahlablenkung im Magneten $90^{\circ}$ beträgt, gilt für den Winkel $\varphi=180^{\circ}-135^{\circ}-\alpha=45^{\circ}-\alpha$. Aufgrund der Additionstheoreme für den Tangens gilt:

$$
\tan \left(45^{\circ}-\alpha\right)=\frac{\tan 45^{\circ}-\tan \alpha}{1+\tan 45^{\circ} \cdot \tan \alpha}=\frac{\cos \alpha-\sin \alpha}{\cos \alpha+\sin \alpha}
$$

Damit lässt sich Gl. 46 umformen zu

$$
\begin{aligned}
\rho & =(R-D \cdot \tan \alpha) \cdot\left(\sin \alpha \cdot \frac{\cos \alpha+\sin \alpha}{\cos \alpha-\sin \alpha}+\cos \alpha\right) \\
& =\frac{R-D \cdot \tan \alpha}{\cos \alpha-\sin \alpha}
\end{aligned}
$$

Mit den Gleichungen 43, 45 und 48, sind alle Größen zur Berechnung des Amsel-Verstärkungsfaktors G gegeben, die im nächsten Abschnitt durchgeführt wird.

\section{B.2 Exakte Berechnung des Verstärkungsfaktors G(E)}

Zur Bestimmung der Änderung der Beschleunigungsspannung V durch das Anlegen einer Deflektorspannung U wird folgender Ansatz gewählt:

$$
\frac{d V}{d U}=\frac{d V}{d \rho} \cdot \frac{d \rho}{d \alpha} \cdot \frac{d \alpha}{d U}
$$

Mit Gleichung 43 aus Abschnitt B.1 gilt:

$$
\frac{d V}{d \rho}=\frac{2 V}{\rho}
$$


Unter zweifacher Anwendung von Gleichung 48 erhält man die Ableitung

$$
\begin{aligned}
\frac{d \rho}{d \alpha} & =\frac{\frac{-D}{\cos \alpha}}{\cos \alpha-\sin \alpha}+\rho \cdot \frac{\tan \alpha+1}{1-\tan \alpha} \\
& =-\frac{D}{\cos ^{2} \alpha} \cdot \frac{\rho}{R-D \cdot \tan \alpha}+\rho \cdot \frac{\tan \alpha+1}{1-\tan \alpha}
\end{aligned}
$$

Nach dem Umstellen von Gl. 45

$$
\alpha=\arctan \left(\frac{L K}{2 W} \frac{U}{V}\right)
$$

erhält man das Differential

$$
\frac{d \alpha}{d U}=\frac{\frac{L K}{2 W V}}{1+\left(\frac{L K U}{2 W V}\right)^{2}}=\frac{\frac{L K}{2 W V}}{1+\tan ^{2} \alpha}
$$

Daraus ergibt sich durch Einsetzen der Gleichungen 50, 51 und 53 in Gleichung 49 die gesuchte Gleichung für den Amsel-Verstärkungsfaktor G:

$$
\begin{aligned}
G & =\frac{d V}{d U} \\
& =\frac{L K}{W}\left(\frac{\tan \alpha+1}{(1-\tan \alpha)\left(1+\tan ^{2} \alpha\right)}-\frac{D}{R-D \cdot \tan \alpha}\right)
\end{aligned}
$$

Durch die Abhängigkeit des Verstärkungsfaktors G vom Ablenkwinkel $\alpha$ ist $G=G(\alpha)=$ $G(E)$ nur in nullter Näherung konstant. Die Näherungsrechnung für die Energieabhängigkeit von $\mathrm{G}$ wird im nächsten Abschnitt durchgeführt.

\section{B.3 Näherung für den Verstärkungsfaktor G(E)}

Der Verstärkungsfaktor G(E) ist nach Gl. 54 eine Funktion des Winkels $\alpha$ und dieser hängt wiederum nach Gl. 52 sowohl von der Beschleunigungsspannung, d.h. von der Energie des Ionenstrahls, als auch von der angelegten Deflektorspannung U ab. Somit kann der genaue Wert von $\mathrm{G}(\mathrm{E})$ nur numerisch (vgl. Abschnitt B.4) oder aber durch eine geeignete Approximation bestimmt werden.

Zunächst wird in Gl. $54 \tan (\alpha)$ durch Gl. 45 ersetzt. Dann lässt sich G(E) durch eine Taylor-Entwicklung um den Punkt $U=0$, d.h. $\tan \alpha=0$, nähern. Diese Näherung ist sinnvoll, da beim Betrieb der Amsel-Deflektoren stets zuerst eine Basisenergie $E_{\text {base }}$ durch das feststehende Magnetfeld des $90^{\circ}$-Magneten vorgewählt wird. Das Anlegen der Deflektorspannung führt dann jeweils nur zu kleinen Änderungen der Beschleunigungsspannung um den voreingestellten Wert $V_{0}$.

$$
G(E)=G(V)=G_{0}+G_{1}\left(V_{0}, U\right)
$$

In nullter Näherung ergibt die Taylor-Entwicklung den konstanten Wert

$$
G_{0}=\frac{L K}{W} \cdot\left(1-\frac{D}{R}\right)
$$


Nach Abb. 73 steigt jedoch der Verstärkungsfaktor G mit zunehmender Ionenenergie E deutlich an, so dass die nullte Näherung zur Bestimmung von G nicht ausreicht. Die vielzitierte Eigenschaft der Amsel-Deflektoren der linearen Beziehung zwischen Deflektorspannung und Beschleunigungsspannung ist folglich für das gezeigte Beispiel eines Protonenstrahls mit Energien von weniger als $2 \mathrm{MeV}$ nur schlecht erfüllt. Zur Beseitigung der zum Teil großen Abweichungen vom linearen Verhalten (s. Abschnitt B.4) muss die Näherung erster Ordnung berücksichtigt werden. Für den nichtlinearen Anteil des Verstärkungsfaktors gilt

$$
G_{1}\left(V_{0}, U\right)=\frac{(L K)^{2}}{2 W^{2}} \cdot\left(2-\frac{D^{2}}{R^{2}}\right) \cdot \frac{U}{V_{0}}
$$

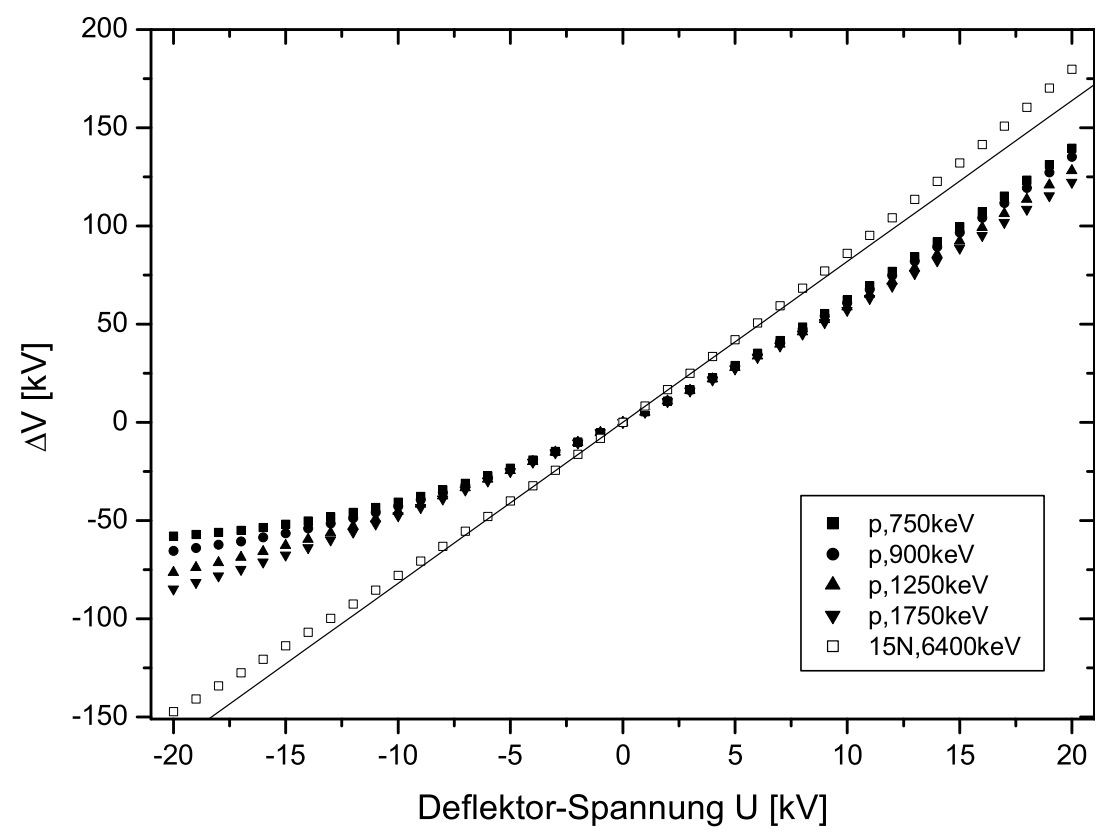

Abbildung 73: Ergebnisse der Simulation für die Änderung des Potenzials $V$ durch Anlegen der Deflektor-Spannung U. Zur Verdeutlichung der Nichtlinearität bei ${ }^{15} \mathrm{~N}$-Ionen mit $6.4 \mathrm{MeV}$ wurde zusätzlich die erwartete Änderung des Potenzials bei konstantem Verstärkungsfaktor $G$ eingezeichnet (durchgezogene Linie).

\section{B.4 Numerische Bestimmung von $G(E)$}

Der tatsächliche Gang des Verstärkungsfaktors G mit der Energie lässt sich auf Grund der Tatsache, dass $\mathrm{G}$ eine Funktion des Ablenkwinkels $\alpha$ ist und dieser wiederum nach Gl. 52 von der Beschleunigungsspannung abhängt, nur numerisch durch eine Simulationsrechnung bestimmen. Dies wurde für die Protonenenergien $750 \mathrm{keV}, 900 \mathrm{keV}, 1250 \mathrm{keV}$ und $1750 \mathrm{keV}$ durchgeführt. Diese Energien wurden als Basisenergien für die Messung von ${ }^{15} \mathrm{~N}-,{ }^{27} \mathrm{Al}-$ und ${ }^{13} \mathrm{C}$-Tiefenprofilen benutzt. Abbildung 73 zeigt neben den Simulationen für den Protonenstrahl auch das Ergebnis einer Simulation für einen $6.4 \mathrm{MeV}{ }^{15} \mathrm{~N}-\mathrm{Strahl}$, der in dieser Arbeit zur Bestimmung von Wasserstoff-Tiefenprofilen verwendet wurde. 
Wie in Abb. 73 ersichtlich, ist die Forderung der Linearität zwischen der DeflektorSpannung U und der sich daraus ergebenden Änderung der Beschleunigungs-Spannung $\Delta V$ für die hier untersuchten Protonenstrahlen nicht erfüllt. Auch die Simulation für die ${ }^{15} \mathrm{~N}$-Ionen bei $6.4 \mathrm{MeV}$ zeigt bei hohen Deflektor-Spannungen Abweichungen vom rein linearen Verhalten.

\section{B.5 Geometrische Begrenzung der Deflektor-Spannung}

Für leichte Ionen (Protonen) und geringe Ionenenergien ist die lichte Breite des Strahlrohrs am 90-Magnet der begrenzende Faktor für die Strahlauslenkung. Nach Abb. 72 bezeichnet d die maximale Abweichung des Ionenstrahls von der Bahn der Ionen ohne Ablenkspannung an den Deflektorplatten. Unter Anwendung des Sinussatzes in Abb.72 gilt

$$
\frac{\sin \alpha}{\Delta r}=\frac{\sin 135^{\circ}}{\rho}
$$

und damit folgt für $d=\Delta r+R-\rho$

$$
d=\rho(\sqrt{2} \sin \alpha-1)+R
$$

Werden die aus der Simulation (Abb. 73) bekannten Werte für die Beschleunigungsspannung V verwendet, so kann die zugehörige tatsächliche Strahlauslenkung d zu jeder Deflektor-Spannung U bestimmt werden. Dies ist in Abbildung 74 dargestellt. Nur für

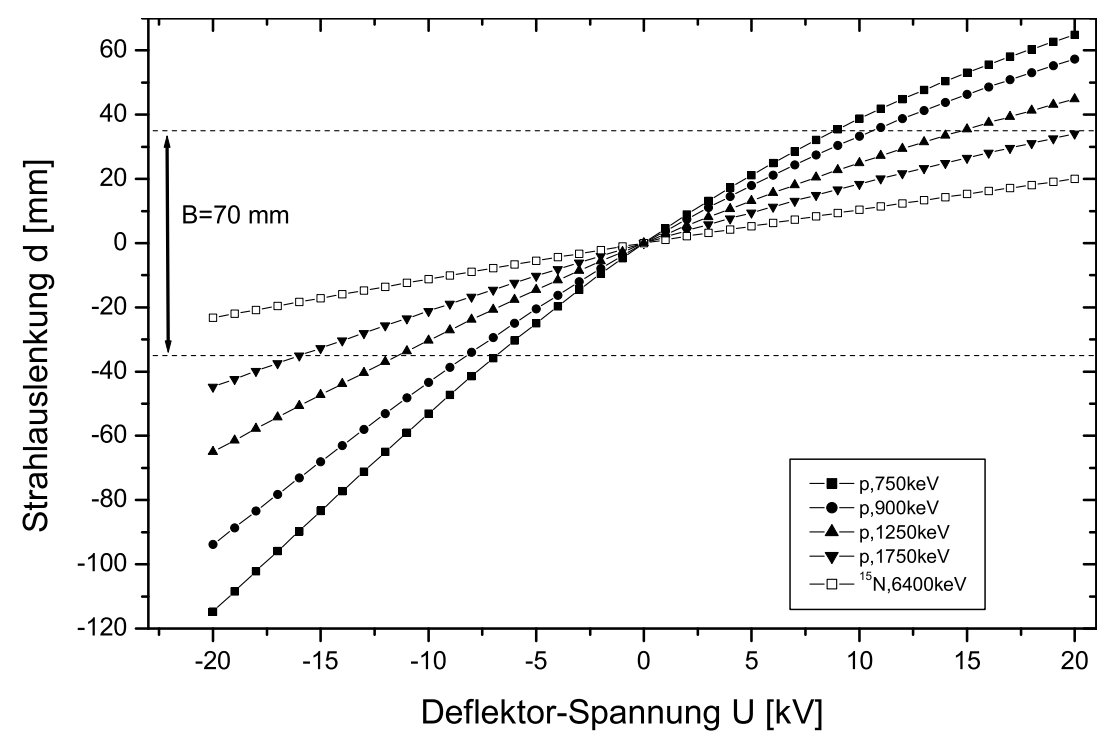

Abbildung 74: Strahlauslenkung d als Funktion der Deflektor-Spannung U. Zusätzlich ist die lichte Breite der Magnetkammer $\left(B_{M K}=70 \mathrm{~mm}\right)$ gestrichelt eingezeichnet.

die ${ }^{15} \mathrm{~N}$-Ionen bei 6.4 MeV liegt die maximale Strahlauslenkung im gesamten Spannungsbereich der Deflektoren innerhalb der geometrischen Begrenzung durch die Breite der Magnetkammer. Die Begrenzungen im Energiebereich, die sich daraus für die Deflektoren ergeben, sind in Tabelle 13 zusammengefasst. 


\begin{tabular}{l|l|l|l|l|l} 
Ion & $\mathrm{E}_{\text {base }}[\mathrm{keV}]$ & $\mathrm{U}_{\min }[\mathrm{kV}]$ & $\mathrm{U}_{\max }[\mathrm{kV}]$ & $\Delta \mathrm{V}[\mathrm{kV}]$ & $\Delta \mathrm{E}[\mathrm{keV}]$ \\
\hline \hline${ }^{1} \mathrm{H}$ & 750 & -6.8 & 8.8 & 86 & 172 \\
\hline${ }^{1} \mathrm{H}$ & 900 & -8.2 & 10.6 & 97 & 184 \\
\hline${ }^{1} \mathrm{H}$ & 1250 & -11.4 & 14.7 & 141 & 282 \\
\hline${ }^{1} \mathrm{H}$ & 1750 & -16 & 20 & 193 & 386 \\
\hline${ }^{15} \mathrm{~N}$ & 6400 & -20 & 20 & 327 & 836 \\
\hline
\end{tabular}

Tabelle 13: Eigenschaften der Amsel-Deflektoren für Protonen und ${ }^{15} \mathrm{~N}$-Strahl. E $E_{\text {base }}$ bezeichnet die mit dem $90^{\circ}$-Magneten voreingestellte Strahlenergie, $U_{\min }$ und $U_{\max }$ sind die min./max. zugängliche Deflektorspannung und $\Delta E$ bezeichnet den maximal möglichen Energiehub der AmselDeflektoren

\section{B.6 Korrektur zur Nichtlinearität von G(E)}

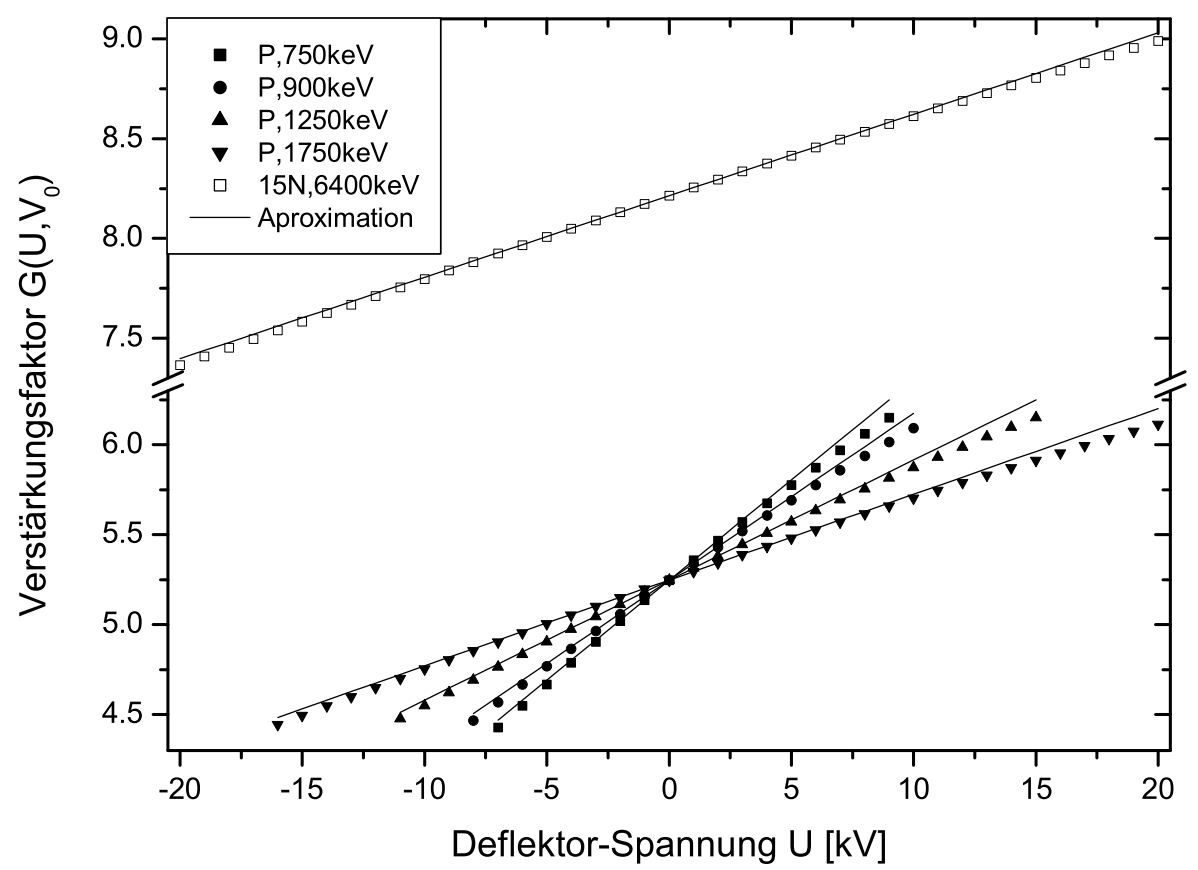

Abbildung 75: Vergleich von simuliertem Verstärkungsfaktor (Symbole) mit der Näherung 1. Ordnung nach Gl.55 (durchgezogene Linien). Zur besseren Übersicht ist die Ordinate unterbrochen dargestellt.

Abbildung 75 vergleicht die Werte für den Verstärkungsfaktor gemäß der Simulation mit den Werten, die sich aus der Näherung nach Gleichung 55 ergeben. Dabei wurde nur der Bereich der Deflektor-Spannung dargestellt, der innerhalb der in Abschnitt B.5 beschriebenen Grenzen liegt. Der nichtlineare Anteil $\mathrm{G}_{1}$ des Verstärkungsfaktors ist nach Gl. 57 antiproportional zur Beschleunigungsspannung $\mathrm{V}$ und kann für eine gegebene Ionensorte ausgedrückt werden durch:

$$
G_{1}\left(U, V_{0}\right)=\frac{(L K)^{2}}{2 W^{2}} \cdot\left(2-\frac{D^{2}}{R^{2}}\right) \cdot \frac{U}{V_{0}}=\frac{G^{*}(q, m)}{V_{0}} \cdot U
$$

Dabei bezeichnet q den Ladungszustand der Ionen nach der Umladung im Strippergas und $\mathrm{m}$ die Ionenmasse, die nach Gleichung 42 beide in den Faktor K eingehen. Damit ist 
$G^{*}$ für gegebene Ionensorte und Ladungszustand festgelegt und für die hier untersuchten Ionenstrahlen gilt:

$$
G^{*}(p)=41.7214 \text { und } G^{*}\left({ }^{15} N\right)=102.2136
$$

Für die Güte der in Abb. 75 als durchgezogene Linien dargestellten Näherungen spricht der Wert von $\chi^{2}<0.005$ bei allen fünf gezeigten Approximationen.

\section{B.7 Relativistische Berechnung von $G(E)$}

Da nach den vorigen Abschnitten Korrekturen des Verstärkungsfaktors G besonders bei leichten Ionen nötig werden, stellt sich die Frage, ob auch relativistische Effekte bei der Berechnung des Verstärkungsfaktors beachtet werden müssen. Wird die relativistische Ruheenergie mit $E_{0}=m_{0} c^{2}$ bezeichnet, so ergeben sich unter Ausnutzung der Beziehung $E=E_{k i n}=m_{0} c^{2}(\gamma-1)$ die folgenden Gleichungen für den relativistischen Faktor

$$
\gamma=\frac{E}{E_{0}}+1
$$

und die Ionengeschwindigkeit $\mathrm{v}_{\text {ion }}$

$$
v_{i o n}=\sqrt{c^{2}-\left(\frac{E_{0}}{E+E_{0}}\right)^{2}}
$$

Zur Bestimmung des Verstärkungsfaktors $G=\frac{d V}{d U}=\frac{d V}{d \rho} \frac{d \rho}{d \alpha} \frac{d \alpha}{d U}$ müssen die Differentialquotienten $\frac{d \alpha}{d U}$ und $\frac{d V}{d \rho}$ neu berechnet werden. $\frac{d \rho}{d \alpha}$ folgt aus rein geometrischen Betrachtungen und bleibt daher unverändert.

Unter Berücksichtigung der relativistischen Massenzunahme $m_{r e l}=\gamma m_{0}$ muss Gl. 41 abgeändert werden zu:

$$
q \cdot B \cdot v=\frac{m_{r e l} v^{2}}{\rho}
$$

Mit dem Einsetzen des relativistischen Impulses $p_{\text {rel }}$ und Gl. 62 folgt:

$$
q B \rho=p_{\text {rel }}=\gamma m_{0} v=\sqrt{\gamma^{2} m_{0}^{2} c^{2}-m_{0} c^{2}}
$$

Auflösen nach $\gamma$ ergibt die Form

$$
\gamma=\sqrt{\frac{1}{m_{0} E_{0}}\left((q B \rho)^{2}+m_{0} E_{0}\right)}
$$

die sich unter Ausnutzung von Gl. 61 und $V=\frac{K}{q} E$ umformen lässt zu:

$$
V_{\text {rel }}=\sqrt{(K B \rho c)^{2}+\frac{K^{2}}{q^{2}} E_{0}^{2}}-\frac{K}{q} E_{0}
$$


Die Ableitung nach dem Bahnradius $\rho$ lässt sich damit als Produkt aus dem Ergebnis nach klassischer Rechnung und einem relativistischen Faktor $R_{\text {rel }}$ schreiben:

$$
\begin{aligned}
\left(\frac{d V}{d \rho}\right)_{r e l} & =\frac{(K B c \rho)^{2}}{2 V^{2}+\frac{2 K V}{q} E_{0}} \cdot \frac{2 V}{\rho} \\
& =\left(\frac{d V}{d \rho}\right)_{k l a s s .} \cdot \underbrace{\left(\frac{1}{2+2 \frac{E_{0}}{E}}+\frac{1}{1+\frac{E}{E_{0}}}\right)}_{=: R_{\text {rel }}(E)}
\end{aligned}
$$

Zur Berechnung von $\frac{d \alpha}{d U}$ muss bei der Herleitung von G1. 52 die relativistische Massenzunahme berücksichtigt werden:

$$
\tan \alpha=\frac{q L}{\gamma m_{0} W} \cdot \frac{U}{v_{r e l}}
$$

Dabei bezeichnet $v_{r e l}$ die Ionengeschwindigkeit. Mit den Bezeichnungen $E=\frac{q}{K} V$ und $E_{0}=m_{0} c^{2}$ lässt sich dies umformen zu:

$$
\alpha=\arctan \left(\left(\frac{L}{2 W} K \frac{U}{V}\right) \cdot\left(\frac{2 E}{E_{0}} \frac{c^{2}}{\gamma^{2} v_{r e l}^{2}}\right)\right)
$$

Damit ergibt sich für den Differentialquotienten

$$
\left(\frac{d \alpha}{d U}\right)_{r e l}=\frac{\frac{L K}{2 W V}}{1+\tan ^{2} \alpha} \cdot\left(\frac{2 E}{E_{0}} \frac{c^{2}}{\gamma^{2} v_{r e l}^{2}}\right)
$$

Werden nun die Gl.61 und G1. 62 eingesetzt so folgt

$$
\left(\frac{d \alpha}{d U}\right)_{\text {rel }}=\left(\frac{d \alpha}{d U}\right)_{\text {klass }} \cdot \underbrace{\left(\frac{2 E}{E+E_{0}}+\frac{2}{\left(\frac{E}{E_{0}}\right)^{2}+3 \frac{E}{E_{0}}+2}\right)}_{=: P_{\text {rel }}(E)}
$$

Damit ergibt sich für den Verstärkungsfaktor $G=\frac{d V}{d U}$ unter Berücksichtigung relativistischer Effekte folgende Gleichung:

$$
\begin{aligned}
\left(\frac{d V}{d U}\right)_{r e l} & =\frac{d V}{d \rho} \cdot \frac{d \rho}{d \alpha} \cdot \frac{d \alpha}{d U} \\
& =\left(\frac{d V}{d U}\right)_{\text {klass }} \cdot R_{r e l}(E) P_{r e l}(E)
\end{aligned}
$$

Damit sind alle relativistischen Korrekturen in dem Faktor $R_{r e l} \cdot P_{r e l}$ zusammengefasst, der im folgenden näher betrachtet wird. Mit der Ersetzung $X=\frac{E}{E_{0}}$ folgt

$$
\begin{aligned}
R_{r e l}(E) \cdot P_{r e l}(E) & =\left(\frac{2}{1+\frac{1}{X}}+\frac{2}{X^{2}+3 X+2}\right)\left(\frac{1}{2+\frac{2}{X}}+\frac{1}{1+X}\right) \\
& =\frac{X^{2}}{(X+1)^{2}}+\frac{2 X}{(X+1)^{2}}+\frac{X+2}{X^{3}+4 X^{2}+5 X+2} \\
& =\frac{X^{2}}{(X+1)^{2}}+\frac{2 X}{(X+1)^{2}}+\frac{1}{(X+1)^{2}} \\
& =1
\end{aligned}
$$


Dieses Ergebnis zeigt, dass sich die relativistischen Effekte, die beim Betrieb mit leichten Ionen hoher Energie auftreten, beim Verstärkungsfaktor gerade kompensieren. Das Ergebnis der klassischen Rechnung stimmt also mit dem der relativistischen Rechnung genau überein. Folglich müssen keine relativistischen Korrekturen beim Verstärkungsfaktor G vorgenommen werden.

\section{Nachweisempfindlichkeit der Low-Level NRA}

Wie in Abschnitt 2.2 beschrieben, muss die gemessene Gamma-Ausbeute $Y_{M}$ zur Bestimmung der Konzentrationsprofile auf die deponierte Ladung $Q=I \cdot t$ normiert werden, d.h. für die normierte Gamma-Ausbeute $Y_{\text {norm }}$ gilt:

$$
Y_{\text {norm }}=\frac{Y_{R}}{I \cdot t}=K \cdot c_{\text {min }}
$$

Zur Berechnung der Nachweisempfindlichkeit der Low-Level NRA soll zunächst der relative Fehler $\frac{\Delta Y_{R}}{Y_{R}}$ der Kernreaktionsereignisse $Y_{R}$ in Abhängigkeit von der gemessenen Signalrate $\dot{Y}_{M}$ und der Untergrundrate $\dot{Y}_{U G}$ bestimmt werden.

Die tatsächlichen Kernreaktionsereignisse sind eine Funktion von Strahlstrom I und Messdauer $t$ und es gilt:

$$
Y_{R}(I, t)=Y_{M}(I, t)-Y_{U G}(t)
$$

Mit der Gaußschen Fehlerfortpflanzung folgt für den Fehler von $Y_{R}$ unter der Voraussetzung, dass der Fehler der Strahlstrommessung zu vernachlässigen ist

$$
\begin{aligned}
\Delta Y & =\sqrt{\left(\frac{d Y_{R}}{d Y_{M}} \frac{d Y_{M}}{d t} \Delta t\right)^{2}+\left(\frac{d Y_{R}}{d Y_{U G}} \frac{d Y_{U G}}{d t} \Delta t\right)^{2}} \\
& =(\Delta t) \sqrt{\dot{Y}_{M}} \cdot \sqrt{\dot{Y}_{M}+2 \dot{Y}_{U G}+2 \frac{\dot{Y}_{U G}^{2}}{\dot{Y}_{M}}}
\end{aligned}
$$

Mit $\Delta t=\frac{t}{\sqrt{Y_{R}}}$ und der zeitlichen Linearität von $Y_{R}$ (Gl. 74 ) ergibt sich für den gesuchten relativen Fehler der Zusammenhang:

$$
\frac{\Delta Y_{R}}{Y_{R}}=\frac{\sqrt{\dot{Y}_{R}+2 \dot{Y}_{U G}+2 \frac{\dot{Y}_{U G}^{2}}{\dot{Y}_{R}}}}{\dot{Y}_{R} \sqrt{t}}
$$

Dieser Ausdruck soll nun nach der Signalrate $\dot{Y}_{R}$ aufgelöst werden. Umstellen von Gl.77 ergibt

$$
\dot{Y}_{R}^{3}-\left(\left(\frac{\Delta Y_{R}}{Y}\right)^{2} t\right)^{-1} \cdot\left(\dot{Y}_{R}+\dot{Y}_{U G}\right)^{2}-\left(\left(\frac{\Delta Y_{R}}{Y}\right)^{2} t\right)^{-1} \dot{Y}_{U G}^{2}=0
$$

Es soll die minimal nachweisbare Konzentration $c_{\min }$ berechnet werden, die mit sehr kleinen Signalraten $\dot{Y}_{R}$ verbunden ist. Für diesen Extremfall gilt $\dot{Y}_{R} \ll \dot{Y}_{U G}$ und in Gl.78 kann die Näherung $\dot{Y}_{R}+\dot{Y}_{U G} \approx \dot{Y}_{U G}$ eingesetzt werden. Damit folgt

$$
\dot{Y}_{R}^{3}-\frac{2}{\left(\frac{\Delta Y_{R}}{Y}\right)^{2} t} \cdot \dot{Y}_{U G}^{2}=0
$$


Somit folgt für die Signalrate $\dot{Y}_{R}$ bei sehr kleinen Konzentrationen der nachzuweisenden Atomsorte der Ausdruck

$$
\dot{Y}_{R}\left(\dot{Y}_{U G}, \frac{\Delta Y_{R}}{Y_{R}}, t\right)=\sqrt[3]{\frac{2 \dot{Y}_{U G}^{2}}{\left(\frac{\Delta Y_{R}}{Y_{R}}\right)^{2} t}}
$$

Nun kann nach G1.74 die zu einem vorgegebenen relativen Fehler $\frac{\Delta Y_{R}}{Y_{R}}$ nachweisbare Minimalkonzentration $c_{\min }$ in Abhängigkeit von der Untergrundrate $\dot{Y}_{U G}$, der Messdauer $t$ und dem Messstrom $I$ berechnet werden

$$
c_{\text {min }}=\frac{\dot{Y}_{R}}{K \cdot I}=\frac{1}{K \cdot I} \cdot \sqrt[3]{\frac{2 \dot{Y}_{U G}^{2}}{\left(\frac{\Delta Y_{R}}{Y_{R}}\right)^{2} t}}
$$

Damit ist gezeigt, dass eine Erhöhung des Messstroms den größten Einfluss auf die Nachweisgrenze der NRA-Messung hat. Eine Erhöhung des Messstroms um eine Größenordnung senkt die Nachweisgrenze um einen Faktor 10 herab. Jedoch ist der Erhöhung des Strahlstroms durch die Ionenquelle und die folgende Ionenoptik eine natürliche Grenze gesetzt. Der in der NRA-Kammer maximal erreichbare Targetstrom liegt bei ca. $1.5 \mu \mathrm{A}$. Im Zusammenhang mit dem Low-Level Messaufbau ist natürlich der Einfluss der Untergrundrate $Y_{U G}$ von besonderem Interesse. Gemäß Gl. 81 führt eine Verminderung der Untergrundrate um einen Faktor 10 zu einer um den Faktor 4.64 verbesserten Nachweisempfindlichkeit.

Wird die Messzeit um einen Faktor 10 verlängert, so vermindert sich dadurch die Nachweisgrenze um den Faktor 2.15. 


\section{Realtime-Linux, $\mathrm{Qt}^{\circledR}{ }^{\circledR}$ und $\mathrm{KDE}$}

\section{Realtime-Linux}

Die Grundlage für das in der vorliegenden Arbeit entwickelte NRA-Messprogramm bildet das Open Source Projekt Realtime Linux (RTL). Die Idee von Realtime Linux besteht darin, dass ein Mini-Linux Kernel als eigenständiges Betriebssystem läuft, welches das standard Linux Betriebssystem als einen Task mit niedrigster Priorität ablaufen lässt. Dazu wird ein zusätzlicher Abstraction Layer (auch ,virtual machine“) zwischen der PCHardware und dem standard Linux Kernel eingefügt (s. Abb. 76). Der RTL Abstraction Layer fängt alle Hardware Interrupts ab und gibt die nicht zeitkritischen Tasks als Software Interrupts an den standard Linux-Kernel weiter. Zeitkritische Aufgaben werden dagegen von der RTL-Interrupt-Service-Routine abgearbeitet, die den Vorteil fest vorgegebener Bearbeitungszeiten bietet. Auf dem verwendeten Pentium II $300 \mathrm{MHz}-$ Rechner erreicht RTL eine sog. ,worst case interrupt latency“ von weniger als $12 \mu \mathrm{s}$ und eine ,average interrupt latency" von weniger als $5 \mu \mathrm{s}$.

Der Datentransport vom RT-Kernel zur Software-Ebene läuft dabei über FIFO-Speicher (first-in-first-out), so dass die Daten von dort zeitunkritisch weiter verarbeitet werden können.

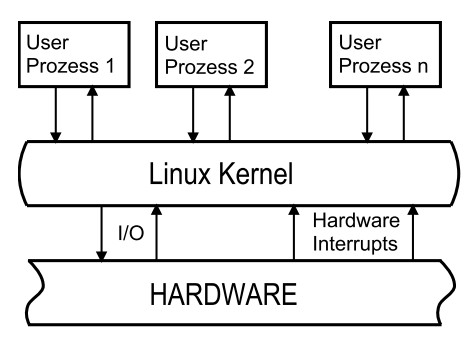

a)

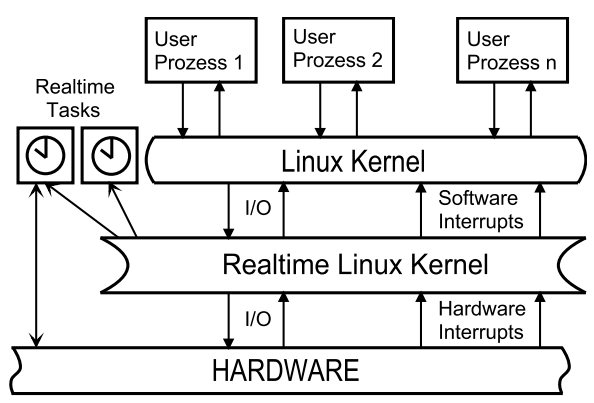

b)

Abbildung 76: Prinzip des Realtime Linux Kernels: a) Standard Linuxsystem, b) Realtime Linuxsystem: Durch den kleinen Realtime Kernel als zusätzlichem „Abstraction Layer" werden unvorhersagbare Zeitverzögerungen bei der Bearbeitung zeitkritischer Tasks umgangen

\section{$\mathrm{Qt}^{\circledR}$ und das K Desktop Environment (KDE)}

Bei $\mathrm{Qt}^{\circledR}{ }^{\circledR}$ handelt es sich um eine von der norwegischen Firma „Troll Tech“ [128] entwickelte $\mathrm{C}++$-Bibliothek, die plattformübergreifend, d.h. für verschieden Betriebsysteme, verwendet werden kann. Für eine nicht-kommerzielle Nutzung steht das Qt-Paket unter der GNU Public Licence, d.h. es darf ohne Lizenzgebühren genutzt werden, solange die Regeln der GNU Public Licence [129] beachtet werden. Die Qt-Bibliothek stellt Klassen und Methoden zur GUI-Programmierung („Graphical User Interface“) zur Verfügung, mit deren Hilfe grafische Bedienelemente (Fenster, Schaltflächen usw.) mit geringem Zeitaufwand programmiert werden können. Eine Besonderheit von Qt ist die Idee des ,SignalSlot"-Mechanismus, der es auf einfache Weise erlaubt, komplexe Abläufe im Computerprogramm abzubilden. Die mittlerweile bei Linux-Systemen sehr weit verbreitete grafische Benutzeroberfläche KDE ist vollständig unter Qt programmiert. So verwendet auch der NRA-Messrechner KDE. 


\section{E Bilder vom Umzug des MaRPel-Labors}

\section{E.1 Abbau in Heidelberg}

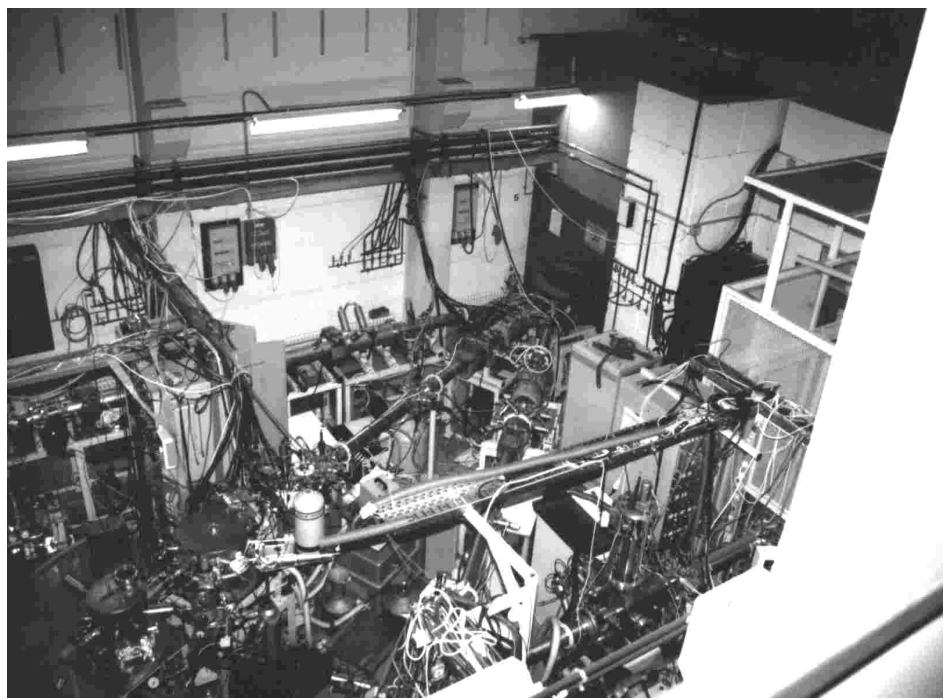

Abbildung 77: Das Halbleiter-Labor (HLL) im Kellerlabor des Bothe-Gebäudes im Max-PlanckInstitut für Kernphysik in Heidelberg vor dem Abbau. Das Bild zeigt die Experimentierhalle. Der Beschleunigerbereich ist aus Strahlenschutzgründen durch eine Wand abgetrennt (rechts im Bild)

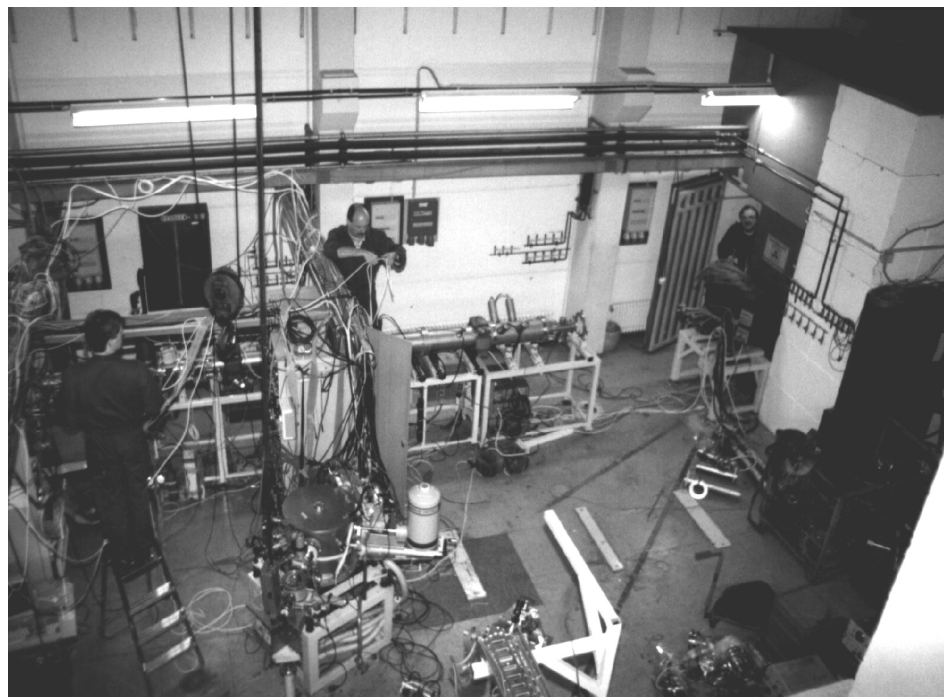

Abbildung 78: Demontage der Strahlrohre in der Experimentierhalle des HLL. Am oberen Rand ist das $0^{\circ}$-Strahlrohr zu sehen, an dessen Ende sich der Low-Level Messplatz befindet. Das $25^{\circ}$ und $45^{\circ}$-Strahlrohr sind zu diesem Zeitpunkt bereits demontiert. 


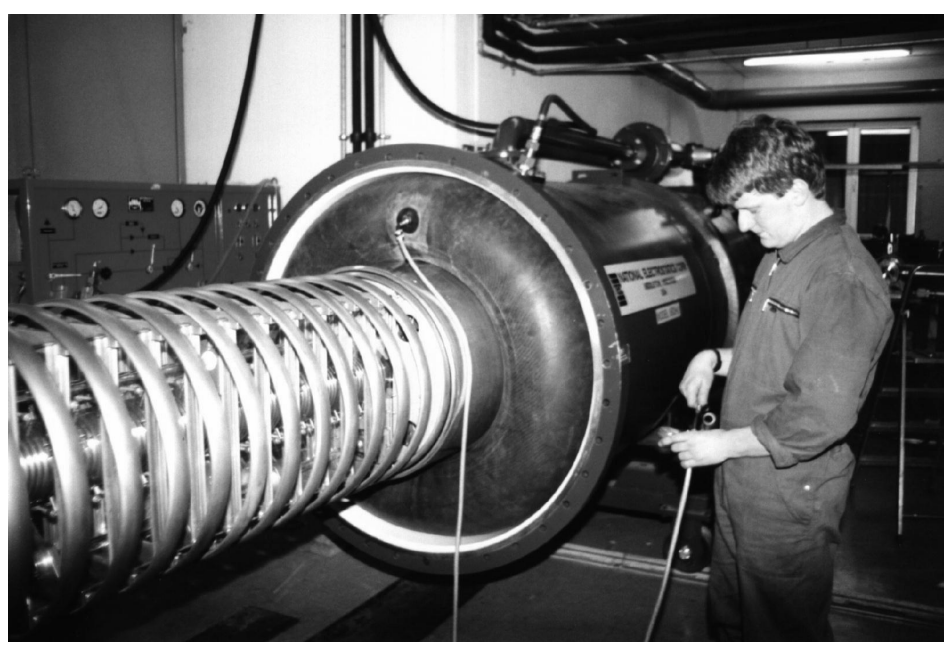

Abbildung 79: Für den Transport wurde die Beschleuniger-Säule durch einen LKW-Schlauch gesichert.

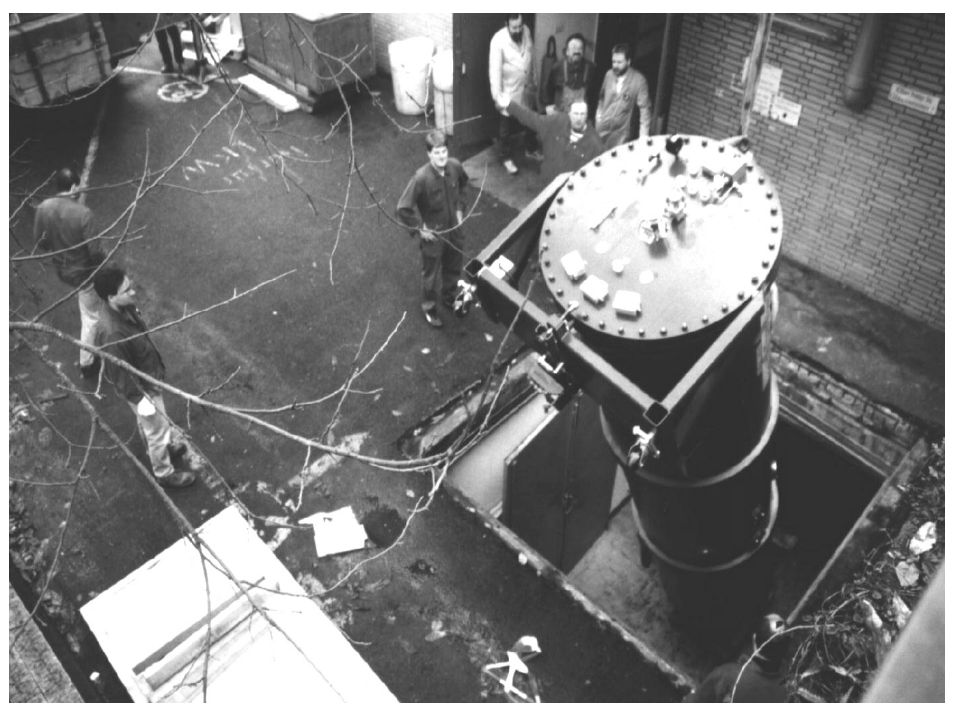

Abbildung 80: Der Beschleunigertank konnte nur über den im Bild gezeigten Zugang den Keller des Bothe-Labors verlassen. Dazu musste der Autokran zentimetergenau gesteuert werden. 


\section{E.2 Aufbau in Göttingen}

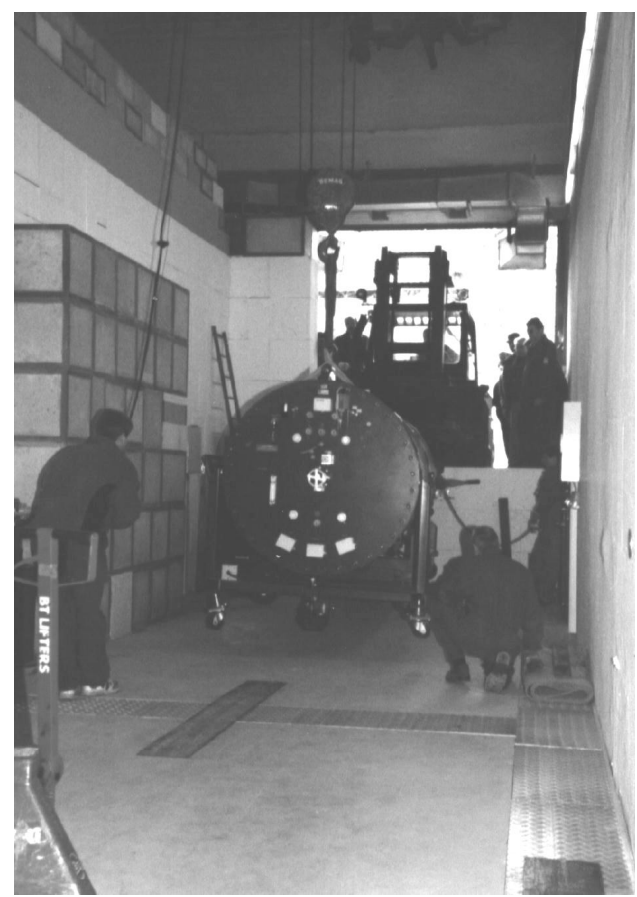

Abbildung 81: Am Schwerlast-Kran hängend wird der Beschleunigertank vom Autor in seine endgültige Position gebracht.

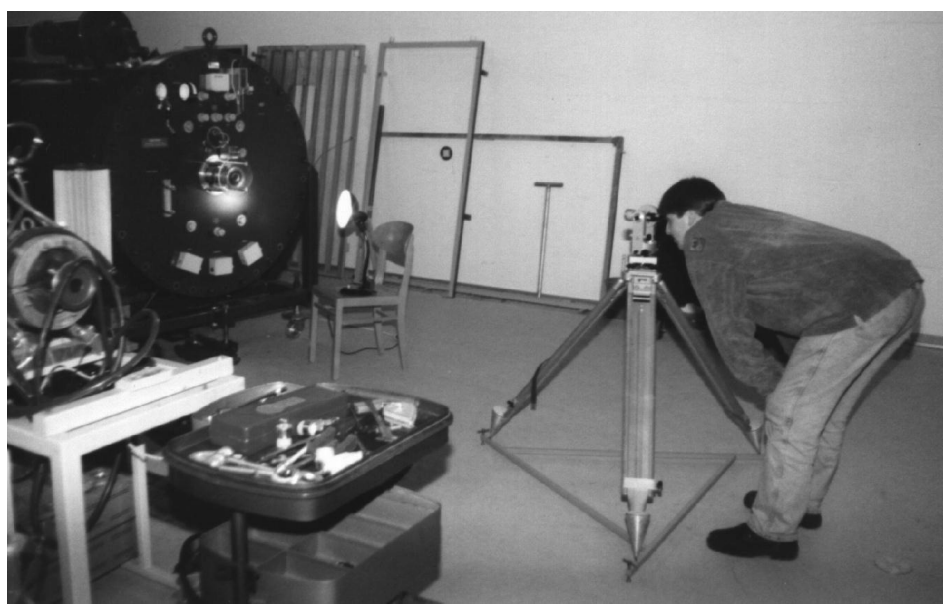

Abbildung 82: Alle Teile der Ionenoptik wurden mit Hilfe von Theodoliten, einem Justierlaser und Wandmarken ausgerichtet. 


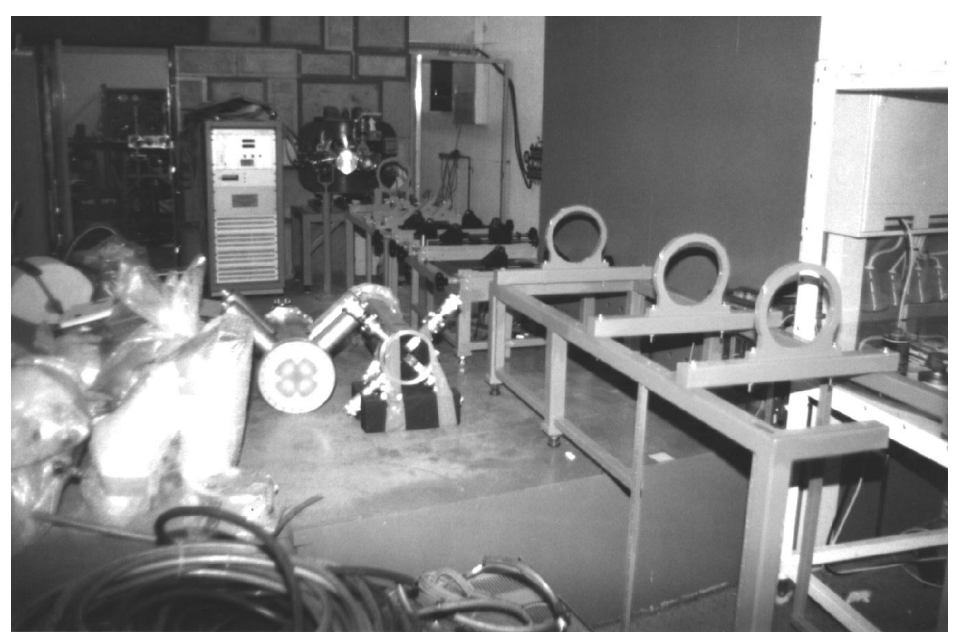

Abbildung 83: Die Strahlrohrteile des Nullgrad-Strahlrohrs, an dessen Ende später die Low-Level NRA-Messapparatur aufgebaut wurde, liegen noch verpackt im Magnetjoch des ehemaligen Zyklotrons.

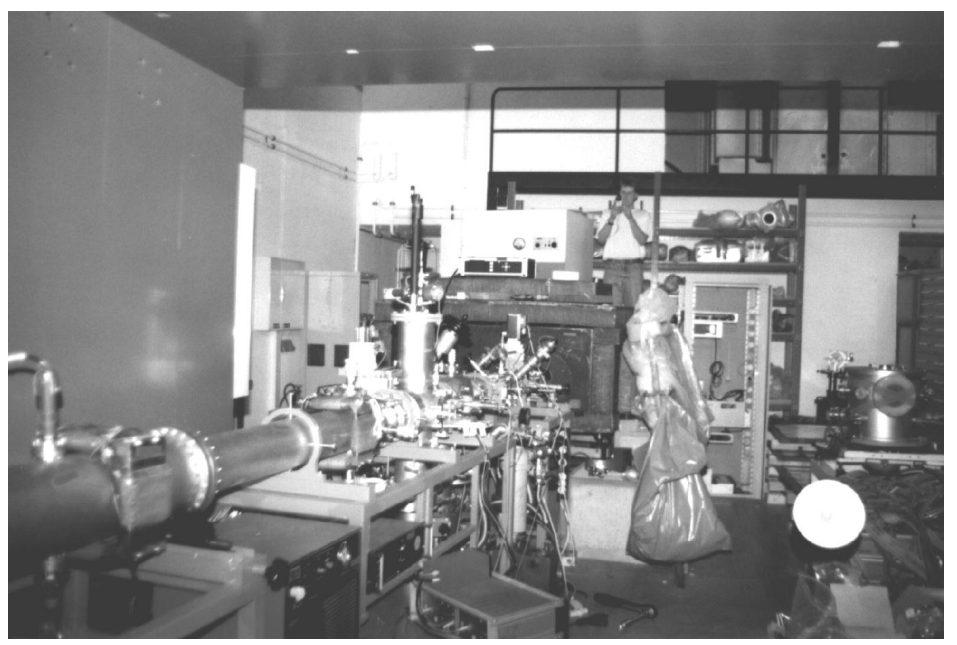

Abbildung 84: Alle Teile des Nullgrad-Strahlrohrs und die Eisenabschirmung der Low-Level Apparatur sind fertig aufgebaut und vakuumgetestet. In diesem Bild ist die Eisenabschirmung noch nicht von den Szintillator-Platten zur Antikoinzidenz-Messung umgeben. 


\section{F Phasendiagramme}

\section{F.1 Ti-H-Phasendiagramm}

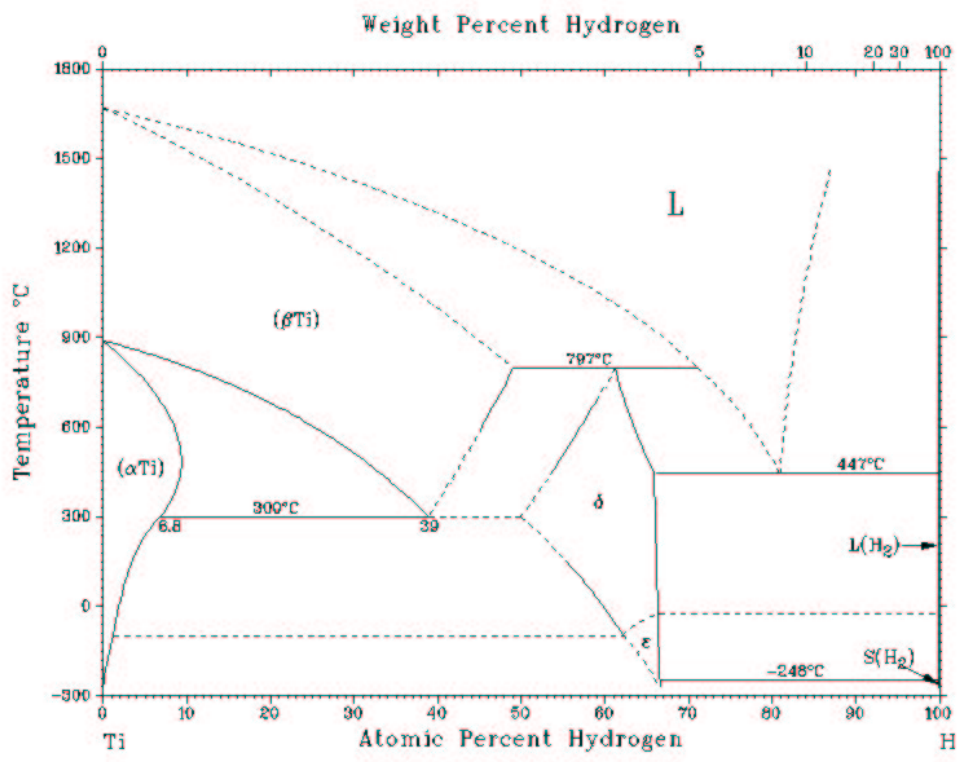

Abbildung 85: Ti-H-Phasendiagramm, aus [130]

\section{F.2 Si-H-,,Phasendiagramm“6}

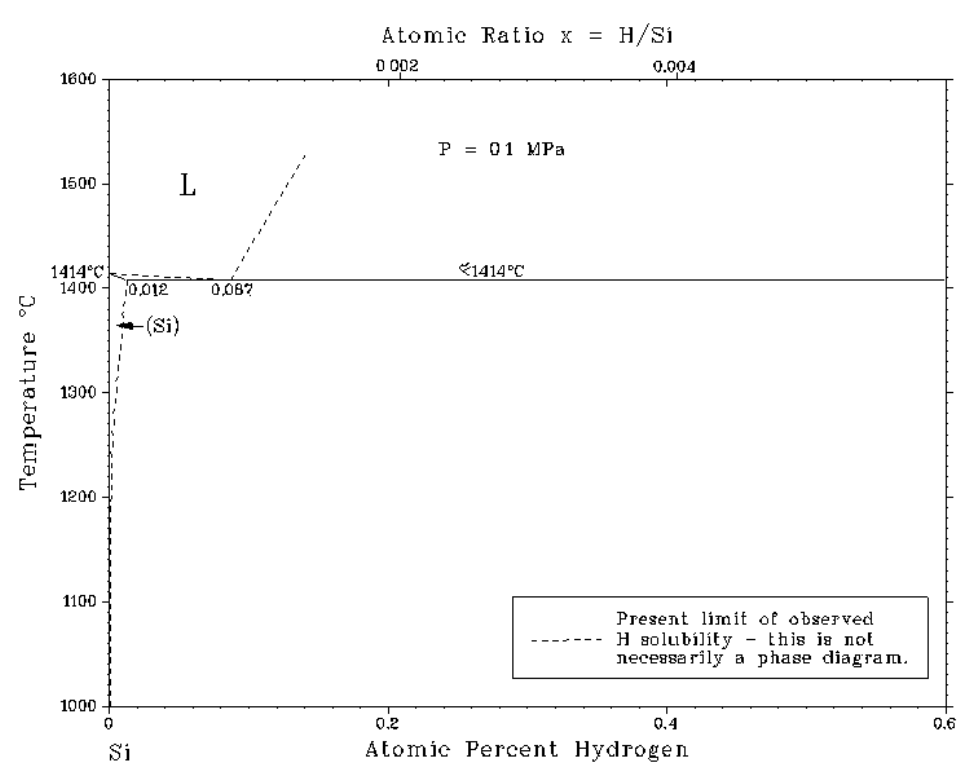

Abbildung 86: Si-H-,,Phasendiagramm“, aus [131] 


\section{Danksagung}

Ein so großes Projekt, wie der Umzug eines kompletten Beschleunigerlabors, kann nicht ohne die Hilfe sämtlicher wissenschaftlichen und technischen Mitarbeiter vonstatten gehen.

An erster Stelle möchte ich mich bei Hr. Prof. Dr. Lieb bedanken, für die Idee und das Durchsetzungsvermögen, mit dem es gelang, den Zuschlag für die Schenkung des MaRPelLabors zu bekommen.

Bei Dr. M. Uhrmacher möchte ich mich für seine stete Diskussionsbereitschaft und seine herzliche Art bedanken. Ohne die vielen Aufmunterungen, wenn nach n Reparaturen das (n+1)-te Elektronikteil ausfiel, würde MaRPel wahrscheinlich auch heute noch keinen Ionenstrahl produzieren. Auch für viele abendliche Überstunden bei den MaRPelStrahlzeiten muss ich mich bei ihm, vor allem aber auch bei seiner Frau bedanken.

PD Dr. Peter Schaaf danke ich sehr für die Einführung und Hilfe bei den Laserbestrahlungen und für viele Anregungen zu den Experimenten.

Holger Schebela gebührt großer Dank, da er durch seine Fähigkeiten (Elektrik/ Elektronik/ Feinmechanik) zum Gelingen des „Projekts Pelletron“ einen außerordentlich großen Beitrag geleistet hat. Außerdem hatten wir auch an Tagen, an denen plötzlich alles ausfiel, gemeinsam noch ausreichend Galgenhumor. Für die gute Arbeitsatmosphäre und seine hilfsbereite Art möchte ich mich ausdrücklich bedanken.

Dr. Felix Harbsmeier danke ich ganz besonders. Es war uns beiden von Anfang an klar, dass der Pelletron-Umzug eine wahre Herausforderung darstellt, die wir meistern wollten. Felix gebührt nicht nur für einen Großteil der Umzugsplanung und viele Überstunden beim Abbau in Heidelberg und Aufbau in Göttingen dank. Durch seinen Teamgeist und den ausgeprägten Sinn für Humor wurde vieles einfacher.

Ettore Carpene is greatly acknowledged for his help during the XRD-measurements, his interest in physical discussions and so many big Guiness!

I'm also indebted to Dr. Sankar Dhar, who always had time to discuss the mysteries of recrystallization. Thank you also for the nice evenings we had at your house.

Alexander Müller danke ich für seine Hilfe nicht nur bei Ionas-Strahlzeiten, besonders in der Endphase der Arbeit.

Stanislawa Gasiorek danke ich dafür, dass Sie mir ihren bravourösen „Target-Halter Nr. 1" zur Verfügung gestellt hat, denn nur mit ihm liessen sich die Si-Proben channeln.

Daniel Schwen und Jürgen Appel danke ich für den ADC-Treiber und viele gute Tipps bezüglich Realtime-Linux, Qt und $\mathrm{C}++$.

Detlef Purschke danke ich für die gute Atmosphäre bei vielen Stunden Strahlzeit am IONAS. Auch für seine Bemühungen gegen die zahlreichen Hochspannungs-Überschläge bei den letzten Messungen möchte ich ihm danken. 
Großer Dank geht auch an die Feinmechanik-Werkstatt, ohne deren ständige Mitarbeit und Hilfe der Umzug undenkbar gewesen wäre. In nahezu jeder Phase waren wir auf Vorarbeiten und Hilfe der Werkstatt angewiesen, auf die man sich stets verlassen konnte. Dabei war oft, z.B. beim Abbau des Zyklotrons, mehr die „Grobmechanik“ gefragt. Hans-Jürgen Obal möchte ich besonders für die enorme Hilfe beim Abbau in Heidelberg danken, sowie für seine ständige Hilfsbereitschaft, wenn wieder mal etwas sofort erledigt werden musste. Klaus Arndt und Christof Schmidt sei für Ihre große Hilfe bei allen Neukonstruktionen gedankt und dafür, dass Aufträge für das Pelletron stets eine hohe Priorität bekamen. Andreas König und Michael Laugsch danke für viele Gespräche über die wesentlichen Dinge im Leben und den Spaß, den wir zusammen hatten. Bei Peter Arnsberger möchte ich mich für die hervorragende Wartung der verschiedenen Vakuumpumpen bedanken.

Herwig Schultz danke ich für die Hilfe bei allen Fragen der Elektrik und des Anglerlateins.

Die Elektronik-Werkstatt hat entscheidend zur Instandsetzung und Erneuerung der zum Teil recht altertümlichen Elektronikteile des MaRPel-Labors beigetragen. Ich möchte mich besonders bei Kurt Schemmerling für seine PCI-ADC-Karte bedanken und für die Konstruktion des Zähler-Moduls. Für die entscheidenden Tests der Energiestabilisierung bedanke ich mich ganz besonders bei Michael Hillmann, Volker Ehbrecht und Reinhard Mielke.

Den „guten Geistern“ des Instituts Christa Wohlfarth, Luci Hamdi und Heike Ahrens danke ich dafür, dass sie immer da waren, wenn man Hilfe brauchte.

Stellvertretend für das Max-Planck-Institut für Kernphysik in Heidelberg möchte ich Dr. Bernd Martin und Dr. Christopher Klatt danken. Sie haben uns beim Umzug sehr schnell und tatkräftig unterstützt. Dr. Klatt hat mir eine sehr fundierte Einführung in die Steuerung des Beschleunigers gegeben, wofür ich mich bedanken möchte.

Tonja bin ich ausgesprochen dankbar, dass sie mir jederzeit eine große Stütze war und ist. Wenn mal wieder gar nichts funktionierte konnte ich mir Deiner Aufmunterung sicher sein. Vielen Dank auch für das schnelle Korrekturlesen!

Meiner Mutter bin ich zu größtem Dank verpflichtet. Ich weiß, dass die letzten beiden Jahre die schlimmsten ihres Lebens waren und dass ich oft zu Hause wichtiger gewesen wäre, als im MaRPel-Labor. Ich danke Dir dafür, das Du trotzdem alles, viel zu oft allein, gemeistert hast. 


\section{Lebenslauf}

Am 29. Mai 1972 wurde ich, Marcus Schwickert, als Sohn meiner Eltern Werner und Regina Schwickert, geb. Bednarck, in Koblenz geboren.

Von 1978 bis 1982 besuchte ich die Goethe-Grundschule Höhr-Grenzhausen. 1982 wechselte ich auf das Gymnasium im Kannenbäckerland Höhr-Grenzhausen. Dort legte ich im Juni 1991 das Abitur ab.

Von August 1991 bis Oktober 1992 leistete ich den Zivildienst in der individuellen Schwerstbehindertenbetreuung der Arbeiterwohlfahrt ab.

Mit Beginn des Wintersemesters 1992 nahm ich das Studium der Physik an der Universität Bonn auf. Im Oktober 1994 legte ich dort das Vordiplom ab. Zum Wintersemester 1995 wechselte ich von Bonn an die Universität Göttingen und setzte dort das Physikstudium fort.

Im Oktober 1996 begann ich im II. Physikalischen Institut unter Anleitung von Prof. Dr. K. P. Lieb mit meiner Diplomarbeit zum Thema „Modifikationen von a- $\mathrm{SiO}_{2}$ und $\mathrm{Ni}$ /a$\mathrm{SiO}_{2}$-Schichten durch Ar- und Xe-Implantation“. Im Mai 1998 legte ich die Diplomprüfung im Fach Physik ab.

Anschließend nahm ich die Arbeit an der vorliegenden Dissertation auf. Seit April 1996 betreue ich als wissenschaftliche Hilfskraft bzw. seit Oktober 1998 als wissenschaftlicher Mitarbeiter am II. Physikalischen Institut Studenten im Haupt- und Nebenfachpraktikum Physik. 